University of New Hampshire

University of New Hampshire Scholars' Repository

Spring 2002

\title{
Human -machine system design optimization for nondeterministic spacecraft anomaly determination/resolution
}

Kriss Quinn Hunold

University of New Hampshire, Durham

Follow this and additional works at: https://scholars.unh.edu/dissertation

\section{Recommended Citation}

Hunold, Kriss Quinn, "Human -machine system design optimization for nondeterministic spacecraft anomaly determination/resolution" (2002). Doctoral Dissertations. 68.

https://scholars.unh.edu/dissertation/68

This Dissertation is brought to you for free and open access by the Student Scholarship at University of New Hampshire Scholars' Repository. It has been accepted for inclusion in Doctoral Dissertations by an authorized administrator of University of New Hampshire Scholars' Repository. For more information, please contact Scholarly.Communication@unh.edu. 


\section{INFORMATION TO USERS}

This manuscript has been reproduced from the microfilm master. UMI films the lext directly from the original or copy submitted. Thus, some thesis and dissertation copies are in typewriter face, while others may be from any type of computer printer.

The quality of this reproduction is dependent upon the quality of the copy submitted. Broken or indistinct print, colored or poor quality illustrations and photographs, print bleedthrough, substandard margins, and improper alignment can adversely affect reproduction.

In the unlikely event that the author did not send UMI a complete manuscript and there are missing pages, these will be noted. Also, if unauthorized copyright material had to be removed, a note will indicate the deletion.

Oversize materials (e.g., maps, drawings, charts) are reproduced by sectioning the original, beginning at the upper left-hand comer and continuing from left to right in equal sections with small overtaps.

Photographs included in the original manuscript have been reproduced xerographically in this copy. Higher quality $6^{\circ} \times 9^{\circ}$ black and white photographic prints are available for any photographs or illustrations appearing in this copy for an additional charge. Contact UMI directly to order.

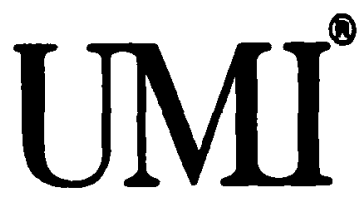




\title{
HUMAN-MACHINE SYSTEM DESIGN OPTIMIZATION FOR NON- DETERMINISTIC SPACECRAFT ANOMALY DETERMINATION/RESOLUTION
}

\author{
BY \\ KRISS QUINN HUNOLD \\ B.S., Aeronautical Engineering, Embry-Riddle Aeronautical University, 1989 \\ M.A., Space Systems Management, Webster University, 1993 \\ M.S., Statistics, University of Southern Maine, 1997

\begin{abstract}
DISSERTATION
Submitted to the University of New Hampshire

in Partial Fulfillment of

the Requirements for the Degree of
\end{abstract} \\ Doctor of Philosophy \\ In \\ Engineering: Systems Design
}

May, 2002 
UMI Number: 3045328

Copyright 2002 by

Hunold, Kriss Quinn

All rights reserved.

\section{UMI}

UMI Microform 3045328

Copyright 2002 by ProQuest Information and Learning Company.

All rights reserved. This microform edition is protected against unauthorized copying under Title 17, United States Code.

\section{ProQuest Information and Learning Company 300 North Zeeb Road \\ P.O. Box 1346 \\ Ann Arbor, MI 48106-1346}




\section{ALL RIGHTS RESERVED}

C 2002

Kriss Quinn Hunold 


\section{Ph.D. DISSERTATION}

This dissertation has been examined and approved.

\section{QheRhalmue}

Dissegtation Director, John R. LaCourse, Professor and Chair, Electrical and Computer Engineering. Unitstrsity of New Hampshire
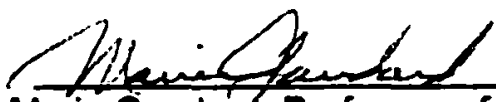

Marie Gaudard, Professor of Mathematics and Statistics, University of New Hampshire

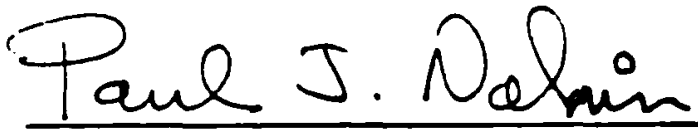

Paul Nahin, Professor of Electrical and Computer Engineering, University of New Hampshire

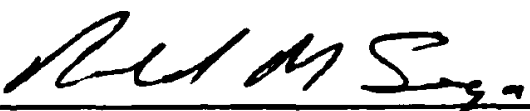

Ronald M. Sega, Director of Defense Research and Engineering; Professor of Electrical and Computer Engineering, University of Colorado Colorado Springs

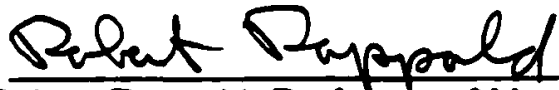

Robert Rappold, Professor of Mechanical

Engineering, University of Colorado - Colorado Springs

Date:

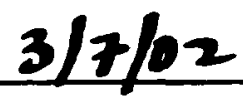




\section{DEDICATION}

This is dedicated to my family -- especially Mom (the esteemed proofreader), and my biggest supporter: my husband Rob. Thank you all for your loving support and encouragement. 


\section{ACKNOWLEDGEMENTS}

There are many people who have been selfless in contributing to this effort, and I thank all of you for your time and hard work. I owe everything to you!

- My deep appreciation goes to all of the committee members, who were exceptionally helpful and supportive in developing and validating the material. Thanks for making this process enjoyable.

- The Air Force and contractor personnel from the Center for Research Support -thank you for your support of this project. l'd like to extend thanks to Jerry Safranek. Karyn Fishburn, Steve Scholz, Mark Stafford, and especially Chad Oster.

- Much gratitude to the leadership, volunteers, and support personnel of both the $1^{\text {st }}$ Space Operations Squadron and $7^{\text {th }}$ Space Operations Squadron. Lt Col Mike Chesonis, Maj Mary McKeon, Capt Mike Marrs and SSgt Agnew were exceptionally supportive and helpful. Capt Tim Franklin and SrA Bob Kerr provided invaluable assistance during data collection. Thank you to all!

- Special thanks for the technical expertise and advice of Paul Loomis and Mitch Norder, as well as Shari Striebel-Thornton and the other TRW and AEROJET professionals at Schriever AFB.

- I'd like to acknowledge Marie Gaudard, and thank her for suffering through multiple readings. You were not only an invaluable help, but also an encouraging friend.

I would especially like to thank family and friends for their emotional support. Your encouragement and faith helped me to continue to work towards achieving this goal. I am most grateful to you! 


\section{TABLE OF CONTENTS}

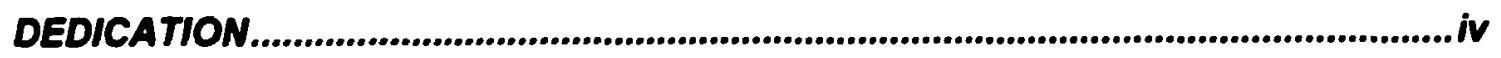

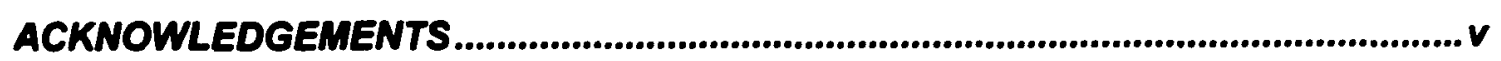

TABLE OF CONTENTS..................................................................................................... vi

LIST OF FIGURES .................................................................................................................

LIST OF ACRONYMS....................................................................................................

ABSTRACT .......................................................................................................................

Chapter 1: INTRODUCTION ......................................................................................................1

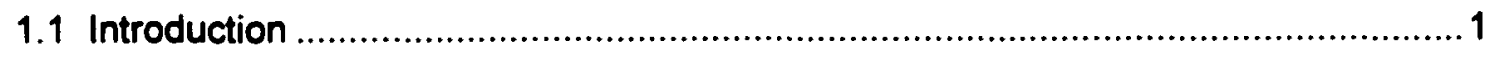

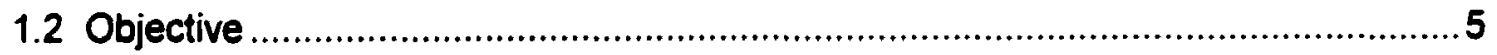

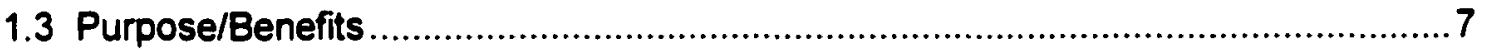

Chapter 2: DEFINING THE PROBLEM............................................................................8

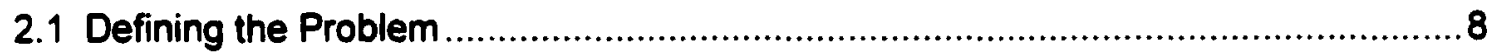

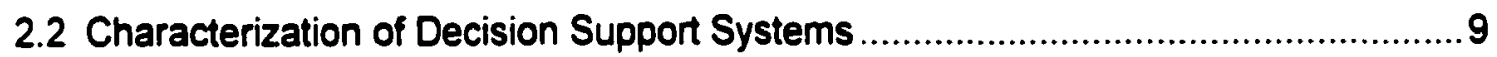

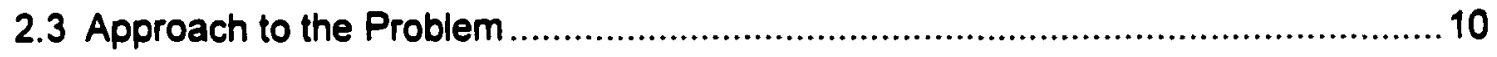

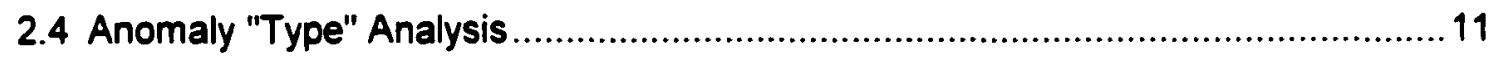

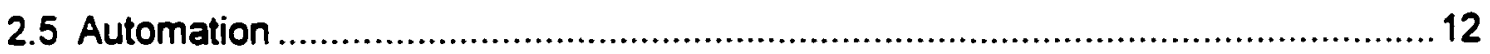

Chapter 3: PRESENT CASE STUDY BACKGROUND ................................................15

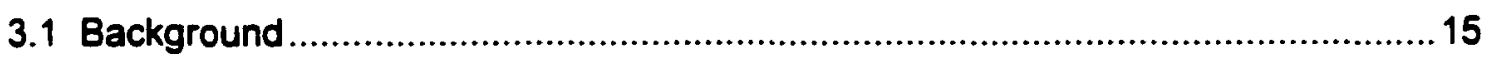

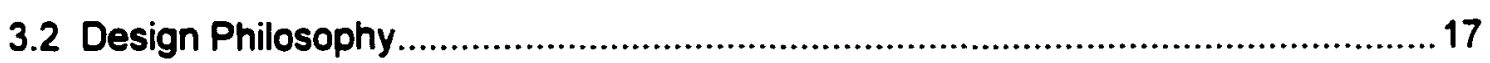

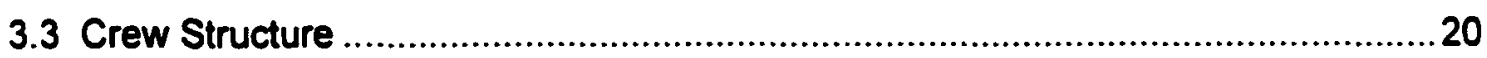

3.3.1 Satellite System Operator (SSO) ........................................................20

3.3.2 Ground System Components (GSO) ..................................................20

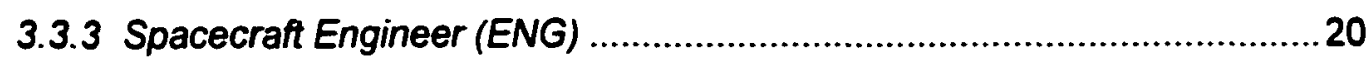

3.4.4 Expert Operator ................................................................................ 21 


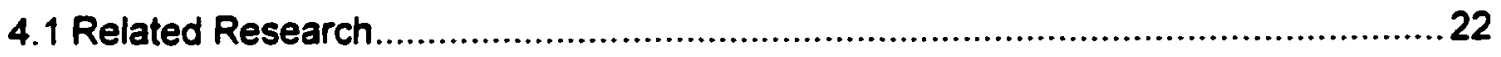

4.1.1 General Considerations …................................................................22

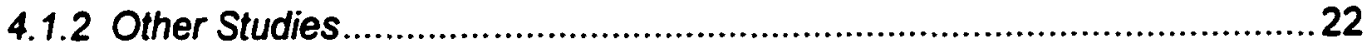

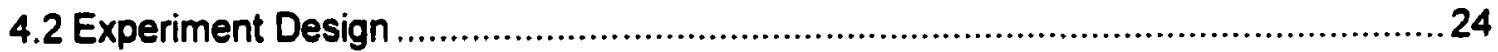

4.3 Resources

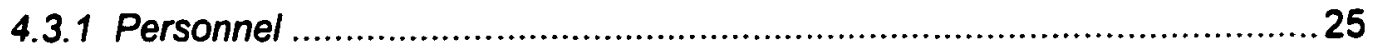

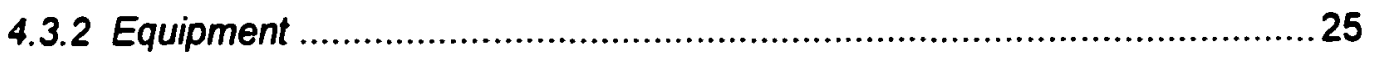

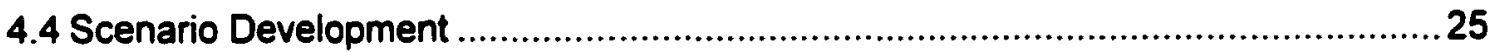

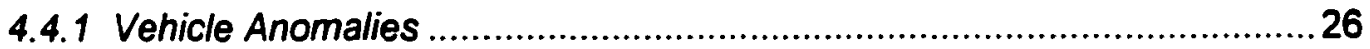

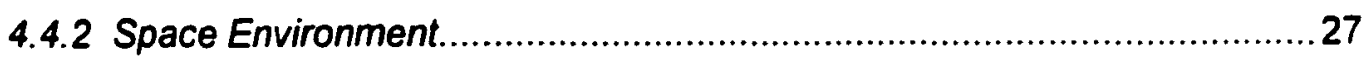

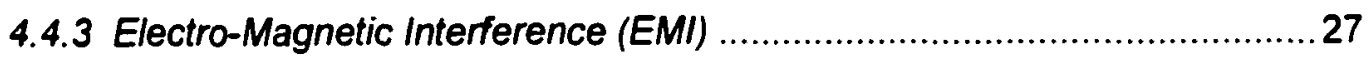

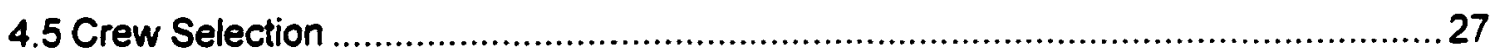

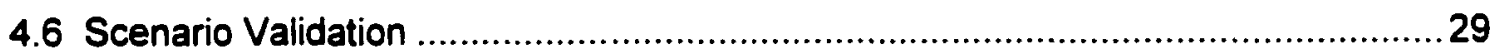

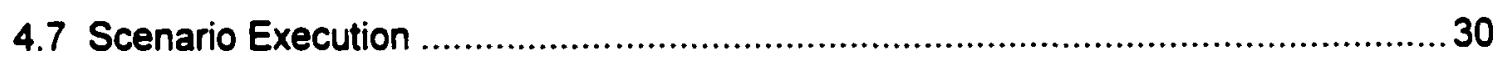

4.7.1 Timekeeping/Recording .................................................................. 30

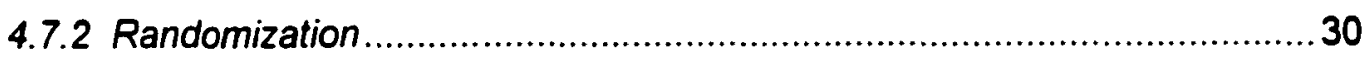

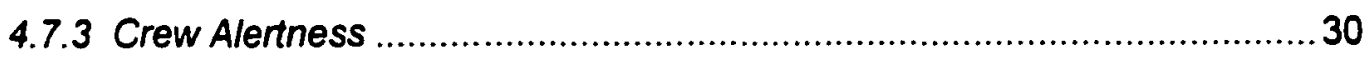

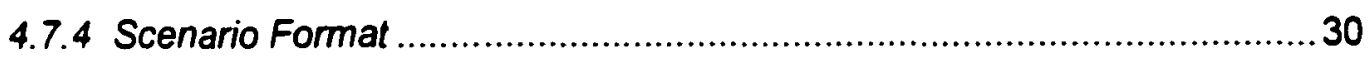

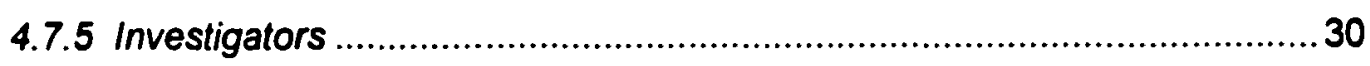

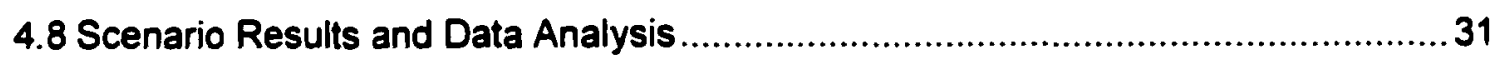

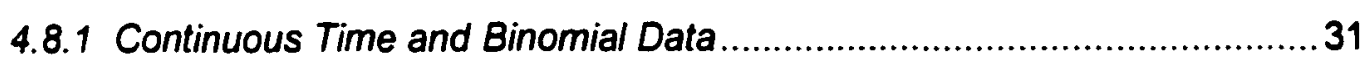

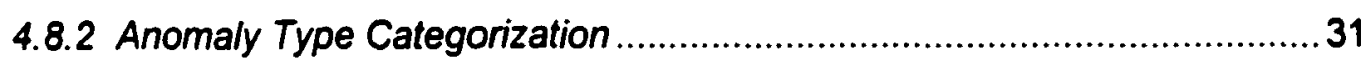

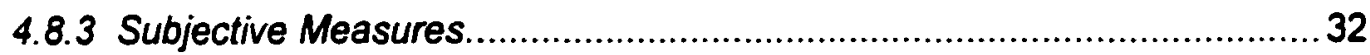

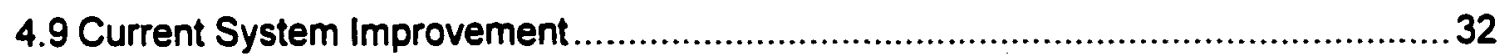

Chapter 5: EXPERIMENT DESIGN................................................................................34

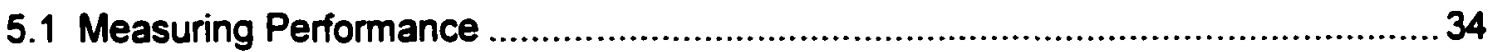

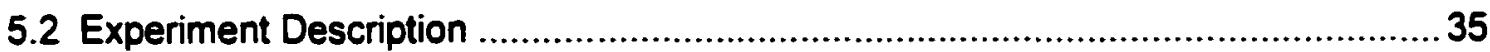

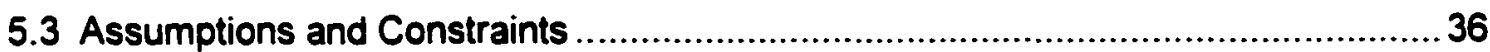

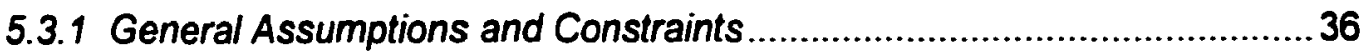

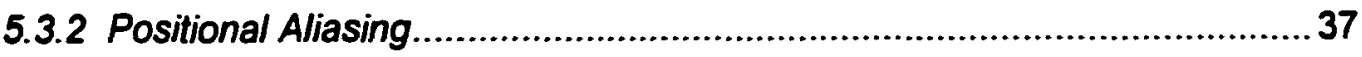

5.3.3 Ground System Operator (GSO) Position .................................................38

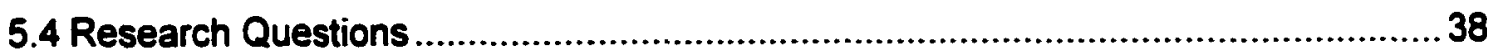




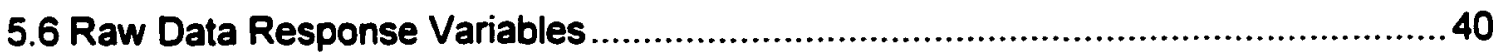

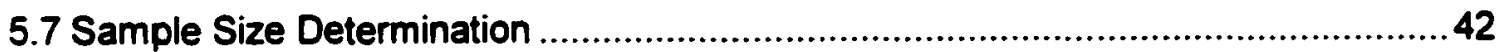

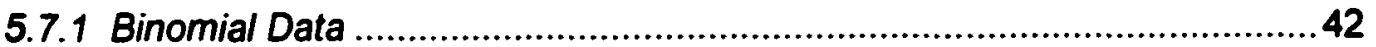

5.7.2 Continuous Time Data ................................................................ 43

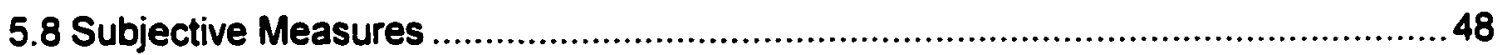

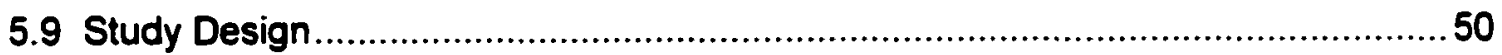

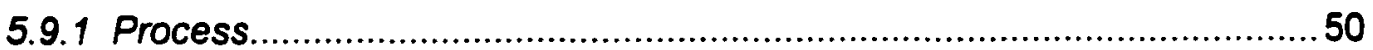

5.9.2 Anomaly Sampling Techniques.....................................................52

5.9.3 Crew Sampling Techniques and Associated Effects ...........................52

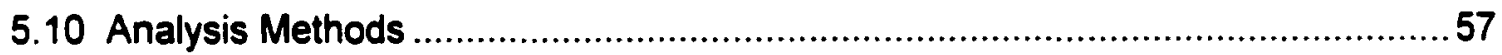

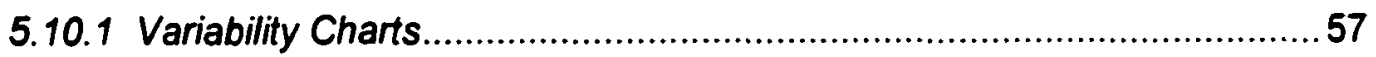

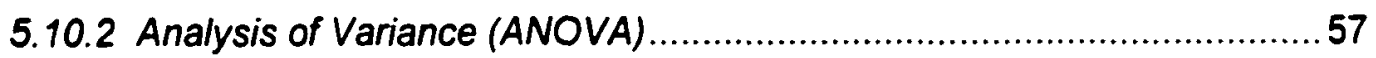

5.10.3 Cox's Proportional Hazards Model (for Continuous Data) ......................58

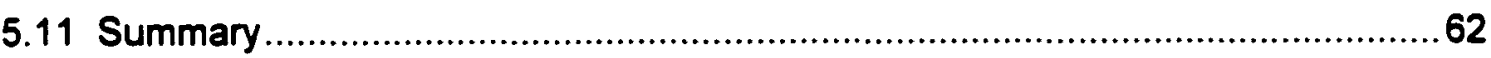

Chapter 6: DATA PRESENTATION AND RESULTS ........................................64

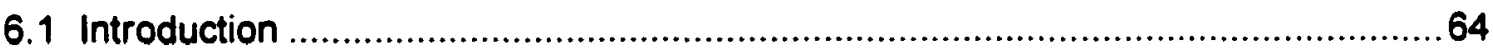

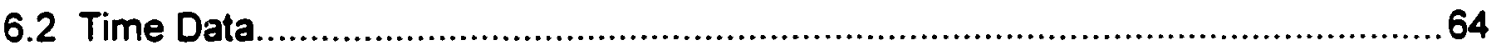

6.2.1 Initial Analysis ....................................................................64 64

6.22 Cox's Proportional Hazards Analysis ................................................ 73

6.2.3 Proportional Hazards Results .................................................... 87

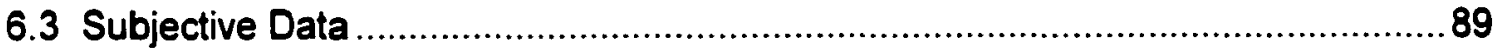

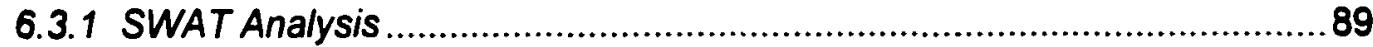

6.3.2 SWAT Data Interpretation ........................................................ 89

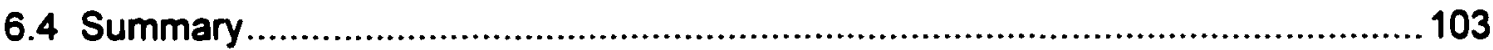

Chapter 7: DISCUSSION/RECOMMENDATIONS ................................................... 105

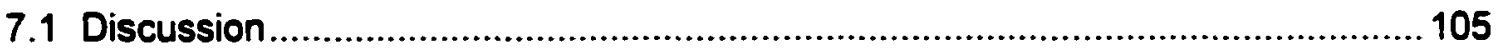

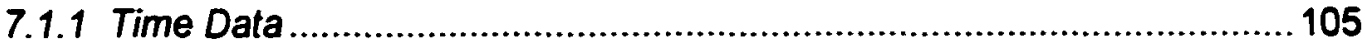

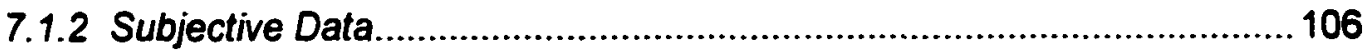

7.1.3 Additional Analysis and Considerations ......................................... 108 


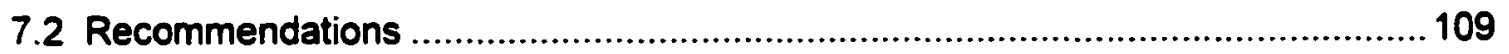

7.2.1 Operators

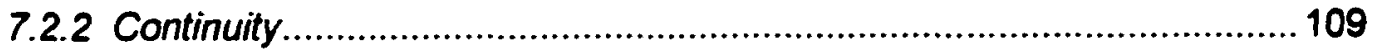

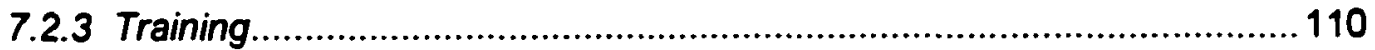

7.2.4 Task Difficulty and Awareness ........................................................110

7.2.5 Personnel Cost Estimates....................................................................111

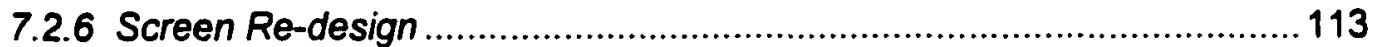

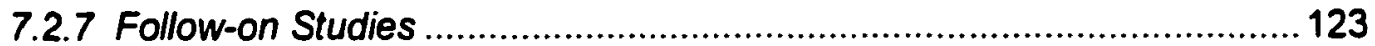

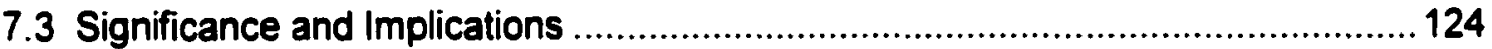

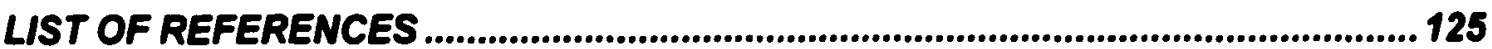

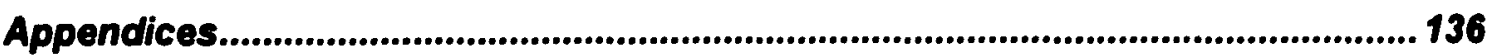

Appendix A: RAW DATA AND STATISTICAL ANALYSIS ....................................... 137

Appendix B: INSTITUTIONAL REVIEW BOARD (IRB) APPROVAL FOR USE OF HUMAN SUBJECTS............................................................... 157

Appendix C: CREW SUBJECTIVE SURVEYS ............................................................. 159

Appendix D: SAMPLE SCENARIO FORMAT ........................................................... 163 


\section{LIST OF FIGURES}

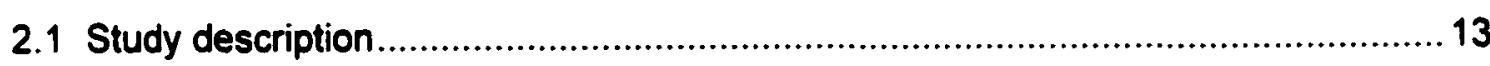

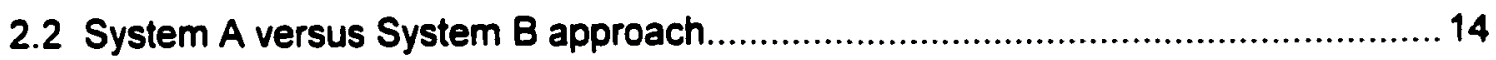

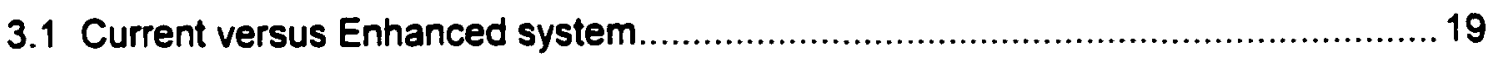

5.1 JMP Sample size determination for comparison of two treatments .....................45

5.2 JMP Sample size determination for comparison of six treatments .......................46

5.3 Sample size determination comparisons for binomial and continuous data ...........47

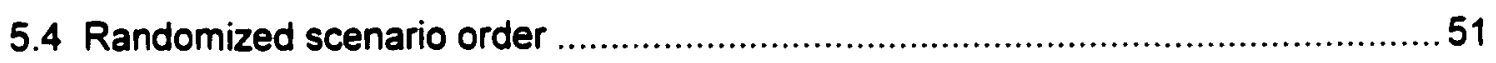

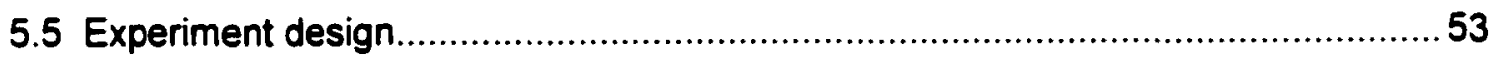

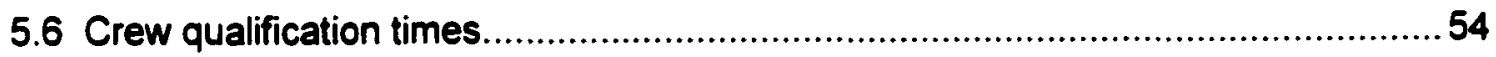

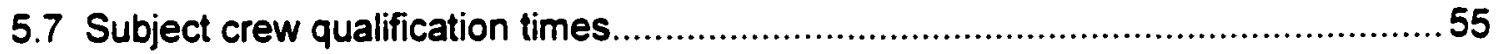

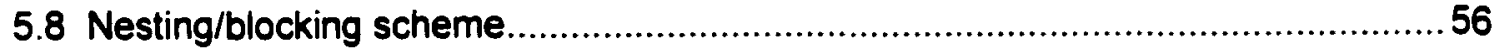

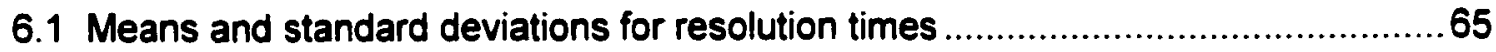

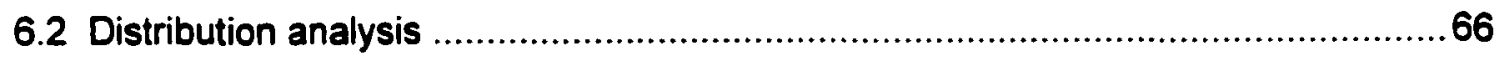

6.3 Lognormal fit for both knowledge levels................................................67

6.4 Variability charts for scenario versus time and $\ln (t i m e)$.................................68

6.5 Variability charts for scenario versus time grouped by crew .............................69

6.6 Variability charts for knowledge versus time and In(time) .................................70

6.7 Variability charts for type versus time and $\ln (t i m e)$....................................... 71

6.8 Random effects of crew nested within knowledge ....................................... 73

6.9 Survival plot of time data grouped by knowledge level...................................... 75

6.10 Survival plot of time data grouped by anomaly type....................................... 76

6.11 Kaplan-Meier and Cox's Proportional Hazards survival functions versus resolution time, combined crews........................................................... 77

6.12 Kaplan-Meier and Cox's Proportional Hazards survival functions versus

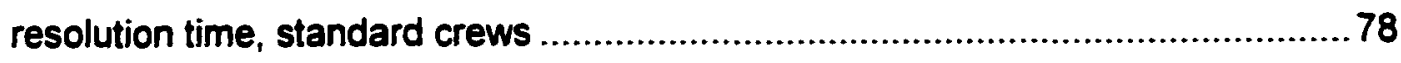


6.13 Kaplan-Meier and Cox's Proportional Hazards survival functions versus resolution time, expert crews

6.14 Log-log transformation of Kaplan-Meier and Cox's Proportional Hazards survival functions versus resolution time, combined crews 80

6.15 Log-log transformation of Kaplan-Meier and Cox's Proportional Hazards survival functions versus resolution time, standard crews 81

6.16 Log-log transformation of Kaplan-Meier and Cox's Proportional Hazards survival functions versus resolution time, expert crews. 82

6.17 Cox's Proportional Hazards model; baseline survival plot (final model) 84

6.18 Risk ratio calculations .89

6.19 SWAT Rescaling. .90

6.20 Prototype analysis and subject correlation. 92

6.21 Goodness of fit of rescaled values. 92

6.22 Overlay plot of SWAT anomaly difficulty by resolution times. 94

6.23 One-way analysis of variance, SWAT rating by type 94

6.24 ANOVA with SWAT rating by type and knowledge 95

6.25 Resolution time by SWAT rating. 96

6.26 Scatterplot of resolution time by SWAT rating for standard crews. .98

6.27 Scatterplot of resolution time by SWAT rating for expert crews .99

6.28 Scatterplot of SWAT rating by resolution time for standard crews..................... 100

6.29 Scatterplot of SWAT rating by resolution time for expert crews ......................... 101

6.30 ANCOVA of SWAT rating by resolution time, knowledge and type. 102

6.31 Correlation and Covariance matrices of SWAT rating by resolution time, expert and standard crews 103

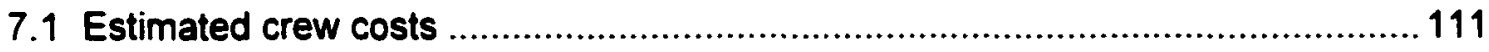

7.2 Estimated crew force and associated costs ............................................. 112

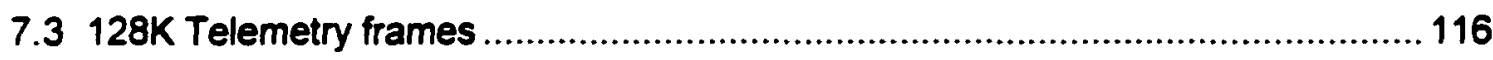

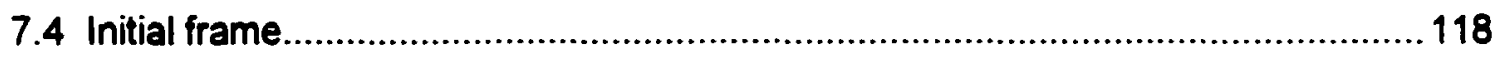

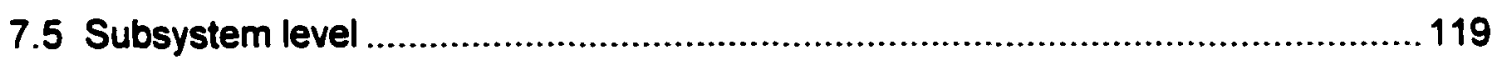

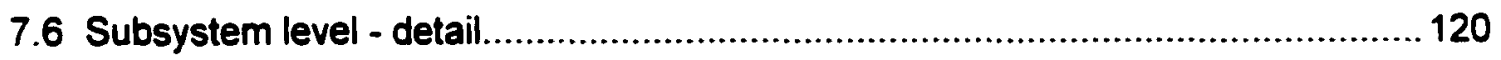

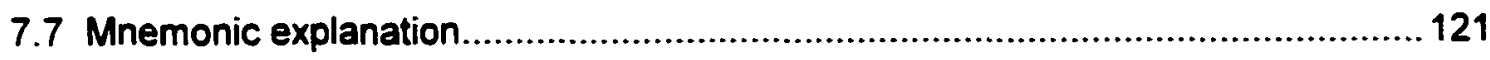

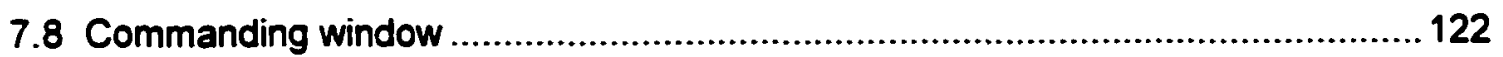




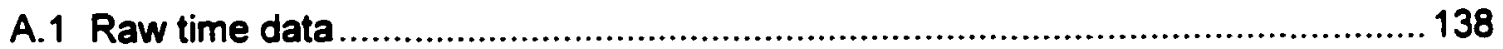

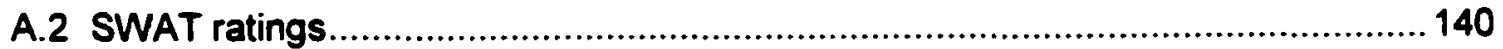

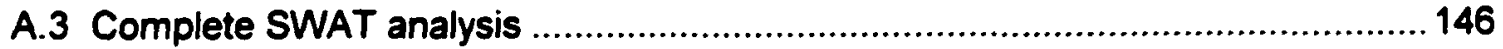

B.1 Institutional Review Board approval letter ........................................... 157

C.1 Subjective measures worksheet ....................................................... 160

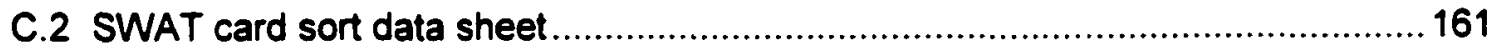




\section{LIST OF ACRONYMS}

1 SOPS $-1^{\text {st }}$ Space Operations Squadron, located at Schriever Air Force Base,

Colorado

AEROJET - Military Space Contractor, Defense Support Program Technical Advisors

AFB - Air Force Base

AFSCN - Air Force Satellite Control Network

AFSPC - Air Force Space Command

ANCOVA - Analysis of Covariance

ANOVA - Analysis of Variance

ARTS - Automated Remote Tracking Station

$C^{2}-$ Command and Control

CERES - Center for Research Support, located at the Joint National Test Facility,

Schriever Air Force Base, Colorado

$\mathrm{Cl}$ - Confidence interval

COA - Course of Action

COTS - Commercial off-the-shelf

CPH - Cox's Proportional Hazards

CTE - Crew Training Emulator

DF - Degrees of freedom

DSP - Defense Support Program

EMI - Electro-Magnetic Interference

ENG - Engineer

GSO - Ground System Operator

HMI - Human Machine Interface

IRB - Institutional Review Board

JMP - Statistical Software Program, SAS Institute

MCH - Modified Cooper Harper Scale

NASA-TLX - National Space and Aeronautics Administration Task Load Index

RR - Risk Ratio

RRCl - Risk Ratio Confidence Interval 
SOH - State of Health

SSO - Satellite System Operator

SWAT - Subjective Workload Assessment Technique

SWORD - Subjective Workload Dominance Technique

TA - Technical Advisor

TRW - Military Space Contractor, Defense Support Program Technical Advisors

UNH - University of New Hampshire

USAF - United States Air Force 


\title{
ABSTRACT \\ HUMAN-MACHINE SYSTEM DESIGN OPTIMIZATION FOR NON-DETERMINISTIC SPACECRAFT ANOMALY DETERMINATION/RESOLUTION
}

\author{
By \\ Kriss Quinn Hunold \\ University of New Hampshire, May, 2002
}

This research examines the dependence of knowledge on decision making with varying levels of uncertainty or non-deterministic situations. The work presented outlines a global approach that is not limited to a specific case study example, but can also be translated to other systems requiring operators, a degree of automation, time constraints, remote control, and high levels of personnel expertise. The specific objective of this study was to address the uncertainty inherent in satellite command and control and to assess and understand the role of human knowledge in the combined human-machine system unit. This research focused solely on the user component of complex human-machine systems. Machine technology level remained constant; no modifications or variations were made to the machine operating system. However, the user knowledge level was altered to examine the effect of this variation and resulting operator ability to troubleshoot and resolve system anomalies.

The case study researched was the Defense Support Program (DSP) satellite constellation currently in use by Air Force Space Command for missile warning. Three different "Types" of tasks were defined, where the three Type categories (1, 2, and 3) represented the level of task difficulty (low, moderate and high). Each task consisted of resolving a unique satellite vehicle anomaly within pre-scripted scenarios. 
The role of human knowledge was examined and found to be significantly

important. This result was more evident as the situation uncertainty or complexity of the task increased. This data may be useful to continue the optimization of both user and machine to create a human-machine system capable of adapting to the rapidly changing space environment and able to contribute more fully to tomorrow's space control objectives. 


\section{Chapter 1}

\section{INTRODUCTION}

\subsection{Introduction}

The roles of machines and humans in space systems have perplexed system designers since the advent of their use in early 1960's. Space systems are challenging systems to study since the human-machine system occurs remotely over a distance of thousands of miles, where the earth-based user never visually connects with the in-flight system they are monitoring and ultimately affecting. In essence, there is always a "correct" solution to a satellite problem, assuming all data is complete. "Correctness" is indicative of the exact, definitive solution necessary to remedy the satellite problem. However, the realization that oftentimes the data is incomplete (or non-deterministic) presents an entirely new set of problems to the satellite controller. To compound the issue, satellite anomalies are only evident by examination of the telemetry displayed through the operator consoles on ground.

Air Force Space Command (AFSPC) has been wrestling with determining the most efficient and cost-effective method of performing satellite command and control $\left(C^{2}\right)$. As AFSPC moves towards the goal of space control, the problems posed to the satellite operator become increasingly more non-deterministic, meaning that they are more complex, foreign, and often associated with incomplete data where the anomaly cause cannot be conclusively assessed. In anticipation of how satellite command and control will evolve in the future, it seems evident that there will continue to be increased 
complexity and uncertainty present in the space environment, requiring more adept problem solving expertise than ever before.

The systems design approach presented here will involve a case study of one particular space system where the roles of human and machine could be adjusted to create a better overall unit to perform satellite commanding and control. This is increasingly important when impacted by data uncertainty. Since most systems have components of both a machine and human working synergistically to achieve a common goal, it is important to address both components thoroughly during the system development phase.

Customarily, engineers and scientists are more familiar or comfortable with the machine portion of design since it is usually predictable and easily understood. Conversely, determining the "best operator" to design a system for can be a laborious and subjective process; human metrics can be difficult to assess and quantify. In this study, the "best operator" can be described as that person who can perform all necessary on-orbit functions, to include ground configuration, satellite command and control, engineering, and anomaly troubleshooting. They have capacity for cognitive skill and intuition, and can operate autonomously in performing problem diagnosis and resolution. The "best operator" can operate in presence of uncertainty and with no direction. In addition, they can perform tasks with minimal or no supervision, and in situations where no prior contingency planning exists.

The overarching system design process initiated in this study (in combination with future research) will strive to accomplish two objectives:

1) Determine the operator knowledge level (derived from training and experience) best suited to complete the task at hand when combined with the machine side \{within the scope of this study\} 
2) Create an intelligent, intuitive and effective interface for that operator to work with \{future research\}.

The interface is extremely important, since it is the sole tool that facilitates effective and accurate troubleshooting, implements the display of the necessary telemetry data, and promotes operator vigilance in detection of anomalous situations.

The approach used in this study is to investigate the trouble-shooting success rates of different operators of varying knowledge levels. The test results obtained from studying these operators of different skill levels may be used to guide what design strategies, machine enhancements and automation can be incorporated in a new satellite control system. Future machine enhancements for a current satellite control system could include automation of certain deterministic commanding, or at a minimum, state of health (SOH) anomaly notification. The new system can guide either the operator, the computer, or operator aided by the computer through a sequence of actions to solve problems, troubleshoot anomalies, or perform routine or emergency commanding. The responsibility of consequential decision making will often lie with the user; however, the machine will provide some guidance to its human counterpart to aid the operator in making a decision and to ensure that irreparable damage is not caused to the on-board systems. With user and machine working cooperatively in this manner, human error can be minimized. Consequently, superior human abilities of problem solving and decision making are strengthened by the computer's capabilities of raw data filtering and interpretation, thus allowing the human to be used more efficiently.

According to Sarter in his studies of the Airbus A-320 (Sarter, 1997), "advanced automated systems required a high degree of authority and autonomy. Their resulting agent-like behavior required increased coordination between human and machine to ensure that the system operator maintained a high level of awareness of the activity and intentions of the automated system." This indicates that the awareness and intuition 
level required by the operator also supports an individual with experience and training. This is especially important in situations where "increasing knowledge demands, coupled with static or decreasing training investments, can easily lead to significant gaps or misconceptions in user's mental models of the automated systems they managen (Sarter, 1997). Intuitively, this would also confirm that automation does not give license to selecting a minimally trained or inept operator. The converse appears true; when the human and machine are integrated more closely, the operator must interpret the machine at a high level and understand the consequence of an incorrect action. Furthermore, the operator should have the capacity to override the machine and troubleshoot the situation if an incorrect automation sequence is suggested.

There are several interrogation methods for determining satellite health. Optical telescopes can assess satellite position and physical configurations, but coordination to use these assets requires time that would extend past the point where intervention could avert such anomalies. In addition, only external satellite health (tumble, solar panels, exterior damage) can be assessed by optical means. Satellite telemetry is the method most readily available to determine the overall (external and internal) health of the satellite. However, if mechanisms such as transmitters, receivers and commanding links on board have failed, the vehicle is unable to provide data. In addition, telemetry is not always completely accurate and reliable due to jamming, interference, data link anomalies, and internal component failures.

This study will investigate how humans interface with machines in lieu of incomplete or missing data, which is a typical occurrence when dealing with remote systems. Since there is no visual contact with the vehicle and the true state of the satellite cannot be ascertained, one must act on assumptions, knowledge, or prior experience based on the data presented. The decision options tend to have different levels of confidence associated with each. Uncertainty is added to the decision making 
process by means of spacecraft subsystem anomalies, the overall space environment (geomagnetic status, solar wind, and solar storms), and satellite control interference (unintentional or deliberate).

\subsection{Objective}

The following study objective outlines a global approach that is not limited to satellite vehicle command and control, but can also be translated to other systems requiring operators, a degree of automation, time constraints, remote control, and high levels of personnel expertise.

This research will focus solely on the user component of satellite command and control. Machine technology level will remain constant; no modifications or variations will be made to the machine operating system. However, the user knowledge level will be altered to examine the effect of this variation and resulting operator ability to troubleshoot and resolve satellite anomalies. Two different levels of operator knowledge will be examined, where the term knowledge in the study represents the level of training and experience held by a particular user. The two knowledge levels used are identified as standard and expert.

Standard level: describes a fully qualified operator currently performing satellite operations in the case study satellite system. These operators were trained to the level of training and experience currently baselined and approved by Air Force Space Command. The "mean time qualified" for these individuals was less than two years, and the training period generally lasts for two to three months.

Expert level: describes a fully qualified operator able to perform all functions of satellite operations in the case study system. These operators were trained 
beyond the current, approved training baseline and have extensive engineering knowledge of the satellite system. These operators are recognized by Air Force Space Command as experts, and are designated as such with the title "Technical Advisors". For the purposes of this study, the "mean time qualified" for these individuals was defined as over four years of training and experience.

In order to define the expertise needed to perform various tasks associated with anomaly resolution, different task levels, or "Types" will be examined. This process involves categorizing many of the possible anomalies an operator might encounter into Types 1, 2, or 3 based on complexity and resolution difficulty. Each task type is associated with an increasing level of complexity of satellite anomaly. This process may enable leadership to ascertain what level of knowledge is necessary to resolve certain types of anomalies. If a certain level of risk is acceptable, then perhaps decision makers would prefer to have operators capable of resolving only a Type 1 or 2 anomaly as their "standard", translating into a specific individual possessing a distinct level of training and experience.

This study focuses on varying the operator experience and training level only, and keeping the machine portion of the system constant. Although the end result of system optimization would require variation of both components, it is useful to assess each entity separately to gain understanding of the human-machine system. Follow on research should focus on optimizing the machine interface by varying technology and automation while maintaining one consistent knowledge level during the test process.

The following chapters will detail the history of this problem within the military satellite control field. However, this issue is very applicable to many disciplines in commerce and industry. In addition to the background discussion, methodology used to construct the experiment and statistical results will be presented. 


\subsection{Purpose/Benefits}

This project could greatly benefit Air Force Space Command (AFSPC) by contributing a concrete study with objective data and statistical results. The comparison of current operator knowledge level and crew structure to a smaller, expert case-study crew is extremely relevant and valuable, and could facilitate objective conclusions on structuring tomorrow's satellite operations crew force. The understanding of the humanmachine system gained from this project could be employed in planning for a future force able to execute space control missions with increased overall effectiveness. Moreover, this effort stands to benefit the Center of Research Support (CERES) as a test bed for external interfaces, and would be their first venture into cooperative research efforts. Contributors to this effort were comprised of a University of New Hampshire (UNH) doctoral candidate, active duty Air Force operators from the $50^{\text {th }}$ Space Wing $/ 1^{\text {st }}$ Space Operations Squadron, Air Force Reserve operators from the $7^{\text {th }}$ Space Operations Squadron and personnel from every discipline at CERES. 


\section{Chapter 2}

\section{DEFINING THE PROBLEM}

\subsection{Defining the Problem}

Optimization of the entire human-machine unit is a process that must occur incrementally. This study concentrates on the human portion of the system, and subsequent research will focus on optimizing and investigating the machine component. Spanning both of these elements is the fundamental problem of how to balance the machine and human in large, complex systems. Clearly, if all aspects of a particular system can be accurately defined and characterized, then this optimization approach can be universally applied to any systems having these same attributes. These characteristics will be investigated and discussed in the chapter, after which knowledge will be added to the systems engineering understanding for these decision systems. If we consider two separate but identical systems and add knowledge to one, at what point does complexity of inputs create a variance between them?

Optimization will also involve supplying final data to be used for research continuation at the Center for Research Support (CERES), located at the Joint National Test Facility at Schriever Air Force Base, Colorado. CERES is working to create informative operator displays by means of indicating failure points and anomalous situations, in addition to automation of routine, deterministic "Type 1 " level anomaly commanding. Automation, used properly, frees the operator to focus attention on nondeterministic problems, troubleshooting or more complex anomalies (Types 2 and 3). 


\subsection{Characterization of Decision Support Systems}

The Decision Support Systems of interest are two-part systems characterized by the following attributes:

A. Human-Machine Component

1) The portion comprised of a human and machine element, where:

- The human brings experience or knowledge

- The machine provides decision support and automation capability

2) The machine portion of the system can help identify the problem and provide a potential corrective action for the complex system

3) The decisions are tightly constrained in time

4) Decisions must be extremely precise and made with a high level of confidence

B. Operating System Component

5) Limited or incomplete data (or information/knowledge) is injected into the decision support system from the operating system

6) The operating system is remote, meaning there is no visual confirmation of the true problem and the corrective effect

7) There exists a "correct" answer for a problem under consideration given the real input. If complete data was always obtainable, one would know the right action to take when resolving anomalous situations. This also implies we are dealing strictly with physical systems. One limitation is that occasionally situations exist where the problem canno! be successfully repaired (e.g., battery explosion); however, safing actions may still be implemented. 


\subsection{Approach to the Problem}

It is possible to construct a "System A versus System B" approach to measure the effectiveness of the existing system with that of an optimized system. In these systems we have two portions: the Operating System (the satellite vehicle) and the Human-Machine (or Decision) System. The Operating System is assumed to be a deterministic system, whereas the Decision system is non-deterministic in nature due to missing or incomplete data. The theory is given a series of inputs that are varied according to the number, rate, and complexity, we can examine how well each system (A and B) can identify each anomaly and correct it. The measures of success are the number of errors committed, the respective personnel costs of operation (in number of persons required to do the job), efficiency (time required to properly identify and correct the problem) and accuracy. Figures 2.1 and 2.2 display a schematic of how a system $A$ versus system $B$ approach might be viewed.

Assessing number of errors can have several implications. Simply stated, this can be determining the number of incorrect commands sent or delays in commanding the satellite. However, repercussions of physically reducing the number of operators had to be contemplated. One effect of operator reduction is possible lack of vigilance and effectiveness in the event of operator illness, fatigue, operator unavailability and similar crises. The benefit to having multiple operators on console is redundancy and additional monitoring capability. Yet, this benefit is extremely minimal since currently there is little overlap in crew functionality. There could be some benefit for error reduction with the current scheme of two Satellite System Operators as outlined in Section 3.3. However, there is almost no sharing of knowledge, function, or expertise between the positions of Engineers, Ground Systems Operators, and Satellite System Operators. 


\subsection{Anomaly "Type" Analysis}

It is important to try to define knowledge levels so we may better grasp what it means to require an expert level of knowledge versus a standard level. One approach is to predict problem "Types" by examining as many potential problems or anomalies that can be encountered by the human in the system. These types can be defined in the following way:

1) Type 1: Simple complexity tasks. These tasks could possibly be automated, needing only minimal, if any, operator intervention. Type 1 typifies routine tasks.

2) Type 2: Medium complexity tasks. These tasks can be accompanied by some degree of automation capability, with the operator providing guidance for the tasks. Type 2 tasks are more difficult to resolve than those tasks that are characterized as "Type 1 " tasks and characterize function tasks such as switching components and altering satellite configurations.

3) Type 3: Difficult complexity, non-deterministic tasks. These tasks would probably not be well suited to automation, but could be expedited by a high degree of machine data filtering and advanced data presentation. In Type 3 tasks, subtleties in data could possibly provide indications of multiple anomalous conditions, and are today typically characterized today by very rare and atypical anomalies. However, it is anticipated that future satellite operations situations will consist of infrequent but critical complex tasks characteristic of Type 3 anomalies.

Details pertaining to the statistical study and Type categorization can be found in Chapter 5 (Experiment Design). 


\subsection{Automation}

The benefits of incorporating automation into satellite command and control have been mentioned periodically in this document. However, some observations on the subject of automation should be considered. Occasionally, extensive automation can result in a out-of-the loop performance problem, causing a potential negative consequence (Billings, 1991; Moray, 1986; and Wickens, 1992). According to Endsley et al. (Endsley, et al., 1995), "system operators working with automation have been found to have a diminished ability both to detect system errors and subsequently to perform tasks manually in the face of automation failures, compared with operators who manually perform the same tasks." Essentially, an important automation concern is the loss of manual skills or focused attention which accompany the introduction of automated tasks. Therefore, while some tasks can be comfortably automated, care must be taken to not mentally remove the operator from their environment as to facilitate passivity or loss of diligence or vigilance. 
HUMAN -MACHINE SYSTEM DESTGN OPTMIZATION FOR NONDETERMINISTIC SPACECRAFT ANOMALY DETERMIMATION/RESOLUTION
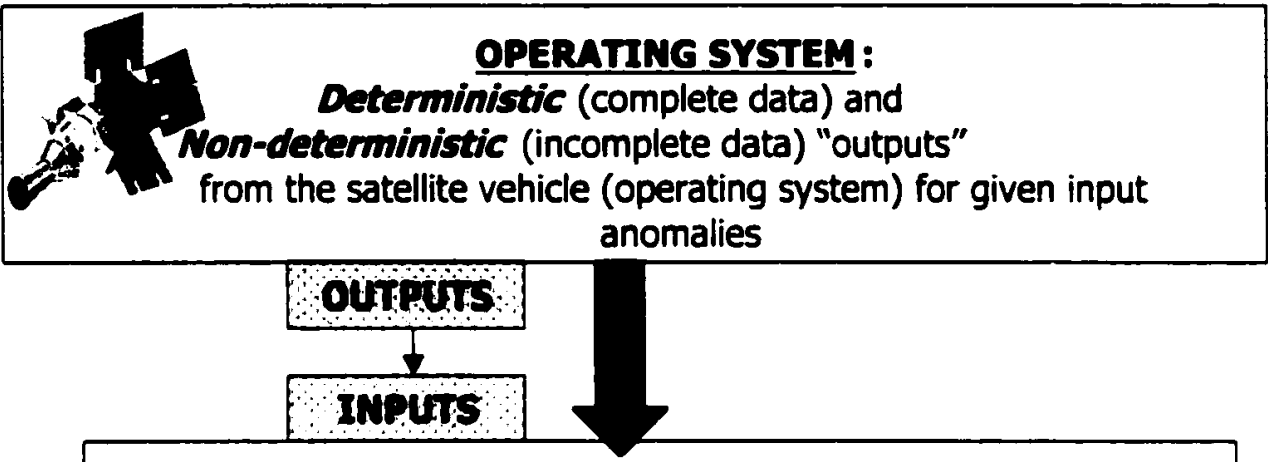

HUMAN-MACHINE DECISION SYSTEM: "Outputs" provide "inputs" to the Human-Machine System Phased Approach:

- Fix level of machine technology

-Vary knowledge level

TASK: Identify anomaly/anomalies and corresponding signatures and give correct response

$\begin{array}{ll}\text { Measurea } \longrightarrow & \text { Derive: } \\ \text {-Errors } & \text {-Accuracy } \\ \text {-Time to success } & \text {-Efficiency } \\ & \text {-Cost }\end{array}$

SYSTEM LEVEL: Correlate knowledge level in the Defense Support Program (DSP) Command and Control Human-Machine System to decision success for given satellite outputs

PINAL RESULT: Increase understanding of Human-Machine System performance with respect to the factor of human knowledge in varying data/information rich environments 


\section{Figure 2.2: System A Versus System B Approach}

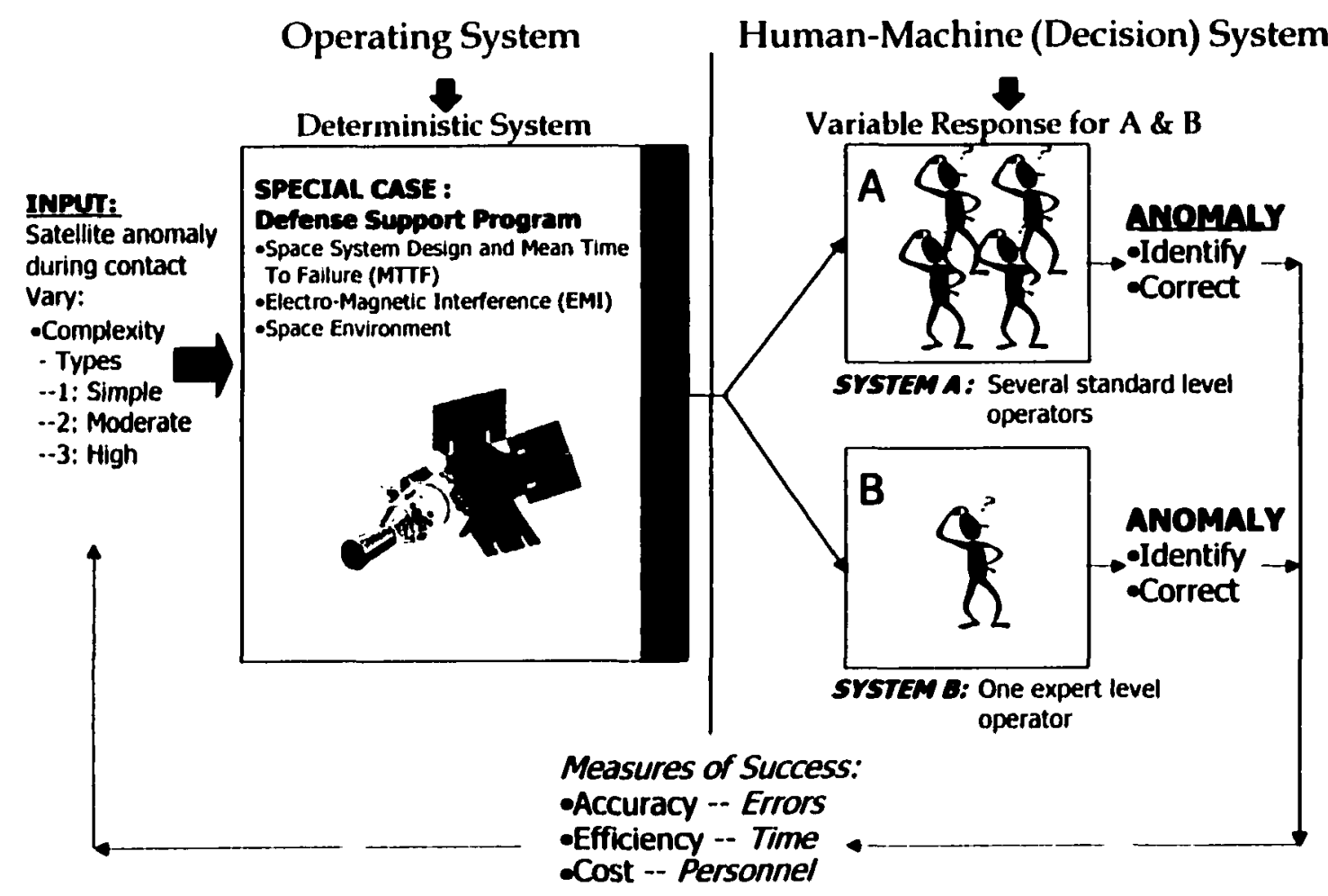




\section{Chapter 3}

\section{PRESENT CASE STUDY BACKGROUND}

\section{AN EXAMPLE: Defense Support Program (DSP) Satellite Constellation}

\subsection{Background}

The case study satellite system is the Defense Support Program (DSP). This constellation is the primary tool used for detection of missile launches and space vehicle launches for North America. The United States and Canada rely on these satellite vehicles for National Security and population defense, making this arguably one of the most critical space programs in our military's possession. To provide full earth coverage, several vehicles hover in geostationary orbit, roughly 22,000 miles above the earth's surface. They detect infrared signatures during missile or space launch boost phases and relay this information to various sensor sites and command centers around the world. The data is then collected from the sites and evaluated by the Missile Warning Center, North American Aerospace Defense (NORAD) Command located at Cheyenne Mountain Air Force Station in Colorado, where the threat to North America is assessed and distributed to national leadership.

The controllers for this satellite constellation are responsible for performing routine checks of the vehicle subsystems via the satellite State of Health (SOH). They also perform all satellite commanding, to include orbit adjustments, subsystem turnon/off, and emergency commanding. Most of these subsystems are critical to the health and mission performance of the satellite, and failures can result in total mission loss or irreparable vehicle damage if not handled correctly and efficiently. The satellite 
operators are responsible for ensuring that DSP is physically capable of providing the first indications of nuclear attack.

Machines are inherently superior at pattern recognition (color and data), datarange checks and data sorting; in the past, older technology systems required the human to perform "data supervision" or caretaking. New technology has minimized or negated this requirement by improving data display and decision support techniques, freeing the human to contribute to the system their unique cognition, intuition, experience and troubleshooting abilities. This human knowledge component potentially becomes more vital, which is why this study examines the dependence of knowledge on decision making with varying levels of uncertainty or non-deterministic situations.

Driskell and Olmstead (1989) describe the military as "the largest employer of initially unskilled labor in the nation. Unlike civilian organizations, the DoD does not always hire personnel with the required skills. "Personnel are trained and assigned to appropriate positions, and these individuals will serve only three to six years in that position" (Weimer, 1996). This fact poses a potential problem in the space operations community, where the jobs are highly technical and complex. In many cases, personnel do not attain a high skill level until the end of their assignments.

Graine (1988) reported that 60 percent of the weapon system life cycle costs are related to people or training requirements. His conclusions, based on total cost, disagree with the theory of high operator knowledge being necessary. Weimer (1995) continues this argument by inferring that considerable savings can be attained if the design requirements specify: 1) reduced crew sizes and 2) a reduction in the skill required to operate and maintain the system. This method may be adequate for simplistic, deterministic systems. However, as the inherent complexity of the system increases (air and space systems) and the data becomes more non-deterministic, operator knowledge could become even more vital. Although crew reduction may be a 
possible outcome of this experiment, the knowledge required to effectively control the system should not be compromised.

When discussing systems of systems (non single-string systems), military systems pride themselves on high levels of redundancy to ensure mission success. This proves more critical in field operations where survivability is paramount. Luckily, it is much easier to build redundancy and survivability into space systems where additional personnel are always co-located with the system and new technology enables automated commanding and monitoring.

\subsection{Design Philosophy}

This study will test the "Enhanced" versus "Current" or standard system (Figure 3.1). This enhanced version involves one expert operator (4+ years experience and knowledge in all satellite areas) against the current manning scheme with the standard crew of four personnel (1 to 2 years), each with specific knowledge areas. These knowledge area include the following: two SSO's, or Satellite System Operators (two are required operationally for vehicle commanding; one is required for state-of-health only), one Ground Systems Operator (GSO) and one Spacecraft Engineer (ENG). The test will be concerned with assessing how the two systems perform in terms of accuracy, efficiency, and personnel cost. Accuracy represents the number of errors committed or inability to resolve satellite problems. Efficiency is synonymous with time to resolution, and the number of operators required to do the job is a contributor to system cost. However, it is important to reiterate that this research does not constitute a crewreduction exercise; it is a study of operator knowledge.

The two primary crew functions that will be studied are: 1) satellite State-ofHealth (SOH) and 2) planned and contingency commanding. The SOH is performed each time the ground station is scheduled to have "contact" with the vehicle before any 
action, such as commanding, occurs. Commanding can either be planned (battery reconfiguration for eclipses, switching subsystem resources) or contingency based (subsystem malfunction, other anomalies).

The satellite SOH process involves a series of approximately seven computer displays that present the data in either color-coded mnemonics (representing various subsystem components, such as battery temperatures, etc.), telemetry patterns, pure numerical values, and numerical ranges. Due to extensive data saturation and human involvement in the data monitoring process, there is present risk of human error with the current design. Although not a part of this research, one consideration for system redesign involves displaying only out-of-limit data points; points within limits are considered nominal and could be eliminated from the initial viewed display (Chapter 7). However, as there are no display limitations, the operator will always have the ability to access and display any telemetry data desired. This will greatly minimize the time and personnel required for routine status checks for anomalous conditions.

For satellite commanding, command plan checklists exist for several possible anomalies or system reconfigurations. These command plans involve the operator manually typing and sending each command to the satellite. The incorrect command sent at an inopportune time can have disastrous effects on the vehicle. This risk could be mitigated by methods that will be discussed in later chapters. 
Possible Inputs:

- Schriever Control (n/a - controlled)

- AFSCN (n/a - controlled)

- Satellite (variable)

- --Actual anomaly

- --EMI

- --Space environment IMPUTS

\begin{tabular}{|c|c|c|c|}
\hline $\begin{array}{l}1 \\
2 \\
\cdot \\
\text {. } \\
\text {. }\end{array}$ & $\begin{array}{l}\text { MACHINE } \\
\text {-Displays data } \\
\text { - Highlights data out of } \\
\text { range } \\
\text {-Identifies bad commands } \\
\text {-Allows commanding } \\
\text {-Provides message } \\
\text { feedback }\end{array}$ & $\begin{array}{l}A \\
A \\
C \\
U \\
R \\
R \\
E \\
N \\
T\end{array}$ & $\begin{array}{l}\text { HUMAN } \\
\text {-Crew of standard (current) } \\
\text { experience (SSO, GSO, ENG) } \\
\text { - Responds to out of limits points } \\
\text { and executes checklist } \\
\text {-Adds engineering knowledge } \\
\text { when greater expertise is } \\
\text { required } \\
\text {-Crew has baseline training } \\
\text { knowledge level; limited trouble } \\
\text { shooting capability }\end{array}$ \\
\hline
\end{tabular}

\section{Eoure 3.1: Cument Versus Enhanced Sxytem}

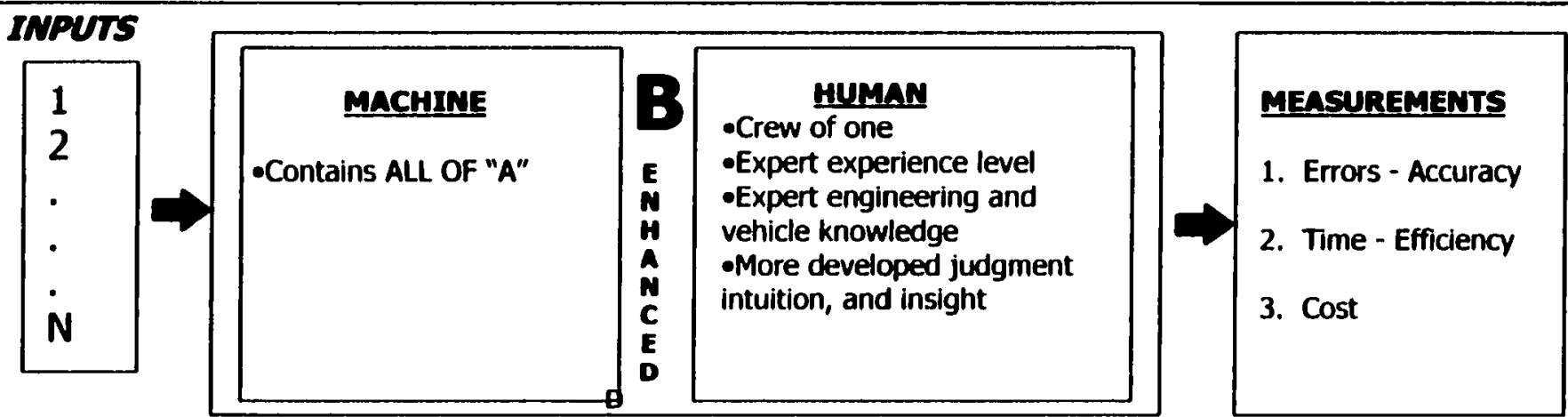




\subsection{Crow Structure}

The crew structure consists of a minimum of three people for a nominal commanding support. For anomalous conditions, an engineer is added to the "crew", making the total crew requirement of four. In this study, the standard level crews will consist of the four individuals whose positions and associated responsibilities are detailed in the following sections.

3.3.1 Satellite System Operator (SSO). The SSO is liable for any problems that arise with the satellite. This individual is responsible for monitoring the satellite state of health and subsystem operability. The SSO's talk directly to the ARTS (Automated Remote Tracking Station) operator to verify that command uplinks and satellite downlinks are active and data is being received. This is the only position permitted to perform direct commanding to the satellite. There is only one SSO required for a nominal SOH support, and two required for any commanding support.

3.3.2 Ground System Components (GSO). The GSO does not interface with any of the satellite components, but instead ensures that the data and voice links from the worldwide Automated Remote Tracking Station (ARTS) network to the local terminals are operating properly. In the scenarios constructed for the study, the GSO position has nominal interface with the ground segment; no variation or additional inputs were presented within the ground equipment.

3.3.3 Spacecraft Engineer (ENG). The engineer is the Air Force's resident satellite system expert. They reside on station for roughly 3 years (the first year is spent in training), so the expertise gained is limited to witnessed anomalies, on-the-job training 
and knowledge passed from senior engineers. The engineer is present during day shift, but is also on 24 hour call for anomalies and problems. The time criticality of some failure resolution makes this a less-than-optimal situation for the crews when performing contingency anomaly troubleshooting. The engineers, in most cases, consult with onsite contractor personnel during anomaly detection. In addition to the local contractors, there is occasionally a need to confer with the production plant satellite subsystem specialists and designers.

3.4.4 Expert Operator. The "expert" operator for this study had a mean experience level of seven years. This level of expertise included mastery of the GSO, SSO, and ENG functions. Additional skills included mission planning, orbital analysis, and detailed system knowledge. They could act autonomously in reconfiguring the satellite and substituting redundant components. Their knowiedge encompassed not only the satellite bus, but all vehicle payloads.

The system comparisons, crew design and assumptions and constraints associated with the design will be detailed in later chapters. 


\section{Chapter 4}

\section{METHODOLOGY}

The methodology outlined in this section describes the various phases followed in the study development.

\subsection{Related Research}

4.1.1 General Considerations. It was determined that a number of scenarios would be run between two crews of different knowledge levels (expert and standard) and performance factors would be compared. However, the steps below had to be followed prior to experiment design.

- Ensure parameters are clearly defined and ascertain which are constant or variable

- Verify that objectives are testable and establish criterion of success for each item

- Examine hypotheses, determine validity, and verify good test cases

\subsubsection{Other Studies. Background cases and studies were also examined to see how} well they fit within the guidelines of this research. There have been many operator studies performed, but few of them fall within the purview of the objectives stated in Chapter 1.

Jones and Mitchell (1995), in their research of Goddard Space Flight Center Satellite Flight Operations Teams, collected performance and subjective data from the crews on satellite ground control tasks. Eight subjects were presented seven scenario sessions, where the main variable was the absence or presence of the operator 
assistant software designed to aid the operator in task reminders and decision making. Their experiment held operator knowledge constant and level of automation and assistance provided by the system as variable. Results found that most operators were surprisingly confident in allocating tasks to the software. Some reasons for this phenomenon were that operators were aware that in a simulation environment there were no consequences for poor decision making. Also, they were cognizant that one objective was to test the software and its capabilities. The software exhibited no failures in interface or automation and performed functions with virtually no time delay. In sum, the researchers showed that the assistant software was perceived by the operators to "be useful and provided benefits for certain portions of the control task".

Kerstolt et al. (1996) performed a similar operator study with disturbances rated on complexity where either of two options were available: one disturbance was present (complexity 1), or two or three simultaneously (Complexities 2 and 3). Limited time was available to resolve these anomalies. However, their subjects were all inexperienced novices and were of the same knowledge level. Kerstolt's group demonstrated that participants had tendencies of ignoring the monitoring functions as disturbances or anomalies occurred. Additionally, there was evidence of what the researchers described as "cognitive lockup": once complex diagnosis began, operators ignored the opportunity to stabilize additional, simpler system faults and tended not to interrupt the original troubleshooting process.

Elvers et al. (1993) produced a factorial experiment similar to the one presented in Chapter 5 (Experiment Design), measuring reaction time and absolute error with students of the same level of knowledge. This variation is similar to that of Kerstolt's (1996) study, concentrating on overall performance with no variation of operator knowledge. The research focused on operators performing tasks requiring either information integration (integral) tasks or separable tasks, and probabilities of integral 
task occurrence were controlled. Results showed that when the probability of integral task was increased, absolute error increased for the separable task but remained constant for the integral task. As probability of the integral task increased, reaction time dropped abruptly for the integral task but elevated slowly for the separable task.

None of these cited research efforts focused on variation of operator knowledge, complexity of task, or comparison group performance in those tasks.

\subsection{Experiment Design}

It was first necessary to determine how many operator groups would be necessary, as well as how many scenarios would be run in order to distinguish significant differences between the groups. Details on this process are available in the following chapter (Experiment Design). The results dictated that 15 scenarios would be run between 4 groups (two expert and two standard groups for a balanced design with replicates). The study necessitates collecting continuous data (time to resolve), binomial data (resolved/not resolved), and subjective data using the Subjective Workload Assessment Technique (SWAT).

\subsection{Resources}

An assessment of the required resources was made. This project was extremely difficult in terms of the extensive coordination required between multiple agencies and the scheduling difficulties involved in working with an operational space squadron. To intensify the problem, numbers of available subjects able to participate due to job constraints were limited. However, these challenges merely required more development and coordination time to overcome.

The following resources were divided into two categories: 
4.3.1 Personnel. A total of ten participants (volunteers) were required to construct the following groups:

- Two Standard Crews - Eight 1 SOPS operators of the proper job specialty and experience level. Each standard crew of four requires two Satellite System Operators (SSO), one Ground Systems Operator (GSO), and one DSP Vehicle Engineer (ENG).

- Two Expert Crews - Each of the two expert crews will consist of one highknowledge level operator who will perform all satellite functions (SSO, GSO, and ENG).

\subsubsection{Equipment.}

- The appropriate DSP simulator for simulation purposes. This simulator should ideally support real-time anomaly injection, should not be code-intensive, and should be flexible enough to adjust to the dynamics of these unique scenarios. Most importantly, it should be capable of emulating the current ground system equipment.

- A DSP, Phase III vehicle database loaded for simulation purposes.

- Approval by 1 SOPS to use the above resources to include scenario execution and a brief training session for operator system usage.

Approval to use these resources had to be gained prior to further study development.

\subsection{Scenario Development}

After designing the experiment and determining the required resources, scenario development could begin. The spacecraft anomalies would be randomly injected into the scenarios, selecting one of each type (Type 1,2, or 3) per scenario. The anomaly bank was comprised of more than 100 possible satellite states, and the number of anomalies statistically required drove the number of scenarios needed. Simulation game rules, in addition to timekeeping/logging methods were also outlined. The following items were created for each scenario after the anomalies were randomly assigned: 
- A timeline for each scenario

- Scenario script

- Expected Courses of Action (COAs) to be taken for each anomaly, to include satellite signature, necessary commands, resulting satellite effects, and signatures of incorrect commanding

- Associated command plans for execution

- Satellite pass plans, technical documentation, and other materials typically required by the crews and engineers to perform their jobs

Scenario anomalies involved the three forms of malfunctions listed in the paragraphs below.

4.4.1 Vehicle Anomalies. The first step was to examine any available historical data for possible anomalies. This included a list of all potential anomalies and suspected causes. Extensive data was not readily available, so contractor personnel with system longevity were sought for additional information. Unfortunately, many anomalous conditions modeled in the study are rare, so roughly one year was spent analyzing vehicle technical publications and diagrams to anticipate the satellite signatures that would occur as a result of these anomalies. After a complete list was formed according to system publications and historical data, correlations were determined between anomalies and sub-system mnemonics (parameters). Roughly 1000 mnemonics (satellite measurands or parameters) exist for the vehicle, and 653 are currently displayed to the operators by the system. The remaining mnemonics compiled derived (non-displayed) parameters. 
4.4.2 Space Environment. Space environment can play a large role in satellite malfunctions. Unfortunately, it is difficult to attribute many failures to space environment, since how space environment affects vehicles is highly non-deterministic and still under scientific study. Although the design process takes into account modeling of these characteristics, it is difficult to fully simulate effects in a laboratory environment. Some major influences include solar wind/storms and geomagnetic changes and cycles. These phenomena manifest themselves in the form of telemetry bit-flips, activation of event alarms and similar effects.

\subsubsection{Electro-Magnetic Interference (EMI). Since Electro Magnetic Interference can be} instigated accidentally or intentionally, it was necessary to determine the frequency of both forms. Intentional EMI can be simple to detect, but very difficult to resolve or repair, and Space Control (denying enemy access to satellite assets) enters into the process when considering these cases. Unintentional EMI might typify a Type 1 anomaly, while intentional denial (Space Control) could be the equivalent of a Type 3 anomaly.

\subsection{Crew Selection}

As mentioned in section 4.3, a total of ten volunteers were required for the study. The crewmembers for the standard group were randomly sampled from the total available standard knowledge level operators available to participate in the $1^{\text {st }}$ Space Operations Squadron. A survey was conducted for crew personnel to determine average time spent performing the job on the current system. This equated to the mean experience level for the control group, which was approximately two year's experience on the system.

The same was done for the test (expert) group, where the experience level established for the expert group with an extensive satellite engineering background was 
approximately 7 years (with a range of 5 to 10 vears). For the expert group, there was an exceptionally small cadre of individuals who would be considered to fall into this category. Subsequently, it was impossible to sample randomly from such a small population; these members were merely selected.

Human Subject Protection concerns were also addressed. Coordination with the 1st Space Operations Squadron was designed to solicit volunteers for the standard crew force. There was no employment risk in project participation since the study had leadership support. The expert crew members were also volunteers, and they participated outside normal work hours. The crews had enthusiastic commitment and held a stake in the experiment results; they were aware that the outcome would affect future system design and subsequently their day-to-day operations. They were comprised of the best operators available and took the tasks seriously. The fact that they were volunteers demonstrated that they had dedication and made personal investment in the study.

Once the all participants were designated, they were provided with the SWAT card sort exercise. This exercise involved sorting through 27 cards and classifying these cards into perceived task difficulty categories. This method enables development of a scale based on the individual operators. The dynamic scaling enables quick collection of subjective data following the scenarios. More detail on the SWAT technique may be found in Chapter 5.

Each volunteer spent no more than 15 hours for simulations, and 4 hours in total to complete subjective task surveys. The crews were requested to attend a 30 -minute pre-brief/training session at study start and a 15-minute out-brief at the study conclusion. Subjective task surveys focused only on perceived task difficulty and task ratings; no further data was collected from these surveys. 
There was no race or sex criteria in subject selection. Furthermore, race or sex of study participants was not recorded or referenced in any way. Crew members were only designated according to group membership ("expert" or "standard"). The standard crews also have specialty positions designated, such as SSO, GSO, or ENG. Personal information other than group membership (expert or standard knowledge level) and approximate experience level/specialty was not used in this study. Extraneous information (name, sex of subject, etc.) has no contribution to the research and was not recorded or included in any associated study documentation.

Authorization from the Institutional Review Board may be found in Appendix B (Institutional Review Board Approval). The use of human subjects in this study has been approved by the University of New Hampshire Institutional Review Board for the Protection of Human Subjects in Research.

\subsection{Scenario Validation}

All scenarios were validated on the simulator prior to execution. This occurred over a two-week period in March 2001. Care was taken to ensure the scenarios functioned properly - minimal inputs were not easily replicated on the emulator. Manual inputs (real-time change of satellite mnemonics) were necessary for the items not automatically modeled by the emulator, while verbal inputs were needed for items the simulator was unable to replicate. These functions were identified well ahead of time so the scripts could be restructured and investigator tasks could be assigned to make presentation standardized and seamless. 


\subsection{Scenario Execution}

4.7.1 Timekeeping/Recording. As a backup method to manual timekeeping, each scenario was videotaped during execution so that time criteria could be re-validated and data could be accessed if necessary.

4.7.2 Randomization. Scenarios were run in a random order to minimize the potential for investigator learning, or improving scenario presentation due to repetition. The statistical software program, JMP, provided a random scenario scheme for all four crews. This scenario order is available in Chapter 5.

\subsubsection{Crew Alertness. Effort was also made to ensure all crews functioned at} approximately the same level of alertness. Ideally, the scenarios would be run for one crew each day, at the same time every day. However, resources in terms of the actual crewmember availability and emulator time did not make this a feasible option. Therefore, it was carefully ensured that within time-of-day constraints (morning or afternoon), the crews were similarly rested and alert. To guard against variation in alertness, scenario execution was restricted to the afternoon hours, ranging from 1200 to 1600.

4.7.4 Scenario Format. The scenarios were divided into three sections of pre-brief, scenario, out-brief/survey completion. Samples of the scenario flow to include the scenario script, briefings, and survey can be seen in Appendix D (Sample Scenario Format). In each situation, the starting status of the scenario was presented to the crews to include the current satellite status and the goals to be accomplished in the scenario. For all scenarios it was generally the same, with the exception of initial satellite state. Following the scenario, a short out-brief included thanking the crew and 
distributing the subjective surveys. For phase one of the survey, the crew was questioned as to what were the three anomalies. Following this phase, the correct three anomalies were confirmed to ensure they could complete the SWAT portion of the survey correctly.

4.7.5 Investigators. Two investigators collected the data and interfaced with the crew members. The primary investigator was the Ph.D. candidate, and the investigator assistant was a member of the Center for Research Support (CERES) at Schriever AFB, CO. Both investigators were operationally qualified in the case study system. They are also members of the Air Force Reserve component providing operational support to the 1 SOPS and have backgrounds in human machine interface design and human factors.

\subsection{Scenario Results and Data Analysis}

Upon collating the scenario results, the following data was interpreted:

4.8.1 Continuous Time and Binomial Data. Results may be found in the data presentation section (Chapter 6).

4.8.2 Anomaly Type Categorization. Although this will be discussed in more detail in the data presentation, generally the goal was to statistically determine what level of training and experience is required to identify and complete each Type $(1,2$, and 3$)$ of task(s) correctly and within time constraints. This data can aid in assessing anomaly difficulties. It can also illustrate what commanding functions lend themselves well to automation and which require extensive human knowledge and intuition. An associated goal is to formalize conclusions on the required knowledge level needed to perform certain operations. The findings can provide decision-makers with the ability to make 
knowledgeable choices on who should operate the system, at what level of expertise, and to identify the risk associated with the different choices of operator skill.

4.8.3 Subjective Measures. Subjective measures aid in evaluating anomaly difficulty, since time data is only one descriptor. Combining both data types create a more complete measurement of task difficulty. The Subjective Workload Assessment Technique (SWAT) data was collected via survey, and results may be found in the data presentation section.

\subsection{Current System Improvement}

Systems slated for improvement in terms of display formats and automation based on the scenario results are being tested at CERES. These systems are developed by applying Commercial Off-the-Shelf (COTS) ground system philosophies versus the specialized, costly equipment widely used for current military satellite command and control. The current system used in 1 SOPS is the same system emulated in this study by the Crew Training Emulator (CTE) simulators. Designed in the early 1970's, yearly cost sustainment now approaches $\$ 50$ million. Conversely, COTS systems adopt virtually "throw-away" technology, where the sustainment costs are covered by the vendor for the approximately three-year maintenance contract. After that period, the system is discarded and upgraded to the new capability. In this manner, customers/users are assured possession of the latest in technology and capability, while minimizing sustainment and maintenance costs.

The outcome of this research will drive the creation of new, enhanced, userfriendly and more informative HMI screens for an optimal user. In addition, the first part of the study will dictate what functions of satellite commanding should be automated, and which should be only minimally automated to ensure human intervention. Based on 
this project, it is the hope that future design of space systems will be driven by humanistic factors and not by machine hardware or software. 


\section{Chapter 5}

\section{EXPERIMENT DESIGN}

\subsection{Measuring Performance}

The following paragraphs detail the test process. In earlier chapters, there was brief mention of performance criteria. Accuracy, efficiency, and cost were noted as the discriminating parameters for gauging system success and operator performance. To explain further, accuracy translates into minimal number of errors, or success rate and efficiency equates to time required to resolve the anomaly. Financial cost contributes to determining which system can do the job the best for the least cost. Since one of the highest system expenses is attributed to operator costs, it follows that the expert operator system should be less costly to operate, given that it effectively provides the other two factors of efficiency and accuracy.

For example, a model outlined by Rohles (1988) describes performance and productivity. His model, derived from the learning theory of Clark Hull (1943) describes this relationship as:

$$
P=A * D+O
$$

where

$P=$ Performance

$A=$ Ability

$D=$ Drive

$O=$ Oscillatory factor, comprised of five factors: selection, training, supervision, communication, and environment. 
The "oscillatory" factor is a sizable but imprecise effect, and difficult to assess the most important contributor. Training is a tremendous issue in military satellite operations; most operators spend the majority of time on station in some form of training environment. Selection and environment encompass a wide spectrum of variables, such as human background, personality, and education level. It has been shown that education can positively affect performance levels (Huey, et al., 1992), even if it does not pertain to knowledge specific to the task. Better performance was associated with higher education (college degree versus high school) in an untrained system task randomly assigned across study participants.

\subsection{Experiment Description}

In this experiment, each crew (from knowledge groups "expert" and "standard") was subjected to a series of 15 scenarios containing three anomalies each, totaling 45 anomalies. Running four groups through each of the 15 scenarios resulted in 180 observations. The scenarios were one hour in length and of identical content among all crews. Anomalies were presented to the crew as the scenario began through errors displayed on the satellite telemetry frames. The crew teams tried to 1) identify, 2) prioritize, and 3) resolve/correct the anomalies within the scenario length. A time value was recorded upon resolution (or attempted resolution) of each anomaly. Those anomalies not resolved within the scenario time-span had a censored time value recorded equivalent to the difference between time at the end of the scenario and initiation time. The lead investigator who has 6 years of system experience determined the one hour time period. A typical, nominal satellite support lasts only 10 minutes, and would normally be extended in the event of an anomaly. Thirty minutes was then chosen as the scenario duration, but there was concern of this not being adequate time 
to address all three problems. Therefore, a sixty minute scenario was determined to be satisfactory for the crews to prioritize and resolve the anomalies.

\subsection{Assumptions and Constraints}

\subsubsection{General Assumptions and Constraints.}

- No changes in machine technology occurred in the study

- Two expert crews and two standard crews were tested to provide data replication and a balanced experiment design. Race or sex was not considered in crew selection

- An expert crew consists of one operator able to perform all satellite functions

- Selected expert operators have an average of seven years experience in DSP command, control and engineering of DSP systems

- Each standard crew consists of four operators, each individual possessing anywhere from six months to three years of operations experience on DSP systems

- The 15 scenarios are comprised of three satellite vehicle anomalies of increasing difficulty levels. Scenarios had one of each type 1, 2 and 3 anomaly difficulty levels that were presented simultaneously at the beginning of the scenario. Crews prioritized these as appropriate at the beginning of the satellite support.

- This project was completely dependent on using a simulator capable of emulating the DSP-I telemetry and commanding environment

- Data collection had to be completed prior to operational transition of the 1 SOPS. Test subjects for the standard knowledge base must be drawn from this operations center 
The only factor permitted to vary was operator knowledge level between the crews. This study could potentially be construed as a crew-reduction study, but it is not. Crew reduction may be an experimental result, but cannot be concluded without data analysis.

\subsubsection{Positional Aliasing. Positional aliasing describes a possible outcome where the} lower level knowledge crew could out-perform the expert crew merely by having more crew members. This implies that there could be aliasing of crew positions where there is perceived overlap between the engineering tasks and satellite operator tasks. After careful consideration, it was determined that aliasing would not be a factor. The primary reason is attributed to the operational structure imposed by the military for basic satellite operations. The only individuals who are authorized to access the keyboards and enter commands are the SSO position. Engineers, by job specialization, are not permitted to touch the operator consoles or perform commanding. Moreover, only one SSO is granted control of the contact, meaning one console is designated as the commanding console that can command the satellite. In addition, the SSOs have no exposure to any of the satellite technical documentation, and would therefore provide negligible aid to the engineers in researching subsystem characteristics.

This format of designated operator roles, although lending itself well to this experiment in terms of minimizing other undesirable effects of aliasing, is not the most efficient to run satellite operations. As shown in the research of Urban et al. (1992), "specialized" (non-hierarchically structured) teams were in all cases, regardless of workload, at an advantage over non-specialized, hierarchical teams. Urban's research case study used five-member teams to achieve this conclusion. 


\subsubsection{Ground System Operator (GSO) Position. The GSO position required}

considerable examination, namely on how team dynamics were influenced and what role they would have in the study. First, the GSO provided no satellite vehicle expertise to the crew. More important, since the ground segment functions nominally with no variation, there were essentially no inputs for the GSO. Initially, there was a desire to remove them from the crew dynamics altogether since there were concerns of this position becoming a distracter either due to member inactivity or individual boredom. However, there were two more pressing concerns: 1) the possible effect on crew dynamics, and 2) alteration of the standard crew structure and generalization of the study results of a crew of three versus a typical crew of four. The compromise reached was that the GSO would be present during the typical contact portion, or SOH phase. During this phase of the scenarios, each anomaly is ascertained to be either vehicle related or ground related (ground segment does not vary). After the GSO completes his function(s), he may depart the study environment. In this way, the crew integrity is maintained, but any opportunity for the GSO to be a crew detriment is minimized. The GSO provided no subjective or continuous data for use in the experiment, and did not participate in the closing subjective surveys.

\subsection{Research Questions}

The questions the study attempted to address are:

1. Can satellite anomalies be effectively categorized into three distinct types (according to difficulty) so that they can be used to guide automation of future space systems?

2. Is the difference in anomaly resolution accuracy between the two knowledge levels statistically significant? Accuracy refers to anomalies that were correctly 
resolved. Only anomalies solved accurately were considered "resolved" and given a resolution time.

3. Is the difference in anomaly resolution efficiency between the two knowledge levels statistically significant? Efficiency is defined by resolution time.

4. Are there differences at the $95 \%$ confidence level among the six treatments (specific main effect combinations, anomaly type by knowledge level interactions)?

5. Are there differential effects of knowledge level across the anomaly types on error resolution in both accuracy and efficiency?

6. Does higher knowledge level result in:

- a more accurate system based on fewer errors?

- a more efficient system based on less time required to resolve anomalies?

- a lower cost system based on fewer operators required?

\subsection{Factors}

Variables completely controlled by the experimenter are factors. In this experiment, there are two factors. One factor is knowledge type, which has two levels: high knowledge and low knowledge. The other factor is type of anomaly, having 3 levels: Type 1 (easy to resolve), Type 2 (intermediate difficulty), and Type 3 (difficult). Combining these two factors leads to a 2-by-3 factorial experiment. With this form of experiment, the effects of combining the factor levels can be investigated, forming 
$2 \times 3=6$ treatments. Treatments define specific combinations of factor levels.

\subsection{Raw data response variables}

Two response variables were measured. They are:

\section{Accuracy: Was the anomaly resolved?}

- Measurement of either 0 (not resolved) or 1 (resolved)

- This response has binomial characteristics

- Measuring the binomial response is commonly used to enable identification of censored (missing) observations

\section{Efficiency: Time to resolve the anomaly}

- Raw time values are measured as a continuous variable

- Censoring occurs if the anomaly is not resolved during the scenario

- Cox's Proportional Hazards Models were used to analyze the data. This technique is commonly used to analyze censored (missing) data

To further explain the two responses, the response variables were measured according to the team either resolving the anomaly (success $=1$ ) or not resolving the anomaly (failure $=0$ ) within the 60-minute satellite support. This was the binomial response of the anomaly being either resolved or not resolved. As it turns out, due to the fact that $97 \%$ of anomalies were resolved, the binomial data was not very useful other than to provide information on censored responses that could be used in continuous time data analysis.

Continuous time data provided the most information on each crew's ability to resolve anomalies. Time to resolve was recorded for each of the three anomaly types during the scenarios. If a particular anomaly was not resolved, a censored time value of 
the difference between scenario termination time and initiation time was recorded for the continuous response variable of time. This potential for occurrence of censored data leads to the use of Survival Analysis techniques. Cox's Proportional Hazards models were specifically applied since they account for censored observations, and model assumptions were satisfied. Cox's models use the censored data in an unbiased manner so that information is not lost in the analysis. Model specifics will be discussed in section 5.10.3.

A single scenario consisted of three anomalies, one of each difficulty type. All three anomalies were presented simultaneously upon scenario initiation. Essentially, there were three time measurements per scenario (representing the three anomalies), but occasionally these consisted of a mixture of measured and censored times. The process by which the team conducted anomaly resolution originated from the priority it assigned to each task; this is a realistic approach, since that is how crews conduct troubleshooting in practice. Therefore, the time recorded for each anomaly was dependent on the time the crew dedicated to resolving each of the three before 1) they solved the problem, 2) they gave up (resulting in censored time), or 3) the scenario ended (also resulting in censored time). There was no instance where the crew actually "gave up" - in all cases, the crews attempted resolution until the scenario time ended.

The scenario ground rules dictated notification by the crew lead of their intentions (priority and when they decided to give up and move on to the next anomaly), and this time was recorded exactly to second accuracy. The priority assigned to the anomalies did not necessarily mean that there was a set order in which the anomalies were resolved. However, more often than not, Type 1's were always assigned priority one. The process of priority assignment was a product of formal training and established military guidelines. 


\subsection{Sample Size Determination}

Two approaches were considered in sample size determination.

5.7.1 Binomial (measurement of "success" or "failure"). In the section below, the goal was to find the sample size required to estimate a treatment proportion with a bound on error of 0.1 . Here, it is assumed that the probability of successful anomaly resolution is $p=0.5$, giving the most conservative sample size.

The equation used solves for $n$, the required sample size for a predetermined $\varepsilon$, the maximum error of the point estimate $y / n$. For a $95 \%$ confidence level, the corresponding $Z$ value is 1.96 . Inserting the appropriate values, the sample size per treatment is determined as

$$
n=Z_{\alpha / 2}^{2} / 4 \varepsilon^{2}=(1.96)^{2} / 4(0.1)^{2}=96.04=97 \text { anomalies. }
$$

Recall that this is the required sample size per treatment for precise estimation of the given treatment proportion. Considerations of either two, three, or six treatments are of interest to compare the two knowledge levels, compare the three types of anomalies (which might include simply comparing Types 2 and 3), and compare all six knowledge level-by-type combinations. These comparisons would require as many as 582 observations.

An interesting point is that there were only six censored observations overall (out of 180 total observations), making a final binomial analysis not as useful as initially expected. However, binomial considerations were helpful during the initial experiment development phase in terms of exploring necessary sample size and expected resource needs. 


\subsubsection{Continuous time data.}

5.7.2.1 Methodology. The time to resolve was measured directly in simulator system time in seconds. As mentioned earlier, there were three time measurements per scenario (representing the three anomalies), but occasionally these consisted of a mixture of measured and censored times. Each typed command was displayed on the screen so that keystroke tracking was simple and exact. Times recorded were representative of the crews either resolving the problem or the scenario terminating (resulting in censored time). Times were manually recorded by two investigators and were backed up by video.

5.7.2.2 Sample size determination. The first step for sample size determination for the continuous time data is to estimate the standard deviation. This was based on the shortest and longest likely times required to resolve an anomaly. A typical, conservative estimate of standard deviation is range $\div 4$; a less conservative estimate is range $\div 6$. Both of these are based on properties of the normal distribution. Rather than predict the resolution range, the standard deviation was estimated as the scenario length (60 minutes) divided by the effective range of the standard normal distribution (6). The estimate of standard deviation for the experiment that was determined in this fashion was $\sigma \approx 10$ minutes (600 seconds). (As it turned out, this value of sigma was consistent with the root mean squared error (RMSE) of 620 seconds resulting from the actual analysis of variance performed for comparisons of type and knowledge.) Sample sizes were determined to ensure detection of a pre-specified difference of delta with probability $p=0.90$ when one is using an $\alpha=0.05$ level significance test. 
Results were recorded for a test set of continuous data for 2 and 6 groups in the statistical program JMP IN. It is important to note that for this type of analysis, JMP IN assumes equal stratum sizes. The Design of Experiments analysis tool was used to confirm hand-calculated sample size estimates. In the calculations, $\sigma$ and $\delta$ were set to 10 minutes and 7 minutes, respectively. These estimates were based on a power of $90 \%$ and the sigma and delta values defined above to find the number of anomalies required, or $\mathrm{n}$. The raw data is included in Appendix $A$, and the results are listed in the next section.

5.7.2.3 Sample size results. After examining the binomial and continuous data results, it seemed prudent in terms of resources and time considerations (to minimize operational impact to the squadron) to follow the values outlined in the continuous approach for sample size as opposed to the binomial trials. The binomial calculations, as expected, yielded large sample sizes (the 97 samples calculated were per treatment). However, the values obtained through JMP for the continuous data were much more reasonable. The conclusion is that for a significance level of $\alpha=0.05$, a power of $90 \%$, and accompanying sigmas and deltas of 10 and 7 , respectively, comparing the two knowledge-levels requires roughly 86 anomalies total, or 43 per group. This translates into almost 15 scenarios with 3 anomalies contained in each scenario. By increasing the delta to 10, the number of anomalies required per group dropped to 44 , or 22 observations per group. These calculations were performed using the equation (which is only applicable in a two-sample situation) (Ostle, et al., 1988):

$$
n=\frac{2\left[Z_{\alpha / 2}^{\cdot}+Z_{B}^{*}\right]^{2} \sigma^{2}}{\delta^{2}}
$$


where $Z_{\alpha / 2}^{\circ}$ and $Z_{\beta}^{*}$ are the respective areas of $\alpha / 2$ (1.960) and $\beta$ (1.282) from the normal tables student's t-test tables. Calculations were also confirmed using tables produced by Davies (1956), where the difference, $D=\delta / \sigma$ is calculated and matched to the level of t-test to determine approximate sample sizes based on a specified power and significance level. These sample size calculations were also verified using JMP, again showing that $n \approx 88$, or $n=44$ anomalies per group were necessary to detect a difference of $\delta=7$.

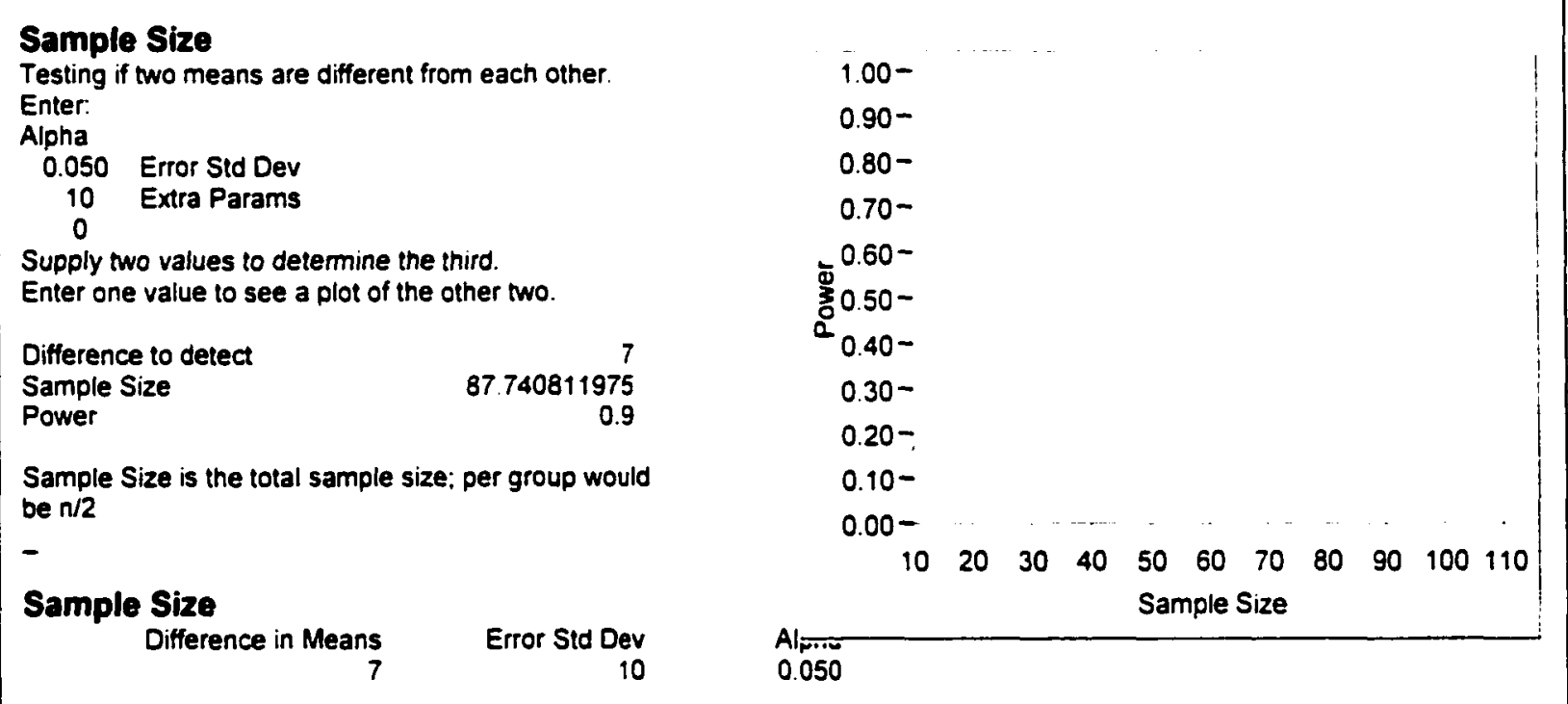

Figure 5.1: JMP Sample Size Determination for Comparison of Two Treatments

For computing required sample sizes for 6 treatments, the method is more complex. An iterative approach can be used (Montgomery, 1991; Scheffe, 1959) that approximates sample size per cell (or group).

$$
\Phi^{2}=\left[\delta^{2} /\left(2 a D^{2}\right)\right] n
$$


The term $D$ represents the maximum difference one wishes to detect. Using the equation above with the same value of sigma and selecting 7 units as the maximum difference $(D)$ to detect, the cell sizes were determined for a conservative size for $n$. Pearson and Hartley charts were then used to determine that a sample size of roughly 70 per group (420 total) would result in a power of approximately 0.925 .

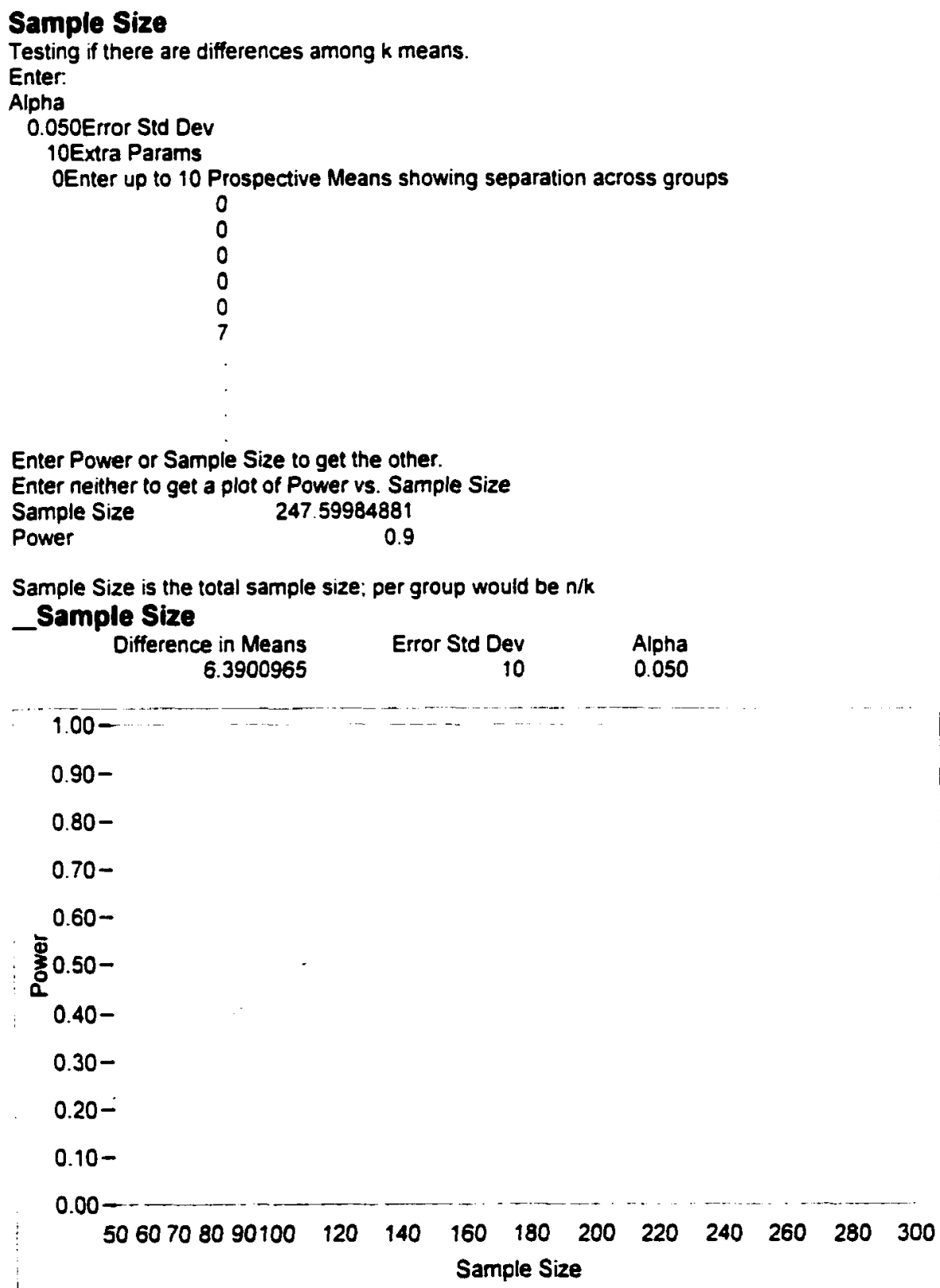

Figure 5.2: JMP Sample Size Determination for Comparison of Six Treatments 
Through JMP, it was also possible to verify the calculations for sample sizes necessary to determine differences in six groups for the six different treatment cases (Figure 5.2). For an $\alpha$ of 0.05 , the number of samples required for $\sigma=10, D=7$ were 248 observations total, or 42 per group. Figure 5.3 shows the comparisons of these results.

\begin{tabular}{|c|c|c|}
\hline \multicolumn{3}{|c|}{$\begin{array}{c}\text { Sample Size Determination Comparison for Binomial and Continuous Data } \\
\text { (Number of Anomalles Required) }\end{array}$} \\
\hline Treatments & Binomial Sample Size & $\begin{array}{c}\text { Continuous Sample Size } \\
(\alpha=0.05, \delta \text { (or } D)=7, \sigma=10)\end{array}$ \\
\hline 2 & 194 (97 per group) & 88 (44 per group) \\
\hline 3 & 291 & $\begin{array}{l}\text { - JMP gives } 120,40 \text { per group. } \\
\text { - Not calculated }(44<n<82)\end{array}$ \\
\hline 6 & 582 & $\begin{array}{l}\text { - JMP gives } 248,42 \text { per group. } \\
\text { - Calculations give } 490 \text { (82 per group) }\end{array}$ \\
\hline
\end{tabular}

Figure 5.3: Sample Size Determination Comparison for Binomial and Continuous Data

One problem encountered was the ability to code this many scenarios and have the crews execute them; the resources required would be too taxing on the operations squadron. The final decision was to use 15 scenarios, containing 3 anomalies per scenario to be executed by 4 crews. This resulted in 45 observations per group, or 180 observations total. This appeared adequate to detect differences between the two groups (minimum $n$ required was 44 per group), and most likely between the six groups (depending on the actual standard deviation). Therefore, the experiment schedule was adjusted to construct 15 scenarios, which was more reasonable for manpower support requests and time dedicated to scenario construction. Each scenario required a 
minimum of one month for development ( 1.5 years for all 15$)$, and the scenario validation period for all simulations was two months.

\subsection{Subjective Measures}

Subjective data was also collected from the participants. The technique selected was the Subiective Workload Assessment Technique (SWAT), which was developed by Brooks AFB Aeromedical Research Center. This method was selected from among those similar techniques, to include Modified Cooper-Harper, NASA-TLX. Paired Comparison Method for Interval Scaling (Turner, 1996) and the SWORD method. Most of these techniques in the past have been heavily applied to military operational task assessment - most often through aircraft pilot studies. All would have been suited to some degree to this study since satellite operations and aircraft operations have virtually identical characteristics.

Rueb, et al. (1994) used both the SWAT and SWORD techniques to assess aircrew subjective performance in a crew reduction application. SWORD was used in Rueb's study to further decompose the tasks. They praised the effectiveness of both techniques; however, the SWORD application was deemed unnecessary in this experiment. For this research, the additional set of measurands was not needed, as only the task of the anomaly resolution was of interest and not the separate actions that comprised the process. Hammer, et al. (1996) also used the SWAT method since it was designed by the Armstrong Aerospace Medical Research Laboratory to obtain subjective data from pilots in aircraft experiments. Consequently, it lent itself to this type of study also since "flying" satellites is acknowledged as being similar as flying aircraft.

Hendy, Hamilton, and Landry (1993) tested comparative effectiveness of the NASA-TLX and SWAT rating scales. Both techniques are multi-dimensional methods, and both exhibit similar characteristics and behavior. The methods incorporate scaling 
procedures for combining the results of a number of individual factor judgements into a single (scalar) index of overall workload. One drawback to SWAT is that it has slightly less sensitivity than TLX, which was also confirmed by Battiste and Bortulussi's (1988) research. However, SWAT requires less participant effort in completing surveys since the scaling is performed prior to data collection, an advantage when resources are only available for minimal time.

SWAT, in essence, provides a measure of subjective workload, which is defined as the orthogonal dimensions of time load $(T)$, mental effort load (E), and psychological stress load (S). The two phases of SWAT development are the scale development phase and the event scoring phase. The SWAT method requires an initial sort of 27 cards to be performed by the scenario participants. This card sort is used to develop the scale used to rate the subjective measures collected upon completion of the scenarios. These scaling factors are translated into interval scales for each participant to define a single metric of workload, and are developed prior to data collection. The scale used can be a group scale assuming that the entire subject pool has a high level of agreement in perceived difficulty elements (Kendall's Coefficient of Concordance $>0.75$ ). Using one scale for the entire group simplifies analysis considerably; however, individual scales can be developed for each subject if necessary. This scale rescales difficulty levels of events into a single numbers ranging from one to 100 . Following scale development, the event data can be collected from the participant rating satellite anomalies in terms of the three dimensions defined above. More specifically, each of the three anomalies in every scenario has three ratings: levels 1,2, or 3 in terms of time load $(T)$, mental effort load (E), and psychological stress load (S). This data is then analyzed according to the interval scale of workload units and anomaly difficulty user ratings. 


\subsection{Study Design}

The following section outlines specifics of study design, from the general process to particular details unique to the project.

5.9.1 Process. The following section details the step-by-step approach taken to the experiment.

1. Determine and list all possible anomalies under consideration. This was performed with the help of TRW, AEROJET, and Air Force personnel.

2. Group the anomalies into "Type" groups. Although this will be performed "a priori" as part of the study, this step is necessary in order to ensure anomaly complexity is evenly distributed throughout the scenarios.

3. Sample each of the "Type" groups to select the anomalies for injection into the scenarios. Create scenarios out of the randomly selected anomalies of the total $\mathbf{N}$ anomalies grouped into 3 type groups. Each scenario should contain $\boldsymbol{R}$ (selected as 3) anomalies per scenario. Random selection of anomalies was performed using the JMP statistics software "shuffle" option.

4. Create $\mathbf{S}$ (selected as 15 ) scenarios.

5. Total number of scenarios executed equaled $X(15$ scenarios $* 4$ groups $=60)$ trials for both groups A (expert knowledge level) and B (standard knowledge level). The same scenarios must be run for both groups in random order, and scenarios were randomized using the JMP software.

6. Identified signatures for each anomaly included in the scenario: how the anomaly is represented to operators on the telemetry screens. This was the most time consuming portion of the experiment, as in-depth satellite engineering knowledge was required to accomplish scenario design. Development involved use of vehicle 
wiring diagrams to anticipate how the signature would appear and what command sequences were necessary to remedy the problems.

7. Construct the simulations and code them into the simulator. This was performed on the crew Training Emulators designed to simulate the existing 1 SOPS operational system.

8. Test and verify all scenarios.

9. Execute the same scenarios for groups $A$ and $B$. The $X$ scenarios should have elements of Type I, II, and III anomalies. Group performance was manually recorded during each scenario, using video back up. Manual recording was accomplished via the emulator time-stamp, where each command is denoted on the computer screen according to time transmitted.

\begin{tabular}{|c|c|c|c|c|}
\hline & & CRE & & \\
\hline & Standard-1 & Standard-2 & Expert-1 & Expert-2 \\
\hline & 9 & 2 & 15 & 4 \\
\hline & 6 & 10 & 7 & 12 \\
\hline & 13 & 15 & 12 & 5 \\
\hline$y$ & 11 & 8 & 3 & 9 \\
\hline 8 & 3 & 5 & 13 & 15 \\
\hline בี้ & 8 & 3 & 10 & 13 \\
\hline o & 10 & 7 & 11 & 1 \\
\hline$\xi$ & 4 & 14 & 14 & 6 \\
\hline 寻 & 15 & 12 & 2 & 3 \\
\hline ๑ & 2 & 4 & 8 & $\begin{array}{l}2 \\
7\end{array}$ \\
\hline & $\begin{array}{l}1 \\
7\end{array}$ & $\begin{array}{r}13 \\
1\end{array}$ & $\begin{array}{l}4 \\
1\end{array}$ & $\begin{array}{r}7 \\
11\end{array}$ \\
\hline & 12 & 6 & 6 & 10 \\
\hline & 14 & 11 & 9 & 8 \\
\hline & 5 & 9 & 5 & 14 \\
\hline
\end{tabular}

Figure 5.4: Randomized scenario order 
10. Collect Subjective Data following each scenario using the designed surveys that support the SWAT technique.

11. Analyze the time data and perform 'a priori' type classification. Time data was analyzed using Cox's Proportional Hazards model. Although type categorization was performed initially for the anomaly sampling and scenario construction, this will be performed again using the data obtained (time to resolve and perceived difficulty) to ensure proper categorization.

5.9.2 Anomaly sampling techniques. The sampling scheme used was obtained using the JMP software "shuffle" function to ensure randomization (Figure 5.4). The anomalies were initially sampled from the bank of all anomalies so that 15 of each type were selected, and then were combined to form the scenarios. There were over 100 anomalies available for sampling. For each scenario, one of each type of anomaly was chosen at random so that all scenarios incorporated 3 anomalies.

\subsubsection{Crew sampling techniques and associated effects.}

5.9.3.1 Sampling and selection. A tabular representation can be viewed in Figure 5.5. Each standard crew is comprised of four members, and an operator is defined by a single crewmember (four operators per crew). These individuals will accomplish two things: 1) collectively interpret identical computer displays representing the same anomalies, and 2) simultaneously attempt to resolve them as a team. This team concept and variation of knowledge within those teams contribute to nesting of the crews within knowledge levels (this effect will be discussed in section 5.9.3.2).

The crew members were randomly selected from available volunteers. With random selection, the variation that is contributed by different crews can also be 
estimated (the random effect of "crew"). The random crew selection process was limited in two ways: 1) the crews must contain all four crew positions, and these individuals must be selected among those members qualified in that crew position, and 2) selections must be made from those members who choose to participate in the study (which did not include the total operational crew force).

One substantial issue was that the number of expert operators is alarmingly small, and the sample size in which to draw capable, experienced subjects was miniscule. Qualified subject limitations have promoted use of such methods by promising appropriately powerful statistical comparisons of many conditions using few subjects (Bramwell, Bittner, and Morrisey, 1992). Limited "testbed" availability also has

\begin{tabular}{|c|c|c|c|c|c|}
\hline & & \multicolumn{4}{|c|}{ Knowledge Groups } \\
\hline \multirow{5}{*}{$\begin{array}{l}\text { Scenario } 1 \\
\text { (Block 1) }\end{array}$} & Anomaly & \multicolumn{2}{|c|}{ Expert } & \multicolumn{2}{|c|}{ Standard } \\
\hline & & Crew 1 & Crew 2 & Crew 1 & Crew 2 \\
\hline & 1-Low & & & & \\
\hline & 2-Med & & & & \\
\hline & 3-High & & & & \\
\hline \multirow[t]{5}{*}{$\begin{array}{l}\text { Scenario } 2 \\
\text { (Block 2) }\end{array}$} & $\begin{array}{c}\text { Anomaly } \\
\text { Type Difficulty }\end{array}$ & \multicolumn{2}{|c|}{ Expert } & \multicolumn{2}{|c|}{ Standard } \\
\hline & & Crew 1 & Crew 2 & Crew 1 & Crew 2 \\
\hline & 1-Low & & & & \\
\hline & 2-Med & & & & \\
\hline & 3-High & & & & \\
\hline$\cdots$ & $\cdots$ & \multicolumn{2}{|c|}{$\cdots$} & $\cdots$ & $\cdots$ \\
\hline $\begin{array}{c}\text { Scenario } 15 \\
\text { (Block 15) }\end{array}$ & $\begin{array}{c}\text { Anomaly } \\
\text { Type Difficulty }\end{array}$ & \multicolumn{2}{|c|}{ Expert } & \multicolumn{2}{|c|}{ Standard } \\
\hline & & Crew 1 & Crew 2 & Crew 1 & Crew 2 \\
\hline & 1-Low & & & & \\
\hline & 2-Med & & & & \\
\hline & 3-High & & & & \\
\hline
\end{tabular}

Figure 5.5: Experiment design 
promoted use of repeated measures as a means to maximize sensitivity within available time and funding resources (Bittner, 1992). Some of the most challenging repeated measures problems are Practice Effects (learning curve), Carry-Over and Sensitization Effects, and Situational Demand Characteristics (performance flexibility) (Weimer, 1995).

To familiarize the reader with the current experience level of the operations crews, the time on crew was recorded for each individual and position, and the mean was determined for all members who are currently certified in the squadron. Figure 5.6 provides a brief description of an average crew member at a particular snapshot in time. It is important to note that this parameter changes with time, so the composite crew average varied in appearance as the time to conduct the test approached. The data yielded the values shown in the table (as of 9 May 99). The crews selected to participate in the study had the experience composition shown in Figure 5.7.

\begin{tabular}{|l|c|}
\hline \multicolumn{1}{|c|}{ Position } & $\begin{array}{c}\text { Mean time qualified on crew } \\
\text { (days) }\end{array}$ \\
\hline Ground System Operator & 565 \\
\hline Satellite System Operator & 424 \\
\hline Crew Commander/ Satellite System Operator & 396 \\
\hline DSP Engineer & 252 \\
\hline
\end{tabular}

Figure 5.6: Crew qualification times (all operations crews, standard level) 


\begin{tabular}{|c|c|}
\hline CREW & Time qualified on crew (months) \\
\hline Standard - 1 & 10 \\
Ground System Operator & 25 \\
Satellite System Operator & 21 \\
Crew Commander & 34 \\
DSP Engineer & 24 \\
\hline Standard - 2 & 21 \\
Ground System Operator & 27 \\
Satellite System Operator & 20 \\
Crew Commander & 37 \\
DSP Engineer & \\
\hline Expert - 1 & 159 \\
Engineer/Operator (quad-qualified) & \\
\hline Expert - 2 & \\
Engineer/ Operator (quad-qualified) & \\
\hline
\end{tabular}

Figure 5.7: Subject crew qualification times

5.9.3.2 Nesting. Effects restricted to a single level of a factor are said to be nested within that factor. Nesting is often used as a method to reduce residual variation by allocating variation. In this study, the factor of crew is nested within knowledge level and will be treated as a random effect. The use of nesting is valuable in this case: because the response measured is a crew response, nesting allows estimation of the variation attributable to use of the various crews. The design factor of crew is nested within knowledge level and random since both crews within each factor level are considered a random selection from all possible crews. Figure 5.8 demonstrates how nesting results in the experiment. 


\begin{tabular}{|c|c|c|c|c|c|}
\hline & & \multicolumn{4}{|c|}{ Groups } \\
\hline \multirow{5}{*}{ 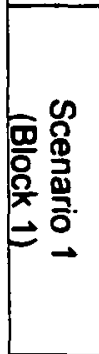 } & Types & \multicolumn{2}{|c|}{ Expert } & \multicolumn{2}{|c|}{ Standard } \\
\hline & Crew & $\begin{array}{c}\text { Crew of } 1 \\
\text { (trial 1) }\end{array}$ & $\begin{array}{l}\text { Crew of } 1 \\
\text { (trial 2) }\end{array}$ & $\begin{array}{c}\text { Crew } 1 \text { - Four Ops } \\
\text { (trial 3) }\end{array}$ & $\begin{array}{c}\text { Crew } 2 \text { - Four Ops } \\
\text { (trial 4) }\end{array}$ \\
\hline & 1 & & & & \\
\hline & 2 & & & & \\
\hline & 3 & & & & \\
\hline \multirow{5}{*}{ 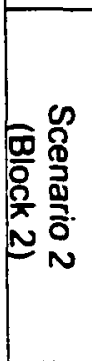 } & Types & \multicolumn{2}{|c|}{ Expert } & \multicolumn{2}{|c|}{ Standard } \\
\hline & Crew & $\begin{array}{c}\text { Crew of } 1 \\
\text { (trial 1) }\end{array}$ & $\begin{array}{l}\text { Crew of } 1 \\
\text { (trial 2) }\end{array}$ & $\begin{array}{c}\text { Crew } 1 \text { - Four Ops } \\
\text { (trial 3) }\end{array}$ & $\begin{array}{c}\text { Crew } 2 \text { - Four Ops } \\
\text { (trial 4) }\end{array}$ \\
\hline & 1 & & & & \\
\hline & 2 & & & & \\
\hline & 3 & & & & \\
\hline$\ldots$ & $\ldots$ & & $\ldots$ & $\ldots$ & $\ldots$ \\
\hline \multirow{5}{*}{ 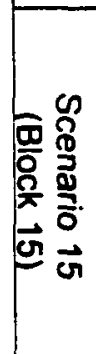 } & Types & \multicolumn{2}{|c|}{ Expert } & \multicolumn{2}{|c|}{ Standard } \\
\hline & Crew & $\begin{array}{c}\text { Crew of } 1 \\
\text { (trial } 1 \text { ) }\end{array}$ & $\begin{array}{l}\text { Crew of } 1 \\
\text { (trial 2) }\end{array}$ & $\begin{array}{c}\text { Crew } 1 \text { - Four Ops } \\
\text { (trial 3) }\end{array}$ & $\begin{array}{c}\text { Crew } 2 \text { - Four Ops } \\
\text { (trial } 4 \text { ) }\end{array}$ \\
\hline & 1 & & & & \\
\hline & 2 & & & & \\
\hline & 3 & & & & \\
\hline
\end{tabular}

Figure 5.8: Nesting/blocking scheme

5.9.3.3 Blocking. A block corresponds to trials of an experiment that occur under similar conditions. Simply stated, a block is a homogeneous grouping of experimental trials. Figure 5.8 shows applicable blocking schemes, and depicts running 15 scenarios total (based on resource limitations), having equal combinations of anomaly difficulties in the scenarios. To explain in more detail, Scenario 1 contains three anomalies that were presented to each of the four crews in random order. Since the anomalies were identical, the responses to Scenario 1 form a block. Therefore, 15 total blocks (scenarios) result. As noted in the table, each scenario will be run four times in random 
order, twice for experts and twice for standard crews, resulting in four replications of the experiment.

In this experiment, blocking on scenario allows estimation of scenario differences. This allows one to focus more sharply on knowledge level and type differences, and provides greater power to detect differences in the levels of these factors. Scenario variation is treated as nuisance variation; it is estimated and eliminated from further consideration.

\subsection{Analysis Methods}

The following section outlines the methods employed to analyze the raw time data.

5.10.1 Variability Charts. This technique was used to examine the integrity of the data, determine points that were outliers, and estimate the data distribution. During this portion, natural log transformations of the data were also examined. The JMP software was used to accomplish all of these functions, and the actual analysis may be viewed in the next chapter.

\subsubsection{Analysis of Variance (ANOVA). Since the Proportional Hazards platform} (discussed in the section 5.10.3) in JMP does not allow for examination of random effects, the ANOVA analysis was first used to see if random effects of crew could be eventually neglected. The ANOVA method was used to estimate the contribution of residual variation for the random effects of crew, and these estimates can be viewed in section 6.2.1. 


\subsubsection{Cox's Proportional Hazards Model (for Continuous Data). Cox's Proportional}

Hazard's model is used extensively in medical studies for survival data. The model provides the researcher with the ability to distinguish differences in hazard functions without specifying the form of the functions, producing an assessment of survival time unbiased by incomplete (censored) data. The Proportional Hazards model is a semiparametric technique that allows for comparisons between hazard and survival functions for multiple groups. The baseline hazard function is the non-parametric portion of the model, while the linear portion is parametric.

As mentioned, the model uses censored (missing) data and enables examination of the impact of several different variables between groups. In the experiment, censored data results when measurements of the continuous response of "time to resolve" exceed the scenario length, or the crew has conceded defeat by that anomaly. If the anomaly is not resolved, the time spent trying to resolve the anomaly will be measured as a censored observation, resulting in right-censored data. The appeal of this analysis method is that it uses censored data in a manner such that it is unbiased. For the purposes of this experiment, the traditional term "failure rate" describes the "success of anomaly resolution rate", and "time to event" is time to resolution or censoring (up to the 60 minute maximum).

There are two descriptions of time to an event: survival probability and hazard rate, and these are directly related. Survival time is synonymous with either failure time. time to an event, or duration. Survival data is usually best modeled by non-normal distributions, such as the exponential, Weibull, and lognormal. The hazard function can be defined as the instantaneous failure (or duration) rate, and the survivorship function is the probability that an individual survives longer than time, $t$.

Two significant properties that should be checked when considering this analysis method are that the survival functions do not cross (since the hazard functions are 
assumed to be proportional), and that the difference between $\log -\log$ transformations of the two survival functions associated with the two proportional hazard functions are constant (Selvin, 1995). When these assumptions are satisfied, one is able to apply Cox's Proportional Hazards model appropriately.

Recall that the hazard function can be defined as the instantaneous failure (or duration) rate, and the survival function is the probability that an individual survives longer than time, $t$. The survival function and hazard functions (Lee, 1992), respectively, can be written as

$$
\begin{aligned}
& S(t)=\text { Probability \{an individual survives longer than time, } t\}=P(T>t) \\
& \lambda(t)=\lim _{\Delta t \rightarrow 0} \frac{P\{\text { an individual of age t fails in the age interval }(t, t+\Delta t)\}}{\Delta t} .
\end{aligned}
$$

The relationship between the hazard function, $\lambda(t)$ and survivorship function, $S(t)$ at a specific time $t$ is

$$
\lambda(t)=-\frac{d S(t) / d t}{S(t)}
$$

If the concepts of hazard rates and survival functions are applied to the specific case of proportional hazards models, the general proportional hazards models for the $f^{\text {th }}$ hazard function and $k$ covariates can be written as

$$
\lambda_{j}\left(t ; x_{1 j}, x_{2 j}, \ldots, x_{k j}\right)=\lambda_{0}(t) c\left(x_{1 j}, x_{2 j}, \ldots, x_{k j}\right)=\lambda_{0}(t) e^{\left\{\sum_{i=1}^{k} b_{i} x_{i j}\right\}}
$$


Selvin (1995) discusses the proportionality property of survival curves and hazard functions related by a constant, $c$. This proportionality follows from the equation above, and can be described as:

$$
\lambda_{1}(t)=c \lambda_{2}(t) \text { (Hazard functions of two groups) }
$$

and

$$
S_{1}(t)=\left[S_{2}(t)\right]^{c} \text { (Survival functions of two groups) }
$$

where the constant of proportionality, $c=\lambda_{1}(t) / \lambda_{2}(t)$. $S_{1}(t)$ and $S_{2}(t)$ represent survival times for two groups of interest, 1 and 2 , and $\lambda_{1}(t), \lambda_{2}(t)$ are their associated baseline hazard functions. The proportionality of both the hazard functions (and consequently, the survival functions) follows from the initial assumption of proportional hazards introduced earlier.

Selvin (1991) states that the constant of proportionality, c, can also be factored into a series of relative hazard ratios for several $(k)$ independent variables, or

$$
\begin{gathered}
\lambda_{1}\left(t ; x_{11}, x_{21}, x_{31}, \ldots, x_{k 1}\right) / \lambda_{2}\left(t ; x_{12}, x_{22}, x_{32}, \ldots, x_{k 2}\right)=\hat{c}=c_{1} c_{2} c_{3} \ldots c_{k}, \\
\text { where } c_{j}=e^{b_{1}\left(x_{11}-x_{12}\right)} .
\end{gathered}
$$

Explained simply, if two hazard functions are proportional, the constant of proportionality factors into relative hazard ratios; and each constant, $c_{j}$, reffects the separate role of an independent variable. This enables the comparisons for two groups experiencing several different indicator variables, and comparing the composite effects of multiple covariates simultaneously. 
Covariates considered were knowledge level, anomaly type, scenario, crew (nested within knowledge level, random effect), and the interaction of type and knowledge (type * knowledge). The censoring indicator was either 0 (resolved) or 1 (unresolved, or censored).

The proportional hazards model for this specific experiment can be described as:

$$
\begin{aligned}
& \lambda_{j}(t)=\lambda_{0}(t) \exp \left\{b_{1} x_{\text {knowledge }}+b_{2} x_{\text {type(1) }}+b_{3} x_{\text {type }(2)}+\right. \\
& b_{4}\left(x_{\text {type(1) }} x_{\text {knowledge }}\right)+b_{5}\left(x_{\text {type(2) }} x_{\text {knowledge }}\right)+b_{6} x_{\text {[crew(exp)[knowledge] }}+ \\
& \left.b_{7} x_{\text {[crew(std)/knowledge] }}+\sum_{i=1}^{15} b_{i}^{\prime} x_{\text {scenario_i }}\right\}
\end{aligned}
$$

where $\lambda_{j}(t)$ represents the $j^{\text {th }}$ hazard function (maybe say "represents the hazard function for the jth treatment") and $\lambda_{0}(t)$ is the baseline hazard function.

One null hypothesis of interest is the null hypothesis $\left(H_{0}\right)$ that there is no difference between the two knowledge group anomaly resolution times, versus the alternative hypothesis $\left(H_{\mathbf{a}}\right)$ that a difference does exist. Mathematically, these can be written as:

$$
\begin{aligned}
& H_{0}: \hat{c}=1 \text {, or } \lambda_{1}\left(t ; x_{\text {knowledge }(\text { exp })}\right)=\lambda_{2}\left(t ; x_{\text {knowledge }(s t d)}\right) \\
& H_{\mathrm{a}}: \hat{c} \neq 1 \text {, or } \lambda_{1}\left(t \left(t ; x_{\text {knowledge }(e x p))} \neq \lambda_{2}\left(t ; x_{\text {knowledge }(s t d)}\right) .\right.\right.
\end{aligned}
$$

This hypothesis was tested for the two knowledge groups of expert and standard, where knowledge was used as the grouping variable.

A different null hypothesis is that there is no difference in anomaly difficulty, versus the alternative that a difference exists. This hypothesis is: 


$$
\begin{aligned}
& \mathrm{H}_{0}: \hat{c}=1, \text { or } \lambda_{1}\left(t ; x_{\text {type }(1)}\right)=\lambda_{2}\left(t ; x_{\text {type(2) }}\right)=\lambda_{3}\left(t ; x_{\text {type(3) }}\right) \\
& H_{\mathrm{a}}: \hat{c} \neq 1, \text { or } \lambda_{1}\left(t ; x_{\text {type(1) }}\right) \neq \lambda_{2}\left(t ; x_{\text {type(2) }}\right) \neq \lambda_{3}\left(t ; x_{\text {type }(3)}\right) .
\end{aligned}
$$

The professional edition of JMP has Cox's Proportional Hazards model as an analysis tool option, and this platform was used for data analysis. This model in JMP supports nesting, but not random effects.

\subsection{Summary}

The intent of this chapter is to outline the plan to answer the research questions posed in Section 5.4:

1. Can satellite anomalies be effectively categorized into 3 distinct types (defining knowledge level required for resolution success) so that they can be used to guide automation of future systems? The SWAT technique was used to address this question.

2. Is the difference in terms of anomaly resolution accuracy between two knowledge levels statistically significant? Resolution accuracy resulted in a measurable, uncensored resolution time for analysis. Indirectly, Cox' Proportional Hazards models were used to address this question.

3. Is the difference in terms of anomaly resolution efficiency between two knowledge levels statistically significant? Cox' Proportional Hazards models were used to address this question.

4. Are there differences at the $95 \%$ confidence level among the six treatments (specific main effect combinations, anomaly type by knowledge level 
interactions)? Cox' Proportional Hazards models were used to address this question.

5. Are there differential effects of knowledge level across the anomaly types on error resolution time (accuracy and efficiency)? Cox' Proportional Hazards models were used to address this question.

6. Does higher knowledge level result in:

- a more accurate system (fewer errors)?

- a more efficient system (less time required to resolve anomalies)?

- a lower cost system (fewer operators required)?

The belief is that these questions can be answered satisfactorily using the statistical techniques described in this chapter. The combinations of objective and subjective data should provide an accurate and comprehensive view of the operator environment and task perceptions, as well as the variation in performance between groups of varying experience and knowledge. 


\section{Chapter 6}

\section{DATA PRESENTATION AND RESULTS}

\subsection{Introduction}

The software packages used for data analysis were the JMP Professional and JMP student versions created by the SAS Institute. Subjective data was analyzed using the SWAT Version 3.1, released by Gary Reid of the Armstrong Aerospace Medical Research Laboratory.

\subsection{Time Data}

6.2.1 Initial Analysis. A first look of the raw resolution time data yielded descriptive statistics of a mean of 494 seconds (approximately 8 minutes) and a standard deviation of 620 seconds (approximately 10 minutes). A complete table of means and standard deviations for each crew (excluding the censored observations) may be viewed in Figure 6.1. Note that the sigmas are relatively narrow and the means increase, as expected, with complexity. However, the Type 1 mean value for the Standard-2 Crew does not follow the other Type 1 mean trends -- the value of 393 seconds seems significantly larger than the other Type 1 means. This is due to two large resolution times for this crew (Type 1 anomaly resolution times of 1103 and 1303 seconds). If these two "outliers" are excluded, the mean Type 1 resolution time for the Standard-2 Crew, drops to 268 seconds. 


\begin{tabular}{|c|c|c|c|c|c|}
\hline \multicolumn{6}{|c|}{ Crew } \\
\hline$\frac{8}{8}$ & 3 & $\begin{array}{l}\text { Standard-1 } \\
\mu=168.270 \\
\sigma=122.601 \\
\mu=433.857 \\
\sigma=126.904 \\
\mu=707.154 \\
\sigma=131.694\end{array}$ & $\begin{array}{l}\text { Standard-2 } \\
\mu=392.667 \\
\sigma=114.713 \\
\mu=366.929 \\
\sigma=118.739 \\
\mu=811.929 \\
\sigma=118.739\end{array}$ & $\begin{array}{r}\text { Expert-1 } \\
\mu=177.071 \\
\sigma=127.018 \\
\mu=405.600 \\
\sigma=122.711 \\
\mu=765.467 \\
\sigma=122.711\end{array}$ & $\begin{aligned} & \text { Expert-2 } \\
\mu= & 153.200 \\
\sigma= & 51.232 \\
\mu & =215.867 \\
\sigma & =51.232 \\
\mu & =322.467 \\
\sigma & =51.232\end{aligned}$ \\
\hline
\end{tabular}

Figure 6.1: Means and standard deviations for resolution times (six censored observations excluded)

Upon initial inspection of the data, it was apparent that the resolution time data (in seconds) was not normally distributed. The natural log was taken of all resolution time measurements, and a Shapiro-Wilk test for normality was conducted (the test hypothesizes that the data is normally distributed). Test results showed that although the untransformed data was not normal (Shapiro-Wilk p-value $\approx 0$ ), the log-transformed data did not show evidence of non-normality ((Shapiro-Wilk $p$-value $=0.1767)$. Plots and normality tests are shown in Figure 6.2.

The raw resolution time measurements most closely followed a lognormal distribution (a distribution of a variable whose logarithm follows the normal distribution). The Weibull distribution was also a close candidate. However, the lognormal proved to provide a better fit. Therefore, there is strong evidence that the In(time) data is normal since both the lognormal fit the raw data well and the log transformed data passed the Shapiro-Wilk test. The lognormal fit and parameter estimates are shown in Figure 6.3; censored times are boxed in red. 


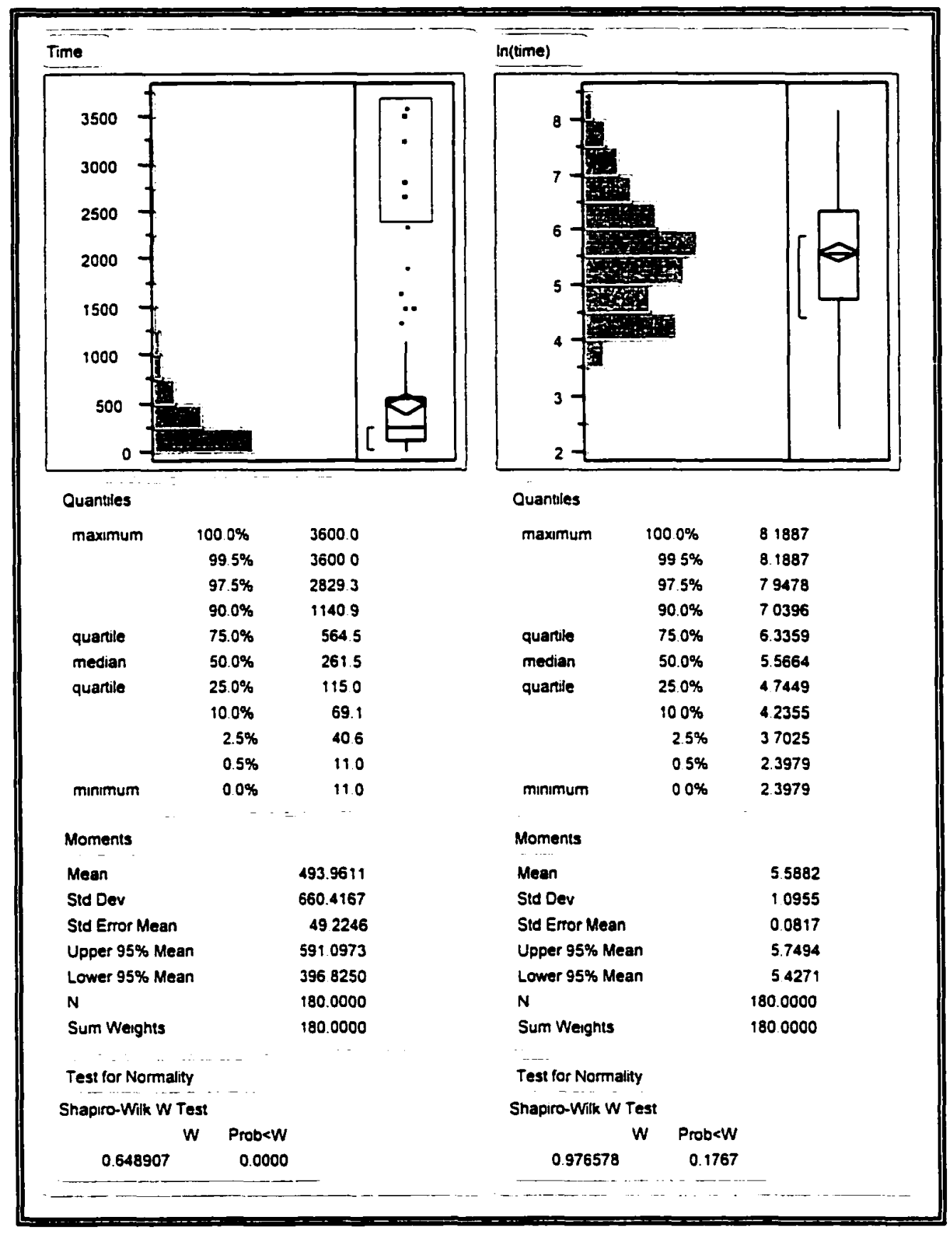

Figure 6.2: Distribution Analysis (time and In(time)) 


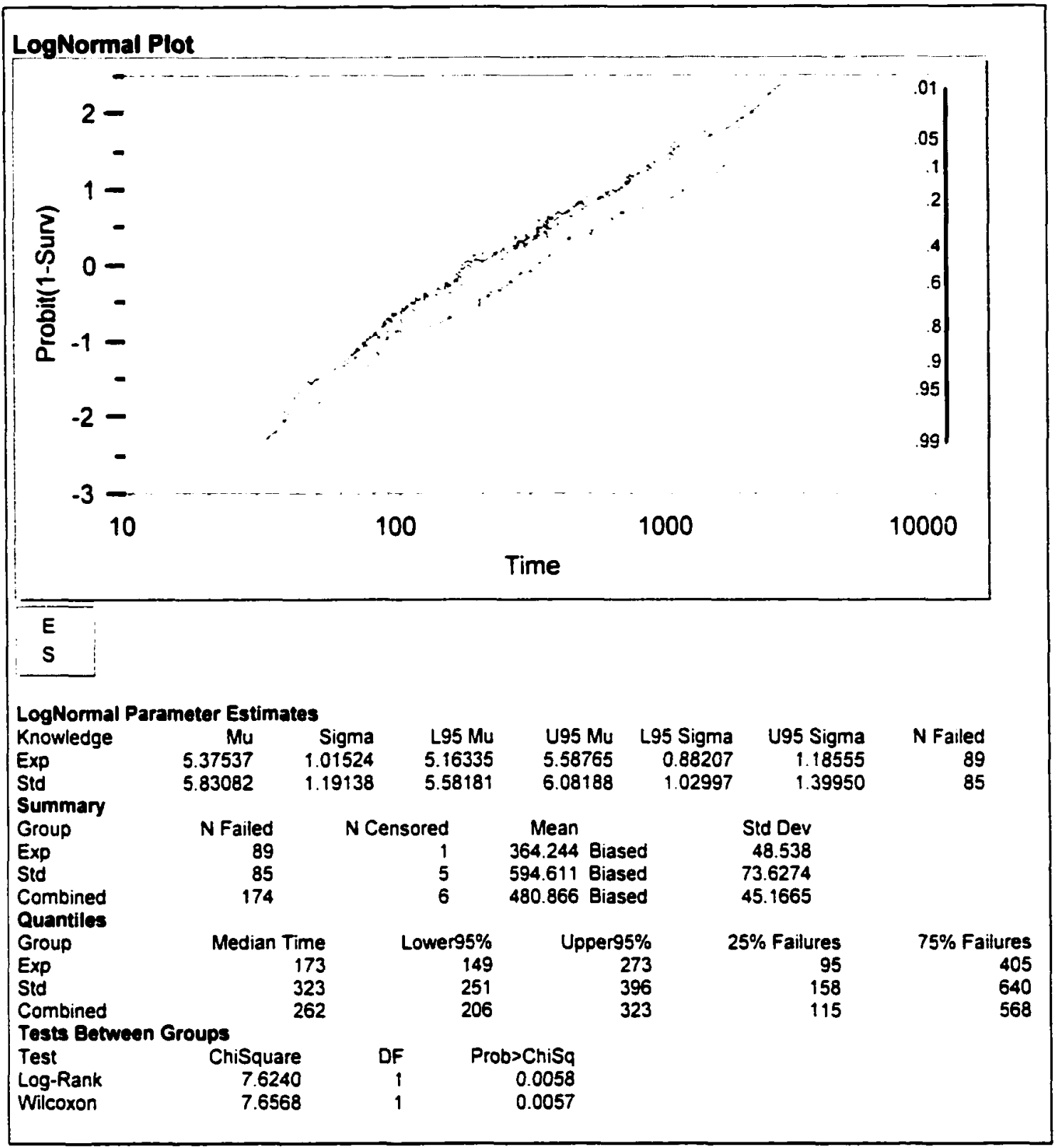

Figure 6.3: Lognormal fit for both knowledge levels

Variability plots depict the variation of the time data across scenario, knowledge group, and anomaly type. Large colored blocks denote censored observations in the time graphs only. The first set of plots (time) on each variability chart represent the raw (untransformed) data. These plots are presented in Figures 6.4-6.7. 


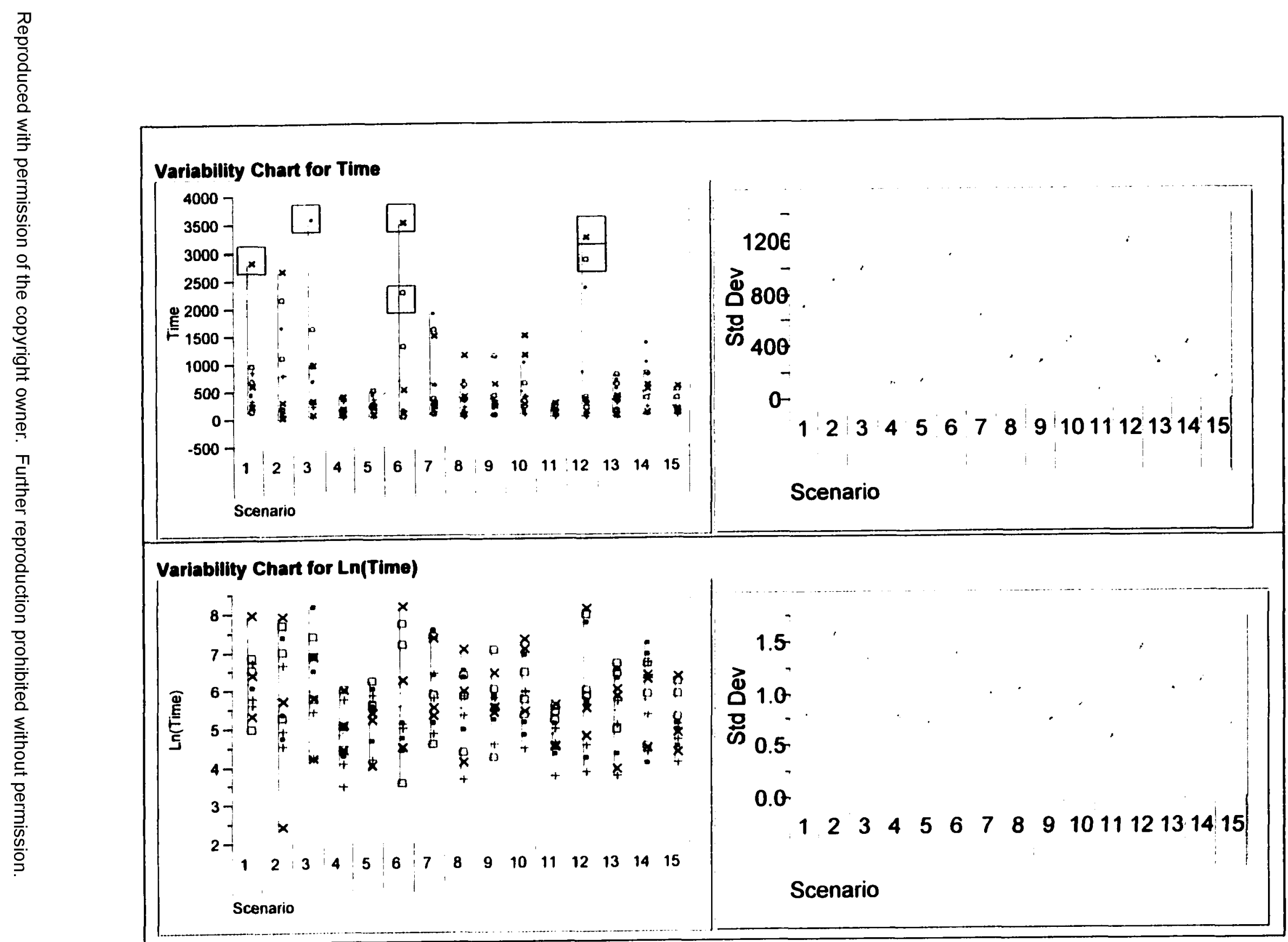

Figure 6.4: Variability charts for scenario vs. time and In(time). Symbols represent individual crew observations. 


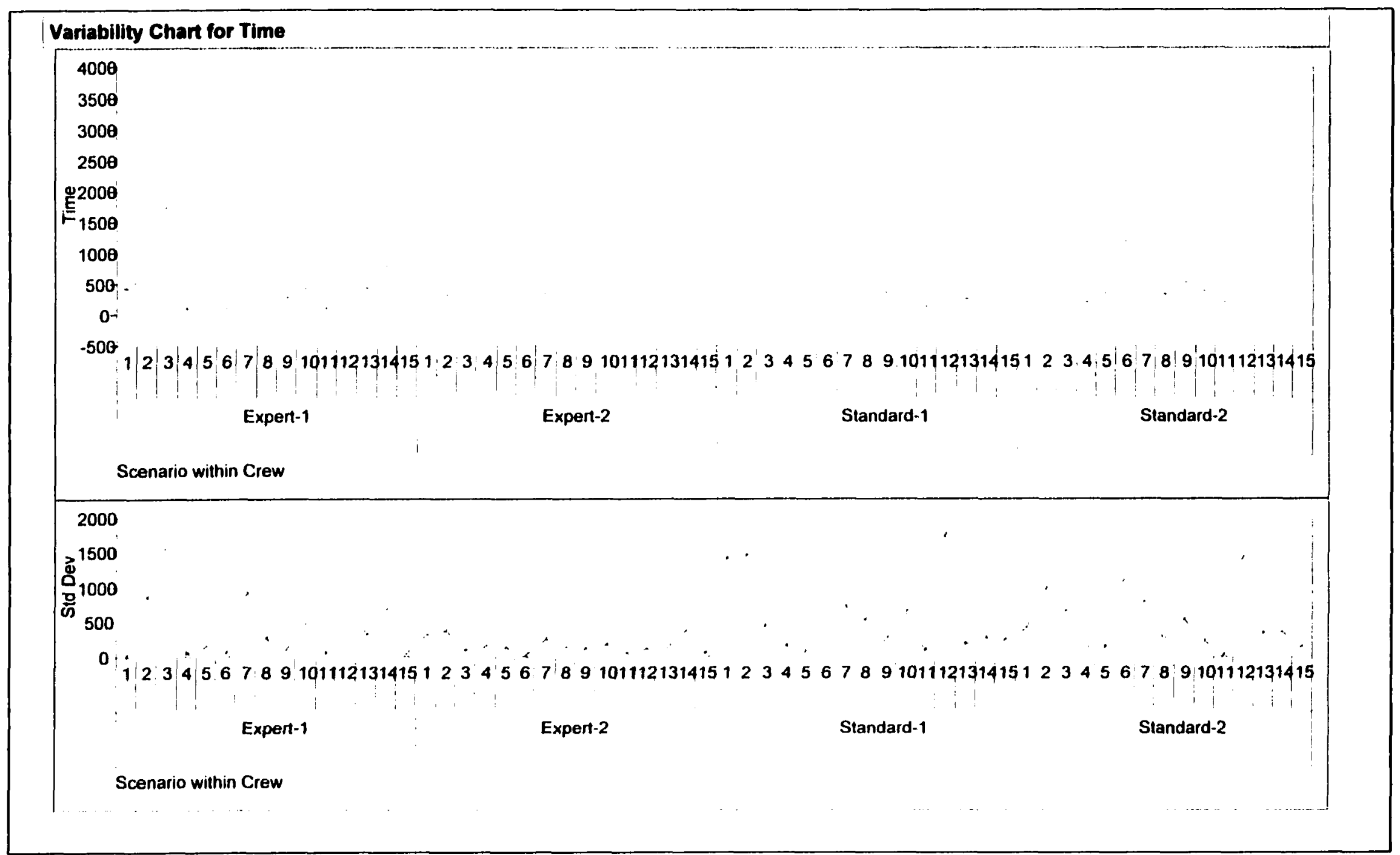

Figure 6.5: Variability charts for scenario vs. time grouped by crew. Top chart shows resolution means for each crew. 


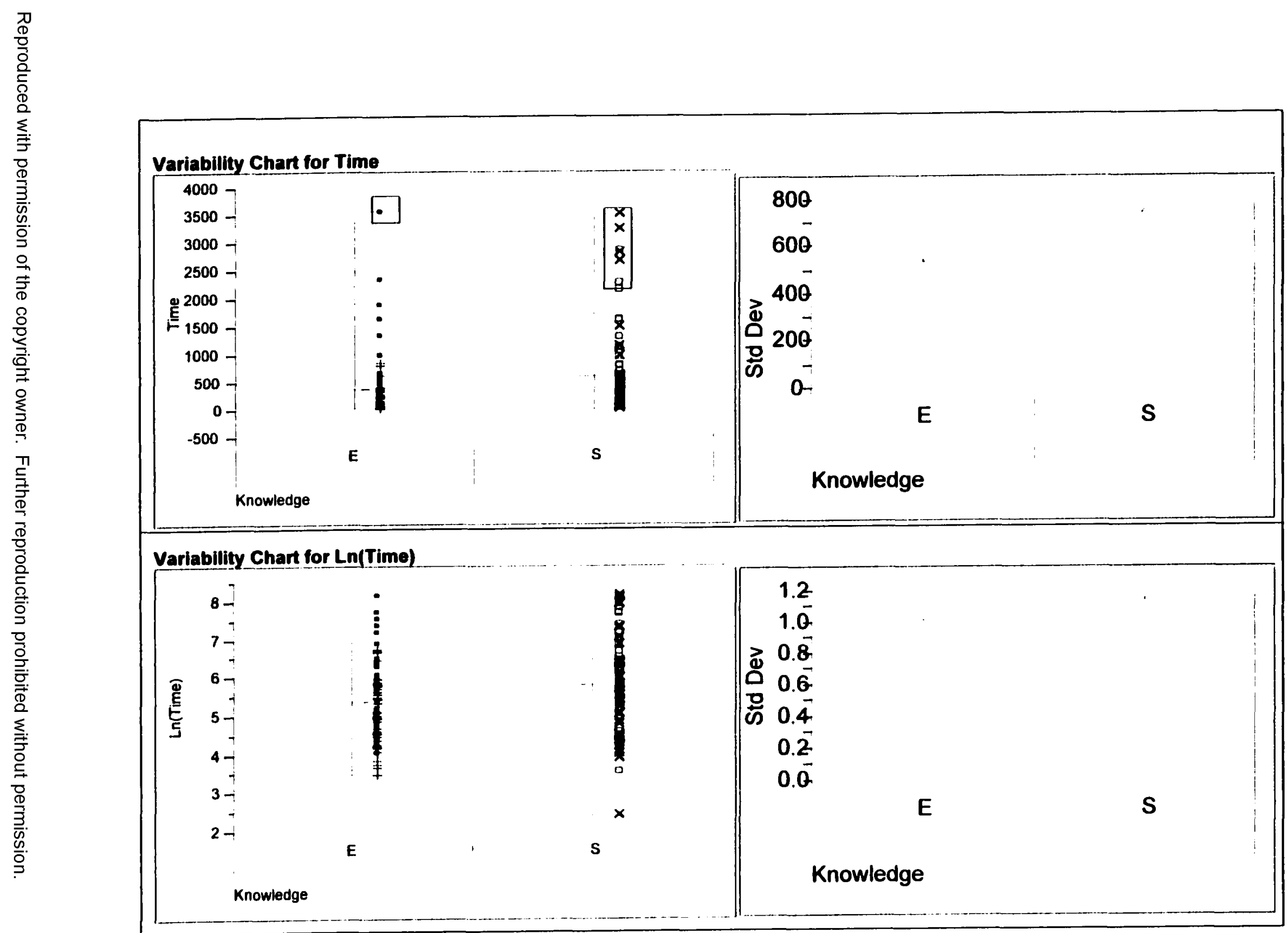

Figure 6.6: Variability charts for knowledge vs. time and In(time). Symbols represent individual crew observations. 


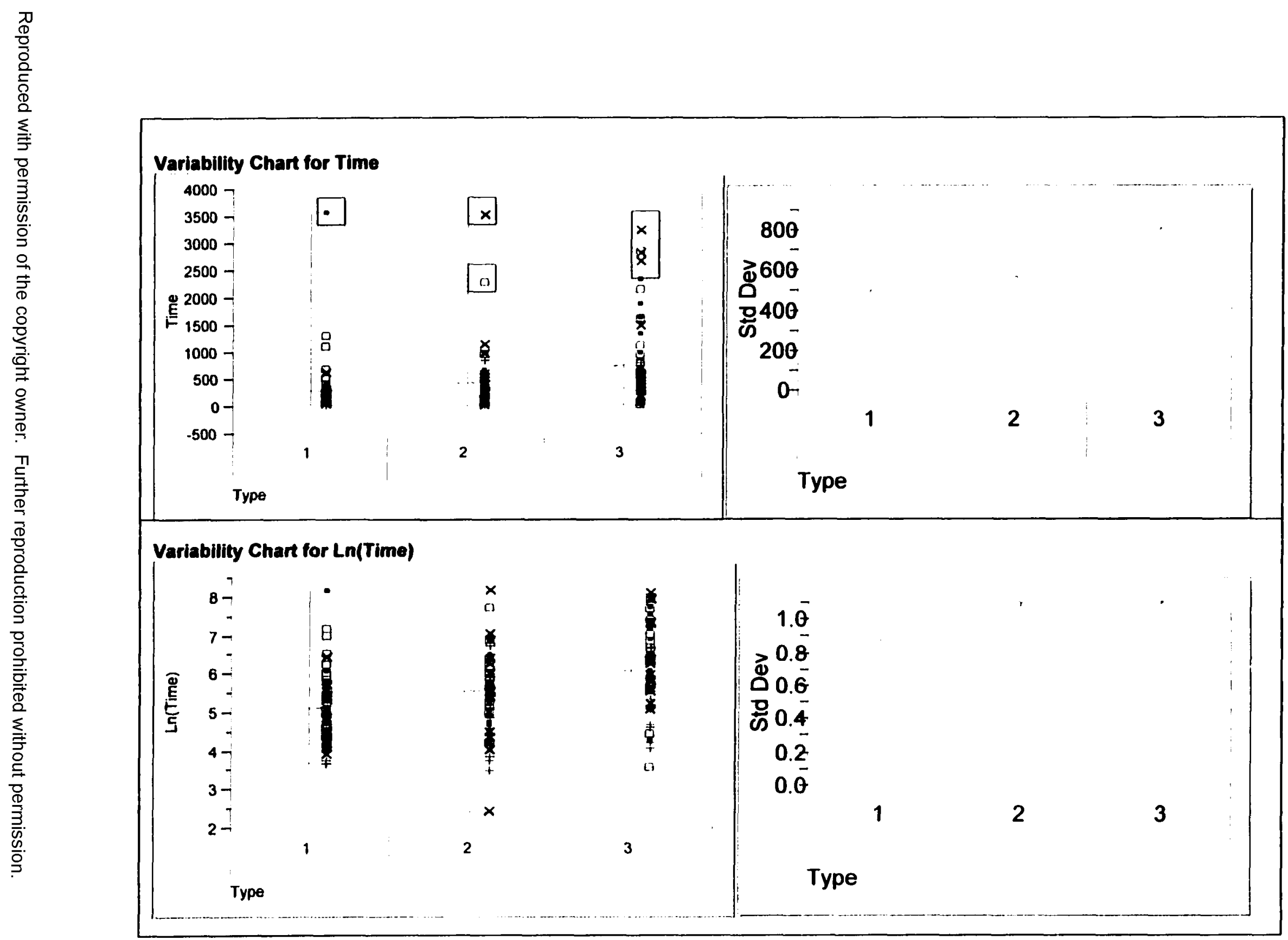

Figure 6.7: Variability charts for type vs. time and In(time). Symbols represent individual crew observations. 
The colored markers in the variability charts represent the individual observations of the crews for each anomaly resolution time. Note that in Figures 6.4 and 6.5 , the scenario to scenario variation appears large compared to the within-scenario variation, validating the use of scenario as a blocking variable. Figure 6.6 shows definite variation in the expert crew knowledge levels, demonstrating that one expert (expert 2) is much more consistent in resolution ability than the other expert and both the standard crews. This expert crew variation was also evident in the data analysis and will be discussed in section 6.2.3.3. Examining the type variability (Figure 6.7), it is also apparent that resolution time increases with the difficulty level. There is notable variation between Types 1 and 2, while there is significantly less variation between Types 2 and 3.

Initially, an Analysis of Variance (ANOVA) was performed using design factors of knowledge (expert/standard), type (1, 2, or 3), and crew with nested under knowledge. A model for In(time) was developed that included these three factors, the interaction of type and knowledge, and scenario as a blocking variable. The purpose was to examine the overall effects of the factors and assess the contribution of the random effect of crew. This was of concern since the Proportional Hazards Model platform in JMP Professional does not permit for inclusion of random effects. As seen in Figure 6.8, it was determined that this random effect did have a small contribution $(0.080)$ to the residual variation of the overall variance component $(0.956)$, accounting for roughly $10 \%$ of the total variation. However, as mentioned earlier, JMP does not permit analysis of random effects in the Proportional Hazards analysis. Even though the random component of the variation could not be estimated in the final proportional hazards model, the nested effect of crew within knowledge was included since there was a definite contribution of the nesting effect of crew within knowledge. The implication of this decision is that results relating to crew differences within knowledge groups from the 
proportional hazards analysis will apply specifically to the four crews involved in this experiment.

$\begin{array}{lcr}\text { Variance Component Estimates } & \\ \text { Component } & \text { Var Comp Est } & \text { Percent of Total } \\ \text { Crew(Knowledge]\&Random } & 0.080347 & 8.405 \\ \text { Residual } & 0.875539 & 91.595 \\ \text { Total } & 0.955886 & 100.000 \\ \text { These estimates based on equating Mean Squares to Expected Value. } & \end{array}$

Figure 6.8: Random effects of crew nested within knowledge

6.22 Cox's Proportional Hazards Analysis. Final time data analysis was accomplished using Cox's Proportional Hazards model, where "time to event" in this study is resolution time (rather than "survival time"). The time data during this analysis was not transformed, since the Proportional Hazards analysis models the raw data itself. This is useful, as it is much more meaningful to interpret the data in pure time versus In(time). As discussed in Chapter 5, the model itself is partially non-parametric and partially parametric. The nonparametric portion is the baseline hazard, and this function can virtually be of any form. The parametric part is the linear component; namely, the linear model for the covariates. Because of these model characteristics, the nature of the underlying distribution is independent of the proportional hazards model fit.

The fact that the survival curves for knowledge and type do not cross (Figures 6.9 and 6.10) is a major indicator that the $\mathrm{CPH}$ model is appropriate, namely, this suggests that the hazard functions may be proportional (Selvin, 1995). The Log-Rank and Wilcoxon tests for the log-normal transformed data in JMP tests the hypothesis that the survival functions are the same across the knowledge and type groups. The high significance of these two tests $\left(p_{\text {knoweldge }}=0.0058\right.$ and 0.0057 , respectively: $p_{\text {type }}<$ 0.0001 for both) indicates the null hypothesis that the groups are the same should be 
rejected. The proportionality and parallel nature of knowledge group survival curves can be verified pictorially, as seen in Figures 6.9 and 6.10 .

To verify that Cox's Proportional Hazards model is suitable for the analysis, it is possible to construct various plots of the expert and standard crews for comparison. Namely, if the Proportional Hazards model is fit for both groups and compared to the Kaplan-Meier Survival curves for the same crews, we can verify that the Cox's Proportional hazards model is a good fit and is an appropriate selection. Additionally, $\log -\log$ transformations of the Proportional Hazards model fit can be compared for each group, expert and standard (Selvin, 1991). This should show, in all cases, that the functions are approximately additive. 


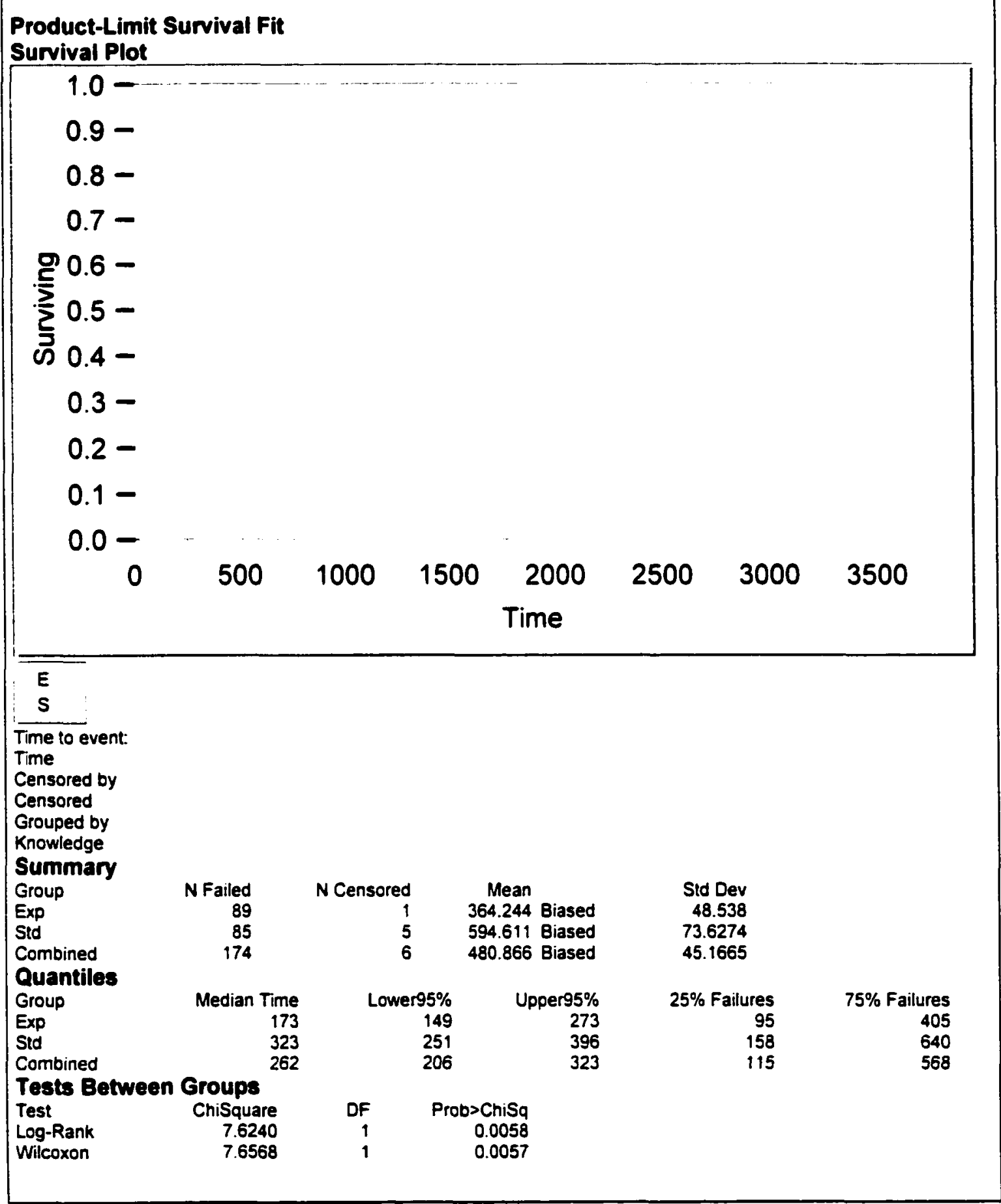

Figure 6.9: Survival plot of time data grouped by knowledge level 


\section{Product-Limit Survival Fit}

Survival Plot

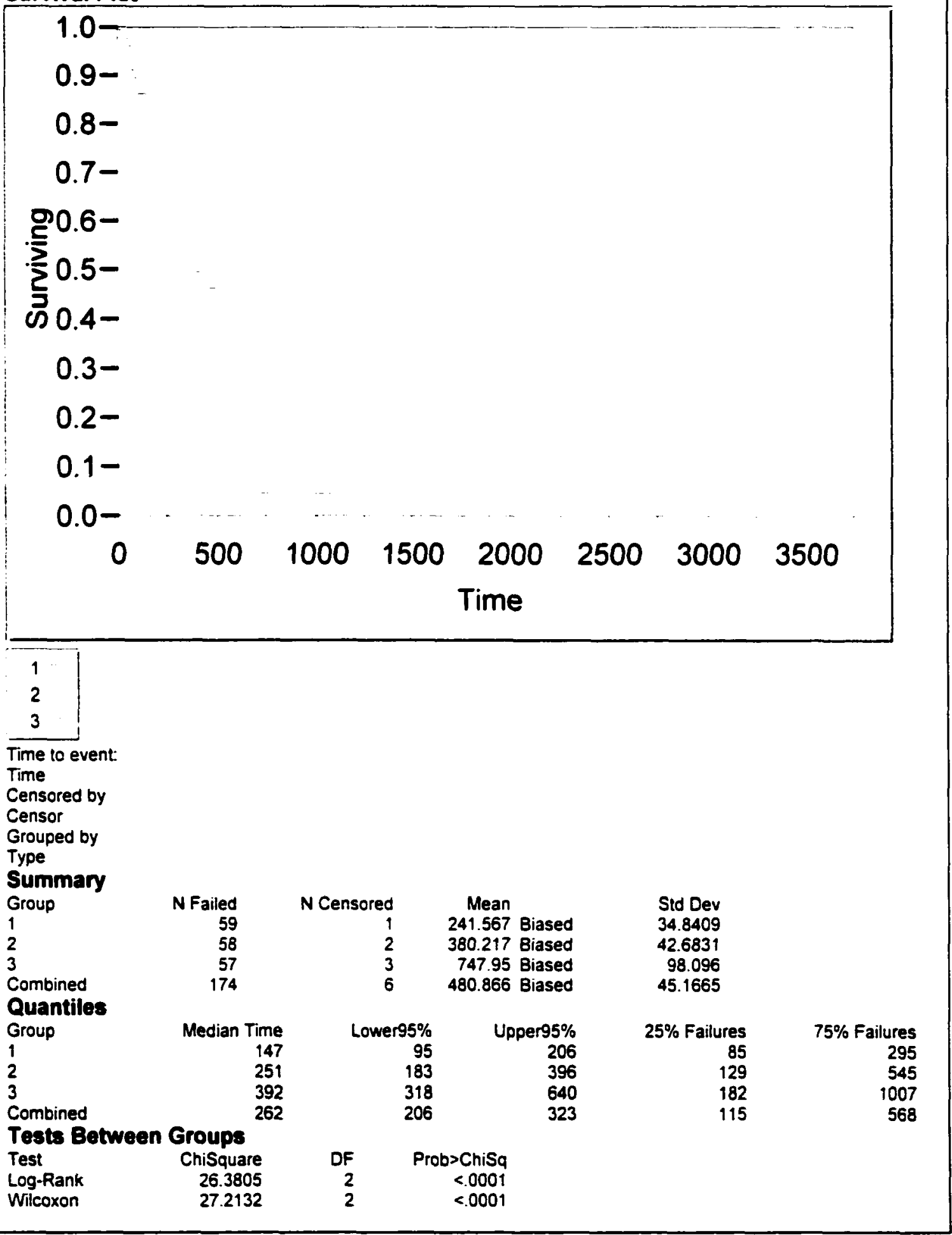

Figure 6.10: Survival plot of time data grouped by anomaly type 
Kaplan-Meier and Cox's Proportional Hazards Survival Functions Versus Resolution Time, Combined Crews

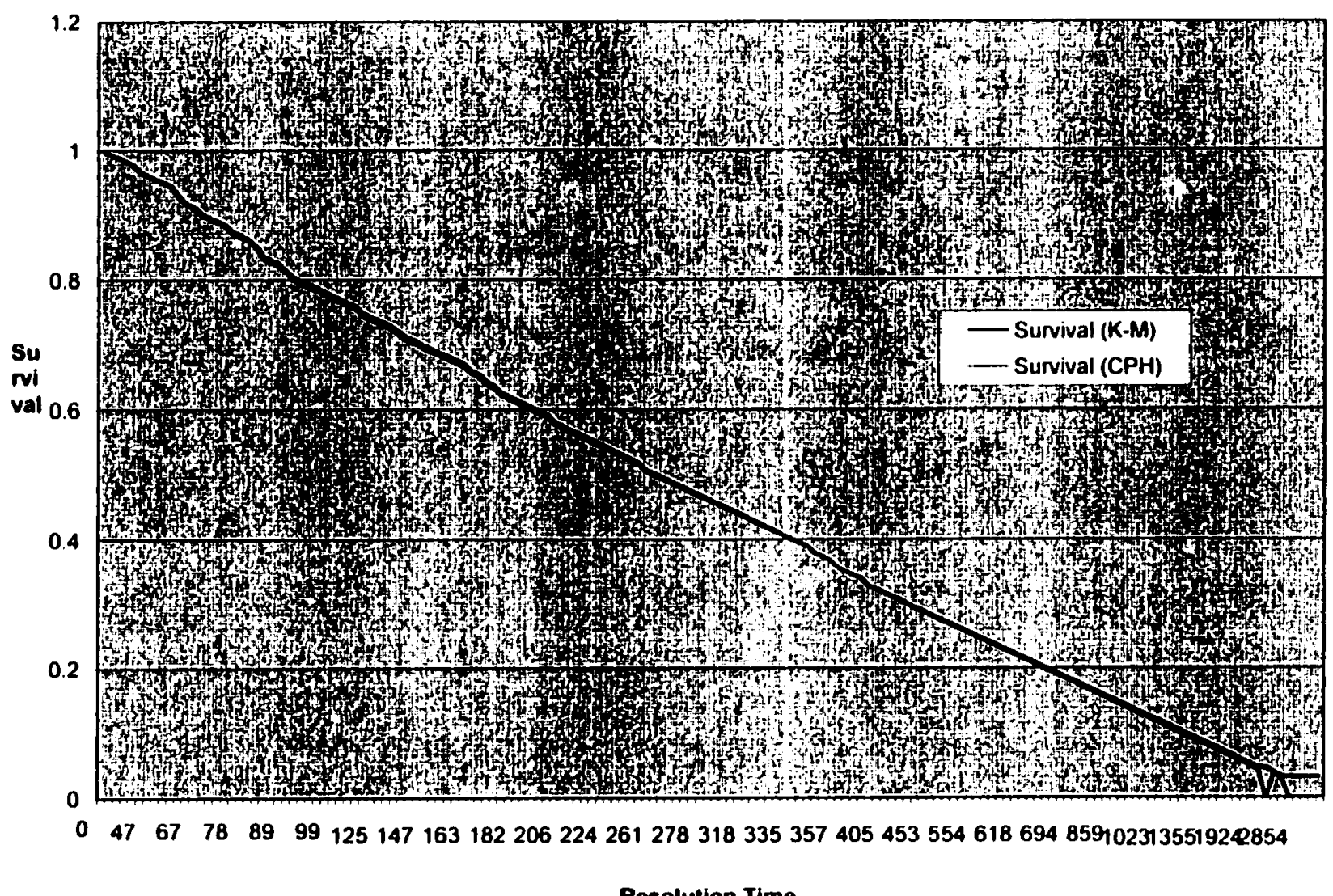

Resolution Time

Figure 6.11 
Kaplan-Meier and Cox's Proportional Hazards Survival Functions Versus Resolution Time, Standard Crews

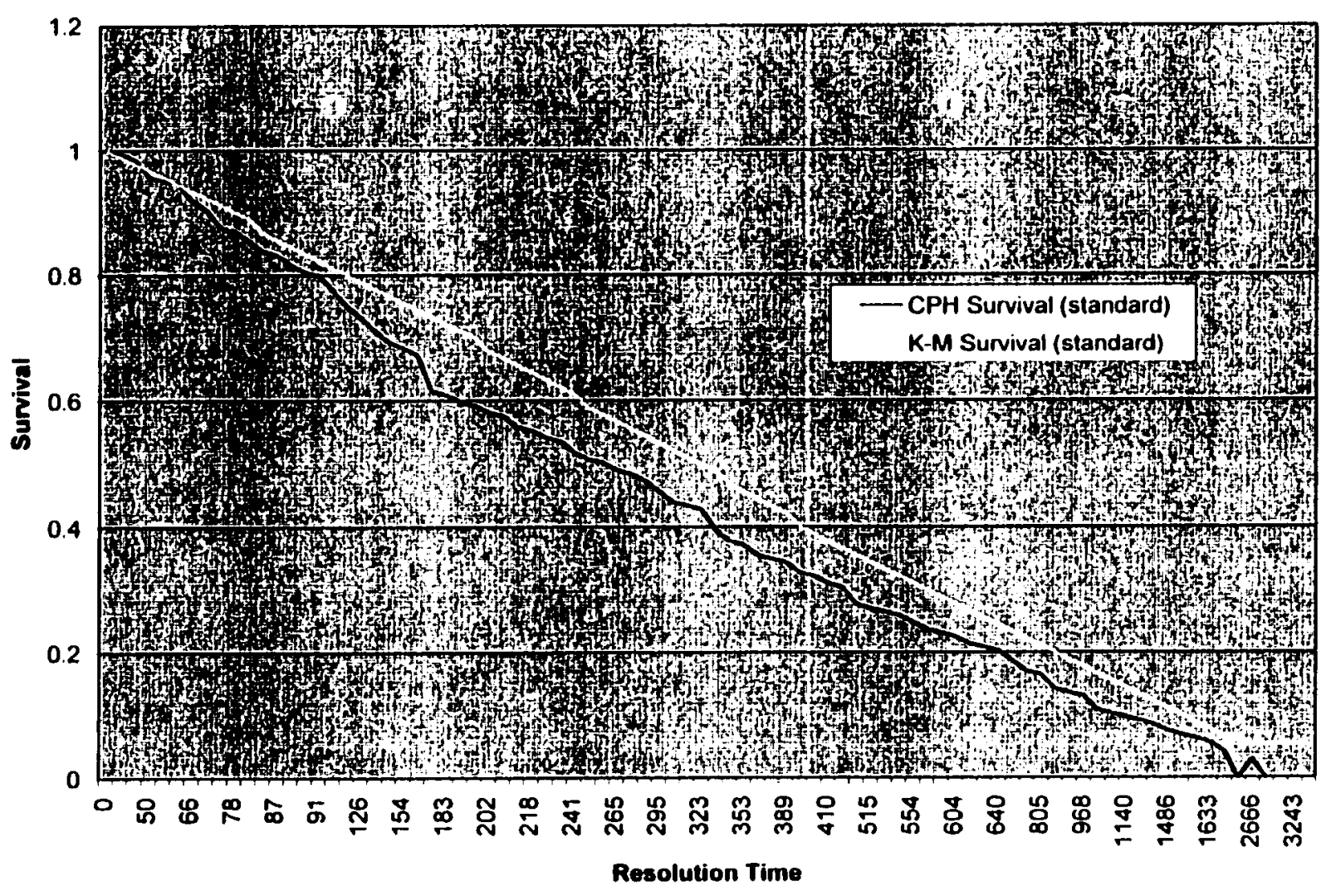

Figure 6.12 
Kaplan-Meier and Cox's Proportional Hazards Survival Functions Versus Resolution Time, Expert Crows

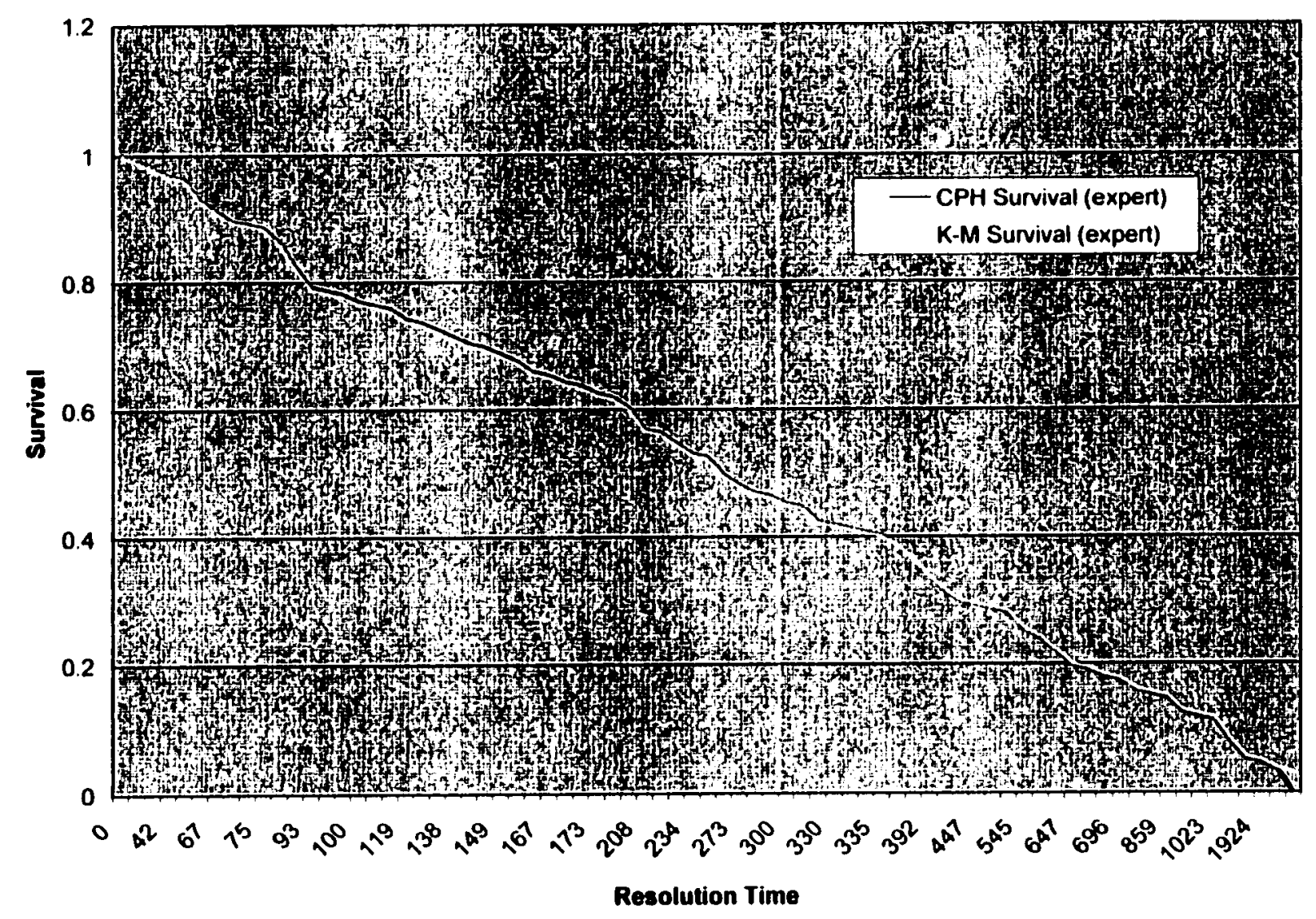

Figure 6.13 


\section{Log-Log Transformation of Kaplan-Meier and Cox's Proportional Hazards Survival Functions}

Versus Resolution Time, Combined Crews

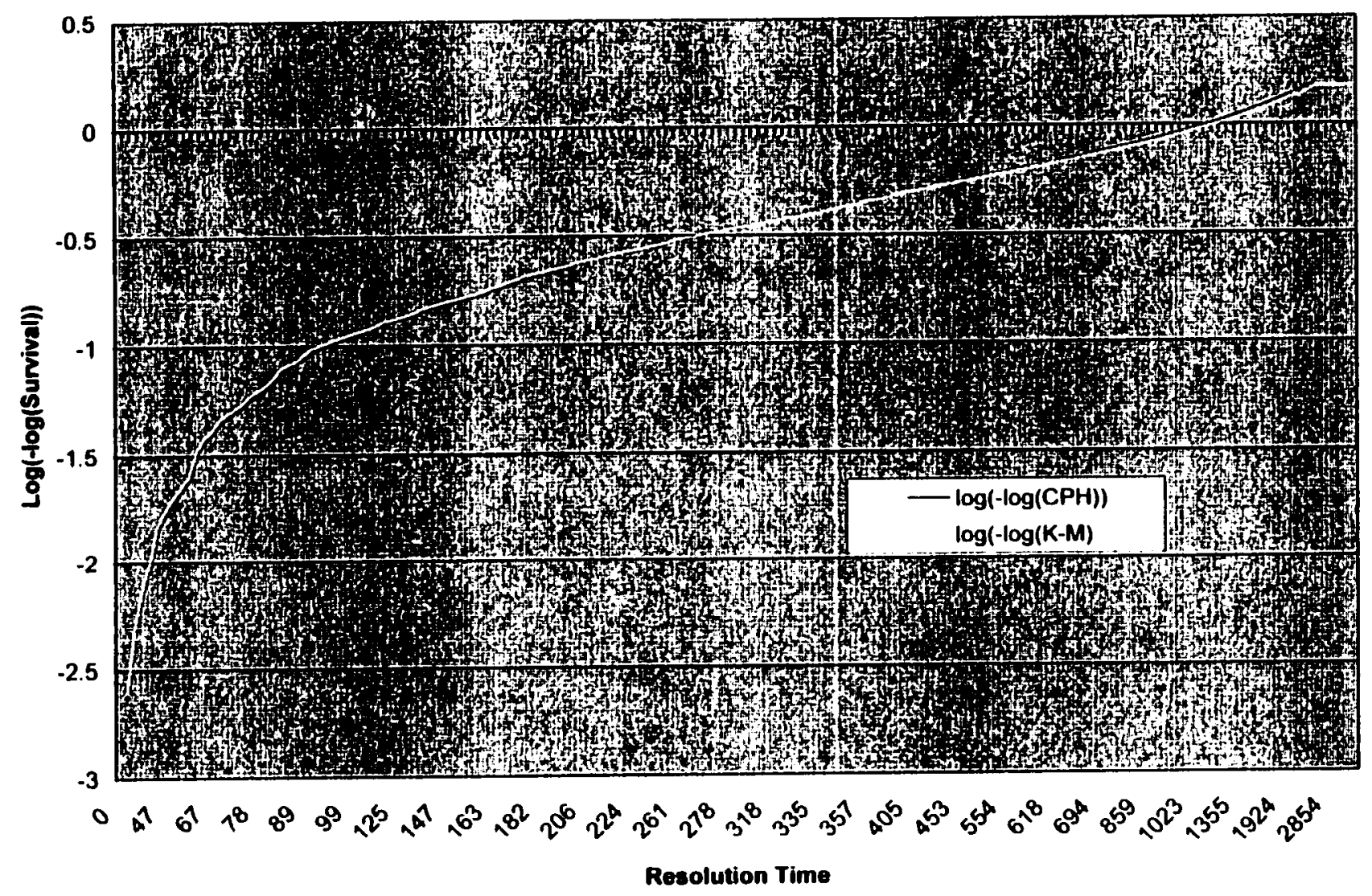

Figure 6.14 


\section{Log-Log Transformation of Kaplan-Meier and Cox's Proportional Hazards Survival Functions Versus Resolution Time, Standard Crews}

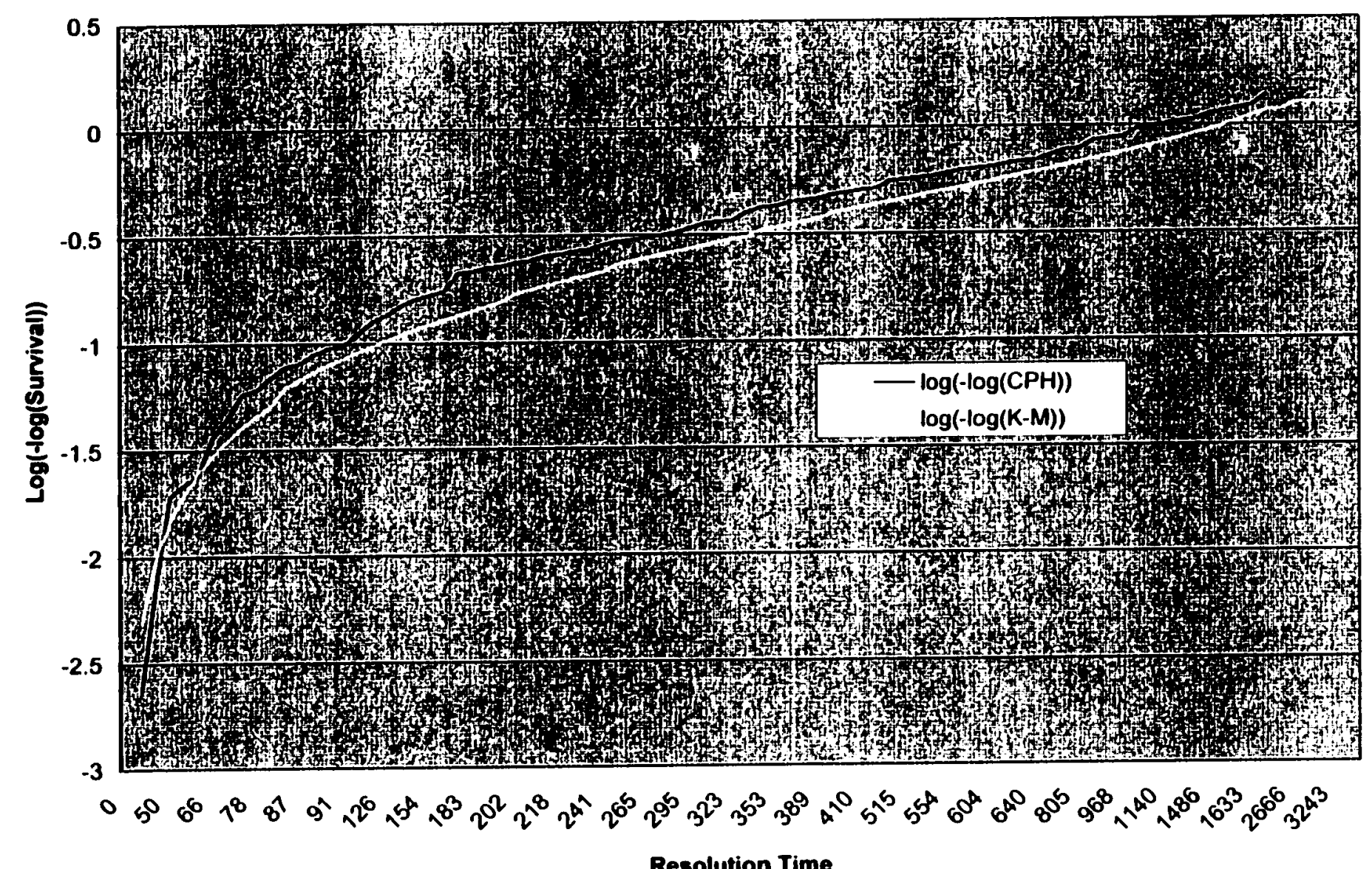

Resolution Time

Figure 6.15 
Log-Log Transformation of Kaplan-Meier and Cox's Proportional Hazards Survival Functions Versus Resolution Time, Expert Crews

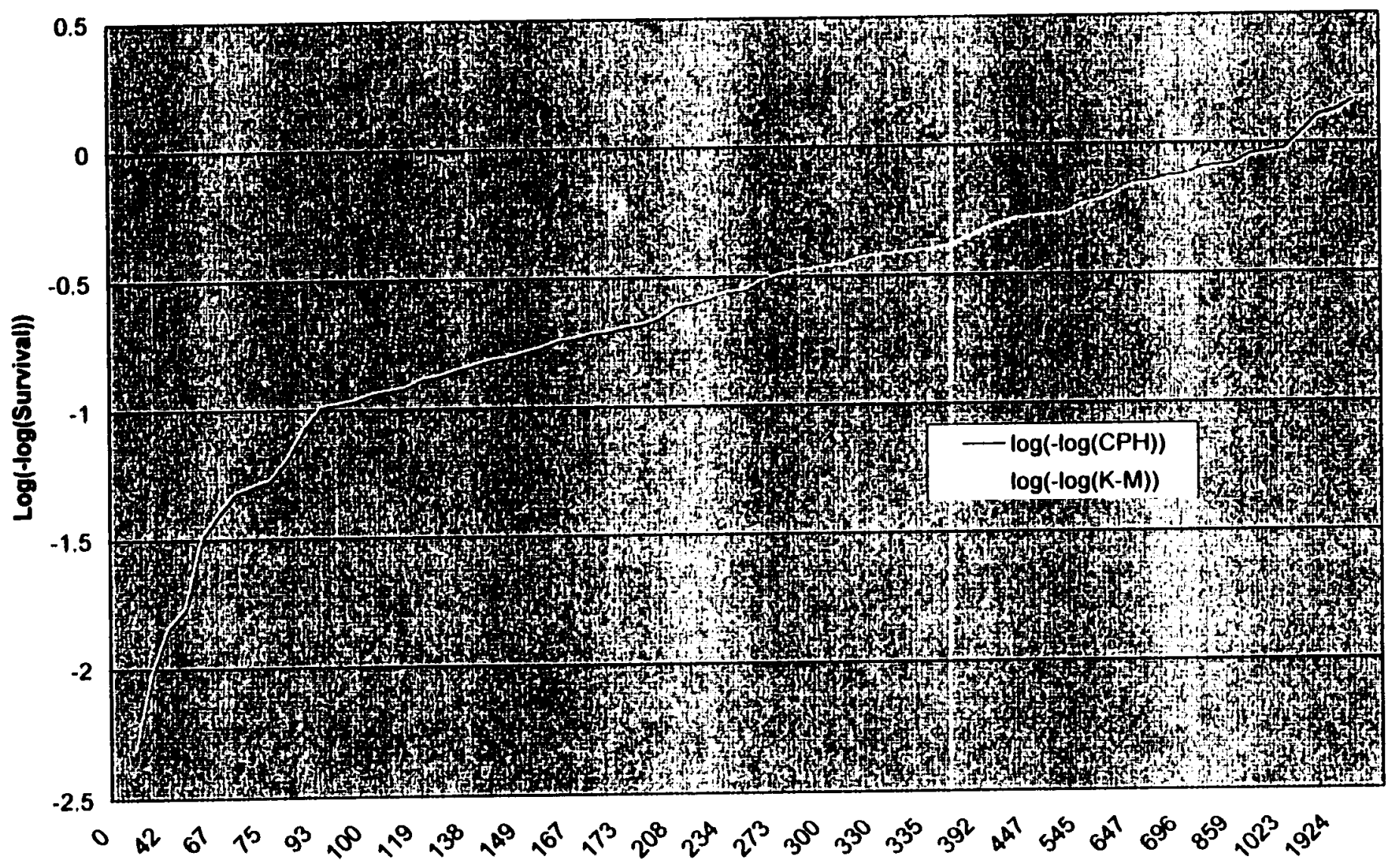

Resolution Time

Figure 6.16 
6.2.2.1 Full model. The initial iteration of the Cox's Proportional Hazards model in JMP contained design factors of knowledge, scenario, crew (nested within knowledge) type and knowledge "type, with time as the response variable. Six of the 180 observations were censored, meaning that the anomaly was not resolved within the scenario length. Censored observations occurred in scenarios 1, 3,6 and 12. This censoring effect was denoted in the survival analysis model as "censored".

In this first analysis, it was immediately obvious that while scenario appeared significant ( $p=0.0003$ ), it used 14 degrees out of 21 total degrees of freedom that could instead be used to estimate error. There was a definite trade-off between blocking by scenario and leaving the effect in the model and freeing up the related degrees of freedom by excluding it. Scenario could be arguably removed since when modeling for real-life events, "scenarios" are not fixed and anomaly arrangements occur randomly. Also, the model excluding scenario provides greater ease of interpretation. Subsequent trial models were executed leaving the scenario factor either intact or excluded.

In the full-model analysis, the only non-significant effect was the interaction of type and knowledge; all other p-values were significant in terms of the test alpha value of 0.05 .

Therefore, a final model was fit excluding scenario and the interaction term (Figure 6.17). This model appeared to adequately describe the data in the absence of the scenario factor and the non-significant type"knowledge interaction term, and was consequently selected as the final version. The survival plots for both the full and final models were characteristic of typical survival data. Details on interpreting parameter estimates, confidence intervals, and risk ratios will be provided later in the chapter.

6.2.2.2 Final model. The final model (Figure 6.17) has a total of 180 observations (174 


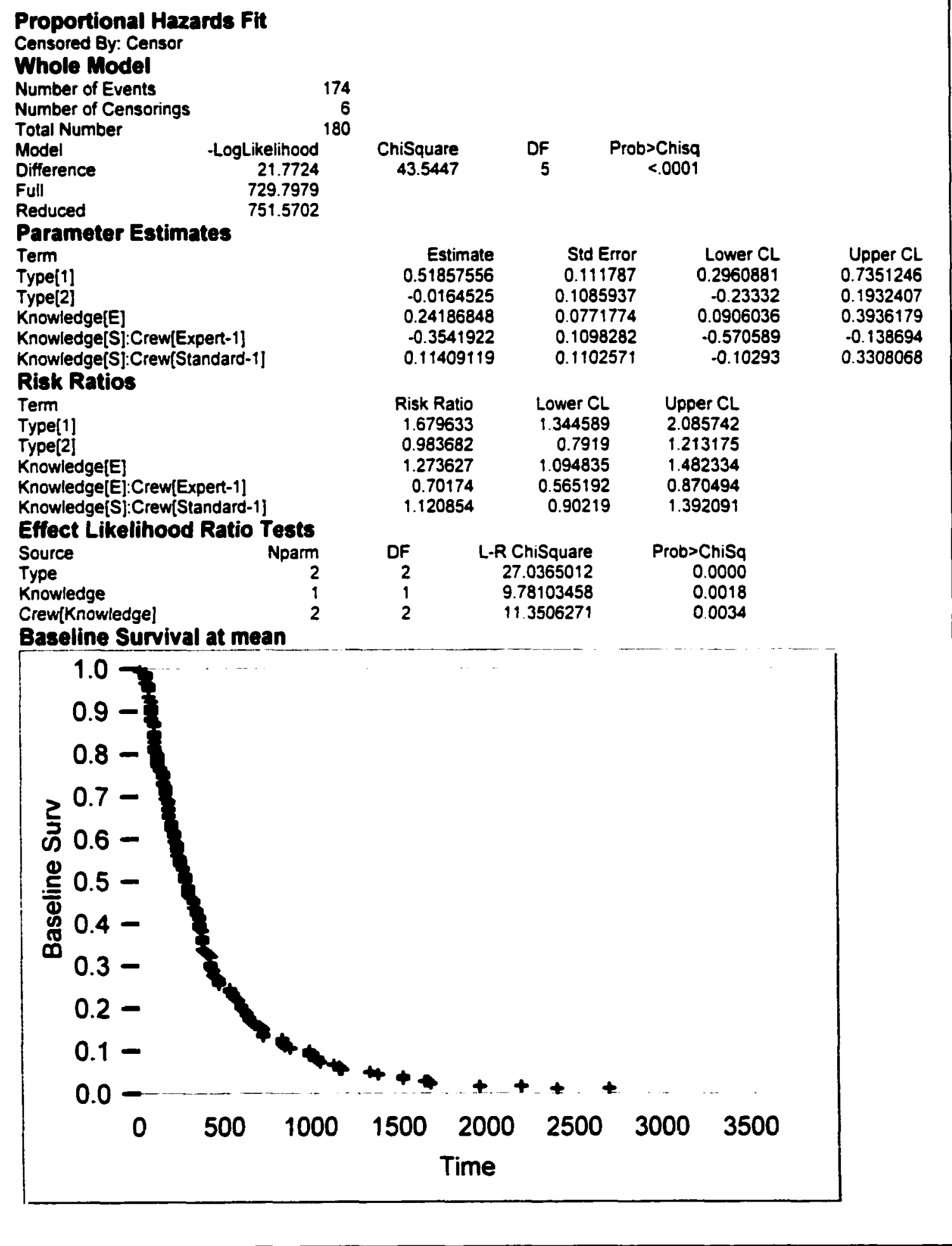

Figure 6.17: Cox's Proportional Hazards Model; baseline survival plot (final model) 
complete/6 censored) and five degrees of freedom (DF). One DF results from the two knowledge levels (expert/standard), two DF from anomaly type leveis (1,2, and 3), and two from the crew effect (two expert and two standard crews nested within knowledge).

The final proportional hazards model can be mathematically expressed as:

$$
\lambda_{j}(t)=\lambda_{0}(t) \exp \left\{b_{1} x_{1 j}+b_{2} x_{2 j}+b_{3} x_{3 j}+b_{4} x_{4 j}+b_{5} x_{5 j}\right\}
$$

or specifically,

$$
\begin{aligned}
& \lambda_{j}(t)=\lambda_{0}(t) \exp \left\{b_{1} x_{\text {knowledge }}+b_{2} x_{\text {type(1) }}+b_{3} x_{\text {type(2) }}+\right. \\
& \left.b_{4} x_{\text {[crew(exp)[knowledge }]}+b_{5} x_{\text {[crew(std)[knowledge }]}\right\}
\end{aligned}
$$

where $\lambda_{j}(t)$ represents the $j^{\text {th }}$ hazard function and $\lambda_{0}(t)$ is the baseline hazard function.

The parameter estimates and indicator variables can be described as:

- Knowledge: parameter estimate $=b_{1}$, indicator variable $x_{1 j}$, where

$$
x_{1 j}=\left\{\begin{array}{l}
1 \text { if high } \\
0, \text { otherwise }
\end{array}\right.
$$

- Type: parameter estimates $=b_{2}$, (type 1), $b_{3}$ (type 2)

indicator variables $x_{2 i}$ (type 1 ), $x_{3 j}$ (type 2 ), where

$$
\begin{aligned}
& x_{2 j}=\left\{\begin{array}{l}
1 \text { if type }=1 \\
0, \text { otherwise }
\end{array}\right. \\
& x_{3 j}=\left\{\begin{array}{l}
1 \text { if type }=2 \\
0, \text { otherwise }
\end{array}\right.
\end{aligned}
$$

Type 3 denoted when $x_{2 j}=x_{3 j}=0$ 


\section{- Crew, Nested Within Knowledge: parameter estimates = $b_{4}$ (expert crew 1 (knowledge expert)), $b_{5}$ (standard crew 1 (knowledge expert))}

One method of interpreting the JMP data is analysis of the Effect Likelihood Ratio Tests, which indicate whether an effect is statistically significant or not. This test compares the two models using the likelihood function, and fits them based on maximum likelihood. One model assumes that the response is constrained, while the other model is not constrained. This means that the model is based on either including or not including a factor in modeling the response. Therefore, a response containing no factors $\left(H_{0}\right)$ is compared to a response including a factor, such as knowledge $\left(H_{a}\right)$ to determine if significant differences exist across the constrained groups. Twice the difference of the log-likelihoods is a chi-square statistic that is used for testing the null hypothesis. This chi-square statistic can be interpreted similarly to any test statistic according to p-value significance of at a pre-specified alpha level.

In survival analysis, a meaningful interpretation of the contributions of main effects is provided by the risk ratios (RR). Risk ratios (also called hazard ratios) are the relative rates of hazard between groups. Risk ratios can also be interpreted as an estimate of the risk of failure at a given time (Selvin, 1995). However, in this case study, the risk of failure is translated into the opportunity for anomaly resolution. When two hazard functions are identical, the hazard ratio is $c=1$, or $c=\lambda_{1} / \lambda_{0}=1$. Hazard ratios in the region of $c=1$, or hazard ratio confidence intervals (Cl's) containing the value of one result in an inability to reject the null hypothesis $H_{0}$ (no difference exists among groups) in favor of the alternative, $\mathrm{H}_{\mathrm{a}}$ (a difference exists).

It is important to note that choice of data interpretation by either Effect Likelihood Ratios or Risk Ratios results in no loss of data or explanation, since they vary only by an exponential transformation. It is solely a description technique preference. 


\subsubsection{Proportional Hazards Results.}

6.2.3.1 Type. The main effect of anomaly type shows a highly significant Effect Likelihood Ratio Test of $p \approx 0$, indicating that the null hypothesis of anomaly resolution times being identical across different anomaly types should be rejected. Type 3 anomalies were compared against types 1 and 2 . When examining the risk ratios for the main effect of type, all confidence intervals contain the value of one (cannot reject $H_{0}$ ) with exception of Type[1]. This provides evidence of a significant difference across hazard functions, indicating Type 1 anomalies were resolved faster than Types 2 and 3.

More specifically, operators resolved Type 1 anomalies 1.680 times faster on average than Type 3 anomalies. Type 2 anomalies were resolved in roughly the same amount of time as Type 3 's $(R R=0.984 \cong 1, R R C l[0.792,1.213])$, indicating that the difficulty level between the two types is similar among all operators, or that the initial differentiation between anomaly difficulties of types 2 and 3 was inaccurate. In this instance, subjective data collected during the experiment will prove to be extremely useful, as anomaly difficulty should not be represented solely by resolution time.

6.2.3.2 Knowledge. The most important main effect tested was knowledge. Knowledge main effects were significant at a p-value of 0.0016 . This verifies there was a significant difference in resolution ability between knowledge groups, and the hazard functions were not identical $(R R=1.274, R R C l[1.095,1.482])$. This would lead to the rejection of the null hypothesis that there is no difference in expert or standard groups' time to resolve.

Experts were 1.274 times faster overall ( $R R C l[1.095,1.482]$ ) in resolving anomalies than the standard group. Experts were also 2.140 times faster in resolving Type 1 anomalies than Type 3's $\left(C_{\text {exp }}{ }^{*} C_{\text {type }}{ }_{1}=e^{0.242} e^{0.519}=(1.680)(1.274)=2.14\right)$, 
whereas standard crews were 1.680 times faster in resolving Type 1's than Type 3's $\left(C_{\text {stc }}{ }^{*} C_{\text {type } 1}=e^{0} e^{0.519}=(1)(1.680)=1.680\right)$. However, for type 2 anomaly resolution, again there was not a noted difference between that of resolving type 2's and 3's.

To make this interpretation more meaningful, suppose all crews had 100 anomalies to resolve in a certain amount of time. If the experts resolved all 100 anomalies, the standard crews would solve only about 78 of those anomalies in that same time, regardless of anomaly type. If there were multiple type 1 anomalies for the crews to address, the experts would be able to correctly resolve 215 anomalies in the same time the standard crews would be able to complete 168 anomalies. Subsequently, experts would correct 126 type 2's and 128 type 3's in the same amount of time that the standard crews would require to resolve 98 and 100 type 2's and 3's, respectively. The Risk Ratio calculations are shown in Figure 6.18; all ratios are calculated relative to Type 3 and their interactions with Type 3.

6.2.3.3 Crew effects. The analysis verifies that there is no significant difference in the standard crews nested within the knowledge levels, indicating that the two specific crews chosen within the standard knowledge level do not appear to differ. The reader should be reminded that crew, in this analysis, is being treated as a fixed effect rather than a random effect. However, there is a significant difference between the expert crews. This indicates non-homogeneity of ability between the experts, which is actually not surprising: expert 1 has 37 months on crew, whereas expert 2 has 159 months of experience. The small population of expert operators made it impossible to guarantee homogeneity of experience levels. Therefore, although there is a disparity between experience levels, the data is still meaningful and interpretable. 


\begin{tabular}{|l|c|c|c|}
\hline \multicolumn{1}{|c|}{ Paramoter } & Paramoter Estimate & Calculations & Risk Rotio \\
\hline Type 1 & 0.519 & $\mathrm{e}^{0.519}$ & 1.680 \\
\hline Type 2 & -0.016 & $\mathrm{e}^{-0.016}$ & 0.984 \\
\hline Type 3 & 0 & $\mathrm{e}^{0}$ & 1.000 \\
\hline Knowledge (expert) & 0.242 & $\mathrm{e}^{0.242}$ & 1.274 \\
\hline Knowledge (standard) & 0 & $\mathrm{e}^{0}$ & 1.000 \\
\hline Knowledge (exp)* Type (1) & & $\mathrm{e}^{0.242} \mathrm{e}^{0.519}$ & 2.140 \\
\hline Knowledge (std) * Type (1) & & $\mathrm{e}^{0} \mathrm{e}^{0.519}$ & 1.680 \\
\hline Knowledge (exp)* Type (2) & & $\mathrm{e}^{0.242} \mathrm{e}^{0.016}$ & 1.254 \\
\hline Knowledge (std) * Type (2) & & $\mathrm{e}^{0} \mathrm{e}^{-0.016}$ & 0.984 \\
\hline Knowledge (exp)* Type (3) & & $\mathrm{e}^{0.242} \mathrm{e}^{0}$ & 1.274 \\
\hline
\end{tabular}

Fiqure 6.18: Risk Ratio Calculations

\subsection{Subjective Data}

6.3.1 SWAT Analysis. Subjective data collected following each of the 15 scenarios for all four test groups was analyzed using the Subjective Workload Assessment Technique (SWAT) software package, version 3.1. This software required an older PC with a DOS version of 6.0 or earlier. In addition, the ANSI.SYS file must be added to the CONFIG.SYS prior to booting into DOS mode to ensure proper program operation.

\subsubsection{SWAT Data Interpretation.}

6.3.2.1 Rescaling. The participants performed the card sort of 27 cards in order to arrive at a scale describing perceived difficulty of certain tasks. These cards generically described the complexity of arbitrary tasks, and the subjects were requested to rank order these cards. Once this was completed, the card order was entered in the SWAT software for each member, after which the rescaled data was computed for all 8 participants. The solution translates the Time Effort (T), Mental Effort (E), and Psychological Stress (S) ratings into single, numerical values. For example, if a subject 
ranks an event with a T-E-S difficulty of 3-3-3, the equivalent rescaled value is 100 . This solution can be viewed in Figure 6.19.

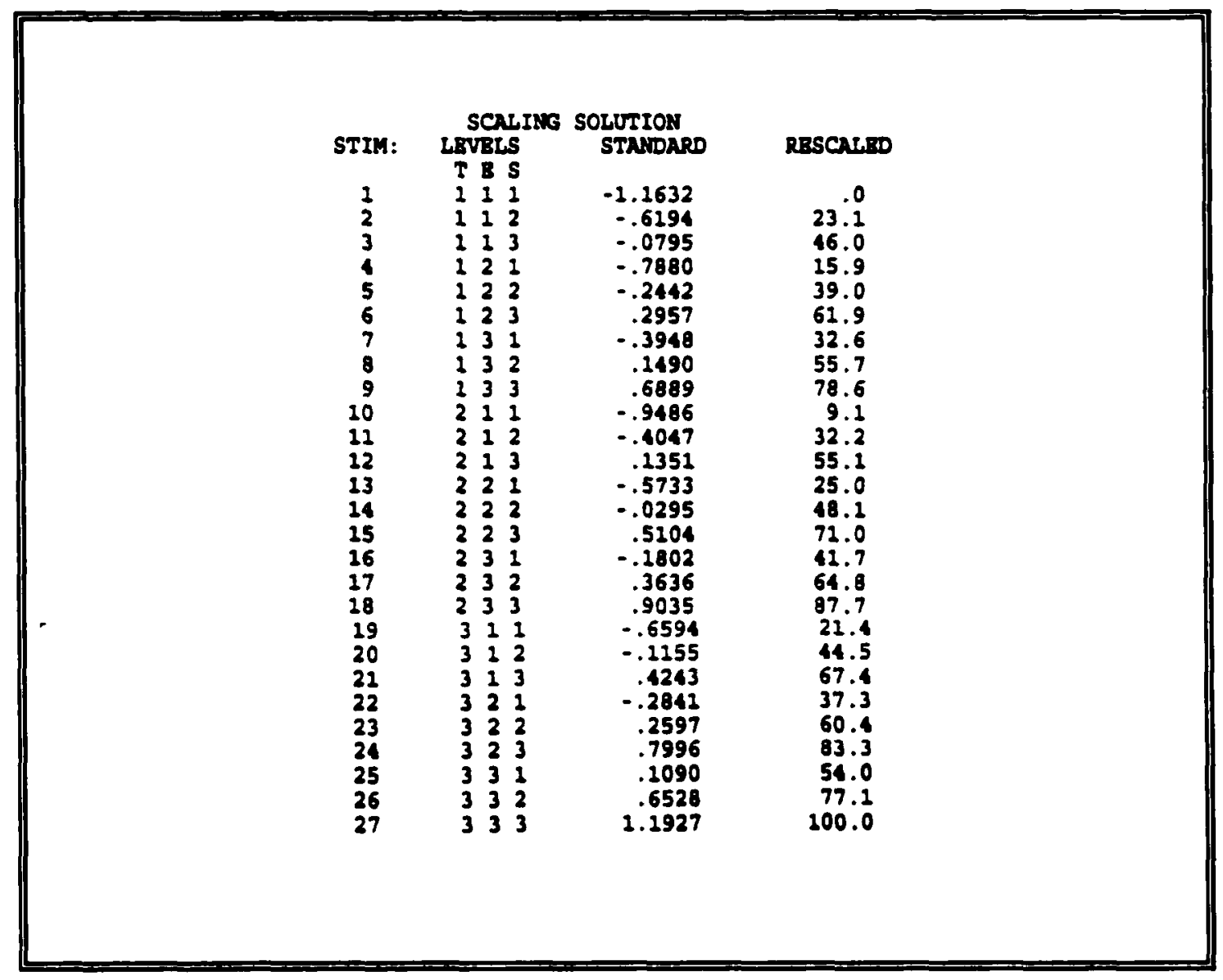

\section{Figure 6.19: SWAT Rescaling}

The experimenter has the option of applying a group solution (all group members have the same rating scale), a prototype solution (the experimenter can create subject subgroup solutions), or individual solutions. This decision is based on the value of the Kendall's Coefficient of Concordance. The coefficient describes how well the subjects are in agreement with each other in terms of perceived difficulty. If there is considerable agreement among the participants, the coefficient should be greater than 0.75 . It is then 
up to the experimenter as to which option best describes the data and satisfies the experiment goals. The coefficient is determined during the prototype correlations analysis, where a Spearman's rank order correlation is performed on each of the subject's rank ordered data. The rankings are associated with the possible prototype groups, and the correlations are indicative of the relative importance each subject assigns to the three dimensions of Time, Effort and Stress.

The Kendall's Coefficient of Concordance for the study data was $\mathbf{0 . 8 8 3}$, indicating a high correlation between how the subjects defined "difficulty" (Figure 6.20). Also note that the suggested prototype for the majority of subjects was $S$, meaning that six out of eight participants thought that Psychological Stress was the most appropriate descriptor of effort. Two of the eight subjects (subjects 2 and 6) thought that Mental Effort was the most significant dimension; however, the overall correlation among the group was high enough so that they also matched the Stress prototype. This enabled use of a group solution since, statistically, it appeared that all subjects were in general agreement about the ordering of the cards and the relative importance of the three dimensions.

A plot was also generated in the SWAT analysis (Figure 6.21) to indicate the goodness of fit of the rescaled values. The appropriate plot is linearly decreasing from left to right, and data points not laying on the line indicate cards that were displaced from the pattern. The entire SWAT analysis can be seen in Appendix A, Raw Data. 
Figure 6.20: Prototype Analysis and Subject Correlation

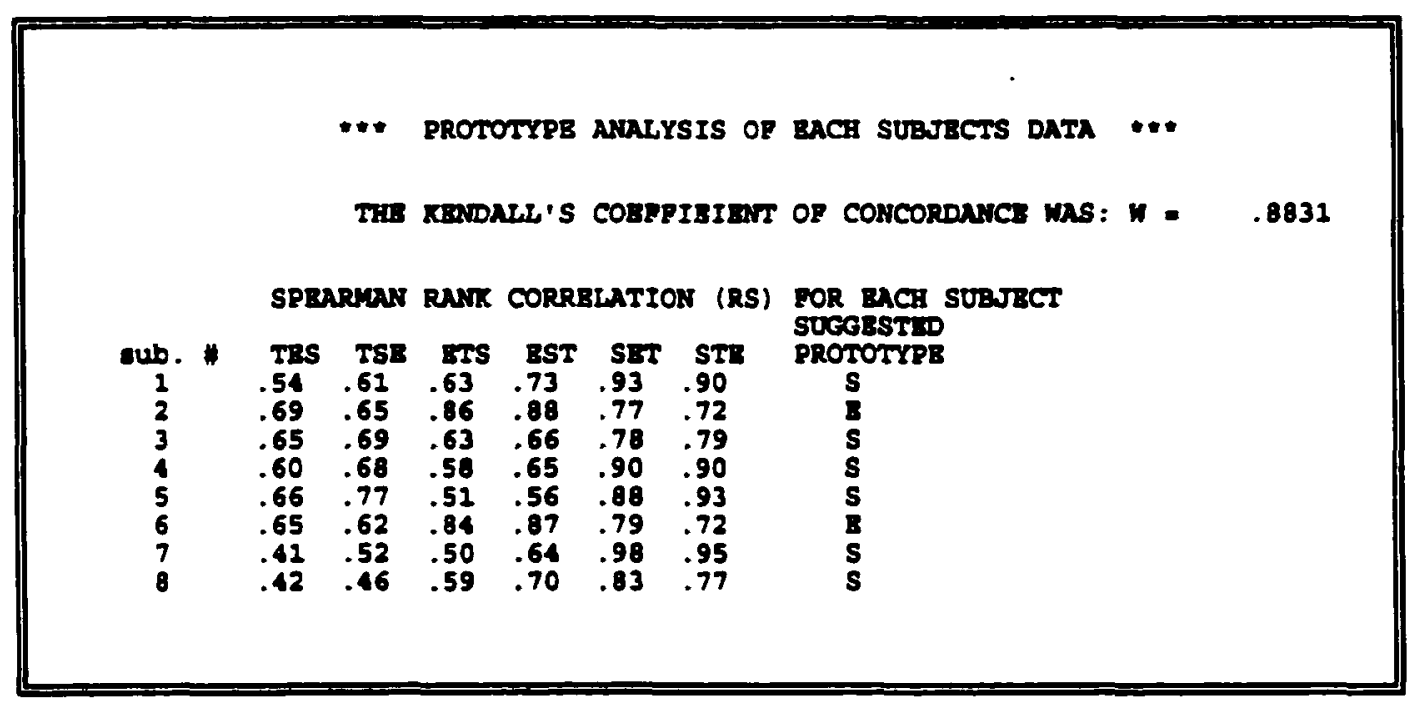

Figure 6.21: Goodness of Fit of Rescaled Values

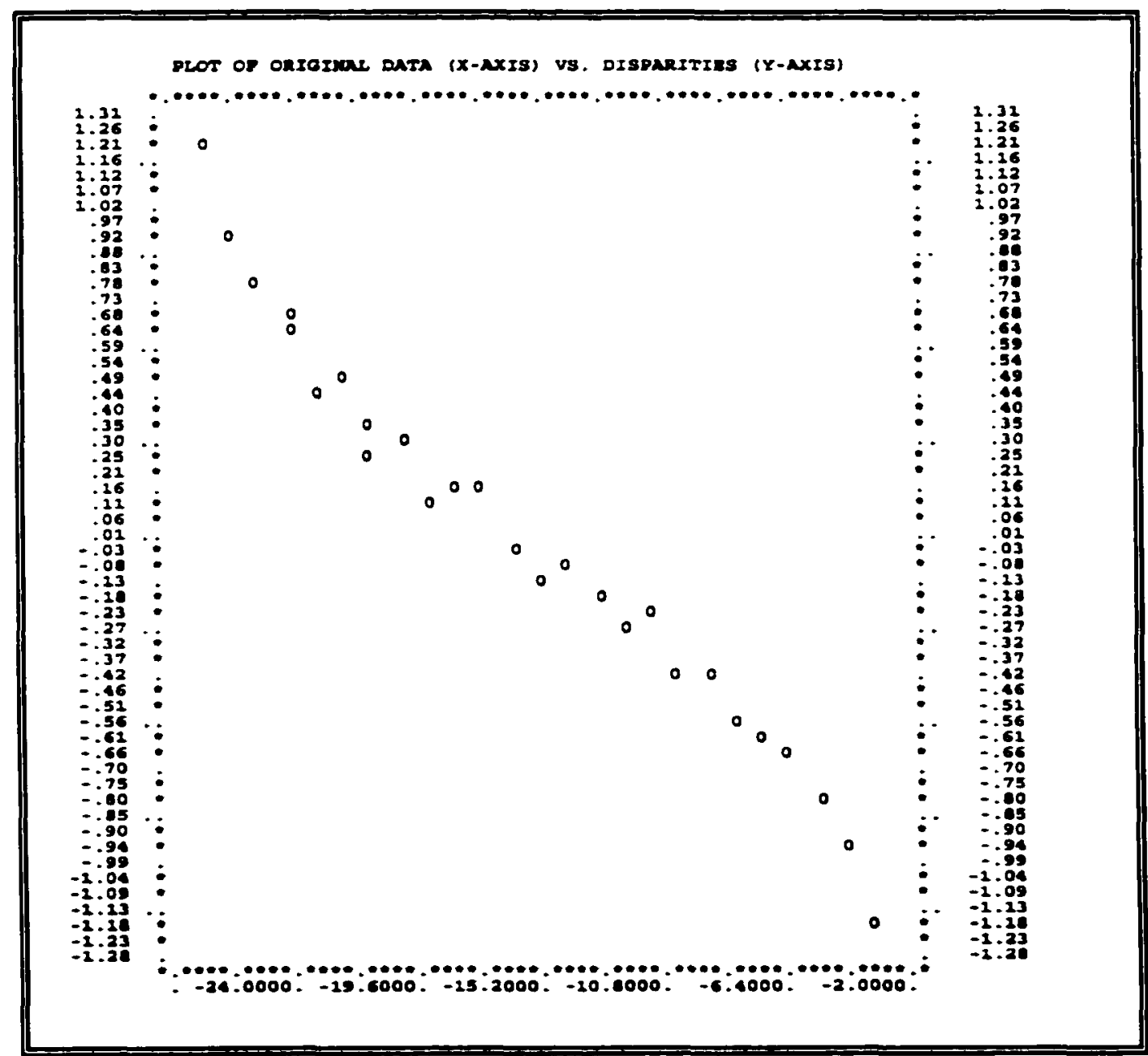


6.3.2.2 Event Scoring. The event scoring phase occurred immediately following each scenario. The scoring data for each participant was collected and collated so that the data could be analyzed later. The goals of data analysis were to:

1) Examine how the SWAT data agrees with the pre-assigned anomaly difficulty levels

2) Examine how the SWAT data agrees with the time data

3) Determine if there is natural delineation in the SWAT data so that task difficulty can be separated into three different levels (1,2 and 3$)$.

To have the data representation consistent with the analysis up to this point, it was decided that the rescaled SWAT values would be combined for the individual standard operators into a crew solution. This way, the four crews could be compared as in the time data analysis. Therefore, the event scoring was averaged into a single response for each anomaly event, resulting in four responses: expert-1, expert-2, standard-1 and standard-2.

First the data was plotted to see if there were any noticeable breaks in the data. The overlay plot in Figure 6.22 shows a slight delineation between the anomaly type difficulties, where the plot of 180 observations appears to show breaks at the 25 and 50 difficuity levels of the SWAT scale. One method of possible categorization is to refer to anomalies with a SWAT ranking of $\leq 25.0$ as Type $1, \leq 50$ as Type 2 , and $>50$ as Type 3 .

The purpose of this categorization is to generate a ranking method to guide automation of particular tasks. The intent is that automation might be easily performed on many of the Type 1 anomalies, and perhaps some of the Type 2 anomalies. Type 3 anomalies are generally not well suited for automating. 


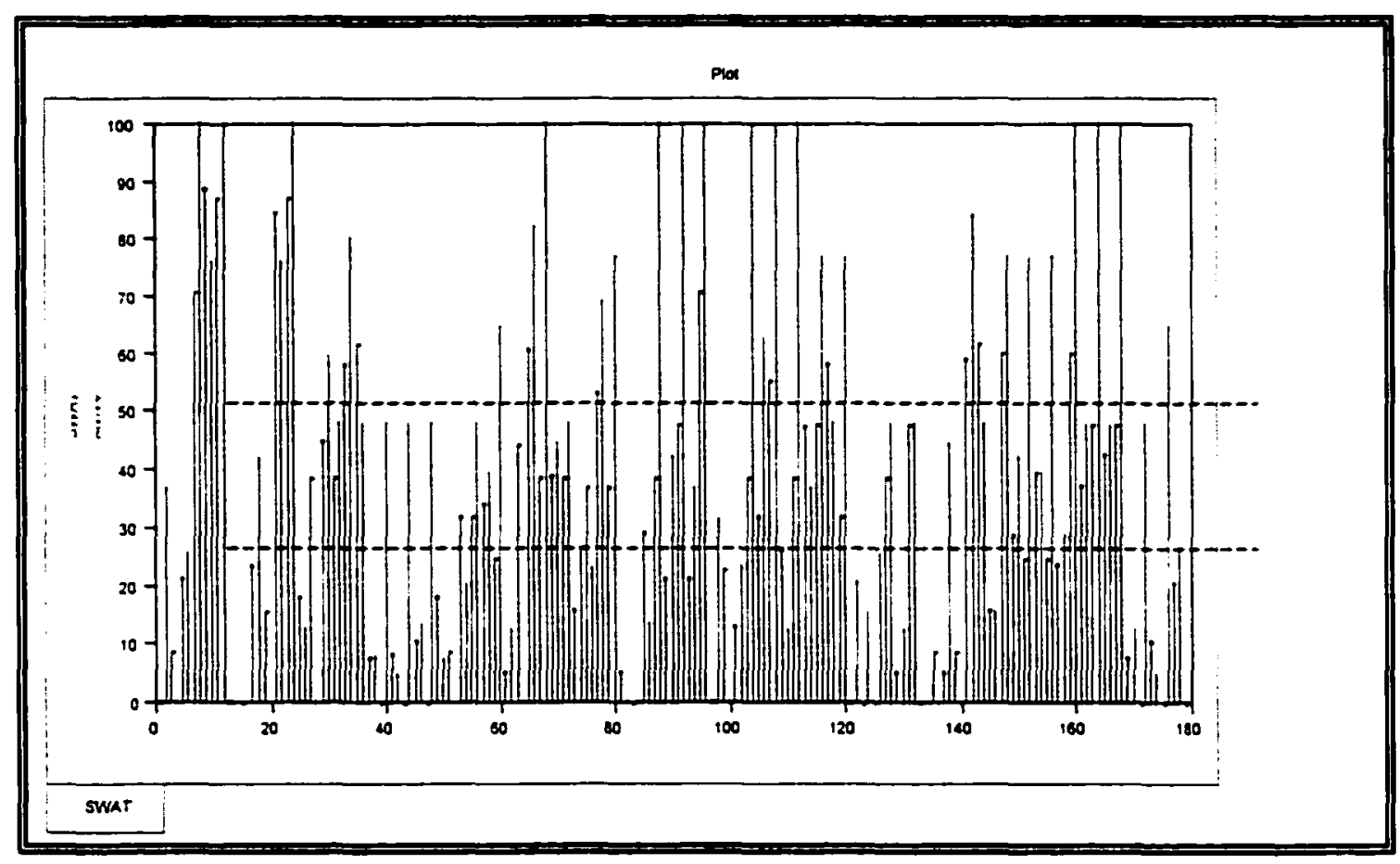

Figure 6.22: Overlay Plot of SWAT Anomaly Difficulty by resolution times

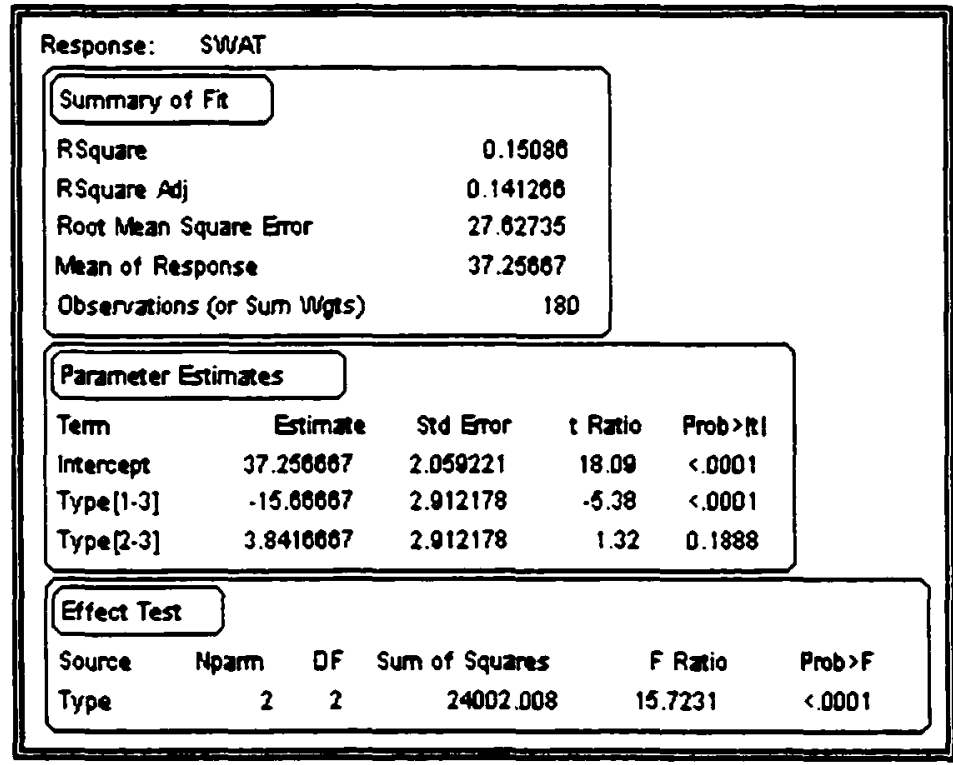

Figure 6.23: One-way analysis of variance, SWAT rating by type 
The analysis in Figure 6.23 compares the SWAT ratings by type. The high significance of type $(p=0.0001)$ shows that the initial type ratings are consistent with the perceived difficulty ratings assigned to each anomaly by the crews. The non-significant p-value for Type 2 and 3 comparisons reinforces the findings found in the Cox's Proportional Hazards analysis: the anomalies may have been either initially mistyped or the perceived difficulty between them was indistinguishable by the crews.

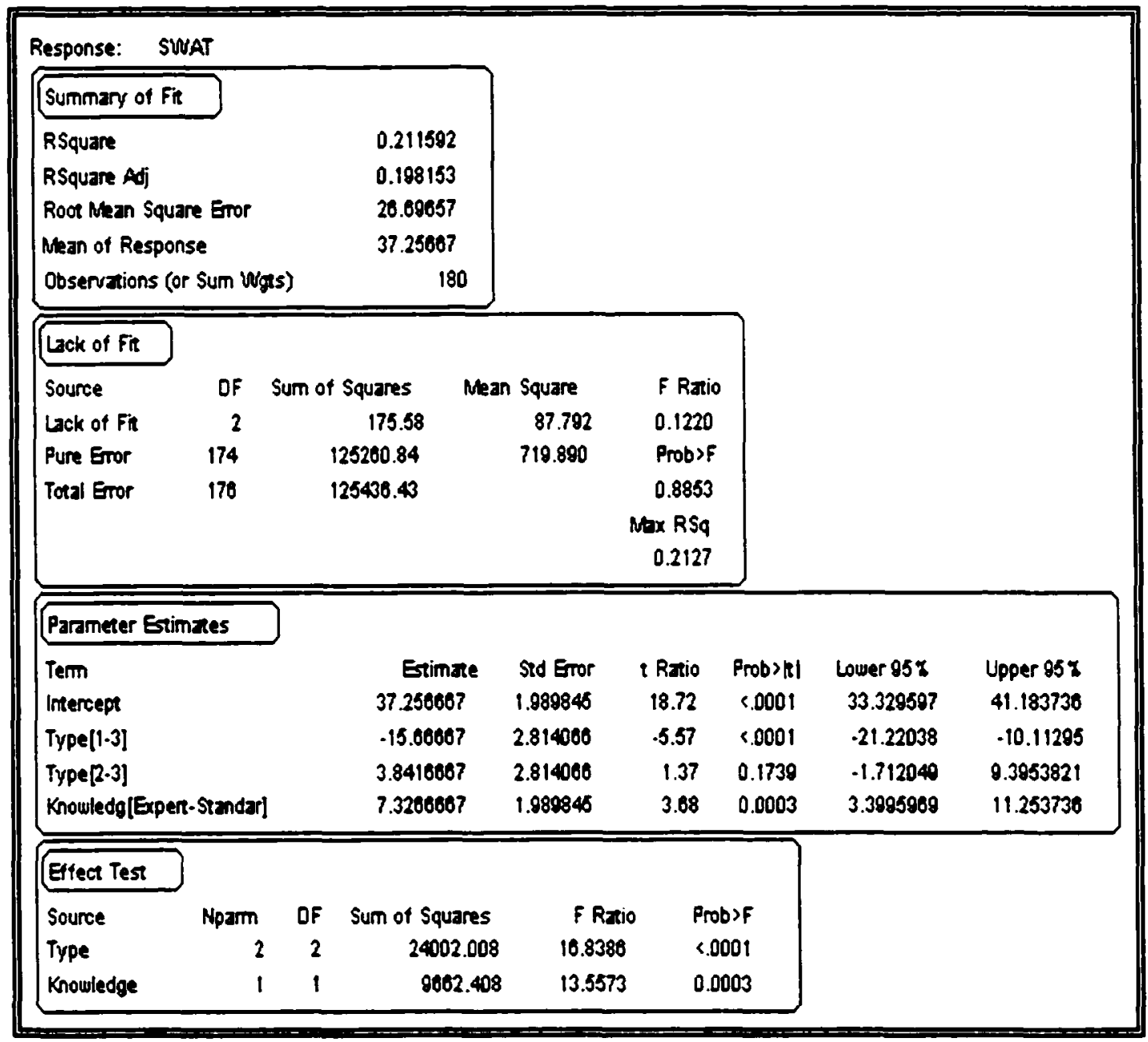

Figure 6.24: ANOVA with SWAT rating by type and knowledge 
The model in Figure 6.24 includes both type and knowledge in the analysis. The analysis of variance with two factors confirms the significance of type $(p=0.0001)$ and knowledge ( $p=0.0003$ ) in comparison with the SWAT ratings data.

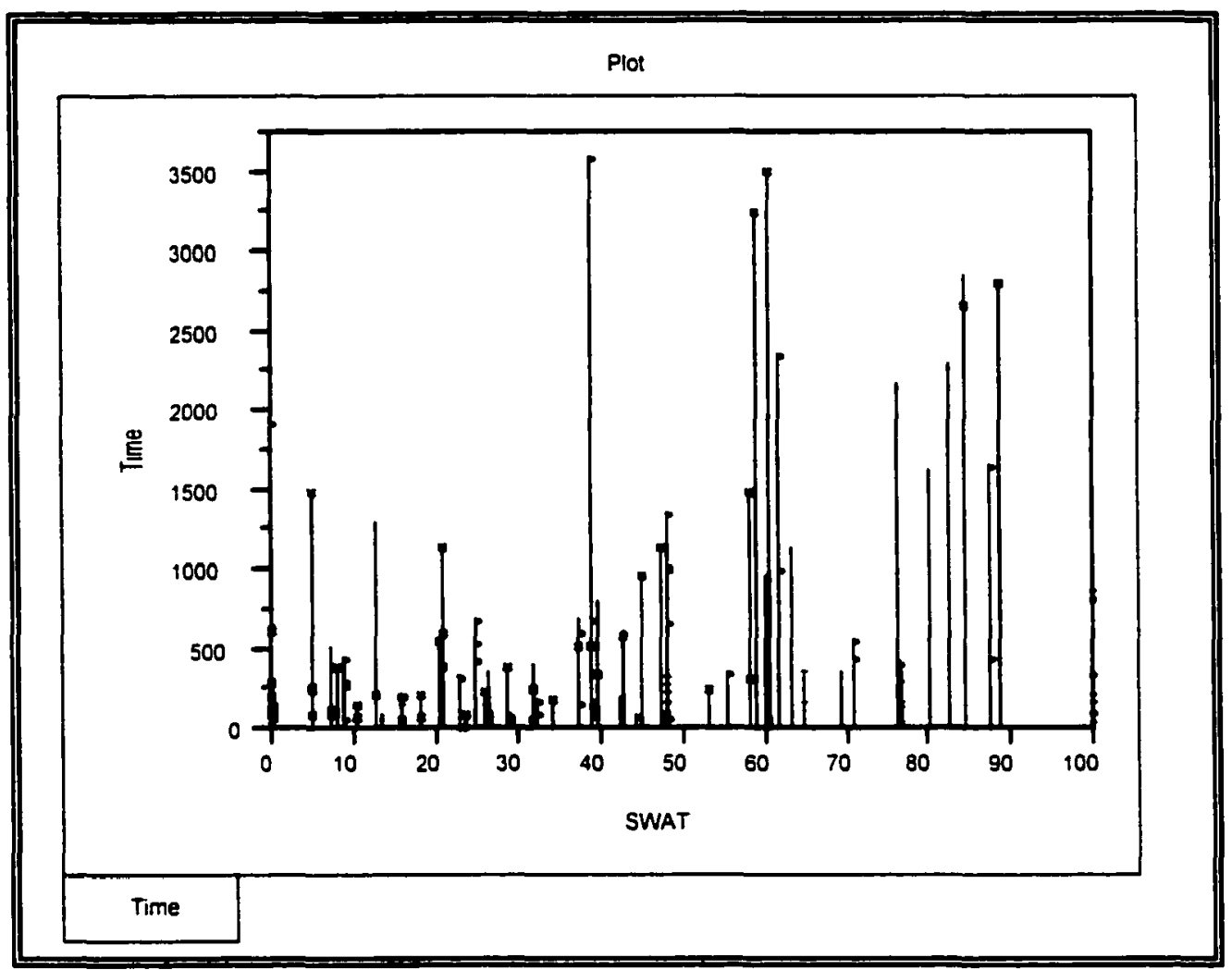

Figure 6.25: Resolution time by SWAT ratings

The plot in Figure 6.25 compares the resolution time with the perceived difficulty ratings. Note that for the most part, both values increase linearly. However, there are a few anomalies where the resolution time is large in comparison with the perceived difficulty level. This would indicate that the event, although requiring significant time to resolve, was not considered by the crew to be difficult. This also supports the fact that 
all subjects ranked Time Stress as the least significant of the three dimensions pertaining to perceived difficulty.

If the crews are further separated into standard and expert, this linearly increasing relationship is also confirmed by examining a scatterplot of time by SWAT rating, grouped by standard or expert (Figures 6.26 and 6.27 ). The lines fit for the groups show an overall increase of both time and SWAT rating. The ANOVA performed for each plot shows that the standard crew data for resolution time by SWAT rating was highly significant $\left(p_{\text {standard } 1}=p_{\text {standard } 2}=0.0001\right)$, showing evidence of high correlation between time data and SWAT ratings assigned to the anomalies. Expert's data only slightly agreed between resolution time and subjective difficulty ratings, showing only marginal significance $\left(p_{\text {exper } 1}=0.0692, p_{\text {exper } 2}=0.0868\right)$.

If the roles of SWAT rating and resolution time are switched, the results are virtually identical (Figures 6.28 and 6.29). Letting SWAT rating be the response, and resolution time be the predictor (since it is measured precisely), the standard crew pvalues were identical to the analysis in $6.24\left(p_{\text {standard } 1}=p_{\text {standard } 2}=0.0001\right)$. Expert's results were also the same $\left(p_{\text {expert } 1}=0.0692, p_{\text {expert } 2}=0.0868\right)$.

An analysis of covariance (ANCOVA) was performed with SWAT rating as the response, and resolution time as a continuous predictor, containing factors of knowledge, type and knowledge*type. The initial analysis showed that the interaction term was not significant $(p=0.929)$, and was eliminated from subsequent runs. The final ANCOVA may be seen in Figure 6.30. All factors included were highly significant $\left(p_{\text {time }}=0.0008, p_{\text {knowledge }}=p_{\text {type }}<0.0001\right)$. In sum, this alpha-level significance demonstrates that anomaly typing cannot be based on resolution time alone. 


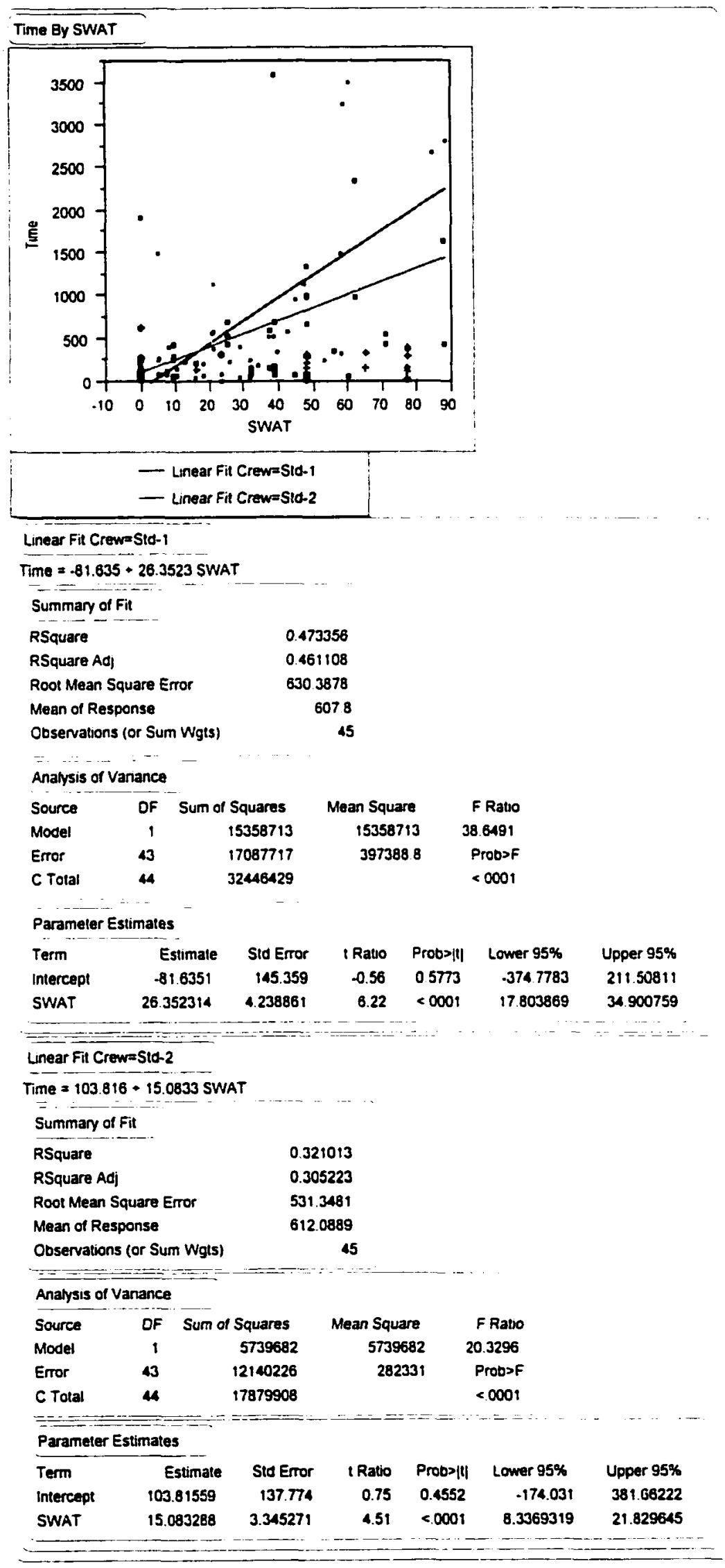

Figure 6.26: Scatterplot of resolution time by SWAT ratings for standard crews 


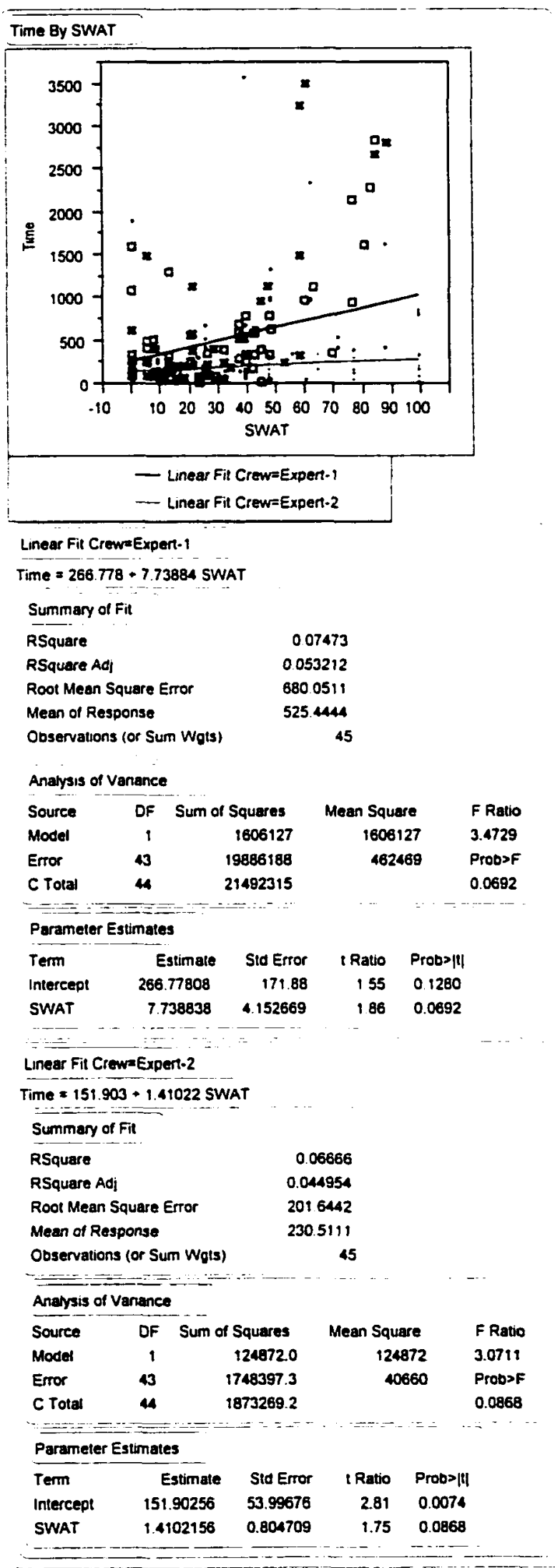

Figure 6.27: Scatterplot of resolution time by SWAT ratings for expert crews 


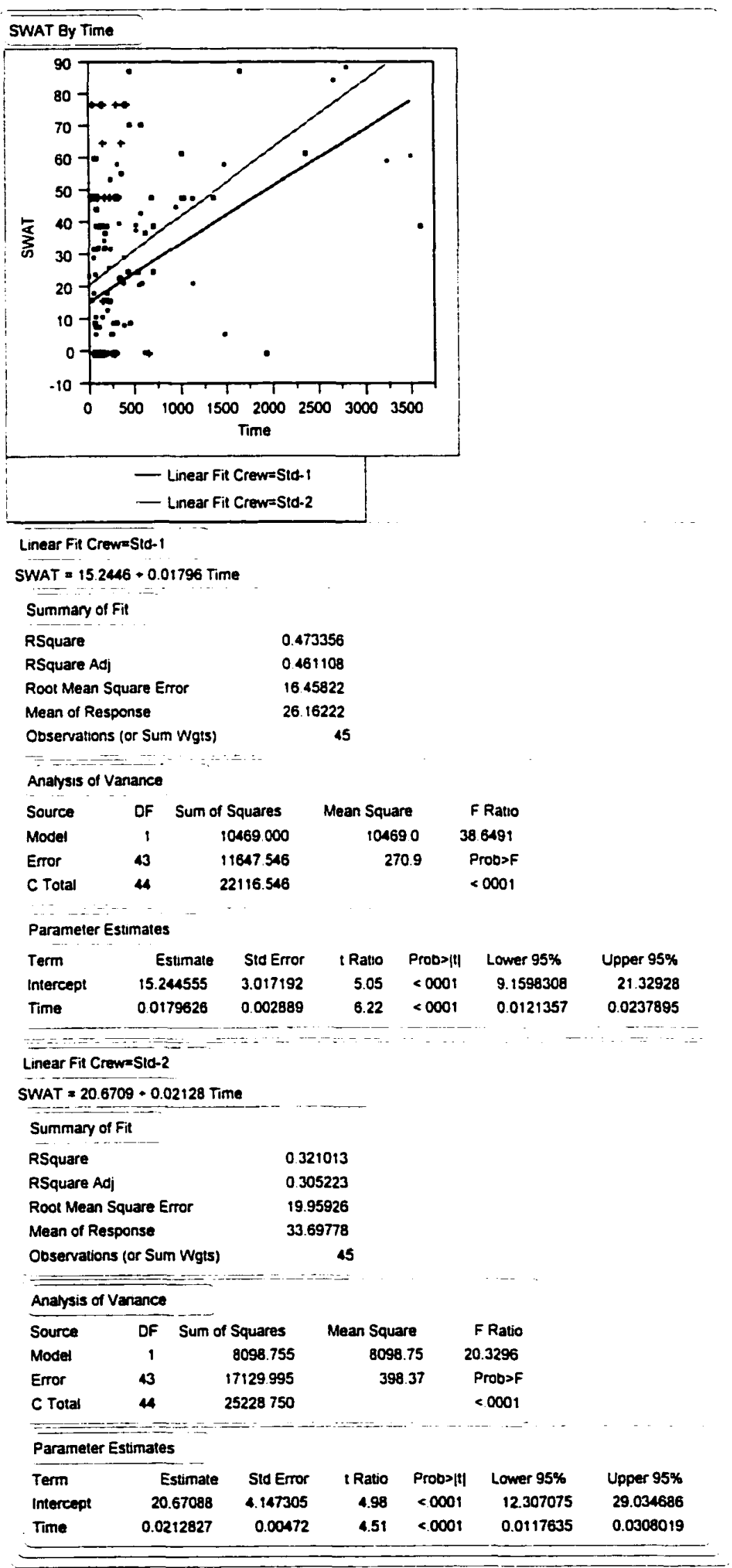

Figure 6.28: Scatterplot of SWAT ratings by resolution time for standard crews 


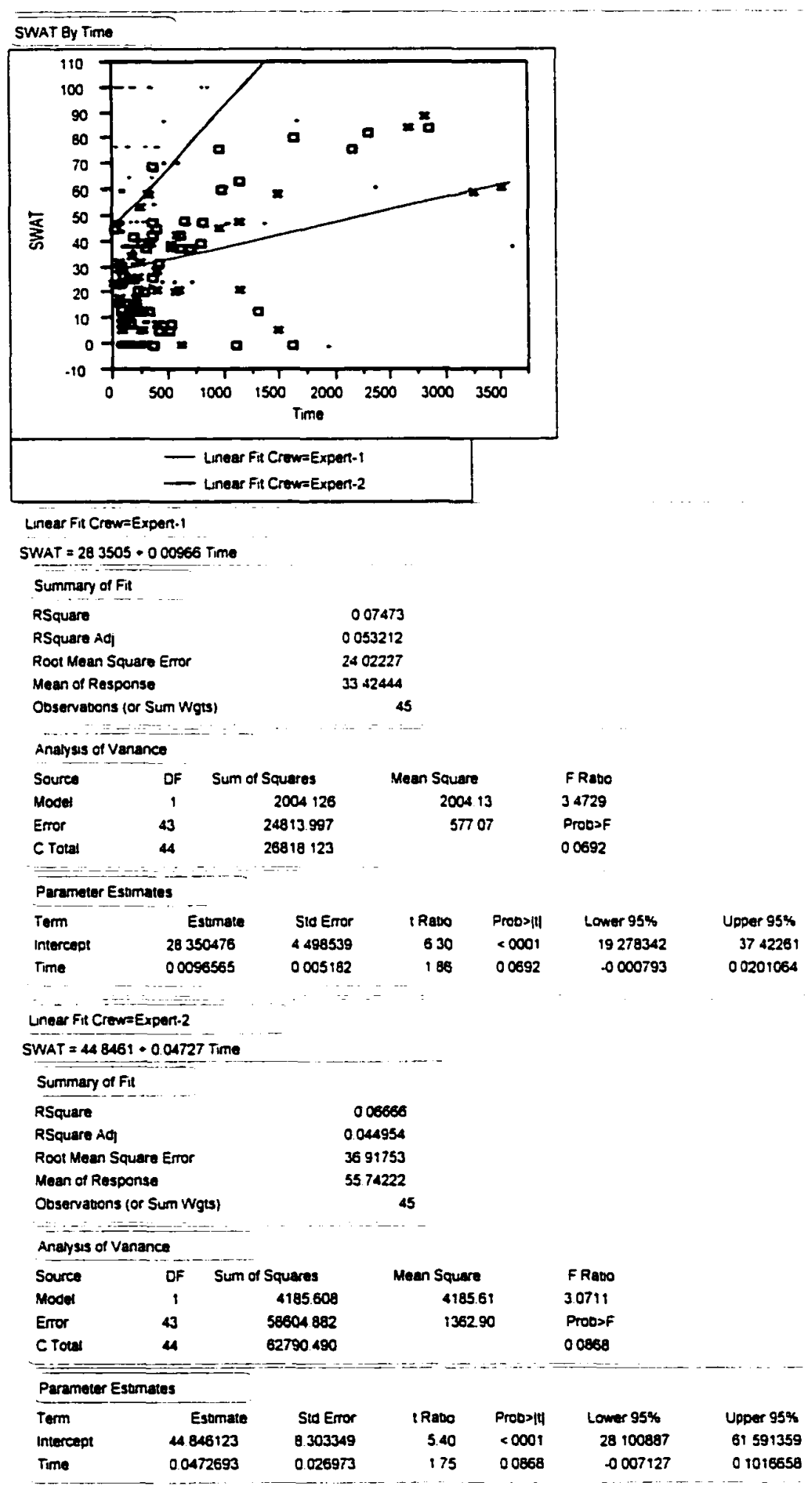

Figure 6.29: Scatterplot of SWAT ratings by resolution time for expert crews 


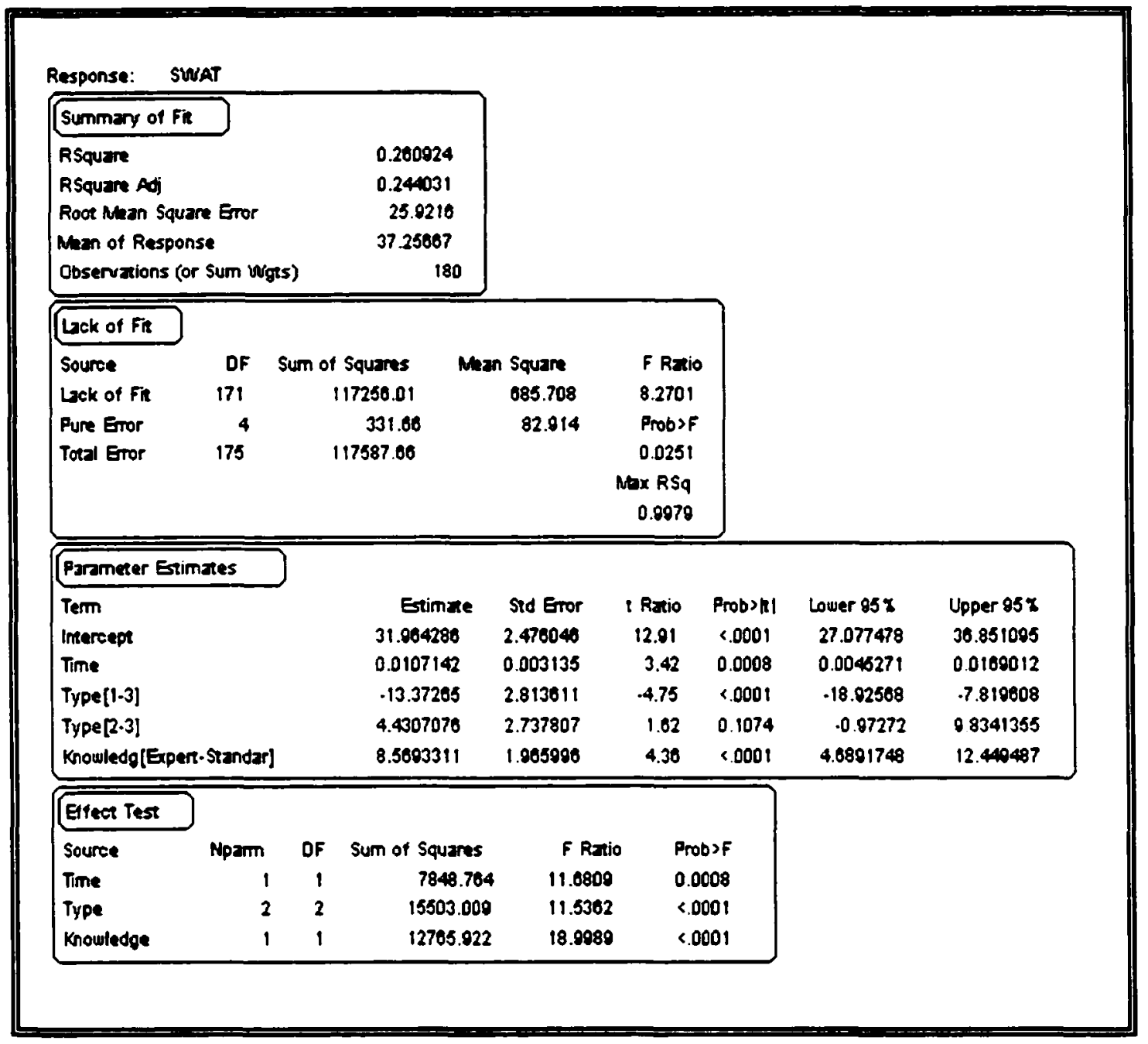

Figure 6.30: ANCOVA of SWAT ratings by resolution time, knowledge and type 
Correlation between perceived difficulty and resolution time for each of the four crews were also examined (Figure 6.31). Correlation is a measure of the strength of association between two variables. There is a higher correlation between the SWAT data and the time data for the standard crews, while the experts show much weaker correlation, suggesting that there is greater connection between perceived difficulty and time to resolve for the standard crews. This table also includes the covariance matrices.

\begin{tabular}{|c|c|c|c|c|c|}
\hline $\begin{array}{l}\text { Crewraxpert-1 } \\
\text { Multivariate }\end{array}$ & & & $\begin{array}{l}\text { Crewrestandard-1 } \\
\text { Multivariate }\end{array}$ & & \\
\hline $\begin{array}{l}\text { Correlations } \\
\text { SWAT } \\
\text { Time }\end{array}$ & $\begin{array}{l}\text { SWAT } \\
1.0000 \\
0.2734\end{array}$ & $\begin{array}{r}\text { Time } \\
0.2734 \\
1.0000\end{array}$ & $\begin{array}{l}\text { Correlations } \\
\text { SWAT } \\
\text { Time }\end{array}$ & $\begin{array}{l}\text { SWAT } \\
1.0000 \\
0.6880\end{array}$ & $\begin{array}{r}\text { Time } \\
0.6880 \\
1.0000\end{array}$ \\
\hline $\begin{array}{l}\text { Covariance Matrix } \\
\text { SWAT } \\
\text { Time }\end{array}$ & $\begin{array}{r}\text { SWAT } \\
609.50280 \\
4716.8434\end{array}$ & $\begin{array}{r}\text { Time } \\
4716.8434 \\
488461.71\end{array}$ & $\begin{array}{l}\text { Covariance Matrix } \\
\text { SWAT } \\
\text { Time }\end{array}$ & $\begin{array}{r}\text { SWAT } \\
502.64877 \\
13245.958\end{array}$ & $\begin{array}{r}\text { Time } \\
13245.958 \\
737418.85\end{array}$ \\
\hline $\begin{array}{l}\text { CrewaExpert-2 } \\
\text { Multivariate }\end{array}$ & & & $\begin{array}{l}\text { Crewestandard-2 } \\
\text { Multivariate }\end{array}$ & & \\
\hline $\begin{array}{l}\text { Correlations } \\
\text { SWAT } \\
\text { Time }\end{array}$ & $\begin{array}{l}\text { SWAT } \\
1.0000 \\
0.2582\end{array}$ & $\begin{array}{r}\text { Time } \\
0.2582 \\
1.0000\end{array}$ & $\begin{array}{l}\text { Correlations } \\
\text { SWAT } \\
\text { Time }\end{array}$ & $\begin{array}{l}\text { SWAT } \\
1.0000 \\
0.5666\end{array}$ & $\begin{array}{r}\text { Time } \\
0.5666 \\
1.0000\end{array}$ \\
\hline $\begin{array}{l}\text { Covariance Matrix } \\
\text { SWAT } \\
\text { Time }\end{array}$ & $\begin{array}{r}\text { SWAT } \\
1427.0566 \\
2012.4575\end{array}$ & $\begin{array}{r}\text { Time } \\
2012.4575 \\
42574.301\end{array}$ & Covariance Matrix & $\begin{array}{r}\text { SWAT } \\
573.38068 \\
8648.4661\end{array}$ & $\begin{array}{r}\text { Time } \\
8648.4661 \\
406361.54\end{array}$ \\
\hline
\end{tabular}

Figure 6.31: Correlation and covariance matrices of SWAT ratings by resolution time for expert and standard crews

\subsection{Summary}

The time data presents convincing evidence that there is a considerable difference in resolution times between the expert and standard knowledge levels. Knowledge main effects were significant at a p-value of 0.0016 . This verifies there was 
a significant difference in resolution ability between knowledge groups, and the hazard functions were not identical $(R R=1.274, R R C l[1.095,1.482])$. This would lead to the rejection of the null hypothesis that there is no difference in expert or standard groups' time to resolve.

The main effect of anomaly type shows a highly significant Effect Likelihood Ratio Test of $p \approx 0$, indicating that the null hypothesis of anomaly resolution times being identical across different anomaly types should be rejected.

The appropriate SWAT scaling solution was a group solution, proving the operators were in general agreement with how they defined perceived difficulty and how they ranked the three dimensions of the time effort $(T)$, mental effort $(E)$, and psychological stress (S) ratings.

Analyses confirm that whether the SWAT data is modeled the predictor or the response, type is a significant effect when modeled with resolution time. The high correlation indicates that resolution time alone cannot be the sole consideration when performing anomaly typing for automation 


\section{Chapter 7}

\section{DISCUSSION/RECOMMENDATIONS}

\subsection{Discussion}

\subsubsection{Time Data.}

Operators resolved Type 1 anomalies 1.680 times faster on average than Type 3 anomalies. Type 2 anomalies were resolved in roughly the same amount of time as Type 3's $(R R=0.984 \equiv 1, R R C I[0.792,1.213])$, indicating that the difficulty level between the two types is similar among all operators, or that the initial differentiation between anomaly difficulties of Types 2 and 3 was inaccurate. In this instance, subjective data collected during the experiment will prove to be extremely useful, as anomaly difficulty should not be represented solely by resolution time.

The time data presents convincing evidence that there is a considerable difference in resolution times between the expert and standard knowledge levels. Knowledge main effects were significant at a p-value of 0.0016 . This verifies there was a significant difference in resolution ability between knowledge groups, and the hazard functions were not identical $(R R=1.274, \operatorname{RRCl}[1.095,1.482])$. This would lead to the rejection of the null hypothesis that there is no difference in expert or standard groups' time to resolve.

Experts were 1.274 times faster overall (RRCl [1.095, 1.482]) in resolving anomalies than the standard group. Experts were also 2.140 times faster in resolving Type 1 anomalies than Type 3's, whereas standard crews were 1.680 times faster in resolving Type 1's than Type 3's. However, for Type 2 anomaly resolution, again there 
was not a noted difference between that of resolving Type 2's and 3's.

The data showed that there was a significant difference in ability to resolve anomalies between expert and standard groups. Although there was some variation in the ability levels of the expent crews, overall they outperformed their standard counterparts ( $p$ value of 0.0016 ). In a single time period, experts could resolve 1.28 more anomalies than standard crews.

The main effect of anomaly type shows a highly significant Effect Likelihood Ratio Test of $p \approx 0$, indicating that the null hypothesis of anomaly resolution times being identical across different anomaly types should be rejected. More specifically, there was a significant difference in overall operator ability in resolving Type 1 anomalies over Types 2 or 3: Type 1 anomalies were resolved 1.69 times faster than Type 3 anomalies. There was no evidence of significant difference between Type 2 and 3 resolution times, as they were resolved in roughly the same amount of time. This could be attributed to not appropriately differentiating between the types during initial classification.

There was definite variation in the expert crew knowledge levels, demonstrating that one expert (expert 2) is much more consistent in resolution ability than the other expert and both the standard crews.

\subsubsection{Subjective Data.}

The appropriate SWAT scaling solution was a group solution, proving the operators were in general agreement with how they defined perceived difficulty and how they ranked the three dimensions of the Time Effort (T), Mental Effort (E), and Psychological Stress (S) ratings. The Subjective Workload Analysis Technique (SWAT) analysis demonstrated that the crews felt that Psychological Stress (S) was the most important dimension in determining subjective workload. The Kendall's Coefficient of Concordance for the study data was $0.883(83.3 \%)$, indicating a high correlation 
between how the subjects defined "difficulty". Six out of eight participants thought that Psychological Stress was the most appropriate descriptor of effort. Two of the eight subjects (subjects 2 and 6) thought that Mental Effort was the most significant dimension; however, the overall correlation among the group was high enough so that they aiso matched the Psychological Stress prototype. This leads to the conclusion that it would be inappropriate to use resolution time as the sole measure of operator ability, since Time Stress (T) was rated as the lowest dimension.

There appeared a natural delineation in the raw data at the SWAT Ratings of 25 and 50, leading to possible categorization levels for type segregation. Natural breaks in the data can help guide automation, enabling automation of Type 1 anomalies and possibly some Type 2 tasks. Discretion must be used when selecting candidates for automation by choosing tasks that are more easily automated, have highly deterministic indicators, and are unaffected by software/hardware limitations.

Analyses confirmed that whether the SWAT data is modeled as the predictor or the response, type is a significant effect when modeled with resolution time. The high correlation indicates that resolution time alone cannot be the sole consideration when performing anomaly typing for automation.

Standard crew data for resolution time by SWAT rating was highly significant $\left(p_{\text {standard } 1}=p_{\text {standard } 2}=0.0001\right)$, showing evidence of high correlation between time data and SWAT ratings assigned to the anomalies. Expert's data only slightly agreed between resolution time and subjective difficulty ratings, showing only marginal significance ( $p_{\text {expen } 1}=0.0692$, $p_{\text {exper } 2}=0.0868$ ). There was also evidence of high significance between pre-specified anomaly type and SWAT difficulty rating $(p=0.0001)$.

The bottom line is that automation typing may not be accurately performed on resolution time alone. Consideration of subjective data (perceived difficulty) should be given when separating tasks into different difficulty levels. 


\subsubsection{Additional Analysis and Considerations}

The anomalies were initially grouped by difficulty Types 1,2 and 3. This was based on operator experience and exposure, historical probability of occurrence, likelihood, intensity of resolution process and estimated level of training required for resolution. Although these were "educated" categorizations, the data showed minimal discrimination of resolution ability between Type 2 and 3 anomalies. Therefore, it seemed advantageous to analyze the data separated into two difficulty levels: Type 1 and Type $2 / 3$.

The analysis results demonstrated that although it initially seemed beneficial to classify the data into two types, this resulted in an unbalanced design. In the "combined" design, there was a total of 60 Type 1 observations and 120 combined Type $2 / 3$ 's. The results still gave highly significant $p$-values, but the unbalanced design slightly skewed the risk ratio values. Therefore, a more ideal solution would be either to 1) re-accomplish the experiment with only two anomaly types and ensure a balanced design with equal anomalies, or 2) randomly sample the Type $2 / 3$ observations to include only 60 of the observations in the design. Regardless, a meaningful combined data analysis could only be facilitated through experiment redesign.

One notable point to consider is the six censored observations. These observations resulted from the crew being unable, either due to confusion or lack of training and experience, to resolve the anomaly. An important question to contemplate is the following: are these data points significant enough to propel the results to another level? Specifically, if the operator is trained at a certain level, could one conclude that at that particular level of experience and training that they would never be able to resolve that anomaly? It is possible or reasonable to suggest that since they were never exposed to the "answer", they might never arrive confidently at that correct answer. 
More importantly, can that conclusion be drawn based on the data presented here alone? Regardless of the ability or inability to prove this hypothesis, this leads to a puzzling and intriguing problem worthy of further investigation.

\subsection{Recommendations}

The intent of this research was to provide data to aid in investigating the role of human knowledge in the combined human-machine unit. The results lead to the possibilities of:

1) decreasing the number of human operators

2) increasing human knowledge level (experience and training)

3) applying this derived information and knowledge in developing a new, improved machine-system capability through future research.

The following are outcomes or recommendations based on the study results:

7.2.1 Operators. One expert operator can surpass a crew of four standard operators' anomaly resolution capability in terms of accuracy and efficiency. Although the reduction in crew size is an end result, the principal finding is to enhance the level of training and experience of the operator crew force. This can be accomplished while simultaneously reducing the number of personnel needed. This is in agreement with the current military philosophy: a tendency towards reduction in crew sizes while guarding against redundancy compromises.

7.2.2 Continuity. Maximize "knowledge" by keeping personnel in the job longer. It appears that the current time on station (approximately three years) is not adequate to achieve the level of training and experience needed for operators to be able to effectively resolve anomalies above the Type 1 difficulty level. Adequate continuity is 
the only way to achieve increased operator experience, an indispensable part of the expert operator composition. A higher degree of continuity could be achieved by several means:

1) Extending time on station requirements for military operator personnel

2) Transition satellite operations to a Air Force Reserve crew force who can remain in place indefinitely

3) Migrate towards a DoD civilian or contractor crew with military oversight

7.2.3 Training. Train personnel for longer periods of time (apprenticeship programs). Create operator redundancy by training multiple operators so that there is overlap for satellite crises, employee vacations and employee turnover. Instruct operators to an "engineering" level of knowledge; in greater depth than the current standard. Opposing this recommendation is the current military trend towards skill level reduction, or transition of positions to the lowest pay grade to reduce costs. However, the study data would indicate that this technique reduces the anomaly resolution ability resident within the crew force.

7.2.4 Task difficulty and awareness. When assessing task difficulty, resolution time is an effective measurand. However, gauging perceived difficulty is necessary to determine true difficulty level and evaluate what tasks are well suited for automation. Active promotion of task surveys could determine what operator actions are candidates for automation. This would aid in defining tasks that could be removed from manual resolution so that operator attention could be directed to more non-deterministic problems. It could also help in training issues, where constant definition and revision of 
typical and atypical performance tasks are necessary in designing complete and effective training programs.

7.2.5 Personnel Cost Estimates. One standard crew currently costs more to maintain than a crew of one expert operator. Cost is a major factor when determining what person should perform the job. The current philosophy is to train brand new recruits to perform satellite operations. The rationale behind this method is to reduce labor costs, as enlisted operators are much less costly than paying officers to perform the job. However, as seen in the table below, one standard crew can cost significantly more to maintain than a crew of one expert operator.

\begin{tabular}{|c|c|c|c|}
\hline Position & Pay Grade & $\begin{array}{c}\text { Estimated } \\
\text { Average Annual } \\
\text { Income * }\end{array}$ & $\begin{array}{c}\text { Cost for One } \\
\text { Crew }\end{array}$ \\
SSO & E1-E3 & $\$ 19,647.60$ & \\
SSO-2 & E1-E3 & $\$ 19,647.60$ & \\
ENG & O3-E5 & $\$ 22,595.40$ & $\$ 102,369.40$ \\
\hline EXPERT & N/A & $\$ 40,474.80$ & \\
\hline
\end{tabular}

Figure 7.1: Estimated crew costs

Experts, being in a training phase for considerably longer than their standard counterparts, would require some type of apprenticeship (formal school and on-the-job training) phase. Therefore, it seemed prudent to base estimates of one "expert" crew as a crew with one fully qualified expert plus an expert apprentice-in-training. This training 
phase could last 3 years. Conversely, the standard crews might require apprenticeships of only a few months. What this implies is the following: standard crews are "ready" to perform the job at the standard level a much earlier time, while experts (in order to achieve the "expert" knowledge level), would not be able to operate autonomously for a much longer period. However, it is important to consider that even if the standard crews are deemed crew qualified faster, this does not negate the additional cost of on-site contractor support. The on-site contractor team is currently comprised of approximately 8 individuals. The argument is that if expert operators are trained at the desired level of proficiency, the necessity for contractor support on-site would be greatly diminished, and possibly unnecessary.

If an ample crew force is developed, situations of operator turnover, crew leave/vacation, and sick time must be considered. It was estimated that six crews could adequately fill all shifts while still leaving sufficient personnel to allow for excess coverage. The following estimates are based on $24 / 7$ operations, 8 hour shifts.

\begin{tabular}{|c|c|c|}
\hline \multicolumn{1}{|c|}{ Crew } & $\begin{array}{c}\text { Number of } \\
\text { Crews/Operators }\end{array}$ & Estimated Annual Cost \\
\hline Standard & 6 Crews (24 Operators) & $\$ 614,216.40$ \\
Expert & 6 Crews (12 Operators) & $\$ 420,000.00$ \\
\hline
\end{tabular}

Figure 7.2: Estimated crew force and associated costs

In short, it is obvious that even though a single expert operator costs more than a standard operator, the overall crew costs should be considerably less. To make this option even more appealing, an expert crew has significantly more ability and knowledge than the standard crews, and should provide higher return for the investment. 
7.2.6 Screen Re-design. In consideration of the findings presented in Chapter 6, this section offers some considerations for the next step in the system design process. Research conclusions stated thus far focus only on varying operator knowledge levels and crew force. However, a follow-on study should be conducted in the future to examine the benefit gained through display redesign and technology improvement. This portion investigates potential solutions for technical and machine issues associated with system design. The Center for Research Support (CERES) plans to examine the machine component of the system formally following their assessment of the data presented in this study.

7.2.6.1 Background. The current screens by which data is viewed are cumbersome, hard to read, and over saturates the operators with unnecessary data. According to Elvers, et al. (1993), "the role of the human in many complex systems is as a system monitor. Rather than providing a single overall indication of their status, these systems tend to present the operator with numerous separate pieces of information, none of which reflects overall status." Furthermore, Jones et al. (1995) verifies that a hierarchical structure applied to data display (in this particular example, it was applied to satellite telemetry data) can be useful in managing complexity.

After detailing the purpose of satellite telemetry displays and standard crew tasks, a preliminary exploration of potential display schemes will be presented in this section. An important note is that screen redesign will not affect other users extensively in the network. Satellite telemetry screens are used exclusively by the 1 SOPS operators and their backup node, and screen displays are generated by interpreting the existing telemetry streams emanating from the vehicle. Altering the interpretation of these streams does not affect the Automated Remote Tracking Station, satellite vehicle or end-users of the data. 
7.2.6.2 Satellite State-of-Health. The state-of-health screens are not user friendly and saturate the operator with unnecessary data. An example of the satellite bus telemetry frame may be viewed in Figure 7.3. Over 650 data points are displayed to the operator through several screens -- many are repeated values. It is not to say that the data on the screens is not useful. However, for the typical "Type I anomalies" that are easily fixed, it is not always necessary to examine the data to the level of detail presented regularly to the operator. As previously noted, the satellite State of Health (SOH) process involves a series of approximately seven computer displays that present the data in either color-coded mnemonics (representing various subsystem entities, such as battery temperatures, etc.), telemetry patterns, or pure numerical values. Certain ranges are specified, and if the point is determined by the system to be "out of limits" because it is out of range, the mnemonic appears yellow (warning) or red (critical). Another data check is a manual check of numerical data, consisting of an entire page requiring an actual value check. This methodology greatly multiplies the risk of human error. The claim is that these functions are well suited for computers, but not for humans. Additionally, the number of screens can be drastically reduced by at least half. In our redesigned system, the only data necessary for display are out-of-limit points; points within limits are considered nominal and should be eliminated from the initial display. This will greatly minimize the time and personnel required for routine, nominal SOH's.

7.2.6.3 SOH Screens. The redesign involves a combination of audio/video notifications involving anomalies. It is a hierarchical display, originating from simplistic to complex. The operator has a view of the entire satellite constellation (a change from the current method of control, where the crew examined the SOH on only one satellite at a time) and gets immediate feedback of an anomaly off the main screen. Each satellite 
monitored will show green in the absence of anomaly. Upon a change in status, the box will change color (either yellow or red, indicating severity of the problem) and will be accompanied by an audible alarm. The operator can 'mouse click' on the affected vehicle, bringing up a series of menus arranged according to subsystem. The affected subsystem(s) will also have the color displayed on the initial box, so as to guide the operator through the correct path. Simultaneously, if the problem is a Type 1 or 2 anomaly, the suggested plan of action (in terms of anomaly commanding) will present itself in a separate box, allowing the operator to execute the plan if necessary. Figures 7.4 through 7.8 display the new screen designs for the SOH process.

Currently, military operators are required to have "full health". Primarily, crew members have full corrected visual acuity, and must be $\leq 20 / 20$ (corrected). Vision is tested annually, and space operators cannot be colorblind. If this requirement was to be waived so colorblind individuals were not excluded, there are some possible substitutions for color processing and alarms. Some possible replacement schemes are indicating anomalous conditions or alarms via blinking subsystem components, expanding three-dimensional alarm blocks, varied font, and similar techniques.

7.2.6.4 Satellite Commanding. In the area of satellite commanding, command plan checklists exist for several possible anomalies or system reconfigurations. The current command plans involve the human manually typing in each command to the satellite. Figure 7.8 shows one possible command window scheme. It becomes immediately obvious that there is currently great potential for error - the incorrect command sent at an inopportune time can have disastrous effects on the vehicle. 


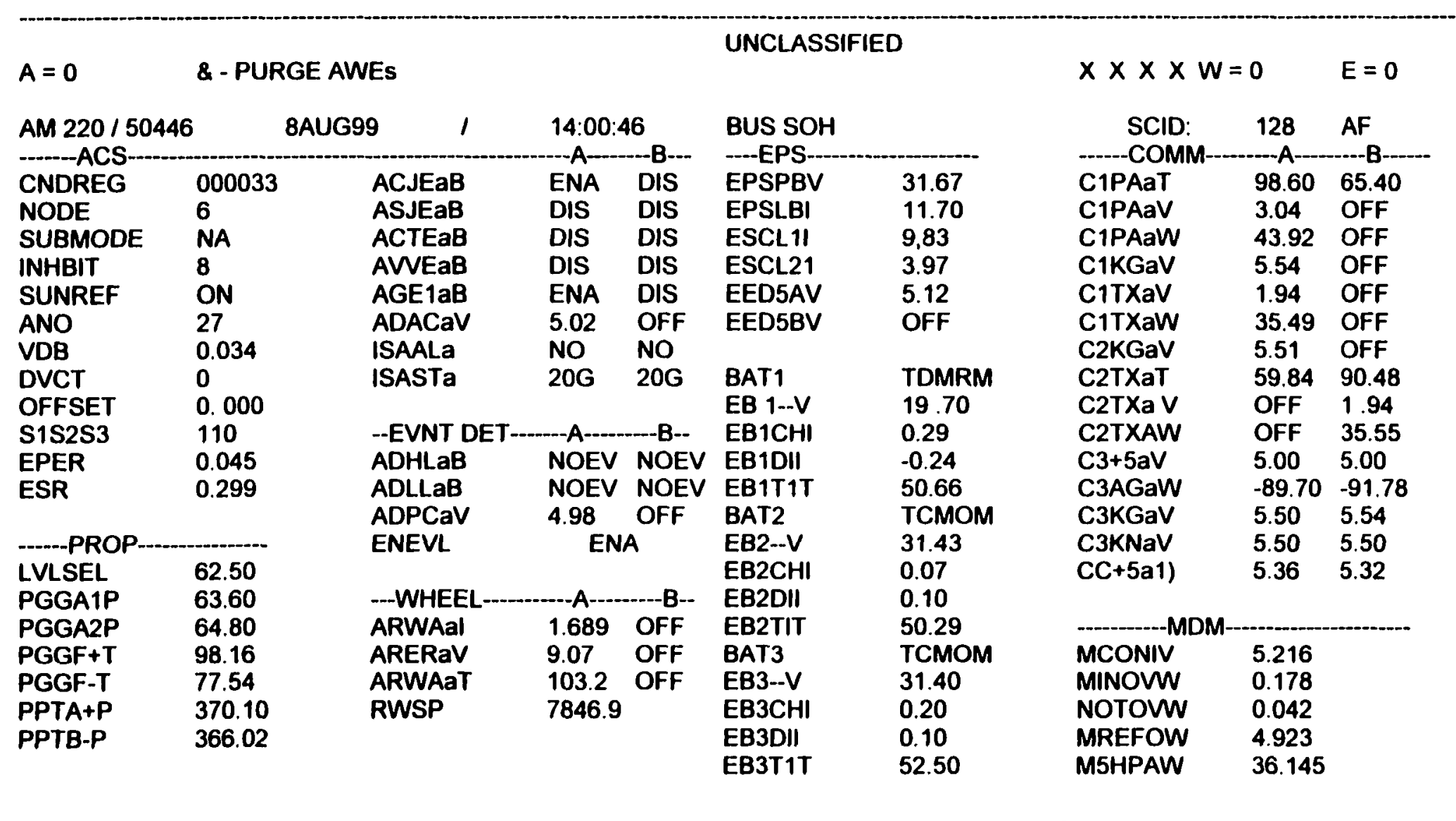

\& - PURGE AWES

UNCLASSIFIED

Figure 7.3: $128 \mathrm{~K}$ Telemetry - BUS SOH (Current System) 
In addition, not all necessary command sequences exist in checklist format, and the operators (at the current knowledge level) are unable to act promptly if any of the lessprobable conditions exist. Therefore, it is advantageous to have all known command sequences loaded and/or automated so that timeliness is maximized and errors are minimized. It is important to bear in mind that automation is no substitute for knowledge level, and this is most apparent when uncertainty enters the scenario. Uncertainty makes determining the correct command plan for execution a challenge -- a responsibility that at this time cannot be relinquished to a machine. This is where human beings offer the most value to the system. As past experience and expert knowledge levels become players, the machine's role becomes the tool for human intervention. The decision-making prowess of the individual is a critical component in the human-machine unit, and so it is imperative to embrace human problem solving dexterity in conjunction with the commanding process.

7.2.6.5 Summary. As previously mentioned, these are merely suggestions for redesign that could be incorporated into a follow-on study to this project. This future study would focus on optimization of the "machine" portion of the human-machine system. Variation of machine technology could provide useful data on desired system characteristics assuming the operator knowledge level was held constant. After determining the desired operator knowledge level, the overall system could then be more effectively designed for the optimal operator. 


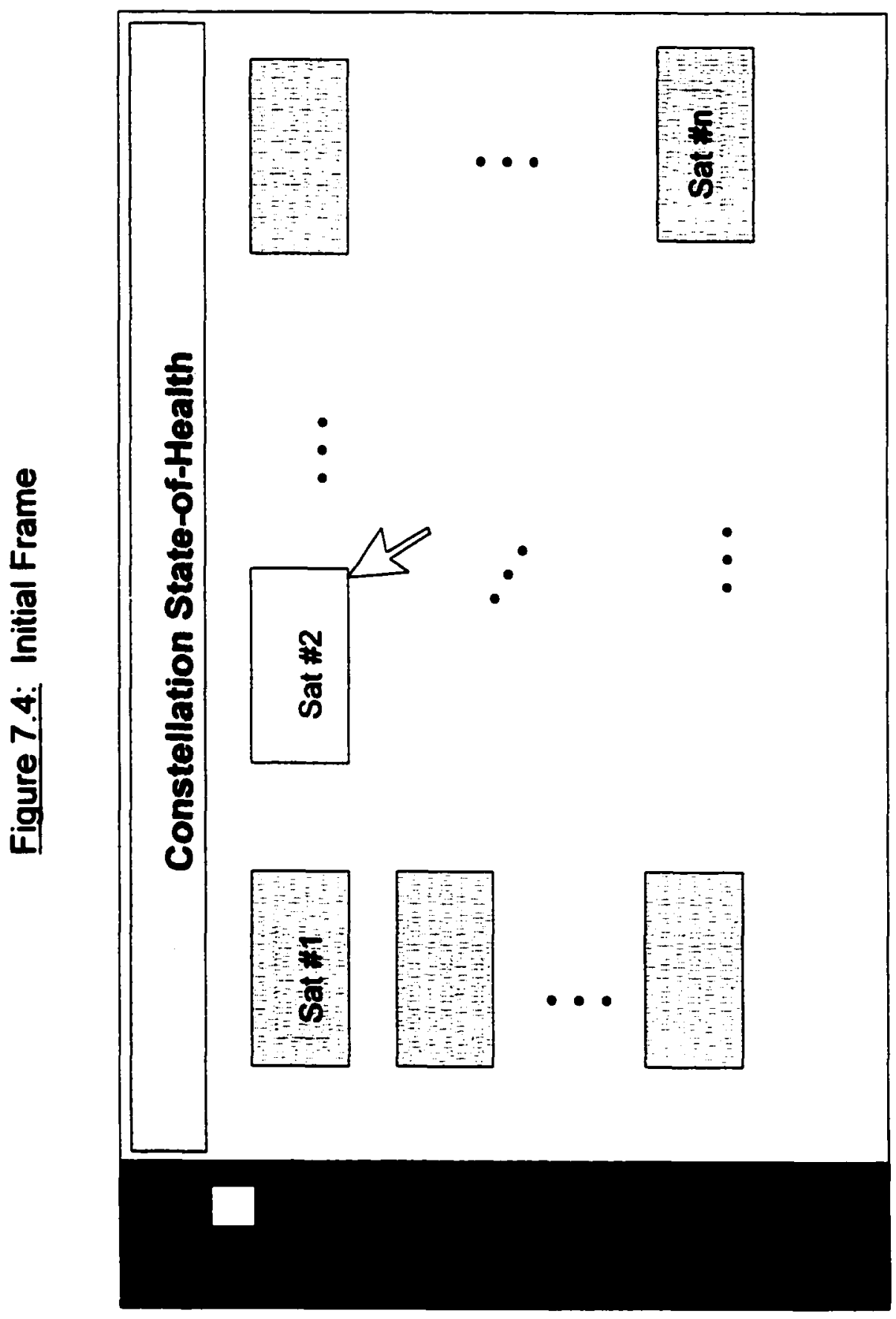

$\stackrel{\infty}{\leftarrow}$ 
Figure 7.5: Subsystem Level

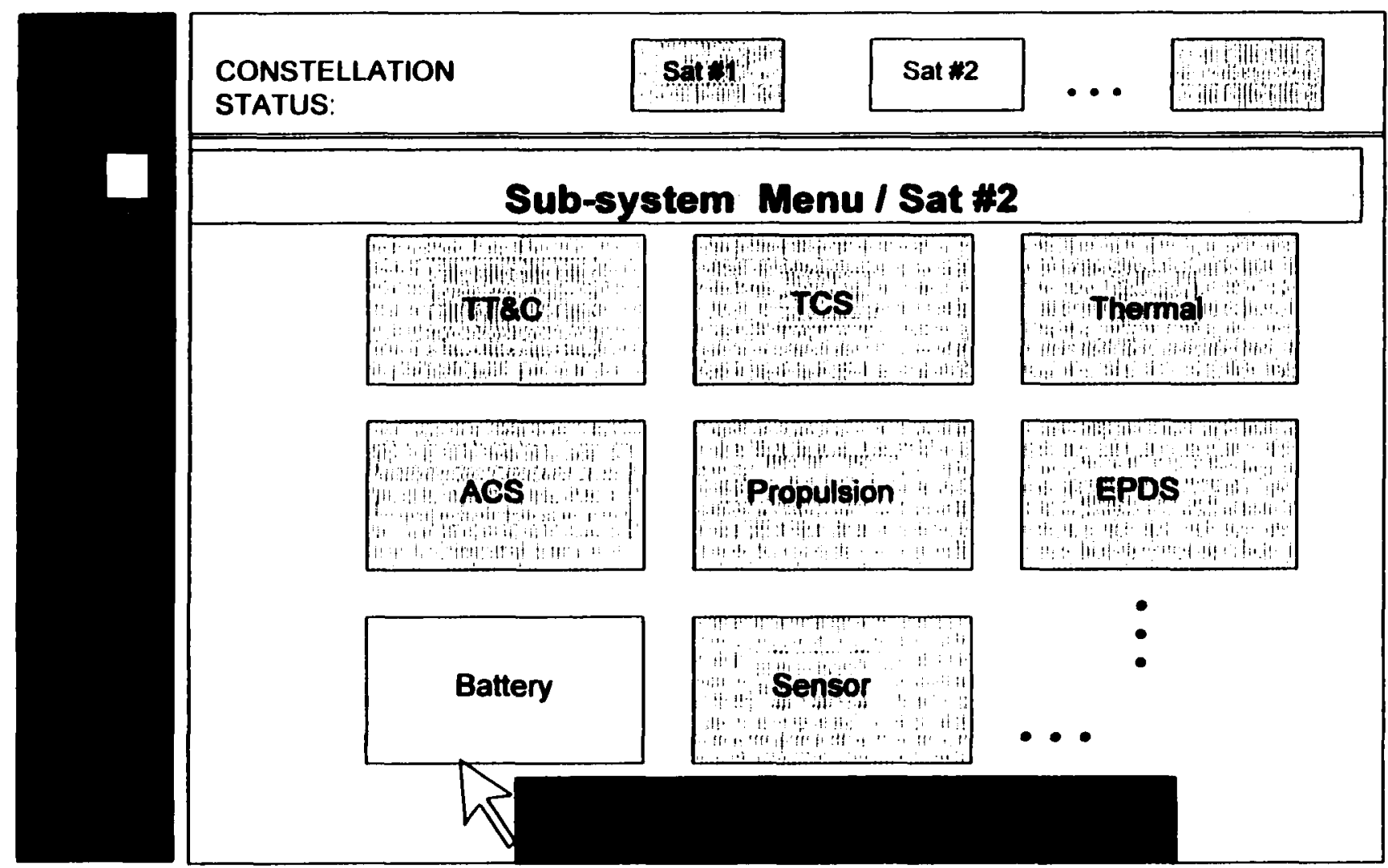


Figure 7.6: Subsystem Level - Detail

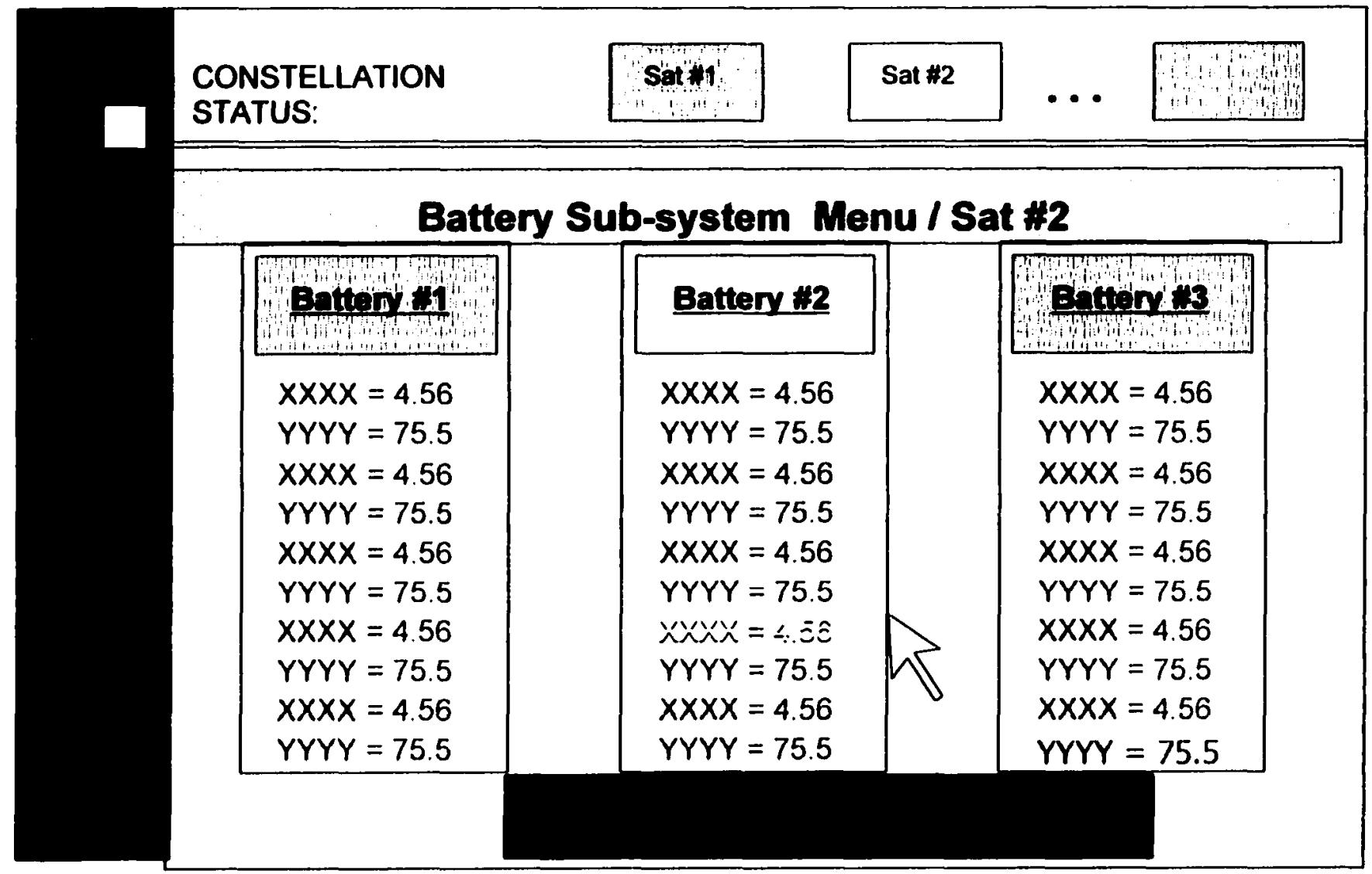


Figure 7.7: Mnemonic Explanation

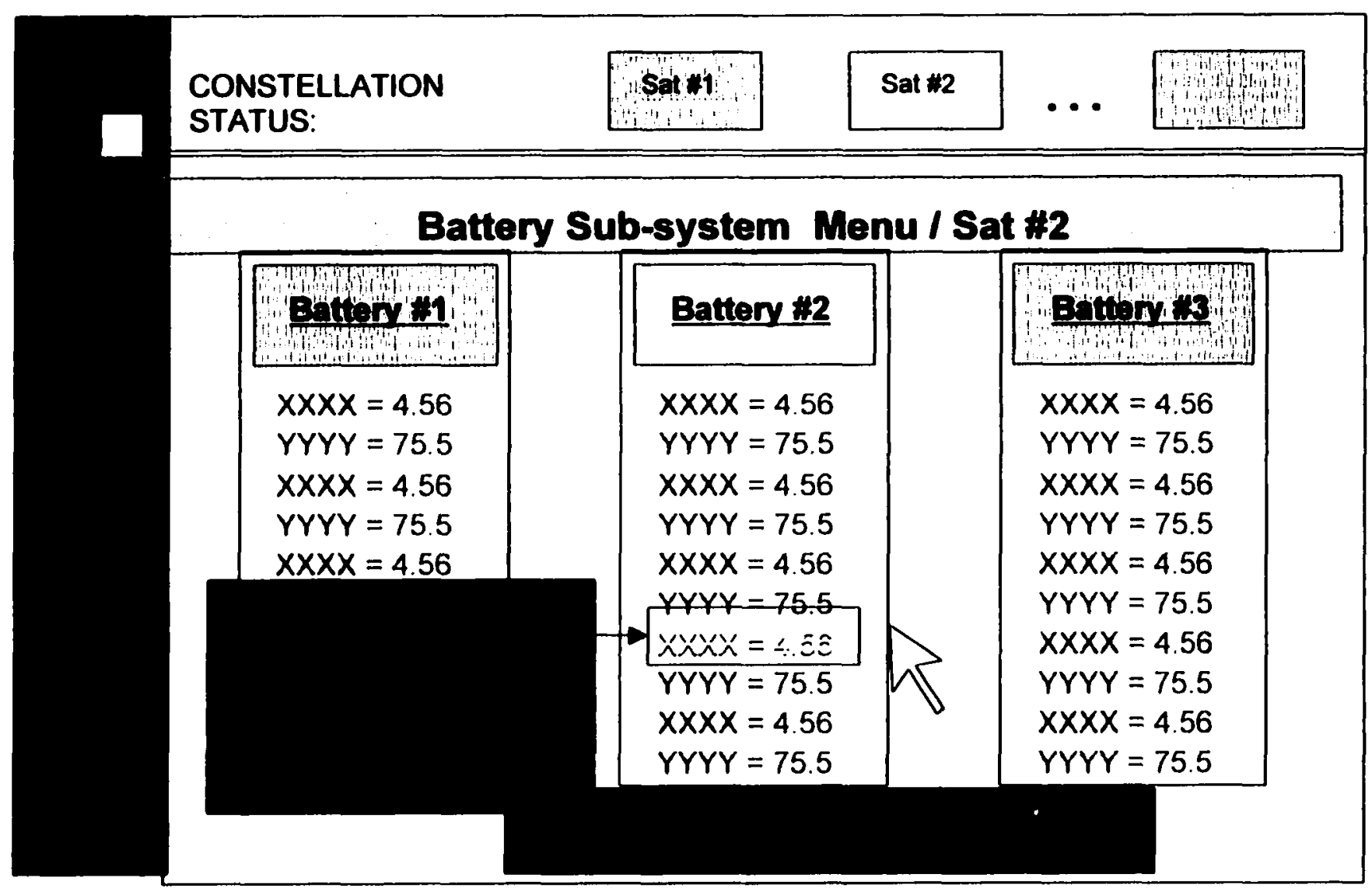


Figure 7.8: Commanding Window

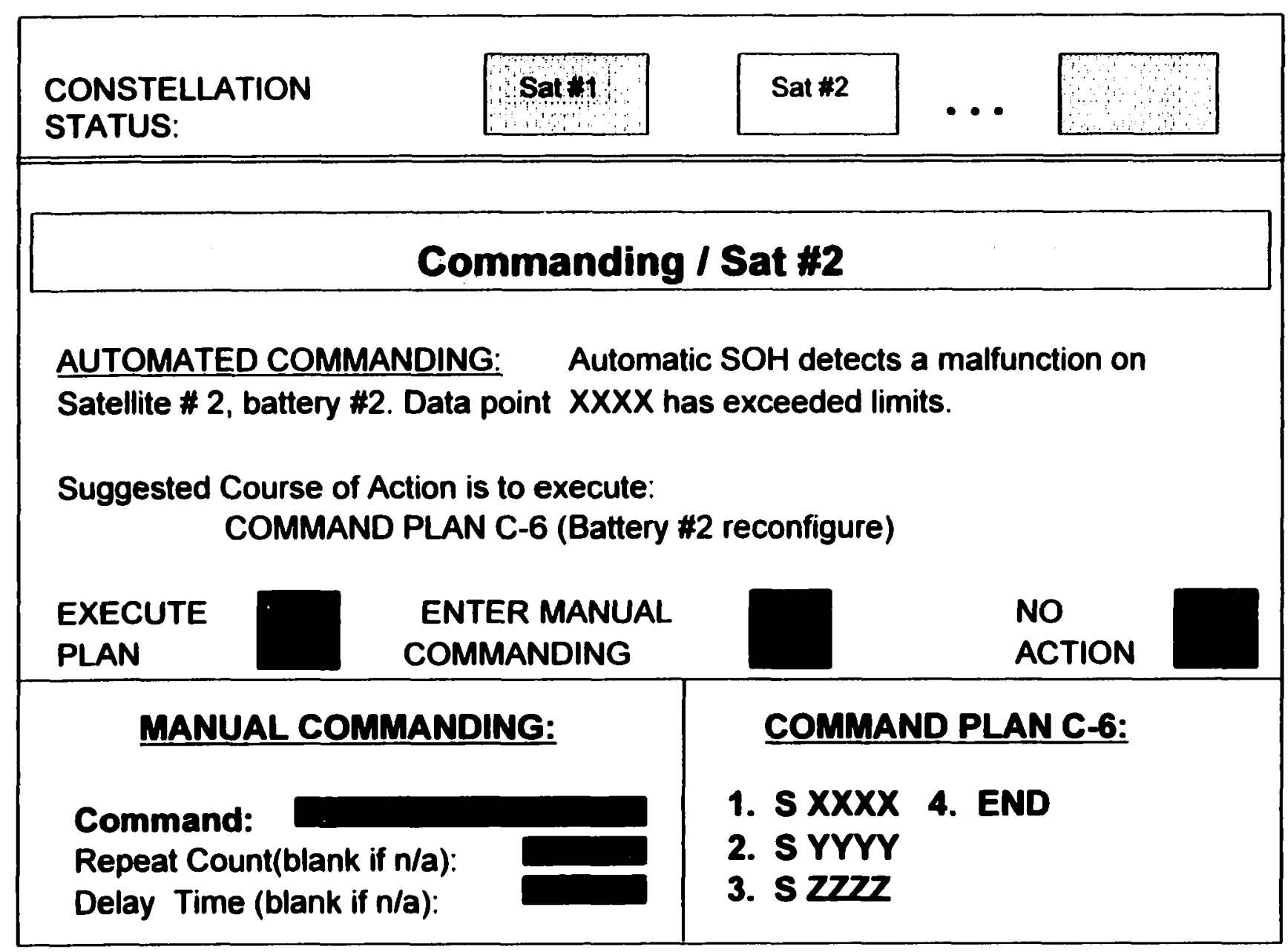


Estimations of expert operator improvement for recognizing and identifying anomalies are identified below. These are purely hypothesized improvement statistics.

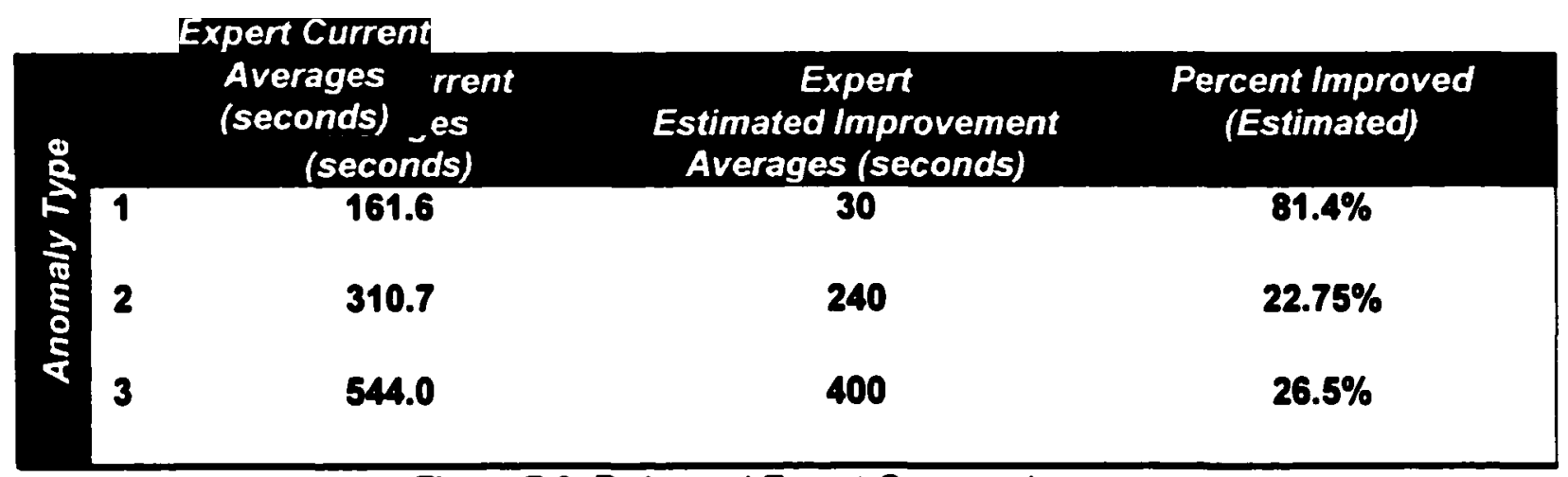

Figure 7.9: Estimated Expert Operator Improvement

Specific to the outcome of this study, it is expected that screen redesign would have a significant effect on the expert operator. It would allow them to be free of vegetative, caretaker-tasks and allow them to use their expertise where it is desperately needed: experts would be able to perform more non-deterministic, decision making tasks. More significantly, they could focus efforts on auxiliary tasks of mission planning, orbit analysis and other responsibilities, possibly eliminating the need for additional personnel to perform those necessary functions. It is important to note that any redesign should be accompanied by extensive testing for functionality and operator usability.

7.2.7 Follow-on studies. Follow-on studies are necessary. Satellite command and control optimization consists of not only the optimal operator, but also an optimal machine-system. This research represents only one of three possible examinations of this perplexing issue:

1) Focus on operator knowledge by varying user experience and training and maintaining machine technology as a constant (this study) 
2) Focus on machine capability by varying technology and maintaining operator knowledge as a constant (future study)

3) Optimize the entire human-machine system further by employing a human centered design strategy during the optimization process (future study).

\subsection{Significance and Implications}

The Defense Support Program satellites cost roughly $\$ 375$ million per vehicle. The exceptional cost and criticality associated with this system (and other similar complex systems) argue the necessity of precise and timely system control. As it stands, anomaly resolution is a time-consuming and cumbersome process. The claim is that by placing the decision-making and knowledge base into the system through the human, in combination with an interface that facilitates troubleshooting, adds automation, and informatively presents data by filtering out unnecessary distracters, we can significantly improve timeliness and accuracy of SOH'S and planned/emergency commanding. This goal can be accomplished in conjunction with freeing up precious Remote Tracking Station resources and other Air Force Space Command assets by decreasing the amount of antenna time required for operations (current baseline is 10 minutes for nominal ranging and $\mathrm{SOH}$ missions). This study represents "Part 1" of an optimization study; "Part 2" should focus solely on the machine side. "Part 3" could continue the optimization of both user and machine to create a human-machine system capable of adapting to the rapidly changing space environment and able to contribute more fully to space control objectives. 


\section{LIST OF REFERENCES}




\section{LIST OF REFERENCES}

$\left[1^{\text {st }}\right.$ Space Operations Sqdn, 2000] SOPS/DOUP, 1 SOPS Operator Checklists, $1^{\text {st }}$ Space Operations Squadron, 50 SW, AFSPC. Schriever AFB, CO, Oct 2000.

[1 ${ }^{\text {st }}$ Space Operations Sqdn, 2000] 1 SOPS/DOUP, 1 SOPS Operations Handbook (DSP/GPS/TAOS/MSX/ACE), $1^{\text {st }}$ Space Operations Squadron, 50 SW, AFSPC, Schriever AFB, CO, 2000.

[1 ${ }^{\text {st }}$ Space Operations Sqdn, 2000]

[Aerojet Corporation, 1996]

[Acton, et al., 1986]

[Battiste, et al., 1988]

[Billings, 1997]

[Burns, 2000]

[Chappell, et al., 1995]
1 SOPS/DOUE, 1 SOPS DSP Vehicle Binders, $1^{\text {st }}$ Space Operations Squadron, 50 SW, AFSPC, Schriever AFB, CO, 2000.

GenCorp/AeroJet Corp., Contingency Operations Handbook for DSP-I Sensors, Contract F04701-92-C. 0026, Los Angeles AFB, CA, Change 2, Feb 1996.

Acton, W., W. Perez, G. Reid, On the dimensionality of subjective workload, Proceedings of the Human Factors Society 30th Annual Meeting, Vol. 1. pp. 76-80, 1986.

Battiste, V., M. Bortulussi, Transport pilot workload: a comparison of two subjective measure techniques, Proceedings of the Human Factors Society 32nd Annual Meeting, Anaheim, CA, Vol. 1, pp. 150-154, 1988.

Billings, C., Aviation Automation: The Search for a Human Centered Approach, Lawrence Erlbaum Assoc. Publishers, New Jersey, 1997.

Burns, C. Putting it all together: improving display integration in ecological displays, Human Factors, Vol. 42, No. 2, pp. 226-241, 2000.

Chappell, A., C. Mitchell, Addressing the trained novice/expert performance gap in complex dynamic systems: a case based intelligent tutoring system. Proceedings of the IEEE Conference on Syst., Man and Cyber., Vancouver, BC, Can, Vol. 5, pp. 4557. 4562, 1995. 
[Chavez-Mercado, et al., 1995]

[Chu, et al., 1991]

[Chu, et al., 1995]

[Cochran, 1963]

[Cox, et al. 1984]

[Davies, 1956]

[Driskell, et al., 1989]

[Elandt-Johnson, et al. 1999]

[Elvers, et al., 1993]

[Endsley, et al., 1995]
Chavez-Mercado, C., B. Zayas-Perez, J. ValdezParra, H. Ocampo-Mansilla, The classroom analysis simulator: a training and research tool for nuclear power plant operations, Proceedings of the IEEE Conference on Human Factors and Power Plants, Orlando, FL, pp. 17.11-17.16, 1997.

Chu, R., C. Mitchell, GT-VITA: an intelligent tutoring and aiding system for satellite ground control, , Proceedings of the IEEE Conference on Syst., Man and Cyber., Vol. 3, pp. 1745-1750, 1991.

Chu, R., C. Mitchell, P. Jones, Using the operator function model and OFMspert as the basis for an intelligent tutoring system: towards a tutor/aid paradigm for operators of supervisory control systems, IEEE Transactions on Systems, Man and Cybernetics, Vol. 25, No. 7, pp. 1054-1075, 1995.

Cochran, W., Sampling Techniques,

Wiley and Sons, New York, 1963.

Cox, D., D. Oakes, Analysis of Survival Data,

Chapman and Hall, New York, 1984.

Davies, 0 . The Design and Analysis of Industrial Experiments, $2^{\text {nd }} e d$., Oliver and Boyd, Edinburgh, 1956.

Driskell, J., B. Olmstead. Psychology and the Military, American Psychologist, Vol. 44, No. 1, pp. 4354, 1989.

Elandt-Johnson, R., N. Johnson, Survival Models and Data Analysis, John Wiley and Sons, New York, 1999.

Elvers, G., R. Adapathya, K. Klauer, D. Kancler, N. Dolan, Effects of task probability on integral and separable task performance, Human Factors, Vol. 35 , No. 4, pp. 629-637, 1993.

Endsley, M., E. Kiris, The out of the loop performance problem and level of control in automation, Human Factors, Vol. 37, No. 2, pp. 381394, 1995. 
[Endsley, et al., 1999]

[Furuta, et al., 1999]

[Gibson, et al., 1988]

[Graine, 1988]

[Hammer, et al., 1988]

[Hammer, et al., 1996]

[Hancock, et al., 1989]

[Hendrick, et al., 1992]

[Hendy, et al., 1993]
Endsley, M., D. Kaber, Level of automation effects on performance, situation awareness, and workload in a dynamic control task, Ergonomics, Vol. 42, No. 3, pp. 462-492, 1999.

Furuta, K., K. Furuhama, Cognitive space of operator's knowledge, Ergonomics, Vol 42, No. 11, pp. 1431-1442, 1999.

Gibson, D., G. Salvendy, Knowledge representation in human problem solving: implications for expert system design, Proceedings of the Human Factors Society 32nd Annual Meeting, Anaheim, CA, Vol. 1, pp. 395-398, 1988.

Graine, G., The Engineering Syndrome Versus the Manpower Personnel and Training Dilemma, Naval Engineers Journal, pp. 54-58, 1988.

Hammer, A., G. Birdwell, H, Snyder, Optimization of user and system knowledge. Proceedings of the Human Factors Society 32nd Annual Meeting, Anaheim, CA, Vol. 1, pp. 399-403, 1988.

Hammer, J., R. Small. An Intelligent Interface in and Associate System, Human/Technology Interaction in Complex Systems, JAI Press, Greenwich, CT, Vol. 7, 1996.

Hancock, P., M. Robinson, A. Chu, D. Hansen, M. Vercruyssen, E. Grose, A. Fisk, The effects of practice on tracking and subjective workload, Proceedings of the Human Factors Society 33rd Annual Meeting, Vol. 1, pp. 1310-1313, 1989.

Hendrick, H., M. Grabowski, A process for developing functional generic models for determining personnel requirements, Proceedings of the Human Factors Society 36th Annual Meeting, Vol. 1, pp. 838842, 1992.

Hendy, K., K. Hamilton, L. Landry, Measuring subjective workload: when is one scale better than many?, Human Factors, Vol. 35, No. 4, pp. 579-601, 1993. 
[Hill, et al., 1998]

[Hoffman, et al., 1998]

[Hogg, et al., 1983]

[Huey, et al., 1992]

[Hull, 1943]

[Johnson, 1997]

[Jones, et al., 1989]

[Jones, et al., 1990]

[Jones, et al., 1995]

[Jones, et al., 1995]
Hill, R., S. Chien, K. Fayyad, Goal-driven automation of a deep space communications station: a case study in knowledge engineering or plan generation and execution, Expert Systems, Vol. 15, No. 3, pp. 141-156, 1998.

Hoffman, R., B. Crandall, N. Shadbolt, Use of critical decision method to elicit expert knowledge: a case study in the methodology of cognitive task analysis, Human Factors, Vol. 40, No. 2, pp. 254-276, 1998.

Hogg, R., E. Tanis, Probability and Statistical Inference, Macmillan Publishing, New York, 1983.

Huey, B., D. Boehm-Davis, Individual differences in the performance of a process control task, Proceedings of the Human Factors Society 36th Annual Meeting, Vol. 1, pp. 838-842, 1992.

Hull, C., Principles of Behavior, Appleton-CenturyCrofts, New York, 1943.

Johnson, D., Getting the message across: conveying understandable process information to an operator requires more than dazzling HMl graphics in real time, Control Engineering, Vol. 44, No. 12. (Internet Article, 6 pp.), 1997.

Jones, P., C. Mitchell, Operator models for supervisory control systems, Proceedings of the Human Factors Society 33rd Annual Meeting, Vol. 1, pp. 291-295, 1989.

Jones, P., C. Mitchell, K. Rubin, Validation of intent inferencing by a model-based operator's associate, International Journal of Man-Machine Studies, Vol 33, pp. 177-202, 1990.

Jones, P., R. Chu, C. Mitchell, A methodology for human-machine systems research: knowledge engineering, modeling, and simulation, IEEE Trans. Syst., Man and Cyber., Vol 25, pp. 1035-1038, 1995.

Jones, P., C. Mitchell, Human-computer cooperative problem solving: theory, design, and evaluation of an intelligent associate, IEEE Trans. Syst., Man and Cyber., Vol 25, pp. 1039-1053, 1995. 
[Kaempf, et al., 1996]

[Kantowitz, et al., 1990]

[Kerstholt, et al., 1996]

[Kilmer, et al., 1988]

[Kuehl, 2000]

[Lee, 1992]

[Lentner, et al., 1993]

[Luximon, et al., 2001]

[Montgomery, 1991]

[Moroney, et al., 1995]

[Namboodiri, et al., 1975]
Kaempf, G., G. Klein, M. Thorsden, S. Wolf, Decision making In complex naval command and control environments, Human Factors, Vol. 38, No. 2 , pp. 220-231, 1996.

Kantowitz, B., A. Bittner, Y. Fujita, Mathematical description of crew response times in simulated nuclear power plant emergencies, Proceedings of the Human Factors Society $34^{\text {th }}$ Annual Meeting, Orlando, FL, Vol. 2, pp. 1127-1132, 1990.

Kerstholt, J., P. Passenier, K. Houttuin, H. Schuffel, The effect of a priori probability and complexity on decision Making in a Supervisory Control Task, Human Factors, Vol. 38, No. 1, pp. 65-78, 1996.

Kilmer, K., R. Knapp, C. Burdsall, R. Borresen, R. Bateman, D. Malzahn, Techniques of subjective assessment: a comparison of the SWAT and Modified Cooper-Harper Scales, , Proceedings of the Human Factors Society 32nd Annual Meeting, Anaheim, CA, Vol. 1, pp. 155-158, 1988.

Kuehl, R., Design of Experiments: Statistical Principles of Research Design and Analysis, Duxbury Press, Belmont, California, 2000.

Lee, E., Statistical Methods for Survival Data Analysis, John Wiley and Sons, New York, 1992.

Lentner, M., T. Bishop, Experimental Design and Analysis, Valley Book Co., Blacksburg, Virginia, 1993.

Luximon, A., R. Goonetilleke, Simplified subjective workload assessment technique, Ergonomics, Vol 44, No. 3, pp. 229-243, 2001.

Montgomery, D., Design and Analysis of Experiments, Wiley and Sons, New York, 1991.

Moroney, W., A. Bittner, Military Systems Techniques, (Article in Research Techniques in Human Engineering), Prentice Hall, New Jersey, 1995.

Namboodiri, N., L. Carter, H. Blalock. Applied Multivariate Analysis and Experimental Designs, McGraw Hill, New York, 1975. 
[Northern NEF, 2000]

[Ostle, et al., 1988]

[Parasuraman, et al., 1996]

[Reid, et al., 1988]

[Reid, et al., 1988]

[Reid, et al., 1989]

[Roth, et al., 1986]

[Rohles, 1988]

[Rueb, et al., 1991]

[Rueb, et al., 1992]
Northern NEF, Inc., Rehearsal Enqineer's Manual: Technical Report, Space Operations Training Support, Colorado Springs, CO, Contract FA2550-97-C-0003, Sep 2000, 35 pp.

Ostle, B., L. Malone, Statistics in Research, lowa State University Press, Ames, lowa, 1988.

Parasuraman, R., M. Mouloua, R. Molloy, Effects of adaptive task allocation of monitoring of automated systems, Human Factors, Vol. 38, No. 4, pp. 665-679, 1996

Reid, G., T. Nygren, Subjective Workload Assessment Technique (SWAT): a scaling procedure for measuring mental workload, Conference on Human Mental Workload, Amsterdam, The Netherlands: North Holland, 1988.

Reid, G., H. Colle, Critical SWAT values for predicting operator overload. Proceedings of the Human Factors Society, $32^{\text {nd }}$ Annual Meeting, pp. 1414-1418, 1988.

Reid, G., S. Potter, J. Bressler, Subjective Workload Assessment Technique (SWAT): a User's Guide, Interim Report, Armstrong Aerospace Medical Research Laboratory, Human Systems Division, WPAFB, OH, 115 pp., July 1989.

Roth, E., D. Woods, Analysis of expertise in a dynamic control task, Proceedings of the Human Factors Society 30th Annual Meeting, Vol. 1, pp. 179181, 1986.

Rohles, F., A model for describing performance and productivity, Proceedings of the Human Factors Society 32nd Annual Meeting, Anaheim, CA Vol. 1, pp. 519-521, 1988.

Rueb, J., M. Vidulich, J. Hassoun, Use of workload redilines: a KC-135 crew-reduction application, International Journal of Aviation Psychology, Vol. 4, No. 1, pp. 47-64, 1991.

Rueb, J., M. Vidulich, J. Hassoun, Establishing workload acceptability: an evaluation of a proposed KC-135 cockpit redesign. Proceedings of the Human Factors Society $36^{\text {th }}$ Annual Meeting. Vol. 1, pp. 17-21, 1992. 
[Rubin, et al., 1988]

[Sall, et al., 1996]

[Sarter, et al., 1997]

[Scheffe, 1959]

[Selvin, 1991]

[Selvin, 1995]

[Sirken, et al., 1999]

[Stanislaw, 1995]

[Sutton, 1990]

[Sverdup Technology, 2000]

[Switzer, et al., 1989]
Rubin, K., P. Jones, C. Mitchell, OFMspert: Inference of operator intentions in supervisory control using a blackboard architecture, IEEE Transactions on Systems, Man, and Cybernetics, Vol. 18, No. 4, pp. 618-636, 1988.

Sall, J., A. Lehman, JMP Start Statistics: A Guide to Statistics and Data Analysis Using JMP and JMP IN Software, Duxbury Press, Belmont, California, 1996.

Sarter, N., D. Woods, Team play with a powerful and independent agent: operational experience and automation surprises on the airbus A-320. Human Factors, Vol. 39, No. 4, pp. 553-568, 1997.

Scheffe, H., The Analysis of Variance, Wiley and Sons, New York, 1959.

Selvin, S., Statistical Analysis of Epidemiologic Data, Oxford Univ. Press, New York, 1991.

Selvin, S., Practical Biostatistical Methods, Duxbury Press, Belmont, California, 1995.

Sirken, M., D. Hermann, S. Schechter, N. Schwarz, $J$ J Tanur, R. Tourangeau, Cognition and Survey Research, Wiley and Sons, New York, 1999.

Stanislaw, $H_{\text {., Inspector performance of an }}$ industrial inspection-type task, Human Factors, Vol. 37. No. 1, pp. 182-192, 1995.

Sutton, R., Modeling Human Operators in Control System Design, Research Studies Press, LTD., Taunton, England, 1990.

Sverdup Technology, Software Requirements Specification for the Air Force, $1^{\text {st }}, 3^{\text {td }}$, and $4^{\text {th }}$ Space Operations Squadrons Command and Control System Crew Trainer Emulator (CCS-CTE), Clearfield, UT, Contract No. F42620-00-D0036-0004, 71pp., 2000.

Switzer, F., J. Idaszak, Effects of crew coordination and level of instruction on process control operator, Proceedings of the Human Factors Society 33rd Annual Meeting, Vol. 1, pp. 788-791, 1989. 
[TRW Corporation, 1987]

[TRW Corporation, 1988]

[TRW Corporation, 1994]

[TRW Corporation, 1994]

[TRW Corporation, 1994]

[TRW Corporation, 1994]

[TRW Corporation, 1994]

[TRW Corporation, 1997]

[TRW Corporation, 1997]

[TRW Corporation, 1997]
TRW Space and Electronics Group, DSP Orbital Operations Handbook (OOH), Satellites 0005R/6R, Vols. 1-14, Revision 004, 15 Sep 1987.

TRW Space and Electronics Group, Satellites 5R/6R and 14/17 Differences Document, Redondo Beach, CA, Contract F05603-87-C-0021, Dec 1988.

TRW Space and Electronics Group, DSP-1 Technical Documentation, Subsystem Manual, Volume 1 (Spacecraft Overview), Redondo Beach, CA, Contract F04701-91-C-0002, Dec 1994.

TRW Space and Electronics Group. DSP-1 Technical Documentation. Subsystem Manual, Volume 3 (Thermal Control), Redondo Beach, CA, Contract F04701-91-C-0002, Dec 1994.

TRW Space and Electronics Group, DSP-1 Technical Documentation, Subsystem Manual, Volume 4 (Communications and Command), Redondo Beach, CA, Contract F04701-91-C-0002, Dec 1994.

TRW Space and Electronics Group, DSP-1 Technical Documentation, Subsystem Manual. Volume 6 (Electrical Power and Distribution Subsystem), Redondo Beach, CA, Contract F04701-91-C-0002, Dec 1994.

TRW Space and Electronics Group, DSP-1 Technical Documentation. Subsystem Manual, Volume 8 (Attitude Control), Redondo Beach, CA, Contract F04701-91-C-0030, Dec 1994.

TRW Space and Electronics Group DSP-1 Technical Documentation. Subsystem Manual, Volume 5 (Mission Data Message), Redondo Beach, CA, Contract F04701-96-C-0030, Feb 1997.

TRW Space and Electronics Group, DSP-1 Technical Documentation. Subsystem Manual. Volume 2 (Spacecraft Structure), Redondo Beach, CA, Contract F04701-97-C-0030, Feb 1997.

TRW Space and Electronics Group, DSP-1 Technical Documentation, Subsystem Manual, Volume 7 (Propulsion), Redondo Beach, CA, Contract F0470196-C-0030, Feb 1997. 
[TRW Corporation, 1997]

[Turner, et al., 1991]

[Turner, 1996]

[Urban, et al., 1992]

[Vasandami, et al., 1995]

[Vidulich, et al., 1991]

[Wackerly, et al., 1996]

[Weimer, 1995]

[Wells, et al., 1989]

[Wheeler, et al., 1990]
TRW Space and Electronics Group, DSP-1

Technical Documentation, Subsystem Manual, Volume 8 (Attitude Control), Redondo Beach, CA, Contract F04701-96-C-0030, Feb 1997.

Turner, S., P. Mangan, M. Meloan, C. Simmons, Prototype expert system workstation for satellite anomaly resolution, Journal of Spacecraft and Rockets, Vol. 28, No.1, pp. 352-354, 1991.

Turner, A., A Paired Comparison Method for Interval Scaling, Human Factors, Vol. 38, No. 2, pp. 362-374, 1996.

Urban, J., C. Bowers, B. Morgan, C. Braun, P. Kline, The effects of hierarchical structure and workload on the performance of team and individual tasks, Proceedings of the Human Factors Society $36^{\text {th }}$ Annual Meeting, Vol. 1, pp. 829-833, 1992.

Vasandami, V., T. Govindaraj, Knowledge organization in intelligent tutoring systems for diagnostic problem solving in complex dynamic domains, IEEE Trans. Syst., Man and Cyber., Vol 25. pp. 1076-1096, 1995.

Vidulich, M., G. Ward, J., Schueren, Using the subjective workload dominance (SWORD) technique for projective workload assessment, Human Factors, pp. 678-691, 1991.

Wackerly, D., W. Mendenhall, R. Scheaffer, Mathematical Statistics with Applications, Duxbury Press, Belmont, California, 1996.

Weimer, J., Research Techniques in Human Engineering, Prentice Hall, New Jersey, 1995.

Wells, M., M. Venturino, The effect of task complexity on the field-of-view requirements for a visually coupled system, Proceedings of the Human Factors Society 33rd Annual Meeting, Vol. 1, pp. 9195, 1989.

Wheeler, W., P. Bolton, T. Sanquist, Decision making in an emergency: when information is not enough, Proceedings of the Human Factors Society $34^{\text {th }}$ Annual Meeting, Orlando, FL, Vol. 2, pp. 1137 1141, 1990. 
Wickens, et al., 1989]

[Williges, 1995]

[Woods, et al., 1990]
Wickens, C., R. Marsh, M. Raby, S. Straus, R.

Cooper, C. Hulin, F. Switzer, Aircrew performance as a function of automation and crew composition: a simulator study, Proceedings of the Human Factors Society 33rd Annual Meeting. Vol. 1, pp. 792-796, 1989.

WilligesR., Review of Experimental Design (Article in Research Techniques in Human Engineering), Prentice Hall, New Jersey, 1995.

Woods, D., E. Roth, H. Pople, Modeling operator performance in emergencies, Proceedings of the Human Factors Society $34^{\text {th }}$ Annual Meeting, Orlando, FL, Vol. 2, pp. 1132-1135, 1990. 


\section{APPENDICES}

Reproduced with permission of the copyright owner. Further reproduction prohibited without permission. 


\section{Appendix A}

\section{RAW DATA AND STATISTICAL ANALYSIS}

This appendix includes raw data and a more detailed explanation of the methods used to analyze the scenario results. 


\begin{tabular}{|c|c|}
\hline Scenario & Description \\
\hline 1 & Impact sensor/OOL battery points \\
\hline 1 & Solar array damage \\
\hline 1 & Command bals, power conservation \\
\hline 2 & Low plenum pressure \\
\hline 2 & Main tank propellant leak \\
\hline 2 & Find alternate propellant paln \\
\hline 3 & EMI \\
\hline 3 & Broken transmitter/switch to $2 B$ \\
\hline 3 & Broken receiver/switch to $R \times 3 B$ \\
\hline 4 & Star Sensor Closed \\
\hline 4 & Command power offfino functional \\
\hline 4 & Switch to SSPA.B \\
\hline 5 & Unexpected command counts \\
\hline 5 & Venicle in eclipse \\
\hline 5 & Command batteries and monitor voltage \\
\hline 6 & Incorrect TCS mode/OOL nellum points \\
\hline 6 & TesUconfirm helium leak \\
\hline 6 & Swap to alternate TCS \\
\hline 7 & Pump plenum on B side \\
\hline 7 & Plenum leak \\
\hline 7 & Swap Control Electronics Assy-A to $B$ \\
\hline
\end{tabular}

\begin{tabular}{|c|c|c|c|c|}
\hline Assumed Type & Time/Standard 1 & Timastundard 2 & TimerExpen 1 & Time/Expert 2 \\
\hline 1 & 202 & 693 & 447 & 279 \\
\hline 2 & 589 & 148 & 449 & 859 \\
\hline 3 & $2807 \cdot \cdot$ & 955 & 453 & 335 \\
\hline 1 & 295 & 1103 & 119 & 93 \\
\hline 2 & 11 & 192 & 220 & 138 \\
\hline 3 & 2666 & 2166 & 1655 & 806 \\
\hline 1 & 66 & 323 & $3600 * *$ & 70 \\
\hline 2 & 958 & 968 & 694 & 234 \\
\hline 3 & 318 & 1633 & 1007 & 69 \\
\hline 1 & 82 & 158 & 82 & 326 \\
\hline 2 & 397 & 416 & 157 & 33 \\
\hline 3 & 154 & 85 & 73 & 60 \\
\hline 1 & 217 & 518 & 273 & 238 \\
\hline 2 & 55 & 274 & 109 & 66 \\
\hline 3 & 182 & 265 & 436 & 354 \\
\hline 1 & 89 & 1303 & 85 & 149 \\
\hline 2 & $3510^{\circ}$ & $2296 * \bullet$ & 115 & 169 \\
\hline $\mathbf{3}$ & 515 & 35 & 173 & 167 \\
\hline 1 & 202 & 99 & 173 & 330 \\
\hline 2 & 251 & 357 & 609 & 129 \\
\hline 3 & 1493 & 1615 & 1924 & 647 \\
\hline
\end{tabular}

Figure A.1: Raw Time Data ("* Indicates censored values) 


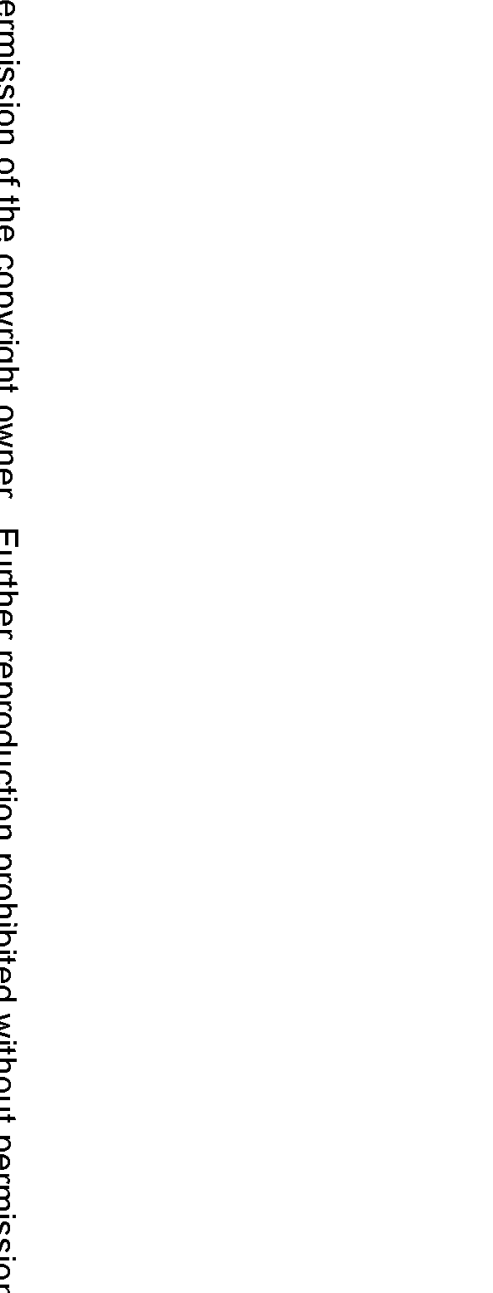

\begin{tabular}{|c|c|c|c|c|}
\hline Assumed Type & Timesstd 1 & Timerstd 2 & TimedExpent 1 & TimedExpert \\
\hline 1 & 58 & 78 & 145 & 39 \\
\hline 2 & 1138 & 348 & 676 & 347 \\
\hline 3 & 389 & 604 & 568 & 212 \\
\hline 1 & 619 & 410 & 332 & 262 \\
\hline 2 & 218 & 69 & 188 & 98 \\
\hline 3 & 251 & 1141 & 357 & 334 \\
\hline 1 & 227 & 206 & 125 & 88 \\
\hline 2 & 1140 & 310 & 1005 & 405 \\
\hline 3 & 1486 & 640 & 175 & 392 \\
\hline 1 & 91 & 224 & 170 & 147 \\
\hline 2 & 87 & 183 & 87 & 42 \\
\hline 3 & 270 & 261 & 77 & 100 \\
\hline 1 & 115 & 348 & 303 & 95 \\
\hline 2 & 241 & 396 & 67 & 47 \\
\hline 3 & $3243^{\circ}$ & $2854^{\circ *}$ & 2364 & 278 \\
\hline 1 & 50 & 140 & 75 & 42 \\
\hline 2 & 396 & 618 & 545 & 300 \\
\hline 3 & 339 & 800 & 696 & 163 \\
\hline 1 & 85 & 90 & 60 & 80 \\
\hline 2 & 516 & 353 & 1023 & 208 \\
\hline 3 & 576 & 805 & 1355 & 813 \\
\hline 1 & 126 & 197 & 90 & 60 \\
\hline 2 & 77 & 505 & 138 & 163 \\
\hline 3 & 554 & 362 & 169 & 107 \\
\hline
\end{tabular}

Figure A.1: Raw Time Data (Continued) 
Figure A.2: SWAT Ratings

Leqend:

difficulty $=$ Overall perceived anomaly difficulty [scale $1-10,10$ being most difficult]

NOTE: Scale is 1,2 , or 3 for T. E, and S SWAT ratings

$T=$ Perceived time load

$E=$ Perceived mental effort load

$\mathbf{S}=$ Perceived psychological stress load

\begin{tabular}{|c|c|c|c|c|c|c|c|c|c|c|}
\hline SCENARIO & ANOMALY & LOAD & \multicolumn{3}{|c|}{ STO CREW 1} & \multicolumn{3}{|c|}{ STO CREW 2} & EXPERT 1 & EXPERT 2 \\
\hline & & & ENG & Svo- & SV0-2 & ENG & SVo & SV0-2 & OP & OP \\
\hline : & 1 & difticinitivi & : & : & (1) & : & 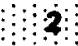 & 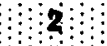 & :3: & 男 \\
\hline 1 & 2 & difficuliti & 3 & : & 2 & 7 & 3 & 0 & 6 & $10:$ \\
\hline 1 & 3 & difficulity & ia & o & 10 & 10 & : & 9 & 9 & 10 \\
\hline 1 & $i$ & $\mathbf{T}$ & 1 & 1 & 1 & 2 & 1 & 2 & 2 & 1 \\
\hline 1 & 1 & E & 1 & 1 & 1 & 2 & 2 & 2 & 1 & 1 \\
\hline 1 & 1 & $\mathbf{s}$ & 1 & 1 & 1 & 2 & 1 & 2 & 1 & 1 \\
\hline 1 & 2 & $\mathbf{T}$ & 1 & 2 & 1 & 1 & 1 & 1 & 2 & 3 \\
\hline 1 & 2 & $\mathbf{E}$ & 2 & 1 & 2 & 2 & 1 & 2 & 2 & 3 \\
\hline 1 & 2 & $\mathbf{s}$ & 2 & 1 & 1 & 2 & 1 & 2 & 3 & 3 \\
\hline 1 & 3 & $\mathbf{T}$ & 3 & 2 & 1 & 2 & 2 & 3 & 2 & 3 \\
\hline 1 & 3 & $E$ & 3 & 3 & 3 & 3 & 3 & 3 & 3 & 3 \\
\hline 1 & 3 & s & 3 & 3 & 3 & 3 & 2 & 2 & 3 & 3 \\
\hline 2 & 1 & difficulty & 2 & 1 & 1 & 2 & 2 & 2 & 2 & 1 \\
\hline 2 & 2 & dificiolity & 3 & 2 & 3 & 6 & $\mathbf{3}$ & 4 & 2 & 3 \\
\hline 2 & 3 & diftucụito & 8 & 0 & 8 & 8 & : & : & 8 & 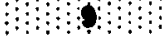 \\
\hline 2 & 1 & $\dot{T}$ & 1 & 1 & 1 & 1 & 1 & 1 & 1 & 1 \\
\hline 2 & 1 & E & 1 & 1 & 1 & 1 & 1 & 1 & 1 & 1 \\
\hline 2 & 1 & $\mathbf{S}$ & 1 & 1 & 1 & 1 & 1 & 1 & 1 & 1 \\
\hline 2 & 2 & $\mathbf{T}$ & 1 & 1 & 1 & 2 & 1 & 1 & 1 & 1 \\
\hline 2 & 2 & $E$ & 2 & 2 & 2 & 3 & 2 & 1 & 2 & 1 \\
\hline 2 & 2 & $\mathbf{s}$ & 2 & 1 & 1 & 2 & 2 & 2 & 1 & 1 \\
\hline 2 & 3 & $\mathbf{T}$ & 2 & 1 & 2 & 2 & 2 & 3 & 2 & 3 \\
\hline 2 & 3 & E & 3 & 3 & 3 & 3 & 3 & 3 & 3 & 3 \\
\hline 2 & 3 & $\mathbf{s}$ & 3 & 3 & 3 & 3 & 2 & 2 & 3 & 3 \\
\hline
\end{tabular}




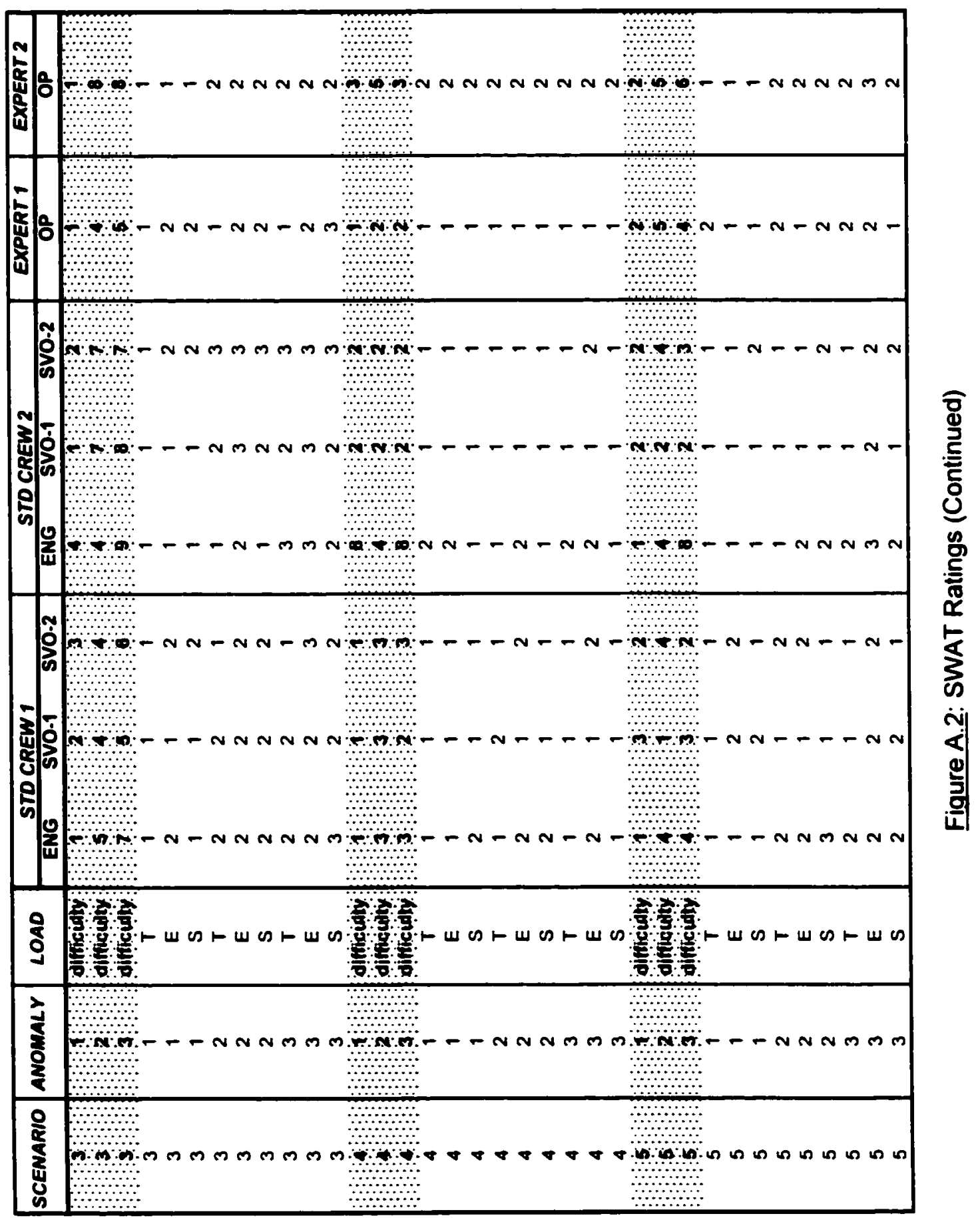




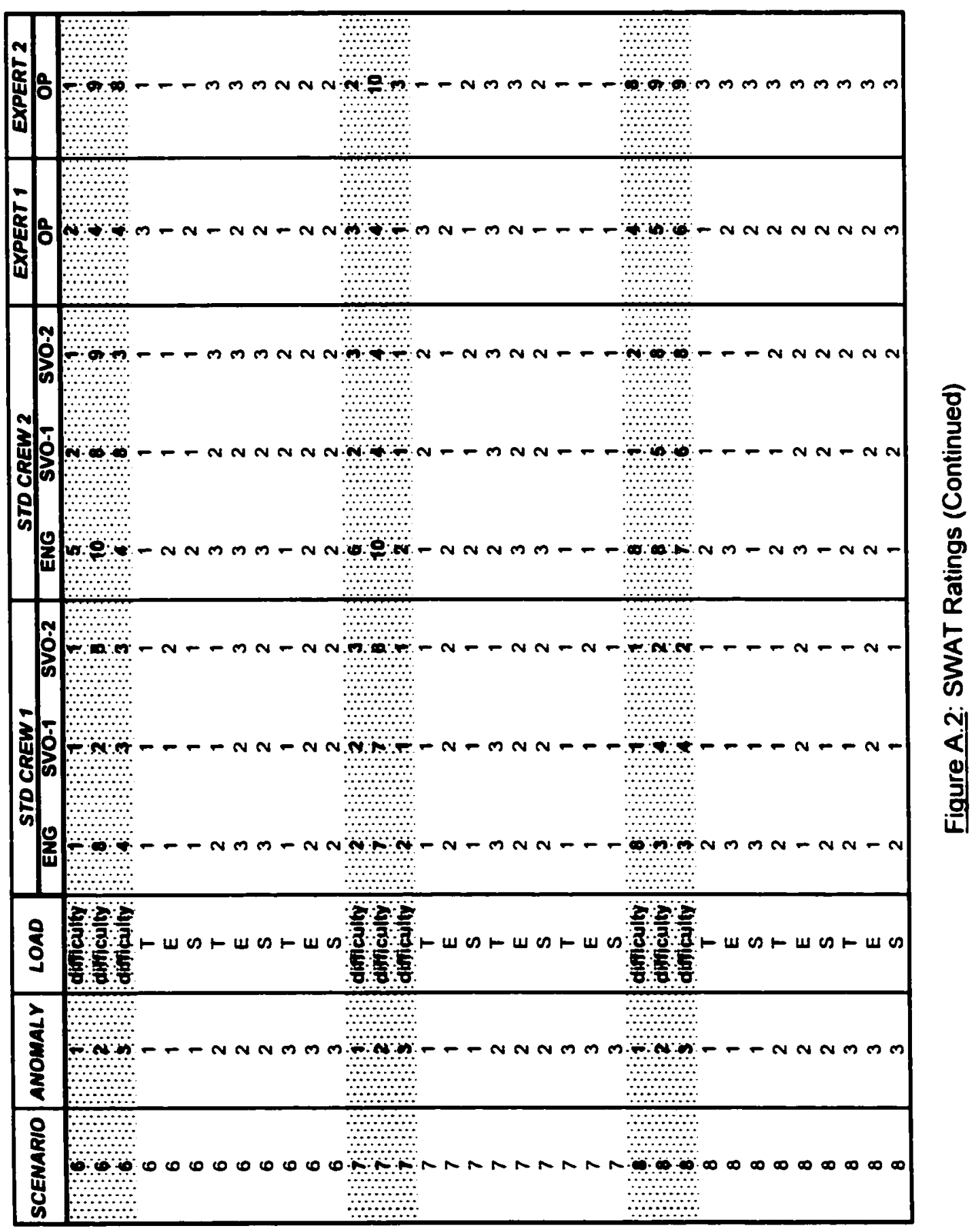




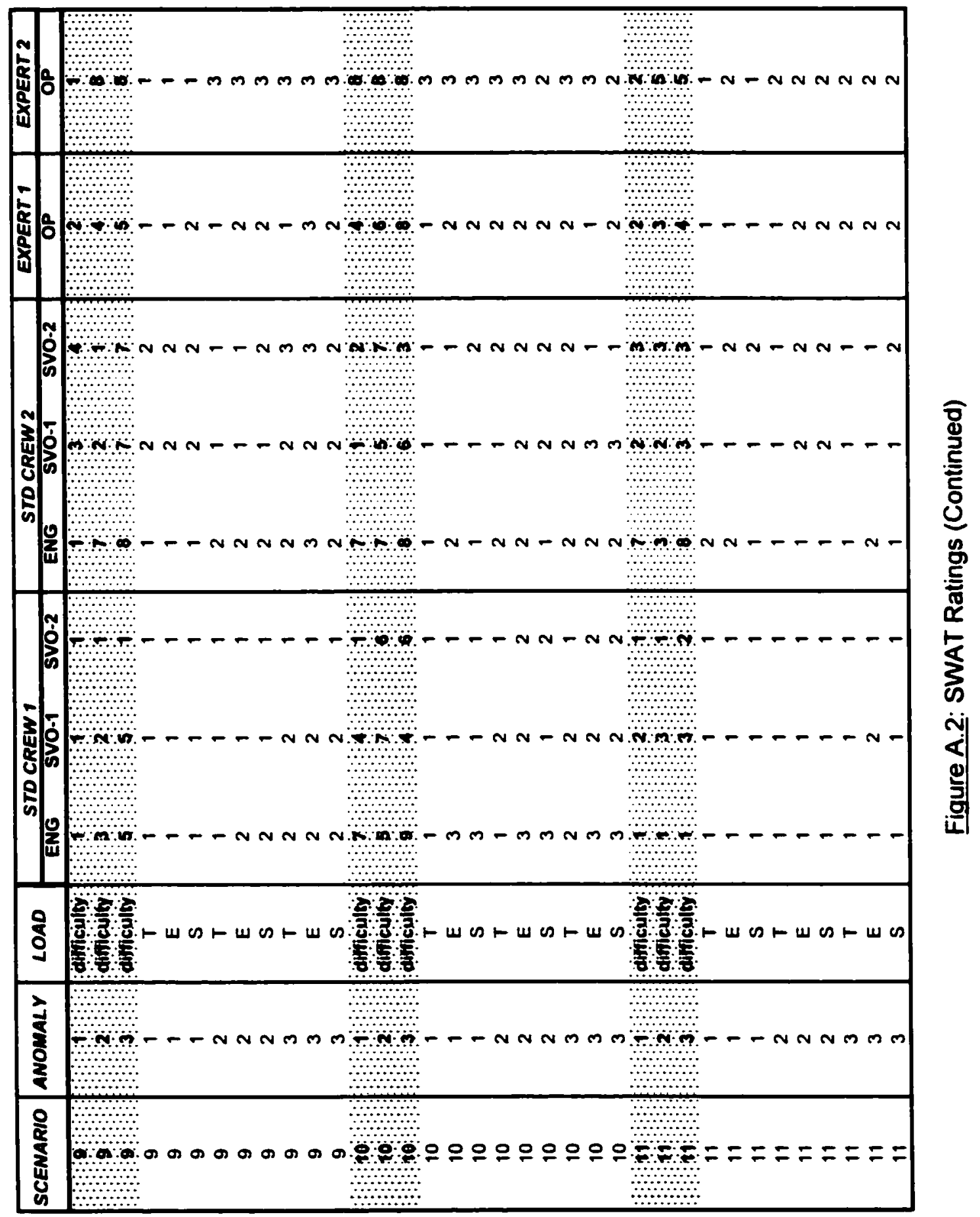




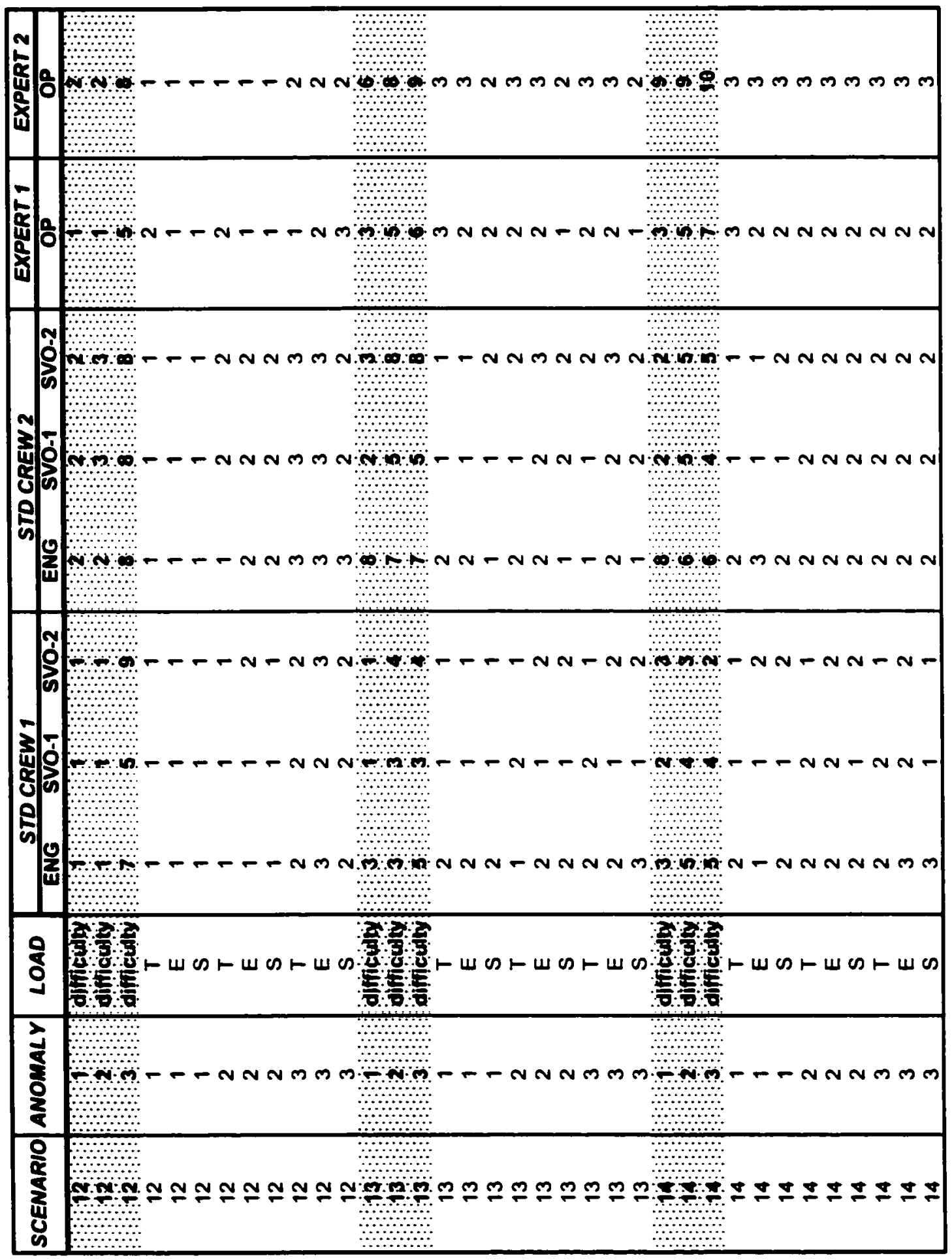




\begin{tabular}{|c|c|c|c|c|c|c|c|c|c|c|}
\hline \multirow{2}{*}{ SCENARIO } & \multirow{2}{*}{ ANOMALY } & \multirow{2}{*}{ LOAD } & \multicolumn{3}{|c|}{ STD CREW 1} & \multicolumn{3}{|c|}{ STO CREW 2} & \multirow{2}{*}{$\begin{array}{c}\text { EXPERT } 1 \\
\text { OP }\end{array}$} & \multirow{2}{*}{$\frac{\text { EXPERT } 2}{\text { OP }}$} \\
\hline & & & ENG & SV0-1 & SVO-2 & ENG & SV0-1 & SV0-2 & & \\
\hline :T5: & $1:$ & :dpiciuly: & $\vdots: 2 \vdots$ & $0: \div: \vdots$ & : & $8:$ & 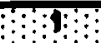 & : & 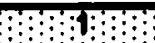 & 和: \\
\hline 18 & : & didicuity & 3 & 4 & 3 & 2 & 2 & $x$ & :3: & 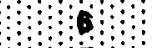 \\
\hline 19 & 3 & dinticiulis & 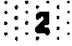 & 3 & 1 & 8 & 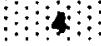 & 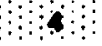 & : & 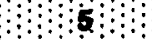 \\
\hline 15 & $i$ & $\mathbf{T}$ & $i$ & 1 & $\because$ & $\because$ & 1 & 1 & $\because$ & 2 \\
\hline 15 & 1 & $\mathbf{E}$ & 1 & 1 & 1 & 2 & 1 & 1 & 1 & 2 \\
\hline 15 & 1 & $\mathbf{S}$ & 2 & 1 & 1 & 2 & 1 & 1 & 1 & 2 \\
\hline 15 & 2 & $\mathbf{T}$ & 1 & 1 & 1 & 1 & 1 & 1 & 1 & 2 \\
\hline 15 & 2 & $\mathbf{E}$ & 1 & 2 & 2 & 1 & 2 & 1 & 1 & 3 \\
\hline 15 & 2 & s & 1 & 1 & 1 & 1 & 1 & 1 & 1 & 2 \\
\hline 15 & 3 & $\boldsymbol{T}$ & 1 & $i$ & 1 & 2 & 1 & 1 & 1 & 2 \\
\hline 15 & 3 & E & $i$ & 1 & 2 & 2 & 2 & 2 & 1 & 2 \\
\hline 15 & 3 & s & 2 & 1 & 2 & 2 & 1 & 1 & 1 & 2 \\
\hline
\end{tabular}

Figure A.2: SWAT Ratings (Continued) 
Figure A.3: Complete SWAT Analysis

\author{
8 SUBJECTS
}

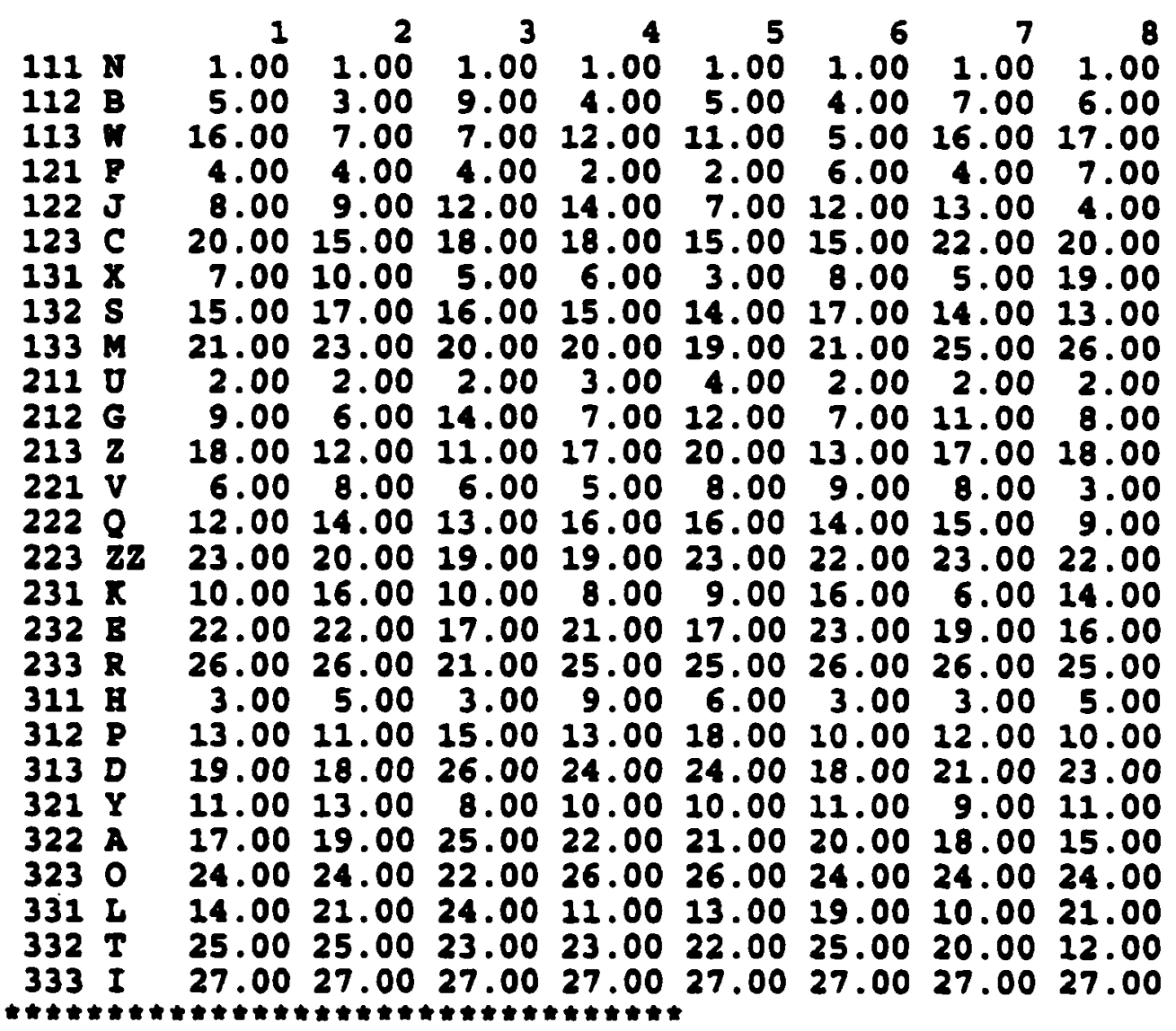


** PROTOTYPB aNALYSIS OP BACH SUBJECTS DATA ** THB RENDALL'S COBFEIBIBNT OF CONCORDANCB WAS: $W=.8831$ SPRARMAN RANK CORRBLATION (RS) FOR BACH SUBJBCT

\begin{tabular}{|c|c|c|c|c|c|c|}
\hline $\begin{array}{c}\text { aub. } \\
1 \\
2 \\
3 \\
4 \\
5 \\
6 \\
7 \\
8\end{array}$ & $\begin{array}{l}\text { TRS } \\
.54 \\
.69 \\
.65 \\
.60 \\
.66 \\
.65 \\
.41 \\
.42\end{array}$ & $\begin{array}{l}\text { TS8 } \\
.61 \\
.65 \\
.69 \\
.68 \\
.77 \\
.62 \\
.52 \\
.46\end{array}$ & $\begin{array}{l}\text { ETS } \\
.63 \\
.86 \\
.63 \\
.58 \\
.51 \\
.84 \\
.50 \\
.59\end{array}$ & $\begin{array}{l}8 S T \\
.73 \\
.88 \\
.66 \\
.65 \\
.56 \\
.87 \\
.64 \\
.70\end{array}$ & $\begin{array}{l}\text { SET } \\
.93 \\
.77 \\
.78 \\
.90 \\
.88 \\
.79 \\
.98 \\
.83\end{array}$ & $\begin{array}{l}\text { STB } \\
.90 \\
.72 \\
.79 \\
.90 \\
.93 \\
.72 \\
.95 \\
.77\end{array}$ \\
\hline
\end{tabular}


PIOT OR ORIGIAR DATA (X-AXIS) VS. DISPARITIBS ( $Y$-AXIS)

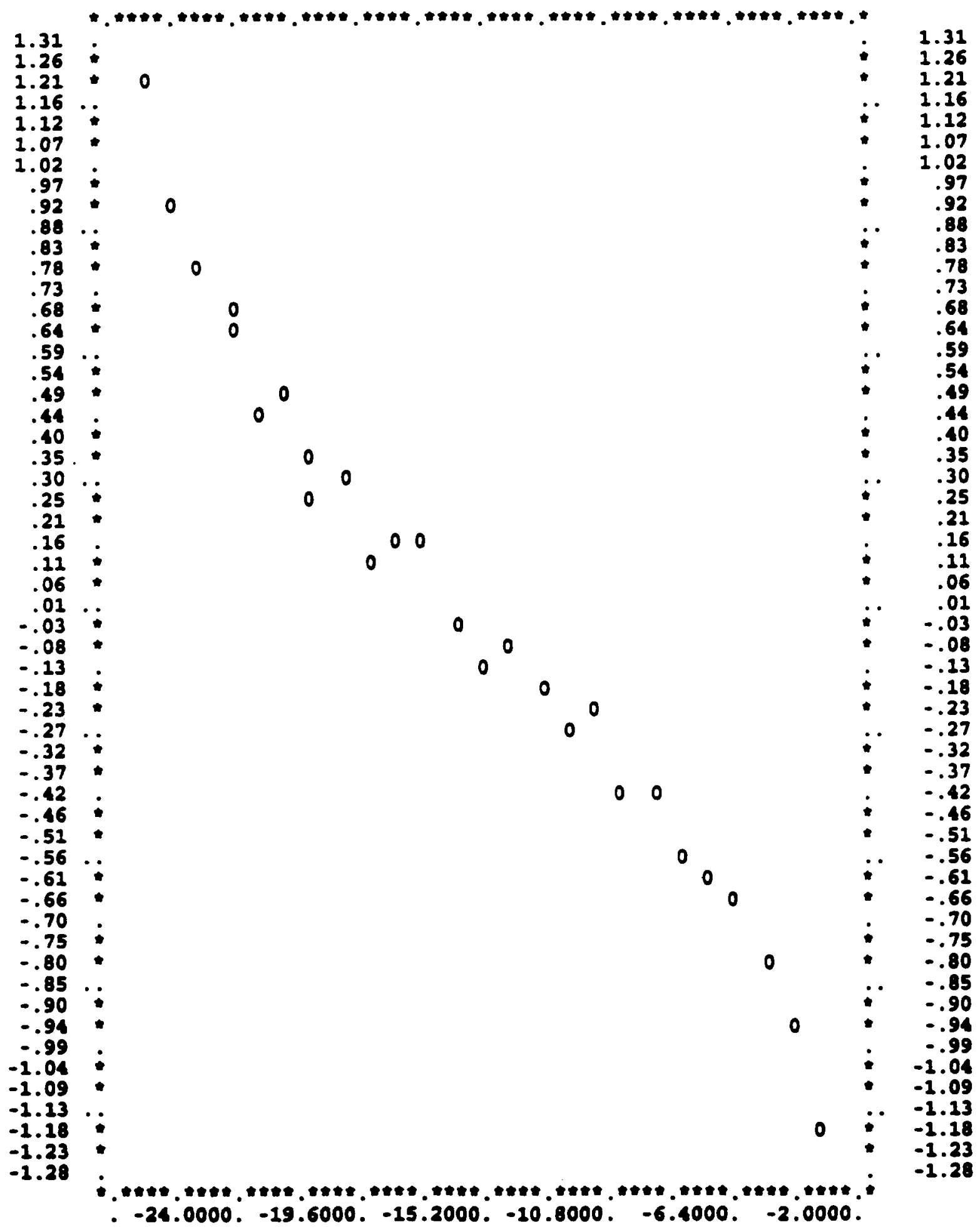


Matrix Block No.

$T$

$=1$

- 2

1.00

7.00

19.00

$=3$ $\begin{array}{rr}1 & 2 \\ 2.00 & 5.00 \\ 3.00 & 11.00\end{array}$

14.0021 .00

$S=1$

3

$\begin{array}{rrrrrr}r & & & & s=2 \\ =1 & 6.00 & 8.00 & 10.00 & 3 & \end{array}$

$\begin{array}{llll}T & = & 1 & 2 \\ =1 & 17.00 & 18.00 & 23.00 \\ =2 & 20.00 & 22.00 & 24.00 \\ =3 & 26.00 & 25.00 & 27.00\end{array}$

Test Sumary Statistics: Independence.

$T$ independent of $B$ ND $S$

The values printed below indicate the degree to which the axioms are being flt by the data. The SIGIIP columing indicate the observed z-value in testing the null bypothests that the obnerved and expected error proportions are equal. Then no z-value is given, (i.e., only ${ }^{\prime}$ ), it Indicates an inoufficient number of observations to do the hypothesis teat.

NUMBER PROPORTN PROPORTA SIGNIF OBSERVED BXPBCTED

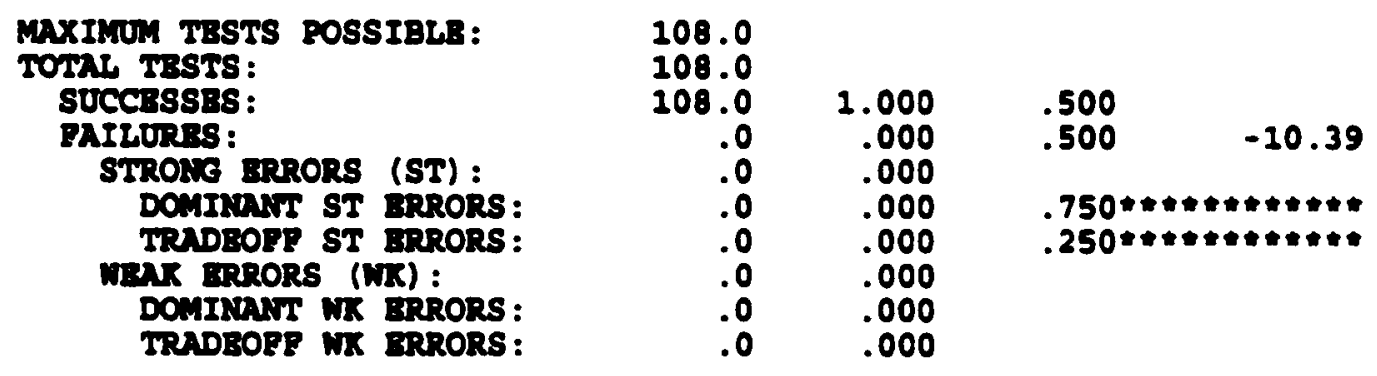


Independence: Factor $S$ is the outside factor.

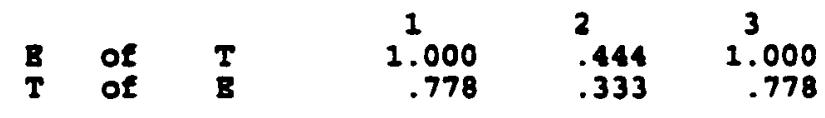

Test Summary Statistics: Independence.

$B$ independent of $T$ Nid $\mathbf{s}$

The values printed below indicate the degree to which the axioms are being it by the data. The SIGIIP colume indicate the observed z-value in testing the null hypothenis that the obcerved and expected error proportion are equal. When no z-value is given, (1.e., only 'a), it indicated an inoufficient number of observations to do the hypothesis test.

MUTBRR PROPORTS PROPORTS SIGNIP OBSERVT EXPECTHD

\begin{tabular}{|c|c|c|c|}
\hline 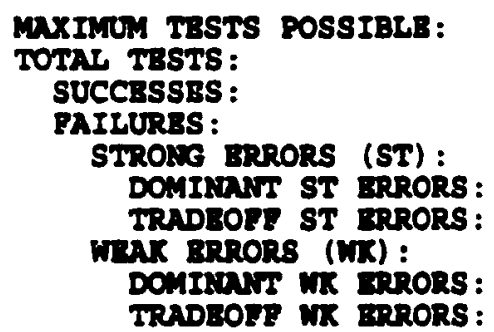 & $\begin{array}{r}108.0 \\
108.0 \\
108.0 \\
.0 \\
.0 \\
.0 \\
.0 \\
.0 \\
.0 \\
.0\end{array}$ & $\begin{array}{r}1.000 \\
.000 \\
.000 \\
.000 \\
.000 \\
.000 \\
.000 \\
.000\end{array}$ & $\begin{array}{l}.500 \\
.500 \\
.7500 .39 \\
.250 *-10.4\end{array}$ \\
\hline
\end{tabular}

Independence: Pactor $T$ is the outeide Eactor.

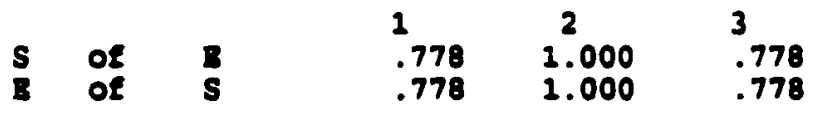

Teat Sumary Statiatice: Indopendence.

$S$ indopendent of $T$ ND $B$

The values printed below indicate the degree to which the axions are being fit by the data. The sIaisp columa indicate the observed 3-value in teeting the mull bypothenis that the observed and expected error proportion are equal. Then no z-value is given, (i.e., only 'is), it Indicates an inoufficient number of oboervations to do the hypotheols tent.

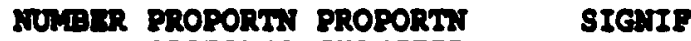
OBSTRVID ERPCLID 


\begin{tabular}{|c|c|c|c|}
\hline 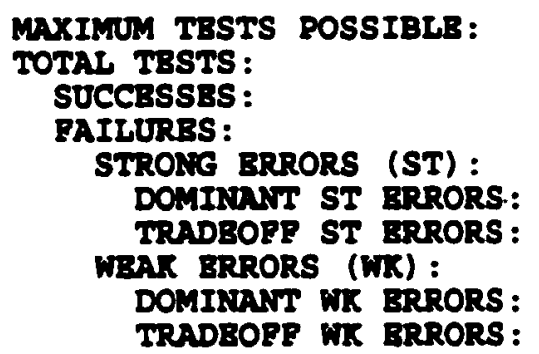 & $\begin{array}{r}108.0 \\
108.0 \\
108.0 \\
.0 \\
.0 \\
.0 \\
.0 \\
.0 \\
.0 \\
.0\end{array}$ & $\begin{array}{r}1.000 \\
.000 \\
.000 \\
.000 \\
.000 \\
.000 \\
.000 \\
.000\end{array}$ & $\begin{array}{l}.500 \\
.500 \\
.750 \\
.250 *-10.39\end{array}$ \\
\hline
\end{tabular}

Independence: Pactor $B$ is the outeide factor.

$\begin{array}{llllll}T & & 1 & 2 & 3 \\ \mathbf{S} & \text { of } & \mathbf{S} & 1.000 & .778 & .111 \\ \mathbf{T} & 1.000 & .778 & .778\end{array}$

\begin{abstract}
Test Sumary statiotice: Double Cancellation.
Double Cancellation in $T \times \quad B$

The values printed below Indlcate the degree to which the axiows are belng fit by the data. The sICAIP columa indicate the observed a-value in testing the null hypothesis that the observed and expected error proportions are equal. When no z-value to given, (i.e., only io), it indicates an insulficient number of observations to do the hypothesis test.
\end{abstract}

\title{
NUMDER PROPORIN PROPORIY SIENIF OBSERVED EXPBCIRD
}

\begin{tabular}{|c|c|c|c|}
\hline 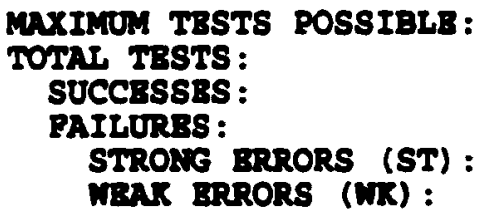 & $\begin{array}{r}3.0 \\
3.0 \\
3.0 \\
.0 \\
.0 \\
.0\end{array}$ & $\begin{array}{r}1.000 \\
.000 \\
.000 \\
.000\end{array}$ & 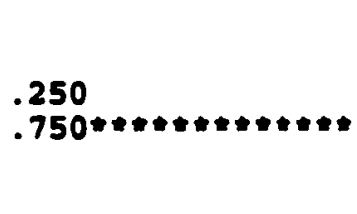 \\
\hline
\end{tabular}

\footnotetext{
Teet Sumary statietce: Double Cancellation.

Double Cancellation in $B \quad x \quad s$

NULDRR PROPORIX PROPORIT

OESERVID DXPBCTED

SICHIP
} 


\begin{tabular}{|c|c|c|c|}
\hline $\begin{array}{l}\text { MAXIMUM TRSTS ROSSIBLR : } \\
\text { TOTAL TESTS : } \\
\text { SUCCRSSES : } \\
\text { BAILURBS : } \\
\text { STRONG BRRORS (ST) : } \\
\text { WRAK BRRORS (WK) : }\end{array}$ & $\begin{array}{r}3.0 \\
3.0 \\
3.0 \\
.0 \\
.0 \\
.0\end{array}$ & $\begin{array}{r}1.000 \\
.000 \\
.000 \\
.000\end{array}$ & $\begin{array}{l}.250 \\
.750+t+t+t+t+t+t+t\end{array}$ \\
\hline
\end{tabular}

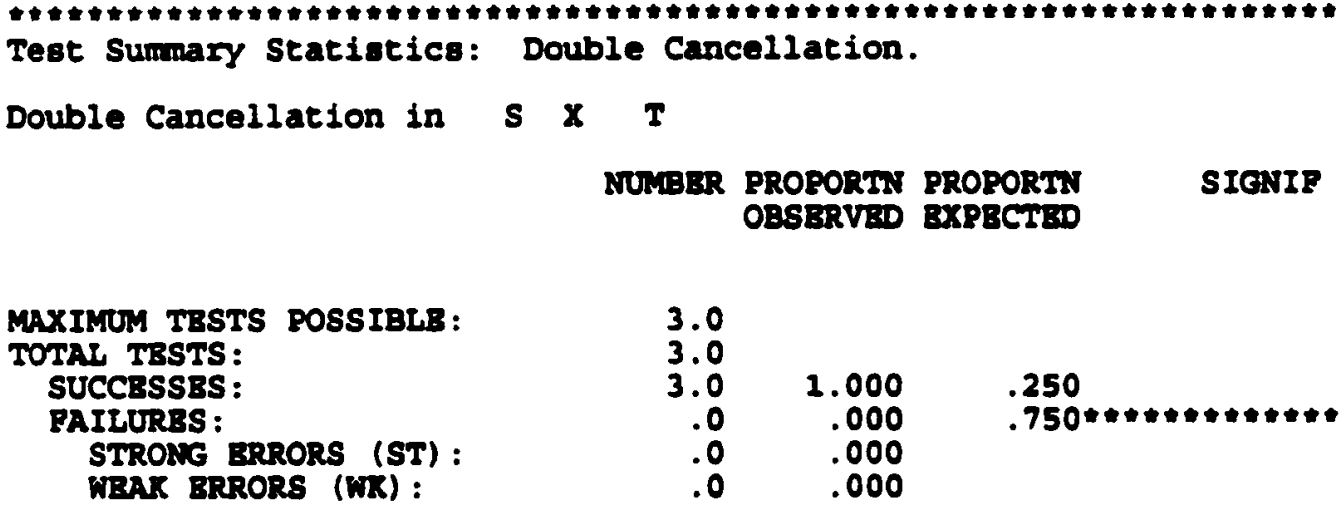

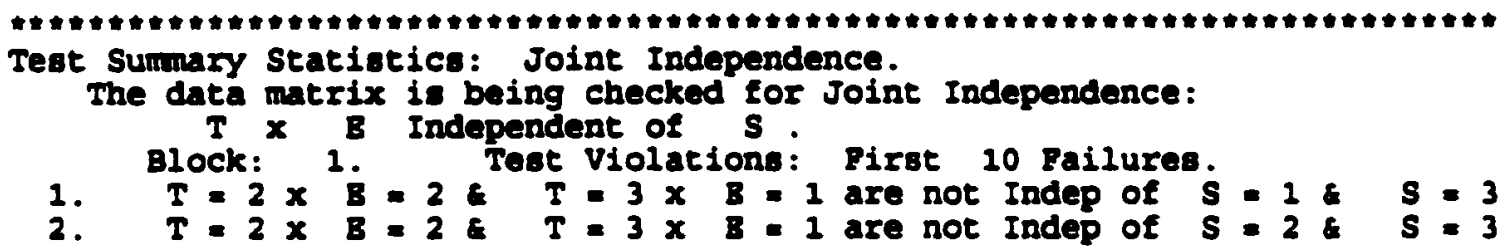

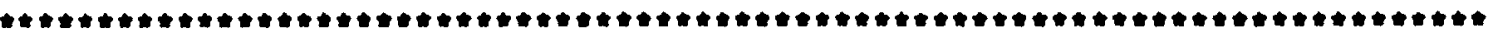
Teat Sumary Statistice: Joint Independence.

\section{T $\mathrm{X} B$ independent of $\mathrm{S}$}

The values printed below indicate the degree to which the axion are being fit by the data. The sIGNIF colume indicate the obrerved z-value in tenting the null hypothesis that the obeerved and expected exror proportion are equal. When no z-value is given, (i.e., aniy.'s), it indicates an inoufficient number of obeervations to do the hypothesio test.

NOHDER PROPORIN PROPORIX SIENIE OBSERVID EXPECH.1D 


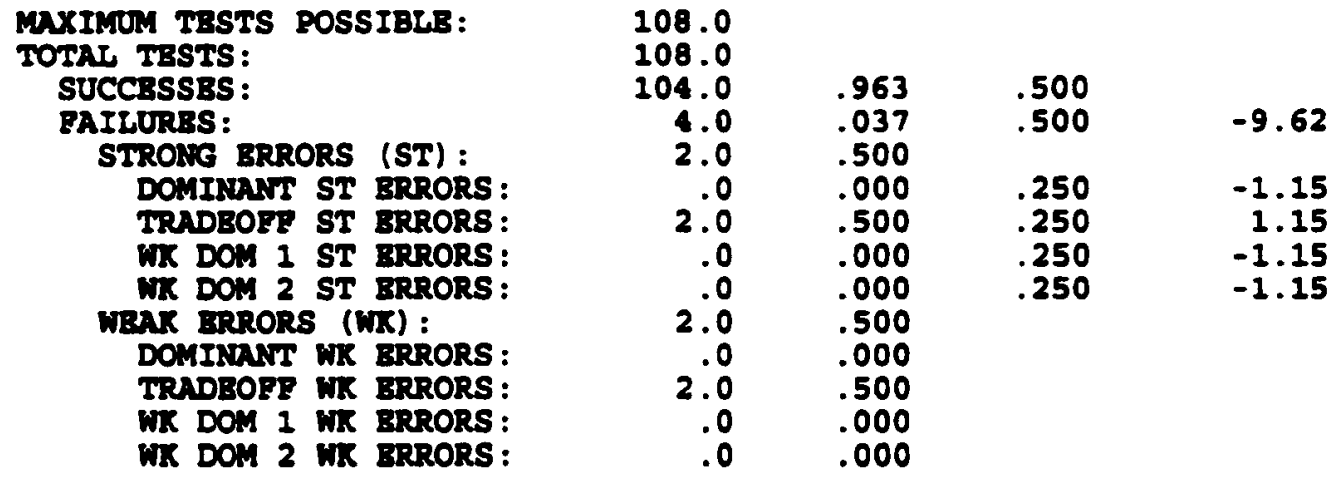

Joint-Independence: Bactor $s$ is the outside Eactor.

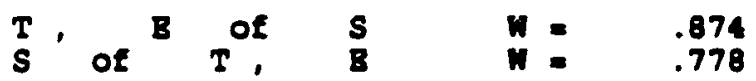

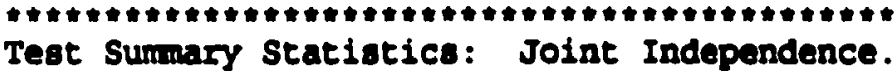

B $X$ S Independent of $T$

The valuee printed below indicate the degree to which the axious are being $\mathrm{E}$ tt by the data. The SIGIIF columes indicate the observed z-value in testing the null hypothesis that the observed and expected error proportions are equal. When no z-value is given, (1.e., only $*$ 's), it indicates an ineufficlent number of observations to do the bypothesis test.

NUMBER PROPORTN PROPORTN SIGNIE OBSERVED BXPECTEDD

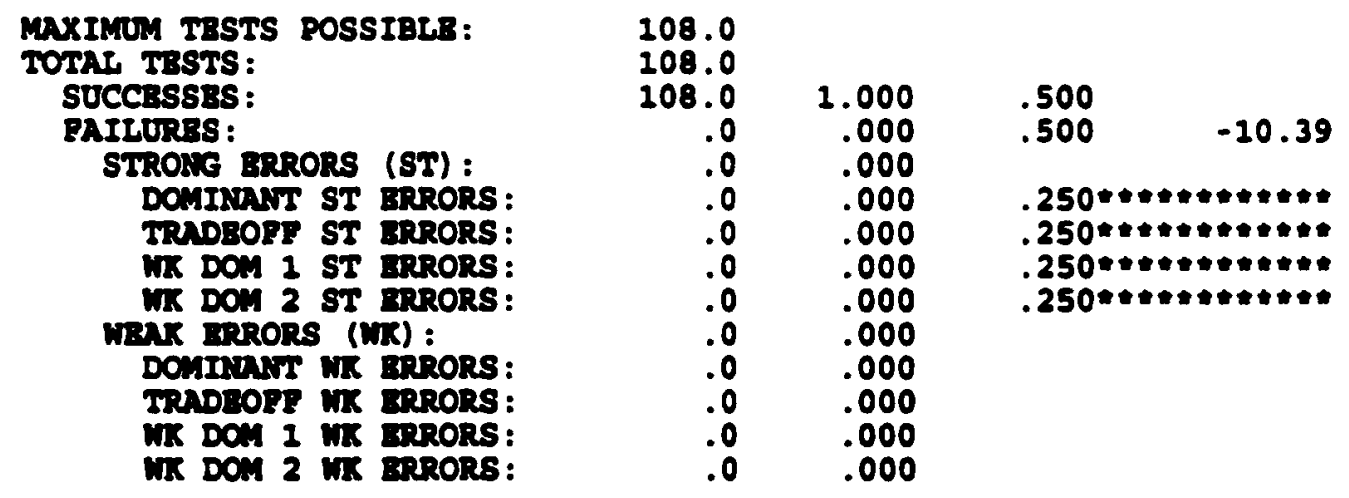


Joint-Independence: Ractor $T$ is the outside factor.

$$
\begin{array}{lllll}
B & S \text { of } & T & W= & .933 \\
T & B & S & W & =481
\end{array}
$$

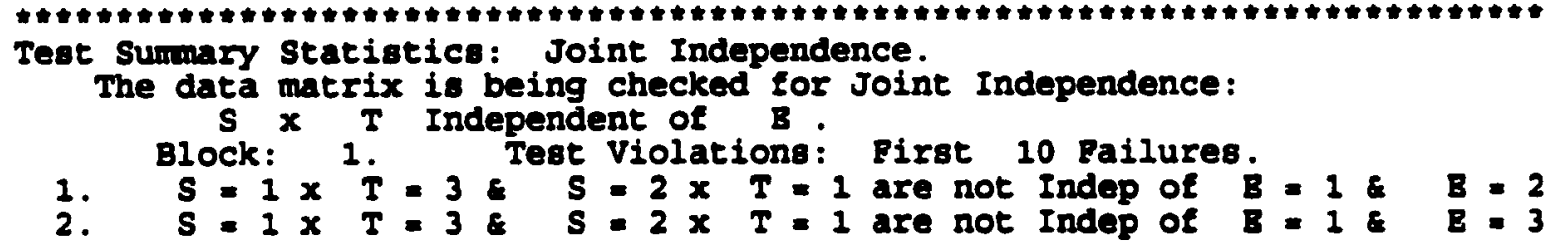

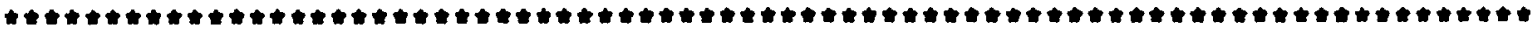

Test Sumary Statistice: Joint Independence.

\section{$S \mathrm{X}$ T independent of $B$}

The values printed below indicate the degree to which the axions are being fit by the data. The SICNIP columa indicate the observed z-value in testing the null hypothesis that the observed and expected error proportions are equal. When no z-value is given, (1.e., only 's), it indicates an ingufficient number of observations to do the hypothesis test.

\section{IUNBBRR PROPORTN PROPORTN SIGNIE OBSBRVED EXPBCTED}

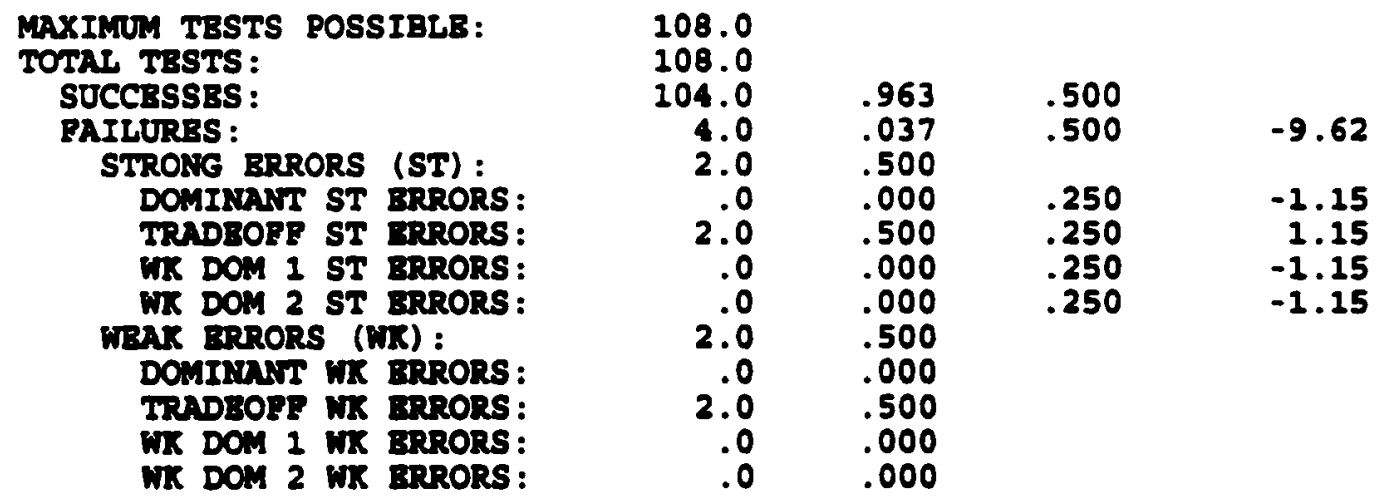

Joint-Independence: Factor $B$ is the outside Eactor.

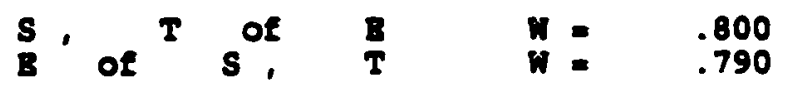




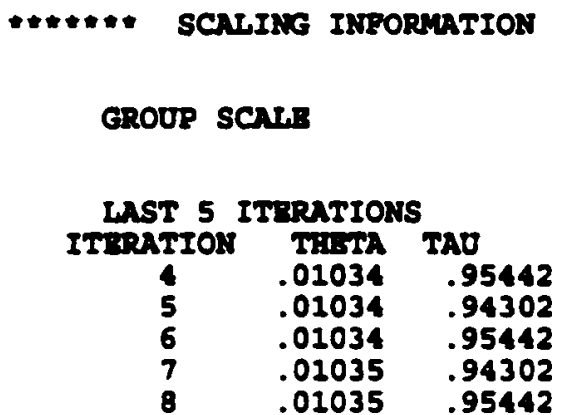

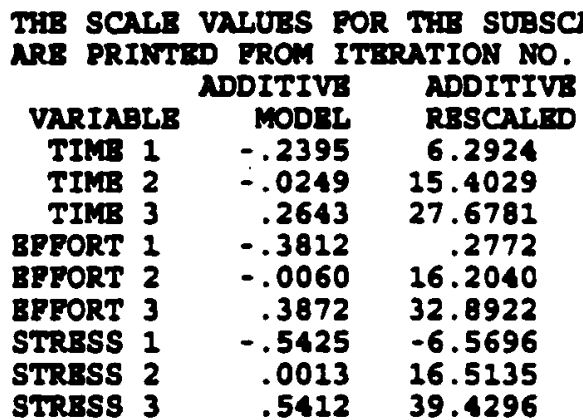

\section{APPROXIMATS RBLATIVB IMPORTANCB OP BACA PACTOR}

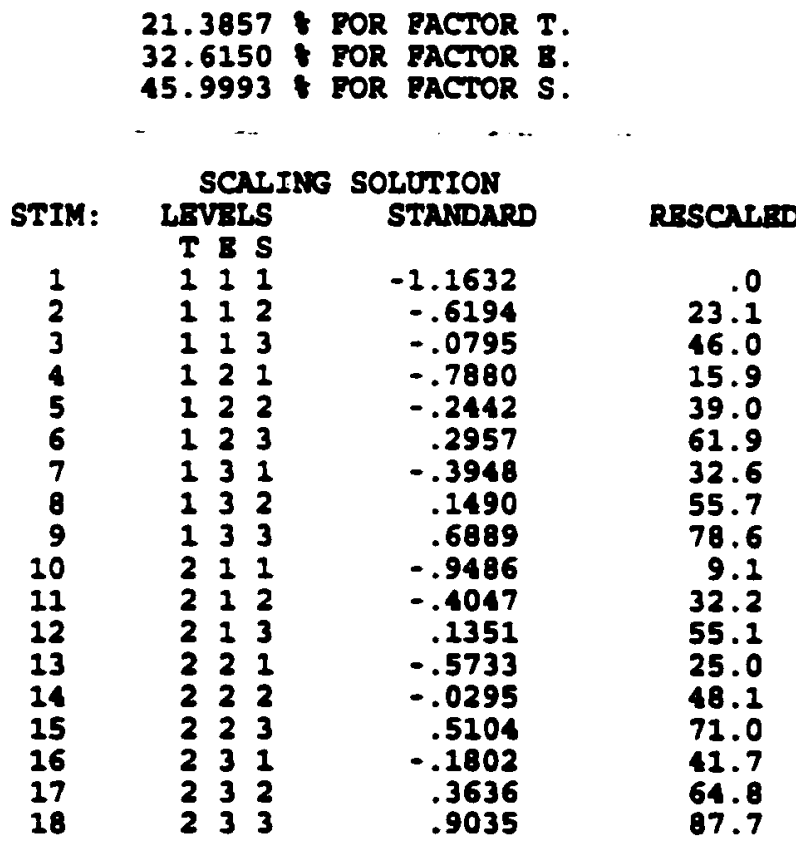




$\begin{array}{rrrrrr}19 & 3 & 1 & 1 & -.6594 & 21.4 \\ 20 & 3 & 2 & -.1155 & 44.5 \\ 21 & 3 & 3 & .6243 & 67.4 \\ 22 & 3 & 2 & 1 & -.2841 & 37.3 \\ 23 & 3 & 2 & .2597 & 60.4 \\ 24 & 3 & 3 & .7996 & 83.3 \\ 25 & 3 & 3 & 1 & .1090 & 54.0 \\ 26 & 3 & 3 & 2 & .6528 & 77.1 \\ 27 & 3 & 3 & 3 & 1.2927 & 100.0\end{array}$




\section{Appendix B}

\section{INSTITUTIONAL REVIEW BOARD (IRB) APPROVAL FOR USE OF HUMAN SUBJECTS}

This appendix includes the package submitted to the Institution Review Board (IRB) and the necessary approval documentation. 

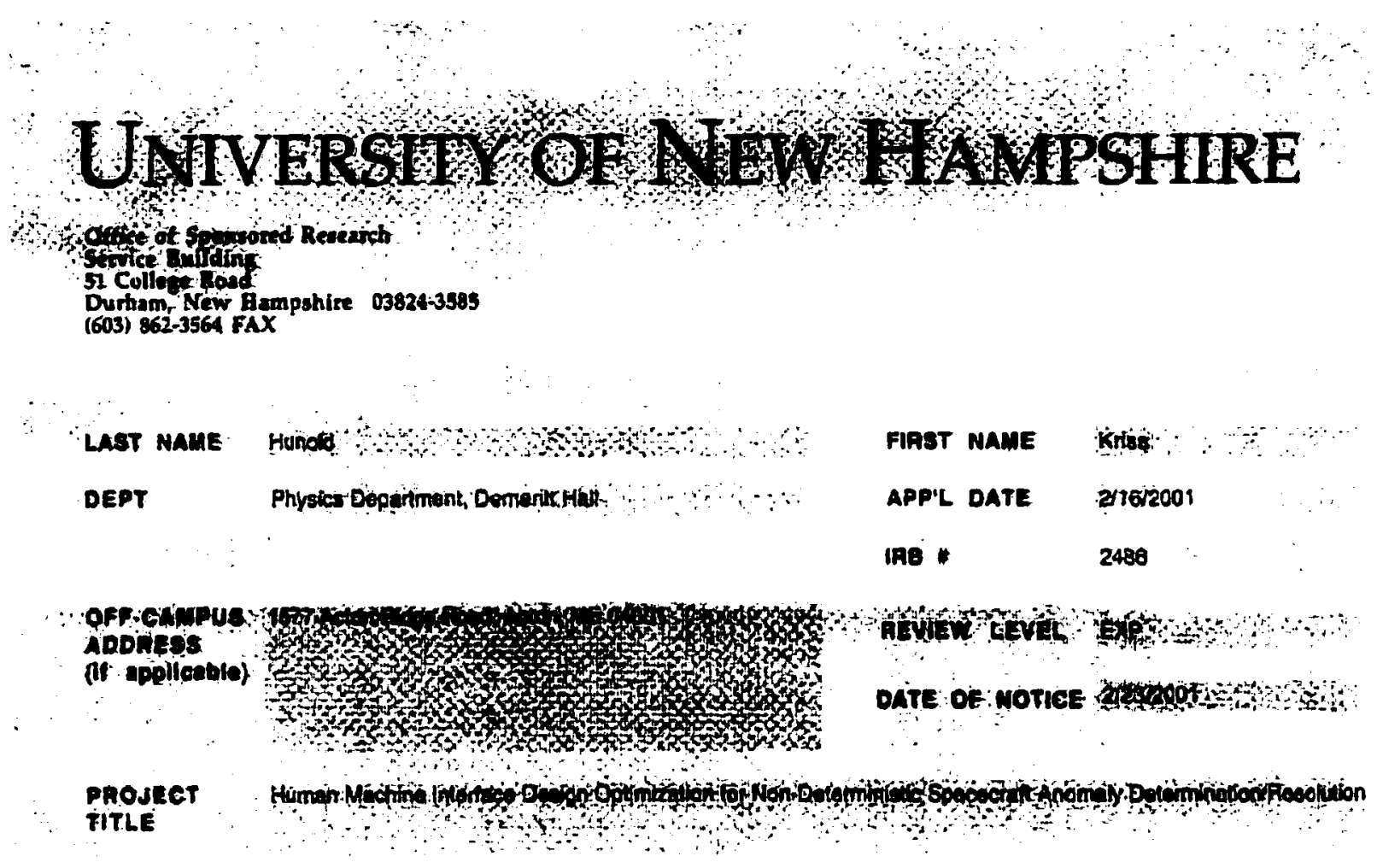

The Insultutionat Reviow Boand (IRB) for the Protection of Human Subjects in Research has reviewed the protocol for your project as Expodied as described in Fedtral Pegulations 45 CFA 46. Subsection 46.110 (b) calogory 7.

Approval is granted for one your trom the epproval chte above. At the end of the epproval cortod you wh be ackedto

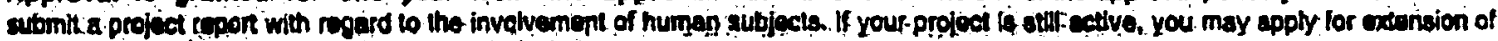
IfR upproval thiough ints ofrice.

The protection of human subjects in your, study is an ongoing process for wnich you hold primary responsibilty. In receiring IRB approval-for your protocol, you agree to conduct the project in accordance with the eithical principles and guldefines for the protecton

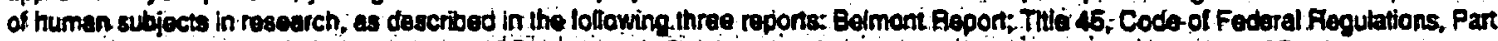

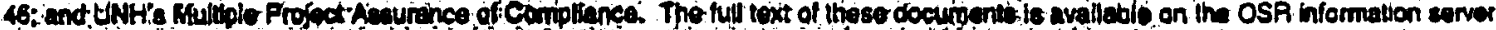

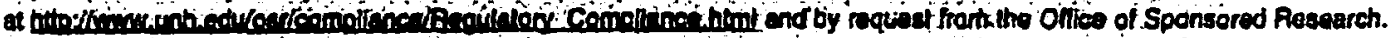

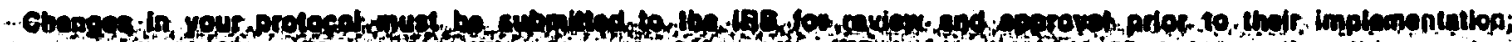

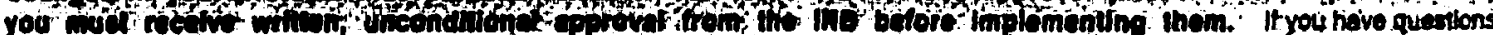

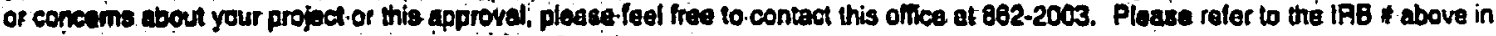
all correspondence relaled to this project. The iRB wishes you success with your research.

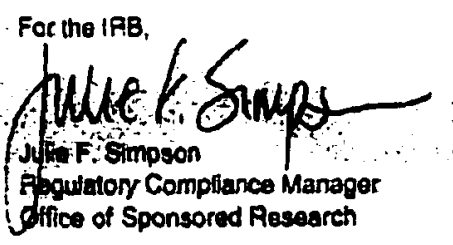

ca: File

Dr. John LiCaurse. Electrical a Computer Engineosigg poppi:

Fiqure B.1: Institutional Review Board Approval Letter 


\section{Appendix C}

\section{CREW SUBJECTIVE SURVEYS}

This appendix contains the sample surveys completed by the crews, as well as the SWAT card sort data sheets. 
I. Please identify the three satellite anomalies that were presented in this scenario:

1.

3.

II. Please circle a number corresponding to what you thought the difficulty level was of each of the three tasks you were to complete

\begin{tabular}{|c|c|c|c|c|c|c|c|c|c|}
\hline \multicolumn{3}{|c|}{ Low difficulty } & \multicolumn{4}{|c|}{ Moderate Difficulty } & \multirow[b]{2}{*}{8} & \multirow[b]{2}{*}{9} & \multirow{2}{*}{$\begin{array}{l}\text { High Difiliculty } \\
10\end{array}$} \\
\hline 1 & 2 & 3 & 4 & $\mathbf{5}$ & 6 & 7 & & & \\
\hline \multicolumn{10}{|c|}{ Anomaly \#2 } \\
\hline \multicolumn{3}{|c|}{ Low difficulty } & \multicolumn{4}{|c|}{ Moderate Difficulty } & & & High Difficutty \\
\hline 1 & 2 & 3 & 4 & 5 & 6 & 7 & 8 & 9 & 10 \\
\hline \multicolumn{10}{|c|}{ Anomaly 43} \\
\hline \multicolumn{3}{|c|}{ Low difficulty } & \multicolumn{4}{|c|}{ Moderate Dimleulty } & & & High Difficulty \\
\hline 1 & 2 & 3 & 4 & 5 & 6 & 7 & 8 & 9 & 10 \\
\hline
\end{tabular}

III. Subjective Workload Assessment Technique (SWAT) Ratings: Please rate each anomaly according to the SWAT Rating scale:

Time Laed - Roting Scale Definitions

Onen have spare time interruptions $\alpha$ overtap among actsvities $\propto c a r$ intrequently or not at al

2 Occastonally have spare tume. Interruptions or overtap among activities occur trequently

3 Almost never have spare time intertuptions or overiap among activities are very frequent. or oceur all the ume

\section{Mental Effort Loed - Rating Scale Dafinitions}

- Very hitle conscrous effor or concentration required. Actrvity is almost automatic. requinng intie attemion

2 Mocerate conscious mental effort or concentration fequred Complexty of activity is moderately high owe to uncertanty unpredictability, or unfamilianty Considerable attemion requared

3 Extenswe mental eftort and concentration are nocessary. Very complex activity requaring lotal attention

\section{Paychological Streas Laed - Rading Scele Dafinitions}

1 Litte confusion. nsk. frustration, $\propto$ arwety exists and can De sesily accommocated

2. Moderate stress die to contusion. inutration. or anxuety noticeabla adds to workload. Signficant compensation is required to mantain acequate pertormence.

3 High to very intense stress oue 10 contusion, frustration. $\alpha$ anxiety High to extreme determination and seff-control required.

Anomaly:1:

- Time Load (1, 2. or 3)

- Mental Effort Load (1, 2, or 3)

- Psychological Stress Load (1, 2, or 3)

Anomaly :?:

- Time Load (1, 2, or 3)

- Mental Effor Load (1, 2, or 3)

- Psychological Stress Load (1, 2, or 3)

Anomaly 1 :

- Time Load (1. 2. or 3)

- Mental Effort Load (1, 2, or 3)

- Psychological Stress Load (1, 2, or 3) enter 1,2, or 3 for each

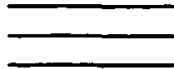

enter 1,2,or 3 forench

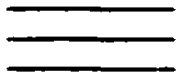

enter 1,2, or 3 toreach

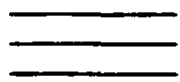

Fiqure C.1: Subjective Measures Worksheet 


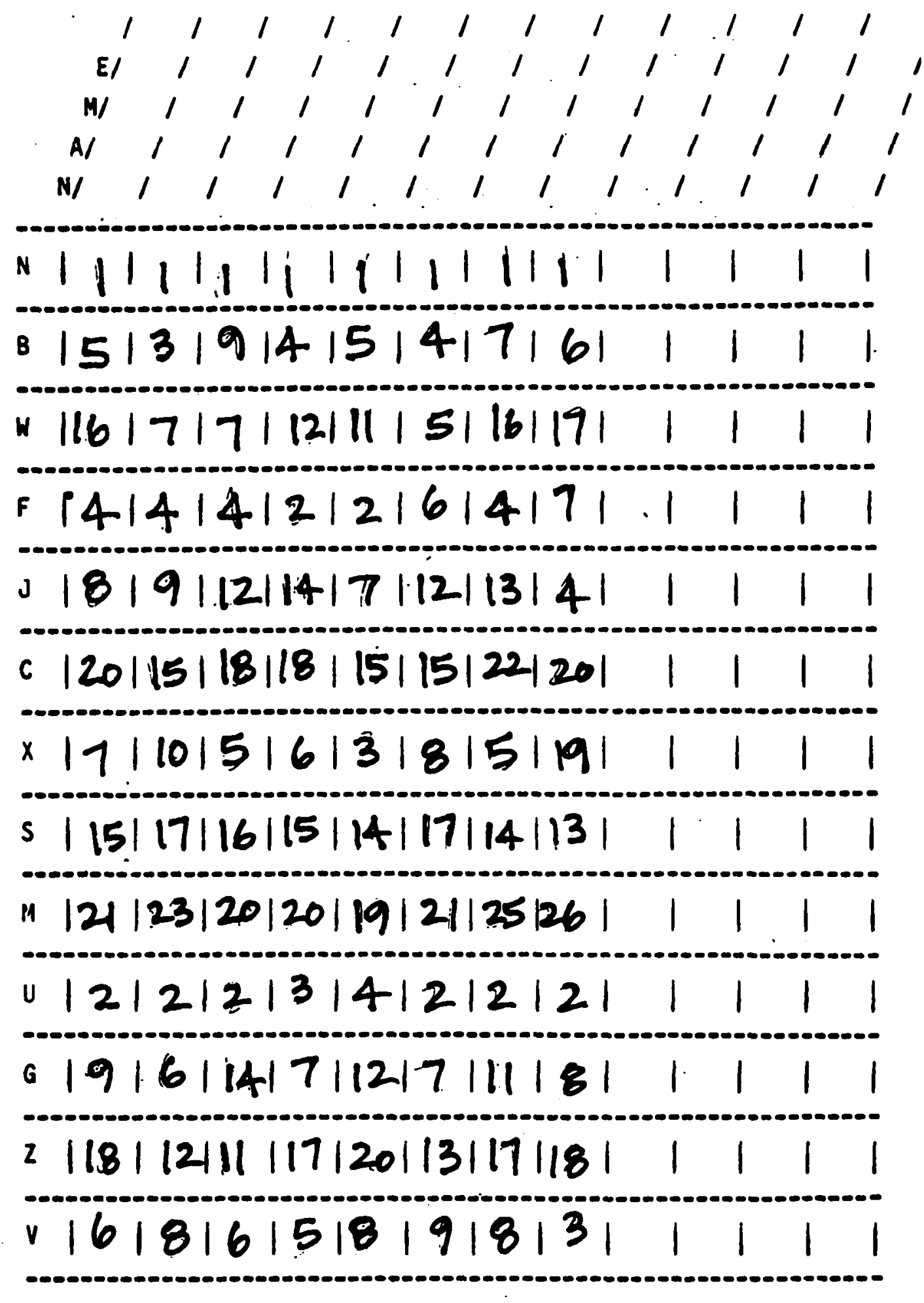

Figure C.2: SWAT Card Sort Data Sheet

161

Reproduced with permission of the copyright owner. Further reproduction prohibited without permission. 


\section{SWAT CARD SORT DATA SHEET (continued)}

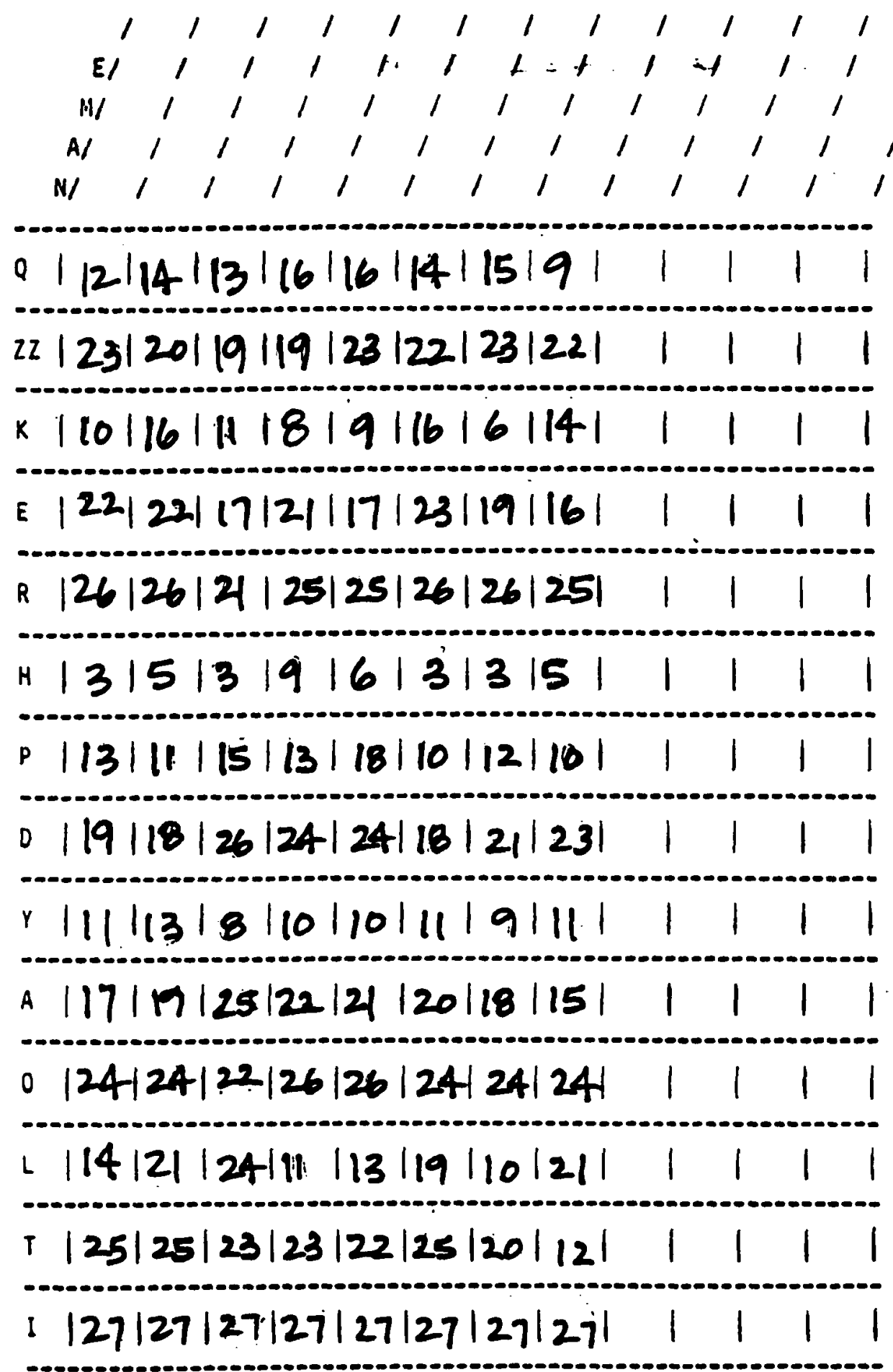

Figure C.2: SWAT Card Sort Data Sheet (Continued) 


\section{Appendix D}

\section{SAMPLE SCENARIO FORMAT}

This appendix contains a sample of the pre-brief, scenario, and out-brief administered to each crew. 


\section{INITIAL EVALUATION PREBRIEF}

INTRODUCTION: The purpose of this briefing is to acquaint you with our evaluation procedures. Please listen carefully and feel free to ask questions at any time. If anyone is feeling ill or feels they cannot participate, please let me know at this time.

PARTICIPANT INTRODUCTION: Chad Oster and I will be administering this scenario. Please refer any questions to either of us.

INVESTIGATORJPARTICIPANT RELATIONSHIP: We are here to observe and evaluate your performance. We will not provide instruction or assist you in any way while you perform your assigned duties, with exception of input clarification or vehicle response. You are expected to perform all actions as you would on duty as a mission ready crew member, so act as you would on a real world shift with real world supports. Always complete an action unless we state "action complete". Make sure you understand which action is considered complete. Be sure to speak loudly and clearly so we are aware of all actions you are performing. If you do not understand an input or stimulus. ask your evaluator to clarify the situation. Please note, we will be writing in our notebook(s) throughout the evaluation - this is strictly for recording activities and time required to complete the necessary actions.

We would also like to request refraining from what could be called "excessive behavior" (for example. abnormal levels of drinking, smoking, or exercise) during the scenario periods. This can skew your performance and affect the outcome of your success as a crew.

METHODS USED TO INITIATE EVENTS: Stimuli for this scenario may be presented in the following ways:

- Verbal input from us acting as an RTS

- Simulator/Emulator satellite contacts

- Verbal representation of satellite status in the even of a simulator malfunction

Prioritize all stimuli as if it were real world. Please clearly announce your anomaly resolution priority clearly so that we may accurately record your actions.

SAFETY: Safety is of the utmost importance! Do not take any actions which endanger human life or cause injury. Within the simulation area please be aware of (safety hazards in the evaluation area) or There are no safety hazards in the area that we are aware of.

RESPONSIBILITIES DURING ACTUAL EMERGENCIES OR MALFUNCTIONS: We will STOP or terminate a scenario if a real world emergency or malfunction interferes with the evaluation.

OPSEC/COMSEC: Proper OPSEC/COMSEC procedures must be followed at all times. We will not simulate any classified actions. If you have concerns that a particular action will lead you to breaching an unclassified environment, please stop the scenario at once and bring it immediately to our attention.

CREW SUPPORT: During this scenario, you will act as a crew. Keep in mind the scenario is to see how the crew works together to achieve a common goal: ensuring the health of the satellite.

INVOLVED AGENCIES: There will be virtually no external agencies involved with these scenarios other than communication between you and the Remote Tracking Station (RTS).

CLOCK ADJUSTMENTS: The scenario is set to be one hour in length; it will not be necessary to advance the clock to expedite the scenario. 
REMINDERS: Please remember this scenario is controlled material. .Do not discuss anything related to the scenario with anyone except Chad or myself.

QUESTIONS: Do you have any questions?

STARTING STATUS: Read individual scenario starting status. Crew is on a day shift in SOC-12.

\section{Pre/Post Scenario Actions:}

PRE

1. Check Tape Length

2. Configure Scenario

3. Pre-brief:

- Complete canned brief

- Remind crews there will be 3 "problems", "anomalies", or "things" to accomplish during the scenario

- Prepare crews for poor emulator presentation - encourage questions pertaining to OOL telemetry points, etc.

4. Present pass plans and starting status

5. Begin video camera (State DATE / TIME OF DAY / CREW \# / SCENARIO \#)

\section{POST}

1. Stop video

2. Thank participants

3. Subjective Survey

- Initiate survey part 1 (Anomaly Query). When complete, announce correct anomalies

- Initiate survey part 2 (SWAT) 


\section{SCENARIO FORMAT}

\section{GROUND RULES:}

- This is one-hour scenario representing an extended satellite support to provide critical mission data to warfighters in a wartime environment.

- Each scenario will be comprised of three vehicle anomalies. These anomalies are strictly limited to satellite anomalies; the ground environment will remain constant and nominal.

- Your goal is to repair, swap components, or safe the vehicle to remedy the anomalies

- You, as a crew, should coordinate the solution and any actions to be taken to complete these tasks

- Following the scenario, a subjective workload assessment task survey will be distributed to determine your perceived difficulty of each task presented in the scenario.

- The focus of task completion should be accuracy and timeliness. This is the data that will be noted and recorded during the scenario.

- You will have available to you all documentation necessary, to include operator manuals, standard command plans, and vehicle technical documentation.

SCENARIO: Wartime environment.

Preface: This scenario is an unrealistic, but challenging environment where you have total and complete control of the satellite. Your main goal is to ensure critical mission data is provided to the warfighter without fail. You have been given clearance to take any action necessary to maintain mission data. This includes emergency commanding, altering typical vehicle configuration, switching to redundant components, and any other action necessary to attempt to restore optimal operation.

- You have Satellite Control Authority (SCA) over the vehicle

- Take any action necessary to maintain nominal vehicle operation

- Actions may be taken completely at your discretion

- Coordination with TRW or AEROJET is not required

- Coordination with HHQ or other DoD or Air Force Agencies is not required

\section{TAKING ACTION:}

- Clearly voice all conclusions and actions to be taken to the crewmembers. Crew consensus will be voiced through the Lead SSO/CCMDR.

- Desired vehicle responses will, for the most part, be evident. Some extraneous vehicle reactions may not be present for several reasons. These reasons include emulator limitations, or merely the inability to predict overall vehicle reaction to certain situations or anomalies.

- If there are any questions pertaining to the response of the vehicle to your actions that are not obvious, please ask 


\section{SCENARIO \# 12}

\section{NOTES:}

1. $7221220 / G T S B$

2. Enable Logon

3. Ready A

4. Select Elset E (TCMOM X3)

Nominal Star Data outside sun shutter region

- Use Level 1 Anomaly Frames to initiate TLM status:

ISAALA = YES

$A D H L A B=H I E V$

$A D L L A B=L O E V$

$A G E 1 A B=E N A$

PGGA1P $=48.2$

$P G G A 2 P=48.2$ 
Overview: Event detector is in alarm at beginning of the support. 50SWMX reports high proton activity, which may be one problem source. In addition, this activity causes bit-flips on the vehicle, affecting the plenum pump status (ENA) and impact sensor (10G vice 20G). When fixing the plenum pump state, plenum pressure reads low (PGGA1P and PGGA2P) at $\sim 48$ psi. The crew tries to pump plenum, but the pump does not work. Since there is no decrease in pressure, a leak should be ruled out. The action required is to determine that fransducer 1 is faulty and should be switched to the redundant transducer/coil (GGXDCR $=2$ ). The plenum should be pumped $(B-1)$ and SOH completed

\begin{tabular}{|c|c|c|c|c|}
\hline Typo & Description & $\begin{array}{c}\text { Crew } \\
\begin{array}{c}\text { Assessmentuaction } \\
s\end{array}\end{array}$ & Telemetry Verifiers & Commanding \\
\hline 1 & $\begin{array}{l}1.1 \text { Event Detector, Impact } \\
\text { Sensor, and Plenum Points } \\
\text { OOL: Event detector is in alarm } \\
\text { at beginning of the support. } \\
50 \text { SWMX reports high proton } \\
\text { activity, which may be the } \\
\text { problem source. In addition, } \\
\text { this activity causes bit-flips on } \\
\text { the vehicle, affecting the } \\
\text { plenum pump status (ENA) and } \\
\text { impact sensor (10G vice } 20 \mathrm{G} \text { ). }\end{array}$ & $\begin{array}{l}\text { 1.1 Perform Checklist } \\
\text { S-1 and note OOL } \\
\text { TLM points. Prioritize } \\
\text { anomalies (1. EVENT } \\
\text { Detector, 2. IMPACT } \\
\text { Sensor, and } 3 \text {. } \\
\text { Plenum) and prepare } \\
\text { command plans. }\end{array}$ & $\begin{array}{l}1.1 \\
\text { SNAP } \\
\text { ISAALA }=\text { YES } \\
\text { ADHLAB }=\text { HIEV } \\
\text { ADLLAB }=\text { LOEV } \\
\text { AGE1AB }=\text { ENA } \\
\text { PGGA1P }=48.2 \\
\text { PGGA2P }=\mathbf{4 8 . 2}\end{array}$ & $1.1 \mathrm{~N} / \mathrm{A}$ \\
\hline 1 & & $\begin{array}{l}\text { 1.2 Resolve Level } 1 \\
\text { anomaly. Event } \\
\text { detector is in alarm; } \\
\text { perform CL C-12 } \\
1.2 .1 \text { Verify TLM } \\
1.2 .2 \text { Send } \\
\text { command to clear } \\
\text { alarm }\end{array}$ & $\begin{array}{l}1.2 \text { N/A } \\
1.2 .1 \\
\text { SNAP } \\
\text { ADHLAB = HIEV } \\
\text { ADLLAB = LOEV } \\
\text { ADPCAV 5 } \\
\text { ADPCBV = OFF } \\
\text { EVL = OFF } \\
1.2 .2 \\
\text { SNAP } \\
\text { ADHLAB = NOEV } \\
\text { ADLLAB = NOEV }\end{array}$ & $\begin{array}{ll}1.2 \text { N/A } \\
1.2 .1 \text { N/A } \\
\\
\\
1.2 .2 & \\
\text { CMD } & \text { DESCRIPTION } \\
\text { C1: S 1461 } & \begin{array}{r}\text { EDE-A POWER } \\
\text { ON/EDE TEST DIS }\end{array}\end{array}$ \\
\hline
\end{tabular}




\begin{tabular}{|c|c|c|c|c|}
\hline Type & Description & Crew AssessmentActions & Telemetry Verifiers & Commanding \\
\hline 2 & $\begin{array}{l}2.1 \text { Recognize Bit } \\
\text { Flips from proton } \\
\text { event have caused } \\
\text { the anomalous } \\
\text { points: Clear } \\
\text { IMPACT Sensor and } \\
\text { pump PLENUM. } \\
\text { Realize that the } \\
\text { PUMP is in ENA } \\
\text { state, but the } \\
\text { plenum pressure is } \\
\text { low. Crew should } \\
\text { disable the pump } \\
\text { and decide course } \\
\text { of action. }\end{array}$ & $\begin{array}{l}\text { 2.1 Impact sensor: (CL C-13) } \\
\begin{array}{l}2.1 .1 \text { Verify TLM points } \\
2.1 .2 \text { Calculate necessary } \\
\text { repeat steps to clear } \\
\text { alarm. Send command } \\
\text { to clear the alarm }\end{array} \\
\begin{array}{l}\text { 2.1.3 Calculate necessary } \\
\text { steps to reset to proper setting } \\
\text { (20G). Send commands } \\
\text { necessary to reach proper } \\
\text { setting. }\end{array}\end{array}$ & $\begin{array}{l}2.1 \text { N/A } \\
2.1 .1 \\
\text { SNAP } \\
\text { ISAALA }=\text { YES } \\
\text { ISASTA = 20G } \\
2.1 .2 \\
\text { SNAP } \\
\text { ISAALA }=\text { NO } \\
\text { ISASTA = ALCLR } \\
2.1 .3 \\
\text { SNAP } \\
\text { ISASTA }=20 G \\
\text { ISABUV }=28.3\end{array}$ & 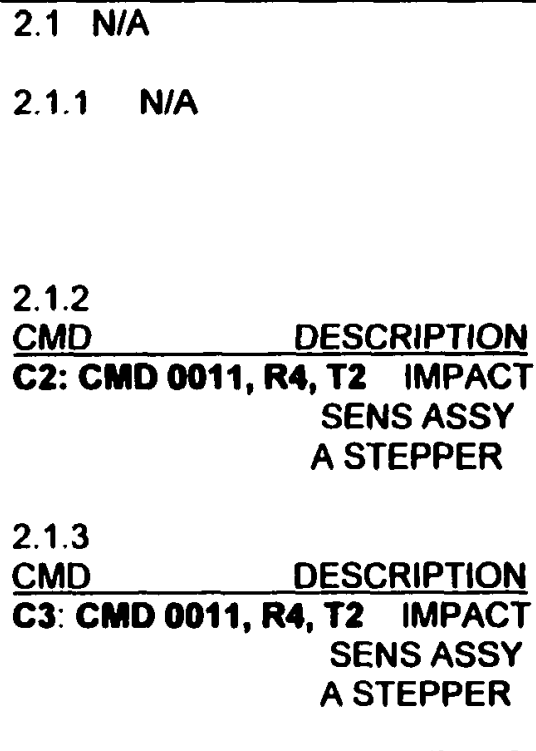 \\
\hline
\end{tabular}




\begin{tabular}{|c|c|c|c|c|}
\hline Type & Description & Crow AssessmentActions & Telemetry Verifiers & Commanding \\
\hline 2 & & $\begin{array}{l}2.2 \text { Disable the pump and assess PLENUM } \\
\text { IAW Checklist B-1. Crew should note the } \\
\text { low plenum pressure readings (PGGA1P } \\
\text { and PGGA2P) of } \sim 48.2 \text { psi. Also, crew } \\
\text { should note that the ENA state is non- } \\
\text { nominal, and that the pressure is low } \\
\text { despite the pump already being enabled. } \\
\text { 2.2.1 Verify PLENUM TLM points. } \\
\text { Plenum does not pump up } \\
\text { However, there is no pressure decrease, ruling } \\
\text { out a hydrazine leak } \\
\text { Command AGE1AB to disable. }\end{array}$ & $\begin{array}{l}2.2 \text { N/A } \\
\\
2.2 .1 \\
\text { SNAP } \\
\text { AGE1AB }=\text { ENA } \\
\text { PGGA1P }=48.2 \\
\text { PGGA2P }=48.2 \\
2.2 .2 \\
\text { SNAP } \\
\text { AGE1AB }=01 S \\
\text { PGGA1P }=48.2 \\
\text { PGGA2P }=48.2\end{array}$ & $\begin{array}{l}2.2 .1 \mathrm{~N} / \mathrm{A} \\
2.2 .2 \\
\text { CMD } \\
\text { C4: } 50463\end{array}$ \\
\hline
\end{tabular}




\begin{tabular}{|c|c|c|c|c|}
\hline Typo & Description & Crew Assessment/Actions & Telemetry Verifiers & Commanding \\
\hline 2 & & $\begin{array}{l}\text { Attempt sequence again to pump } \\
\text { the plenum; recognize there } \\
\text { is not a leak. } \\
\text { Level Select is } 62.50 \text { and gas } \\
\text { generator "A" is disabled } \\
\text { VDE-A GG-A1 ENABLE } \\
\text { Verify plenum pump up; } \\
\text { however, pump does not } \\
\text { appear to work } \\
\text { 2.3.4 VDE-A GG-A1/A2 } \\
\text { DISABLE }\end{array}$ & $\begin{array}{l}\text { 2.3 Verify on SNAP } \\
\text { 2.3.1 AGE1AB = DIS } \\
\text { LVLSEL }=62.50 \\
\text { 2.3.2 AGE1AB = ENA (C5) } \\
\text { 2.3.4 AGE1AB = DIS (C6) }\end{array}$ & 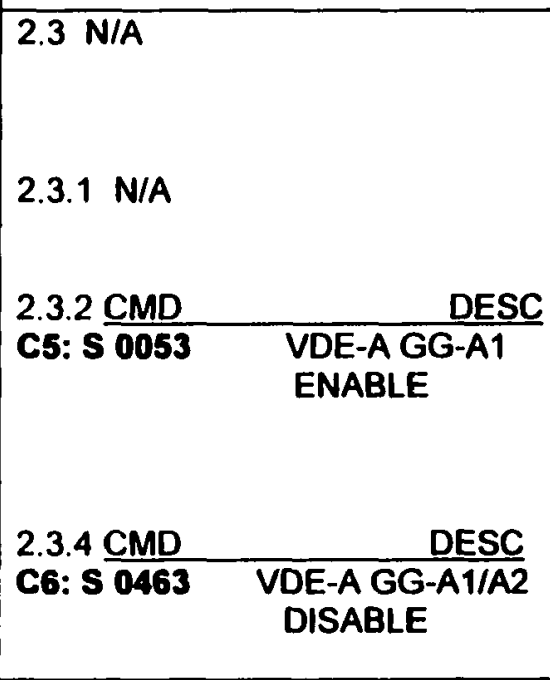 \\
\hline 3 & $\begin{array}{l}\text { 3.1 Troubleshoot and } \\
\text { fix primary transducer: } \\
\text { The primary } \\
\text { transducer is bad. } \\
\text { Page } 7-3 \text { in RW\&B } \\
\text { Technical } \\
\text { Documentation (wiring } \\
\text { diagrams) indicates } \\
\text { that pressure } \\
\text { transducer failures can } \\
\text { be corrected by simply } \\
\text { switching to the } \\
\text { redundant gas } \\
\text { generator transducer. }\end{array}$ & 3.1 View ACSS Screen & $\begin{array}{l}3.1 \\
\text { ACSS } \\
\text { PGGA1P reads } \sim 48.2 \\
\text { PGGA2P reads } \sim 48.2 \\
\text { GGXDCR }=1\end{array}$ & $3.1 \mathrm{~N} / \mathrm{A}$ \\
\hline
\end{tabular}




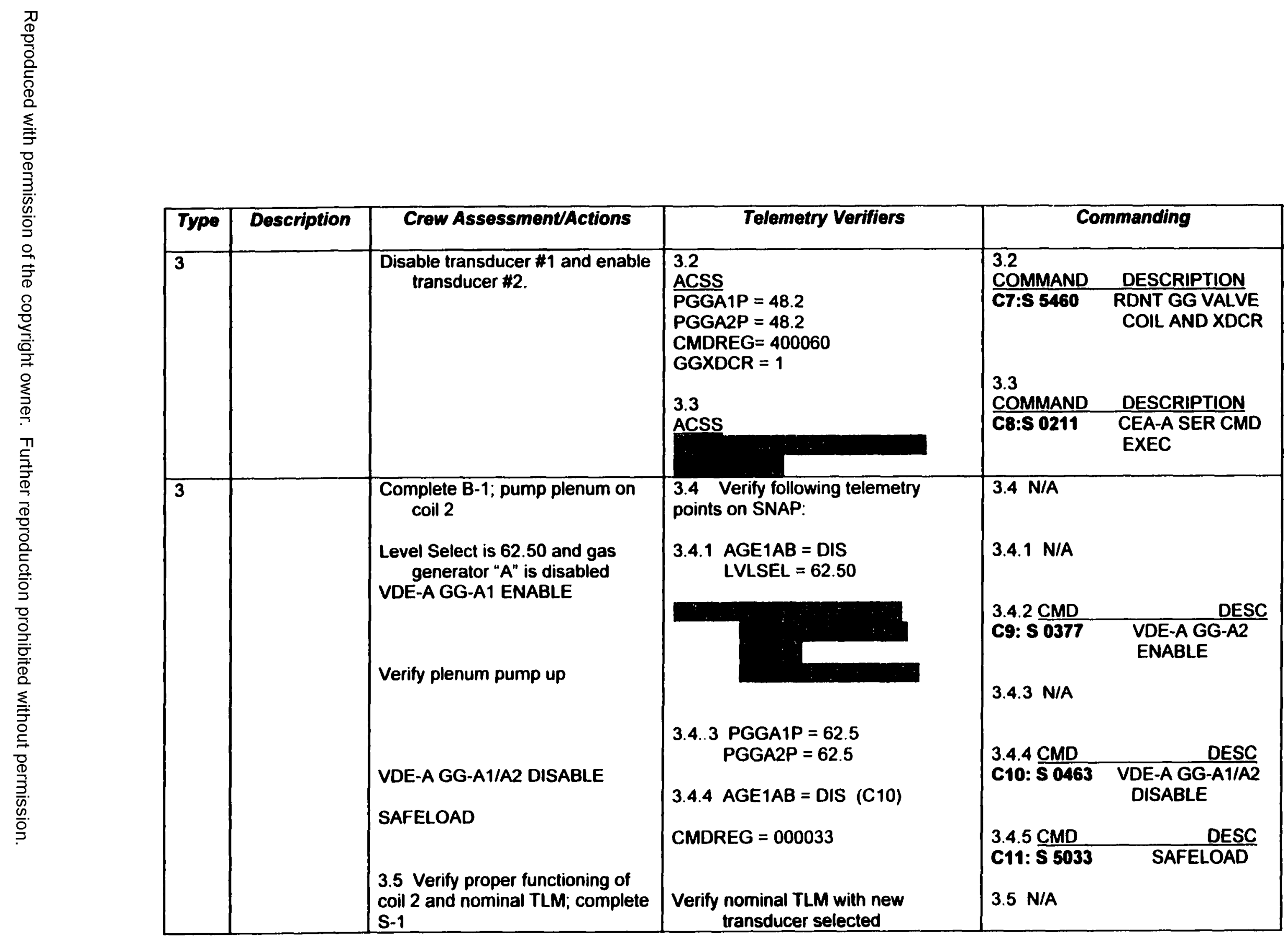

